\title{
Development of Fluorescence Activated Synaptosome Sorting (FASS) and analysis of VGLUT1 synapses from mouse brain
}

\author{
PhD Thesis \\ in partial fulfillment of the requirements \\ for the degree "Doctor rerum naturalium (Dr. rer. nat.)" \\ in the Molecular Biology Program \\ at the Georg August University Göttingen, \\ Faculty of Biology
}

submitted by

Christoph Biesemann

born in

Oelde, Germany 


\section{Declaration:}

I hereby declare that this thesis 'Development of Fluorescence Activated Synaptosome Sorting (FASS) and analysis of VGLUT1 synapses from mouse brain' has been written independently, with no other aids than those indicated.

Christoph Biesemann

September $29^{\text {th }}, 2010$ 


\section{Publications}

Özgür Bayram, Christoph Biesemann, Sven Krappmann, Paul Galland, and Gerhard H. Braus . More Than a Repair Enzyme: Aspergillus nidulans Photolyaselike CryA Is a Regulator of Sexual Development. Mol Biol Cell (2008) vol. 19 (8) pp. 3254-62 


\section{Table of contents}

1.1 Cell types in the brain

1.2 The chemical synapse

1.3 Neurotransmitter systems

$\begin{array}{lll}\text { 1.3.1 } & \text { The glutamatergic system } & 20\end{array}$

1.3.2 The GABAergic system 24

1.3.3 The glycinergic system $\quad 25$

1.3.4 The cholinergic system 26

1.3.5 The dopaminergic system $\quad 27$

1.3.6 The noradrenergic system 28

$\begin{array}{lll}1.3 .7 & \text { The serotonergic system } & 28\end{array}$

1.4 Brain diseases and synaptic dysfunction (Synaptopathies) 29

1.5 Synaptosomes 30

1.6 Proteomics of synaptosomes and synaptosome-derived fractions: Problems with heterogeneity 33

$\begin{array}{lll}\text { 1.6.1 Heterogeneity of the synaptosomal preparation } & 33\end{array}$

$\begin{array}{lll}\text { 1.6.2 Synaptosome proteomics } & 33\end{array}$

$\begin{array}{lll}\text { 1.6.3 Proteomic analyses of synaptic vesicles } & 34\end{array}$

1.6.4 A need for further fractionation of the synaptosomal preparation 35

$\begin{array}{lll}1.7 & \text { Analysis and purification of synaptosome subpopulations } & 36\end{array}$

1.7.1 Immunoisolation of synaptosomes $\quad 36$

$\begin{array}{lll}1.8 & \text { Fluorescence Activated Synaptosome Sorting (FASS) } & 37\end{array}$

$\begin{array}{lll}\text { 1.8.1 Flow analysis and sorting of synaptosomes } & 38\end{array}$

1.8.2 The VGLUT1 ${ }^{\text {VENUS }}$ knock-in mouse as a source of fluorescent VGLUT1 specific synaptosomes for FASS 40

1.8.3 Description of the VGLUT1 ${ }^{\text {VENUS }}$ knock-in mouse model (reproduced with permission of Etienne Herzog)

$\begin{array}{lll}1.9 & \text { Aims of this study } & 46\end{array}$

2 MATERIALS AND METHODS $\quad 48$

$\begin{array}{llr}2.1 & \text { Materials } & \mathbf{4 8}\end{array}$

$\begin{array}{llr}2.1 .1 & \text { Devices } & 48\end{array}$

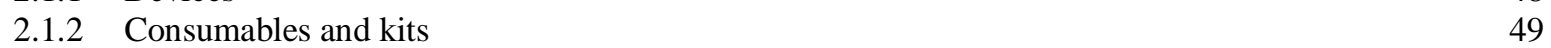


$\begin{array}{llr}2.1 .3 & \text { Software } & 49\end{array}$

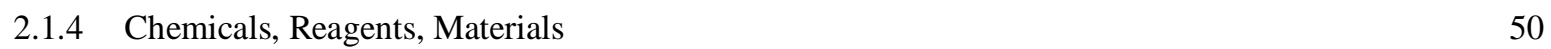

$\begin{array}{ll}2.1 .5 & 51\end{array}$

$\begin{array}{ll}2.1 .6 & \text { Primary antibodies } \\ 2.1 .7 & \text { Secondary antibodies }\end{array}$

$\begin{array}{ll}2.1 .7 & \text { Secondary antibodies }\end{array}$

$\begin{array}{llr}2.2 & \text { Mouse genotyping } & 53\end{array}$

$\begin{array}{llr}\text { 2.2.1 } & 53\end{array}$

$\begin{array}{lll}\text { 2.2.2 } & \text { Agarose gel electrophoresis } & 54\end{array}$

2.3 Subcellular fractionation $\quad \mathbf{5 4}$

2.3.1 Preparation of gradient purified synaptosomes for FASS $\quad 54$

2.3.2 Preparation of synaptic plasma membranes and crude synaptic vesicles 55

2.4 Flow Cytometry and Fluorescence Activated Synaptosome Sorting 56

$\begin{array}{llr}\text { 2.4.1 } & \text { FACS instrumentation } & 56\end{array}$

$\begin{array}{lll}2.4 .2 & \text { Fluorescence activated synaptosome sorting } & 57\end{array}$

$\begin{array}{lll}\text { 2.4.3 Analysis of flow-cytometry data } & 57\end{array}$

$\begin{array}{lll}\text { 2.4.4 Sample recovery after FASS } & 59\end{array}$

2.5 SDS-Polyacrylamide gel electrophoresis $\quad \mathbf{6 0}$

$\begin{array}{llr}\text { 2.5.1 Silver Staining } & 61\end{array}$

$\begin{array}{ll}\text { 2.5.2 Colloidal Coomassie Staining } & 62\end{array}$

$\begin{array}{llr}2.6 & \text { Determination of Protein concentration } & 63\end{array}$

$\begin{array}{llr}2.6 .1 & \text { Bradford assay } & 63\end{array}$

$\begin{array}{lll}2.6 .2 & \text { In gel titration } & 63\end{array}$

$\begin{array}{llr}2.7 & \text { Proteomics } & \mathbf{6 4}\end{array}$

2.7.1 In-gel digestion and mass-spectrometric protein identification 64

2.8 Comparison of proteome and transcriptome data $\quad 65$

2.8.1 Matching of proteins to Gene Symbols $\quad 65$

2.8.2 Analysis, clustering and display of gene expression data as heatmaps 66

$\begin{array}{lll}2.9 & \text { Immuno (Western-)blotting } & 67\end{array}$

$\begin{array}{llr}2.10 & \text { Neuronal Cell culture } & 68\end{array}$

$\begin{array}{lll}2.10 .1 & \text { Rat primary hippocampal neuron culture } & 68\end{array}$

2.10.2 Mouse primary hippocampal neuron culture $\quad 69$

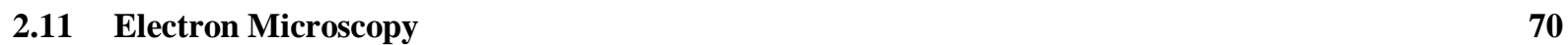

$\begin{array}{lll}2.11 .1 & \text { Electron microscopy of synaptosomes } & 70\end{array}$

$\begin{array}{ll}\text { 2.11.2 Pre-embedding immuno-EM on ultrathin sections } & 71\end{array}$

$\begin{array}{lll}2.12 & \mathbf{I m m u n o f l u o r e s c e n c e ~ l a b e l i n g} \text { and microscopy } & \mathbf{7 1}\end{array}$

$\begin{array}{llr}2.12 .1 & \text { Cultured Neurons } & 71\end{array}$

$\begin{array}{ll}2.12 .2 & \text { Synaptosomes } \\ 2.12 .3 & \text { Bran }\end{array}$

$\begin{array}{ll}2.12 .3 & \text { Brain sections }\end{array}$

$\begin{array}{lll}2.13 & \text { Image analysis using ImageJ } & 73\end{array}$

$\begin{array}{lll}\text { 2.13.1 VGLUT1 } & \text { VENUS } \\ \text { Vynaptosome colocalization assay } & 73\end{array}$

$\begin{array}{lll}3 & \text { RESULTS } & 74\end{array}$

3.1 Use of gradient purified synaptosomes (B) results in faster sorting and higher purity compared to crude synaptosomes (P2)

3.2 Aggregates within the B-fraction can be reduced by passage through the FACSAria and reaggregation can be prevented using Pluronic F-68 
3.3 Sample recovery for Western blotting, electron microscopy and immunofluorescence microscopy after FASS

3.3.1 Vacuum filtration on polycarbonate filters is efficient in recovering synaptosomes for Western blotting and electron microscopy

3.3.2 Centrifugation in custom adaptors allows quantitative recovery of diluted synaptsomes for immunofluorescence microscopy

3.4 FASS in FSC-mode does not remove inhibitory synaptosomes (VIAAT) or myelin (PLP)

3.5 Membrane staining with FM4-64 is compatible with VGLUT1 ${ }^{\mathrm{VENUS}}$ detection and reveals synaptosomes in a lower size range

3.6 Analysis of FASS in FM-mode

3.6.1 FASS in FM-mode enriches VGLUT1 ${ }^{\text {VENUS }}$ synaptosomes as analyzed by flow cytometry 91

3.6.2 Fitting of the flow-data with a multiple normal distribution component model predicts an approximate

8.8-fold relative enrichment of VGLUT1 ${ }^{\mathrm{VENUS}}$ synaptosomes compared to non-fluoescent particles 93

3.6.3 FASS sample protein concentration can be titrated by silver staining and analyzed quantitatively by

Western blotting

3.6.4 FASS co-enriches markers of VGLUT1 synapses and depletes components of microglia, astrocytes and myelin

3.6.5 VAChT is significantly more depleted than VIAAT or VGLUT2 101

3.6.6 FASS reports differential expression patterns of SNAP25/SNAP23, SV2A/B and CPXI/II at excitatory terminals

3.6.7 At least $75 \%$ of FASS-purified VGLUT1 ${ }^{\text {VENUS }}$ synaptosomes have a PSD 103

3.6.8 Analysis of Neuroligin1-4 after FASS reveals that Neuroligin3 and 4 are mainly localized to non-

VGLUT1 synapses

3.6.9 FASS selectively enriches the synaptic pool of glutamate receptors 105

3.6.10 Electron microscopy shows integrity and higher purity of synaptsomes after FASS 106

$\begin{array}{lll}\text { 3.7 A proteomic screening of sorted VGLUT1 } & \text { VENUS } & 107\end{array}$

3.7.1 Proteomics identifies 2044 proteins in sorted VGLUT1 ${ }^{\text {VENUS }}$ and 2333 in unsorted synaptosomes 110

3.7.2 Enrichment and depletion factors of proteins as determined by spectral counting is consistent with results from Western blotting

3.7.3 A comparison with transcriptome data of astrocytes, oligodendrocytes and neurons reveals that FASS of VGLUT1 ${ }^{\mathrm{VENUS}}$ synaptosomes are enriched in neuron-specific and depleted in glia-specific proteins. 118 3.7.4 Clustering according to cell type specific gene expression data provides a global validation for the FASS procedure

3.7.5 Five candidate proteins were chosen on the basis of enrichment in spectral counting, transcriptome comparison and literature research

3.8.1 FXYD6 is expressed throughout the brain and enriches in synaptic subcellular fractions

3.8.2 FXYD6 is neuron specific, localizes to the soma, dendrites and synapses hippocampal neurons 125

3.8.3 FXYD6 localizes to the soma, dendrites and synapses of neurons in situ 127

$3.9 \quad$ TPD52

3.9.1 TPD52 is expressed in many brain regions and localizes to VGLUT1 pre- and postsynapses

3.10 Ly6H

\section{DISCUSSION}

4.1 Methodological developments allow the analysis of FASS samples by independent methods

4.2 FASS in FM-mode enriches intact, single VGLUT1 ${ }^{\text {VENUS }}$ synaptosomes

4.2.1 Evidence from flow cytometry

4.2.2 Evidence from Western blotting and immunofluorescence microscopy

4.2.3 Evidence from electron and immunofluorescence microscopy 
4.3 Differential subcellular localization of several synaptic protein isoforms analysed by FASS

4.3.1 FASS provides evidence that Neuroligin3 and 4 are not present at VGLUT1 synapses

4.3.2 NR2A but not NR2B, is enriched at VGLUT1 synapses

4.4 FASS allows the analysis of a VGLUT1-specific synaptic proteome $\quad 144$

$\begin{array}{lll}\text { 4.4.1 Quantitative analysis by spectral counting } & 144\end{array}$

4.4.2 Transcriptome comparison as a filter for candidate selection 145

$\begin{array}{rrr}4.5 & \text { FXYD6 } & 146\end{array}$

$\begin{array}{lrr}\text { 4.5.1 FXYD6 at VGLUT1 synapses } & 147\end{array}$

$\begin{array}{rrr}4.6 & \text { TPD52 } & 149\end{array}$

$\begin{array}{lll}\text { 4.6.1 TPD52 at VGLUT1 synapses } & 150\end{array}$

$\begin{array}{llr}4.7 & \text { Ly6H } & 150\end{array}$

4.8 Potential improvements and future applications of FASS $\quad 151$

4.8.1 Potential for improvements of the current FASS-method 152

4.8.2 Potential applications of FASS 153

5 APPENDIX 155

$\begin{array}{lr}\text { BIBLIOGRAPHY } & 159\end{array}$ 


\section{Acknowledgments}

First and foremost, I would like to thank Dr. Etienne Herzog for his great supervision and guidance throughout the past four years. I benefited greatly from all the energy and time he invested into supervision and teaching. At the same time, I am thankful to Prof. Dr. Nils Brose for accepting me as a PhD student in his department and for his guidance and support throughout the entire PhD. His input to the project was often critical.

Furthermore I would like to thank:

The members of my PhD committee Dr. Dieter Klopfenstein and Prof. Reinhard Jahn for their support and useful suggestions to the project. The team of the IMPRS MS/PhD Molecular Biology program, Dr. Steffen Burkhardt, Ivana Bacakova and Kerstin Grueniger for creating a supportive environment for us graduate students. The IMPRS Molecular Biology/GGNB and the Graduiertenkolleg 521 for their financial support.

Many more people have contributed to this work and therefore I would like to express my gratitude to:

Sally Wenger for teaching me several laboratory techniques and for her technical contribution to this project. Similarly, I would like to thank Klaus Hellmann, Martin Doerre, Ines Eckhardt, Anja Galinski and Dagmar Michels-Hitzing and the members of the DNA facility Fritz Benseler, Ivonne Thanhäuser and Dayana Schwerdtfeger for their assistance. Dr. Frederique Varoqueaux for helping with the electron microscopy of my samples. Dr. Mads Gronborg for his valuable collaboration in proteomics. Also Uwe Pleßmann for his support regarding the data analysis using the Scaffold software and Dr. Olaf Jahn for his continous advice regarding the proteomic strategy of this project. Dr. Jennifer A. Byrne, Prof. Dr. Reinhard Jahn, Prof. Dr. Käthi Geering and the labs of Prof. Dr. Nave and Dr. Mikael Simons for providing reagents. The staff of the animal facility, especially Rainer Libal, Astrid Ohle, Inga Schauenberg and Tanja Leinert for taking good care of my mice. Markus Krohn, Florian Schütte and Robert Otremba of the fine-mechanics workshop for designing and building several devices essential to the success of this work. My internship student Elodie Kim Grellier who assisted in the characterization of protein candidates and also my collaborators Mattia Aime and Veronique Bernard who characterized FXYD6 by microscopy. Dr. Sven Peter Wichert for enabling the transcriptome data analysis in this work. Simon Bungers for exchanging ideas on ImageJ and for sharing resources in several other aspects our projects. Dr. Liyi Li for his input in the flow cytometry data analysis. 
I was happy to be a member of the department of Molecular Neurobiology over these past years and would like to thank all of my colleagues for the friendly atmosphere and for all of the help and advise that I received from them but did not mention here.

I owe my special thanks to Viki for helping me survive the thesis writing period and for all her support with corrections and editing of the manuscript.

Last but not least, I want to especially thank my parents Arno and Veronika for their never ending encouragement and support that enabled everything for me. 


\section{Abstract}

Signal propagation between neurons in the brain is mediated by chemical synapses. Depending on the neurotransmitters and their receptors, synapses can transmit excitatory, inhibitory, or modulatory signals. A detailed knowledge about the biochemical composition of different subtypes of synapses is an essential requirement in order to study their function in physiology and pathology of the brain.

Synaptosomes are functional nerve-terminals, including pre- and postsynapse, which have routinely been isolated from brain tissue by differential and density gradient centrifugation protocols since 1960. Synaptosome preparations have been utilized widely to analyze the function and protein composition of synapses for several decades. However, the interpretation of corresponding data is confounded by the fact that such synaptosome preparations contain many different types of synaptic particles as well as contaminations by non-synaptic particles from neurons and glia.

In the present study, I established a new method, termed Fluorescence Activated Synaptosome Sorting (FASS), which extends traditional synaptosome preparations by selecting only fluorescent synapses using a cell sorter. A recently established fluorescent VGLUT1 ${ }^{\text {venus }}$ knock-in mouse line was used as a source of fluorescent VGLUT1 synaptosomes. I show here that FASS allows for the isolation of highly pure VGLUT1 synaptosomes containing pre- and post-synaptic elements. Further analysis of FASS purified VGLUT1 $^{\text {venus }}$ synaptosomes provided insights into the synaptic distribution of neuroligins and into the distribution of glutamate receptors to synaptic and extrasynaptic sites. Using proteomic techniques I identified new proteins enriched at VGLUT1 synapses. For 3 of them (FXYD6, Ly6H and TPD52) I confirmed their expression and localization with independent methods.

Taken together, this work shows that FASS is a powerful new tool to purify highly pure synaptic material which can serve to characterize synapses in biochemical, physiological and pathophysiological studies. 


\section{List of Figures}

FIGURE 1: CELL TYPES IN THE CNS

FIGURE 2: SCHEMATIC DRAWING OF A GLUTAMATERGIC CHEMICAL SYNAPSE 18

FIGURE 3: SCHEMATIC REPRESENTATION OF A GLUTAMATERGIC VESICLE 20

FIGURE 4: VGLUT1 AND VGLUT2 MRNA AND PROTEIN EXPRESSION IN THE RAT BRAIN 22

FIGURE 5: SCHEMATIC REPRESENTATION OF EAATS IN THE CEREBELLUM

FIGURE 6: OVERVIEW OF THE PREPARATION AND CONTENTS OF SYNAPTOSOMES AND SYNAPTOSOME-DERIVED FRACTIONS

FIGURE 7 VGLUT1 $1^{\text {VENUS }}$ TARGETING STRATEGY AND PROTEIN EXPRESSION

FIGURE 8: VGLUT1 ${ }^{\text {VENUS }}$ FLUORESCENCE IN THE BRAIN

FIGURE 9: FUNCTIONAL COMPARISION OF VGLUT1 ${ }^{\text {VENUS }}$ AND WILD TYPE-VGLUT1 45

FIGURE 10: FASS OF VGLUT1 ${ }^{\text {VENUS }}$ CRUDE SYNAPTOSOMES (P2) 77

FIGURE 11: FASS ON GRADIENT PURIFIED SYNAPTOSOMES (B) 79

FIGURE 12: AGGREGATE FORMATION IN THE B-FRACTION DURING FASS WAS PREVENTED BY USE OF PLURONIC F-68

FIGURE 13: SAMPLE RECOVERY AFTER FASS

FIGURE 15: FASS IN FSC MODE DOES NOT DEPLETE VIAAT OR PLP 87

FIGURE 17: FM4-64 REVEALS B-FRACTION PARTICLES AT SMALLER SIZE 90

FIGURE 16: FASS IN FM-MODE ENRICHES VGLUT1 ${ }^{\text {VENUS }}$ POSITIVE PARTICLES 93

FIGURE 17: MODELING THE FLOW-DATA AS A MIXTURE OF NORMAL DISTRIBUTIONS 96

FIGURE 20: FLOW CHART OF FINAL FASS PROCEDURE 98

FIGURE 19: ANALYSIS OF SYNAPTIC AND NON-NEURONAL PROTEINS AFTER FASS 99

FIGURE 20: WESTERN BLOT ANALYSIS OF FASS ELUCIDATES THE DIFFERENTIAL
DISTRIBUTION OF PROTEIN ISOFORMS

FIGURE 21: ANALYSIS OF PSD95, NEUROLIGINS AND GLUTAMATE RECEPTORS AFTER FASS 104

FIGURE 22: ELECTRON MICROSCOPY AFTER FASS 107

FIGURE 23 PROTEOMICS SCREEN OVERVIEW 109

FIGURE 24: FOLD CHANGE IN PROTEIN LEVELS BETWEEN SORTED AND UNSORTED SAMPLES AS ASSESSED BY SPECTRAL COUNTING

FIGURE 25: CLUSTERING OF PROTEINS ACCORDING TO THE CORRESPONDING MRNA EXPRESSION IN OLIGODENDROCYTES, ASTROCYTES AND NEURONS 119

FIGURE 26: PROTEIN CANDIDATES SCHEMATIC REPRESENTATION 123

FIGURE 27: FXYD6 IN FASS, SUBCELLULAR FRACTIONS AND DIFFERENT BRAIN REGIONS 125

FIGURE 28: FXYD6 IN PRIMARY HIPPOCAMPAL NEURON CULTURES 126

FIGURE 29: FXYD6 IN SECTIONS

FIGURE 30: TPD52 LOCALISATION AT VGLUT1 SYNAPSES

FIGURE 31: LY6H EXPRESSION AT VGLUT1 SYNAPSES

\section{List of tables}

TABLE 1: COMPONENTS OF THE MAIN, CLASSICAL NEUROTRANSMITTER SYSTEMS 


\section{Abbreviations}

\begin{tabular}{|l|l|}
\hline A & Area \\
\hline AChE & Acetylcholine esterase \\
\hline ACN & Acetonitrile \\
\hline AD & Alzheimer's Disease \\
\hline APS & Ammonium-persulfate \\
\hline bp & Base pair \\
\hline BSA & Bovine serum albumin \\
\hline Cb & Cerebellum \\
\hline ChAT & Choline Acetyltransferase \\
\hline CHT1 & Choline Transporter 1 \\
\hline CID & Collision induced decay \\
\hline CNS & Central nervous system \\
\hline Cp & Caude putamen \\
\hline Ctx & Cerebral cortex \\
\hline Cx & Cerebral cortex \\
\hline Da & Dalton \\
\hline DAT & Dopamine Transporter \\
\hline DBH & Dopamine beta hydroxylase \\
\hline EM & Electron microscopy \\
\hline EPSP & Excitatory Postsynaptic potential \\
\hline ES & Embryonic Stem cell \\
\hline EtOH & Ethanol \\
\hline evt & Event \\
\hline FACS & Fluorescence Activated Cell Sorting \\
\hline FASS & Fluorescence Activated Synaptosome Sorting \\
\hline FC & Fold change \\
\hline FCS & Fetal Calf Serum \\
\hline FSC & Forward Scatter \\
\hline GABA & Gamma-Amino-Butyric Acid \\
\hline GAD & Glutamic acid decarboxylase \\
\hline GFAP & Glial acidic fibrillary protein \\
\hline GFP & Green fluorescent protein \\
\hline GIn & Glutamine \\
\hline Glu & Glutamate \\
\hline GPCR & G-protein coupled receptor \\
\hline h & Hour \\
\hline HBSS & Hank's Balanced Salt Solution \\
\hline HD & Huntington's Disease \\
\hline HQ & High quality \\
\hline HTT & Huntingtin \\
\hline IF & Immunofluorescence microscopy \\
\hline IPSP & Inhibitory Postsynaptic Potential \\
\hline kDa & Kilo Dalton \\
\hline ki & Knock in \\
\hline L & Liter \\
\hline Ly6H & Lymphocyte-antigen 6H \\
\hline m & Meter \\
\hline & \\
\hline
\end{tabular}




\begin{tabular}{|l|l|}
\hline M & Molar \\
\hline MALDI & Matrix Assisted Laser Desorption Ionization \\
\hline NBA & Neurobasal Medium \\
\hline ND & Neutral density \\
\hline NR & NMDA-receptor \\
\hline Ob & Olfactory bulb \\
\hline PAG & Phosphate activated glutaminase \\
\hline PAGE & Polyacrylamide gel electrophoresis \\
\hline PBS & Phosphate Buffered Saline \\
\hline PCR & Polymerase chain reaction \\
\hline PD & Parkinson's Disease \\
\hline PDF & Probability Distribution Function \\
\hline PGT & PBS/Gelatine/Triton-Buffer \\
\hline PLL & Poly-L-Lysine \\
\hline PLP & Myelin Proteolipid protein \\
\hline PSD & Postsynaptic density \\
\hline rpm & Rounds per minute \\
\hline RT & Room temperature \\
\hline S & Second \\
\hline Sc & Spinal cord \\
\hline SDS & Sodium dodecyl sulfate \\
\hline SSC & Side Scatter \\
\hline TBS & Tris-Buffered Saline \\
\hline TEAB & Tetraethylammonium bicarbonate \\
\hline TH & Tyrosine hydroxylase \\
\hline TPD52 & Tumor protein D52 \\
\hline TPH & Tryptophane hydroxylase \\
\hline$U$ & Unit \\
\hline VGLUT & Vesicular Glutamate Transporter \\
\hline VIAAT & Vesicular Inhibitory Amino Acid Transporter \\
\hline VMAT & Vesicular Monoamine Transporter \\
\hline Wt & Wild type \\
\hline X g & g=9.81 ms ${ }^{-1}$ relative centrifugal force \\
\hline & \\
\hline
\end{tabular}




\section{Introduction}

\subsection{Cell types in the brain}

The brain carries out a great variety of very complex tasks. A plethora of information from the body's sensory systems can be processed, integrated and stored by a highly complex network formed by the billions of neurons of the central nervous system (CNS). At the same time the nervous system acts on muscles and glands and is thereby able to control and dynamically adapt behavior and bodily functions.

Neuronal signal processing is based on two modes of signal propagation. Within one neuron, electrical signals travel along the neuronal plasma membranes of dendrites, soma and the axon. Interneuronal communication takes place at points of close contact between neurons. These close contacts have been termed synapses (from Greek

, haptein, to clasp) by Charles Sherrington in 1879. Electrical synapses, which are rare in the vertebrate CNS, transmit signals directly. The vast majority of synapses in the vertebrate CNS are chemical synapses, which transmit information indirectly through neurotransmitter molecules.

While the principal information processing in the brain is mediated by neurons, the most abundant cells in the brain are glial cells, which are essential for proper nerve cell function (Figure 1). Glial cells can be divided into macroglia and microglia. The microglia are considered to be the macrophages of the CNS. In brain development they are important for remodeling neural circuits. Later in life they essentially represent the immune system of the brain and are mediators of inflammatory processes. The macroglia can be further divided into astrocytes and oligodendrocytes.

Before signals are transmitted at the synapse, they are propagated along the axon. Many axons in the CNS are electrically insulated by myelin sheaths. Myelin is formed by oligodendrocytes and allows for fast and energy efficient saltatory signal propagation, while keeping the axonal diameter small. The other type of macroglia, the astrocytes, are the most abundant cell type in the brain. Astrocytes have intimate contact with neurons, capillaries and the cerebrospinal fluid and are responsible for homeostasis of the extracellular environment of neurons. Besides serving trophic functions, their membranes also encapsulate chemical synapses, thus forming the so-called tri-partite synapse. Here, the astrocyte is important for the removal of several neurotransmitters from the synaptic cleft, thereby preventing the spillover of neurotransmitters to neighboring synapses and modulating synaptic transmission. 


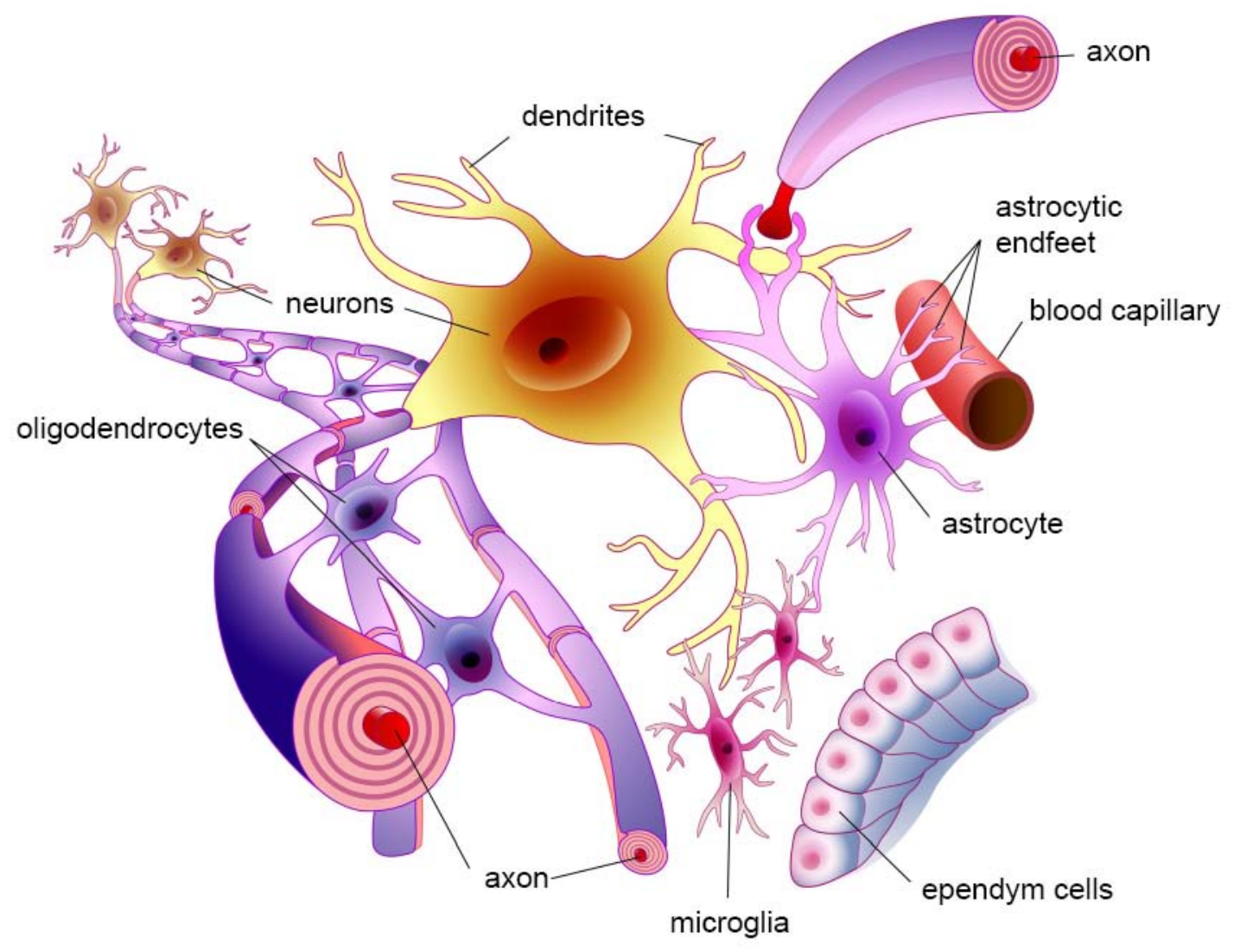

Figure 1: Cell types in the CNS

The brain is a complex structure composed of a variety of different cell types. This figure illustrates the main features and connections of the major cell types in the CNS. Neuronal axons are myelinated by oligodendrocytes. Varicosities of the neuronal axon form synapses on neuronal dendrites. These synapses are encapsulated by astrocytes, which have intimate contacts to both neurons and blood capillaries. Microglia, the immune cells of the CNS are distributed throughout the brain. The ependymal cells line the brain ventricles and the spinal cord canal and produce the cerebrospinal fluid. (Illustration drawn by Jan Ficner, MPI of Experimental Medicine (MPI-EM)) 


\subsection{The chemical synapse}

Upon the arrival of an action potential at the presynapse, fusion competent synaptic vesicles (SVs) release neurotransmitter into the synaptic cleft by means of calciumdependent exocytosis (Fernández-Chacón and Südhof, 1999). In each synapse several tens to hundreds of SVs are generally maintained in different vesicle pools and participate in a cycle that allows them to be reused during prolonged activity (Rizzoli and Betz, 2005; Sudhof, 2004). Active Zones (AZ), which are specialized regions of presynaptic terminals, are the only sites of vesicle fusion and are precisely aligned with the postsynaptic density (PSD). PSDs in turn, are composed of an intricate complex of interlinked proteins containing the neurotransmitter receptors as well as the signaling machinery necessary for receiving the signals sent from the presynapse (Fernández-Chacón and Südhof, 1999). Connected via specific cell adhesion molecules, such as neurexins and neuroligins, AZs and PSDs together form the synaptic signaling complex (Brose, 1999; Fernández-Chacón and Südhof, 1999)

Within the brain, different types of synapses carry out diverse modes of neurotransmission. Excitatory synapses are thought to mainly use glutamate as a neurotransmitter (glutamatergic). Electron microscopy studies have shown that these synapses generally contain round vesicles and electron dense PSDs. Inhibitory synapses are either GABAergic (Gamma-Amino-Butyric-Acid) or glycinergic. In electron microscopy, they generally display unevenly shaped vesicles and much less electron dense material at their PSDs, when using chemical fixatives (Colonnier, 1968; Gray, 1959; Ottersen et al., 1997). Smaller populations of modulatory neurons are generally projecting synapses onto large populations of neurons over entire brain regions in order to convey slow signals, thus tuning the activity of effector neuronal networks. Modulatory systems are signalling mainly through biogenic amines (acetylcholine, dopamine, noradrenaline, serotonin, histamine) and display ultra structural features similar to inhibitory synapses (Gray, 1959). Furthermore, all these synapse types can signal by means of modulatory co-transmitters like zinc, NO and/or neuropeptides. Neuropeptides (and to a certain extent amines) are contained in large dense core vesicles that are present at synapses in lesser amounts as compared to SVs (Barañano et al., 2001; Colonnier, 1968; Gray, 1959). Thus, synapses share many similarities in structure and function, but also display prominent specificities linked to their different roles in neuronal circuitries. 


\section{$1.3 \quad$ Neurotransmitter systems}

As stated above, a variety of neurotransmitters are used by chemical synapses. Synapses can be classified by the neurotransmitter they utilize. But what qualifies a molecule to be a neurotransmitter and what defines which neurotransmitter is used at a given synapse? Classical neurotransmitters meet all of the following four criteria (R. Kandel et al., 2000):

o A neurotransmitter is synthesized in the neuron

$0 \quad$ It is present at the presynaptic terminal and released in amounts sufficient to elicit a specific response in the postsynaptic cell or effector organ

o When administered exogenously it mimics the action of the endogenous transmitter exactly, by activating the same ion channels or second messenger signaling cascades

o A specific mechanism exists to terminate its action or to remove it from the site of action, e.g. the synaptic cleft

These criteria also outline, which components are necessary to define a given neurotransmitter system. The neuron has to contain the substrates and the enzymes necessary to synthesize a given neurotransmitter. The neurotransmitter has to be packaged into vesicles to allow its release by exocytosis. In addition, neurotransmitter specific receptors have to be present on the postsynaptic cell and there have to be enzymes and/or plasma membrane transporters, that remove the neurotransmitter from the synaptic cleft. The enzymes, transporters and receptors of the classical neurotransmitter systems in the vertebrate CNS are listed in Table 1 and further described in the following text.

It should be noted here that the presented work was focused on the glutamatergic system. However, work by others which is described and discussed throughout the text was focused on other neurotransmitter systems. Therefore the glutamatergic system is described in the most detail, but the other neurotransmitter systems are also introduced. 


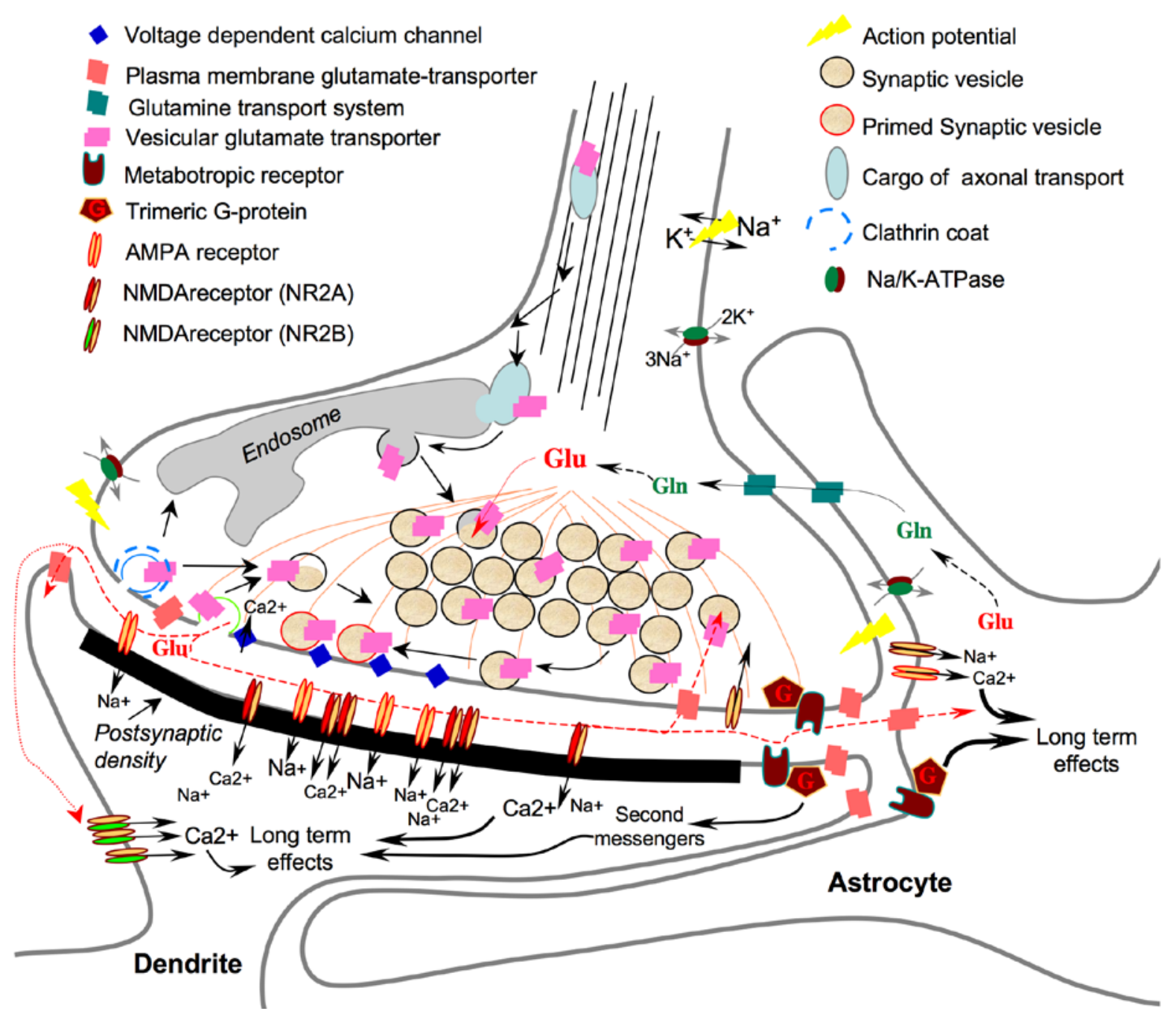

Figure 2: Schematic drawing of a glutamatergic chemical synapse

Several key features and processes characteristic of a generic glutamatergic synapse are illustrated here. Most of these features have correlates in other types of synapses (see Table 1). Synaptic membrane proteins are transported along the microtubules in the axon. Vesicle proteins are sorted/recycled through endosomes, vesicle pools are created and the neurotransmitter glutamate is concentrated into SVs. Action potentials travel along the axon and involve the influx of sodium ions during depolarization and the efflux of potassium during depolarization. The depolarization of the presynaptic terminal leads to influx of calcium ions, through voltage-gated calcium channels. The rise in calcium concentration leads to the exocytosis of vesicles, which were made fusion competent through a process termed priming. After vesicle collapse into the plasma membrane, clathrin mediated endocytosis of SV proteins replenishes the pool of vesicles. The glutamate released into the synaptic cleft binds to a variety of either ionotropic and metabotropic glutamate receptors. The influx of sodium and calcium depolarizes the postsynaptic membrane. Subtypes of ionotropic AMPA and NMDA receptors differ in their ion-permeability and localization. Notably, pharmacological and electrophysiological experiments indicated that NR2B containing NMDA receptors are mostly found in the extrasynaptic compartment of adult neurons (reviewed in (van Zundert et al., 2004)). Glutamate is mainly taken up by astrocytes, which convert it to glutamine before it is shuttled back to the neuron. The excitability of the neuron as well as the plasma membrane transport of glutamate depend on ion-gradients across the cell membrane. To maintain these gradients the $\mathrm{Na} / \mathrm{K}-\mathrm{ATPase}$ driven exchange of $\mathrm{Na}^{+}-$and $\mathrm{K}^{+}-$ ions is of key importance (illustration modified from, (Herzog, 2003)). 


\section{Table 1: Components of the main, classical neurotransmitter systems}

Overview of the main components of the classical neurotransmitter systems. Proteins which can be considered specific, presynaptic markers of the given systems are in bold. PAG: phosphate activated glutaminase, GDH: glutamate dehydrogenase, AAT: aspartate amino transferase, BAT: Branched chain amino acid Amino Transferase, VGLUT: vesicular glutamate transporter, EAAT-excitatory amino acid transporter, GS: glutamine synthetase, GAD-glutamic acid decarboxylase, VIAAT: vesicular inhibitory amino acid transporter, GAT: GABA transporter, GABAT: GABA transaminase, GLYT: glycine transporter, TH: tyrosine hydroxylase, VMAT: vesicular monoamine transporter, DAT: dopamine transporter, MAO: monoamine oxidase COMT: catechol-O-methyltransferase, DBH: dopamine $\beta$ hydroxylase, NET: norepinephrine transporter, TPH: tryptophane hydroxylase, 5-HT: 5-hydroxytrptamine (serotonin), SERT: serotonin transporter, ChAT: choline acetyl transferase, VAChT: vesicular acetylcholine transporter, CHT1: choline transporter, AChE: acetylcholine esterase

\begin{tabular}{|c|c|c|c|c|c|c|}
\hline $\begin{array}{c}\text { Neuro- } \\
\text { transmitter }\end{array}$ & $\begin{array}{l}\text { Biosynthetic } \\
\text { Enzymes }\end{array}$ & $\begin{array}{l}\text { Vesicular } \\
\text { Transporter }\end{array}$ & $\begin{array}{l}\text { Ionotropic } \\
\text { Receptors }\end{array}$ & $\begin{array}{l}\text { Metabotropic } \\
\text { Receptors }\end{array}$ & $\begin{array}{c}\text { Plasma } \\
\text { Membrane } \\
\text { Transporter }\end{array}$ & $\begin{array}{c}\text { Enzymatic } \\
\text { Degradatio } \\
\mathrm{n}\end{array}$ \\
\hline Glutamate & $\begin{array}{l}\text { PAG } \\
\text { GDH } \\
\text { AAT } \\
\text { BAT } \\
\text { GABAT }\end{array}$ & $\begin{array}{l}\text { VGLUT1 } \\
\text { VGLUT2 } \\
\text { VGLUT3 }\end{array}$ & $\begin{array}{l}\text { AMPA: } \\
\text { GluA1 } \\
\text { GluA2 } \\
\text { GluA3 } \\
\text { GluA4 } \\
\text { Kainate: } \\
\text { GluK1 } \\
\text { GluK2 } \\
\text { GluK3 } \\
\text { GluK4 } \\
\text { GluK5 } \\
\text { NMDA: } \\
\text { GluN1 } \\
\text { GluN2A,2B,2C,2D } \\
\text { GluN3A,3B } \\
\text { Orphan: } \\
\text { GluD1 } \\
\text { GluD2 } \\
\end{array}$ & $\begin{array}{c}\text { mGluR: } \\
\text { mGlu1 } \\
\text { mGlu2 } \\
\text { mGlu3 } \\
\text { mGlu4 } \\
\text { mGlu5 } \\
\text { mGlu6 } \\
\text { mGlu7 } \\
\text { mGlu8 }\end{array}$ & $\begin{array}{l}\text { EAAT1 } \\
\text { EAAT2 } \\
\text { EAAT3 } \\
\text { EAAT4 } \\
\text { EAAT5 }\end{array}$ & $\begin{array}{l}\text { GS } \\
\text { AAT } \\
\text { GDH }\end{array}$ \\
\hline GABA & $\begin{array}{l}\text { GAD65 } \\
\text { GAD67 }\end{array}$ & VIAAT & $\begin{array}{l}\text { GABA } A_{A}: \\
\alpha 1, \alpha 2, \alpha 3 \\
\alpha 4, \alpha 5, \alpha 6 \\
\beta 1, \beta 2, \beta 3 \\
\gamma 1, \gamma 2, \gamma 3 \\
\delta \\
\varepsilon \\
\theta \\
\pi \\
\rho 1, \rho 2, \rho 3\end{array}$ & $\begin{array}{c}\mathrm{GABA}_{B}: \\
\text { B } \\
\text { B1 } \\
\text { B2 }\end{array}$ & $\begin{array}{l}\text { GAT1 } \\
\text { GAT2 } \\
\text { GAT3 }\end{array}$ & GABAT \\
\hline Glycine & $\begin{array}{c}\text { none } \\
\text { specific }\end{array}$ & VIAAT & $\begin{array}{l}\text { Glycine: } \\
\alpha 1, \alpha 2, \alpha 3 \\
\beta\end{array}$ & & $\begin{array}{l}\text { GLYT1 } \\
\text { GLYT2 }\end{array}$ & $\begin{array}{c}\text { none } \\
\text { specific }\end{array}$ \\
\hline Dopamine & $\mathrm{TH}$ & VMAT2 & & $\begin{array}{l}\text { Dopamine: } \\
D_{1}, D_{2}, D_{3}, D \\
4, D_{5},\end{array}$ & DAT & $\begin{array}{l}\text { MAO } \\
\text { COMT }\end{array}$ \\
\hline $\begin{array}{l}\text { Noradrena } \\
\text { line }\end{array}$ & DBH & VMAT2 & & $\begin{array}{l}\text { Adrenocepto } \\
\text { rs: } \\
\alpha 1 \mathrm{~A}, \alpha 1 \mathrm{~B}, \alpha 1 \\
\mathrm{D} \\
\alpha 2 \mathrm{~A}, \alpha 2 \mathrm{~B}, \alpha 2 \\
\mathrm{C} \\
\beta 1, \beta 2, \beta 3\end{array}$ & NET & $\begin{array}{l}\text { MAO } \\
\text { COMT }\end{array}$ \\
\hline Serotonin & TPH2 & VMAT2 & $\begin{array}{l}\text { 5- } \mathrm{HT}_{3}: \\
\quad \text { A, B, C, D, E } \\
\text { 5-HT }{ }_{3} \mathrm{AB} \\
5-\mathrm{HT}_{3} \mathrm{~A}\end{array}$ & $\begin{array}{c}5-\mathrm{HT}_{1,2,4-7}: \\
1 \mathrm{~A}, 1 \mathrm{~B}, 1 \mathrm{C}, 1 \mathrm{D} \\
1 \mathrm{e}, 1 \mathrm{~F} \\
2 \mathrm{~A}, 2 \mathrm{~B}, 2 \mathrm{C} \\
4,5 \mathrm{a}, 6,7\end{array}$ & SERT & COMT \\
\hline $\begin{array}{l}\text { Acetylcho } \\
\text { line }\end{array}$ & ChAT & VAChT & $\begin{array}{l}\text { Nicotinic: } \\
\quad \alpha 1, \alpha 2, \alpha 3, \alpha 4, \alpha 5 \\
\alpha 6, \alpha 7, \alpha 9, \alpha 10 \\
\beta 1, \beta 2, \beta 3, \beta 4 \\
\gamma \\
\delta \\
\varepsilon\end{array}$ & $\begin{array}{r}\text { Muscarinic: } \\
\mathrm{M}_{1}, \mathrm{M}_{2}, \mathrm{M}_{3,} \\
\mathrm{M}_{4}, \mathrm{M}_{5}, \mathrm{M}_{6}\end{array}$ & $\begin{array}{c}\text { CHT1 } \\
\text { (choline) }\end{array}$ & AChE \\
\hline
\end{tabular}




\subsubsection{The glutamatergic system}

Glutamate is the most utilized neurotransmitter in excitatory neurotransmission in the CNS, but also functions as an intermediate metabolite, protein building block and energy substrate in neurons and glia (Bak et al., 2006; McKenna, 2007). Nevertheless, neurons are not equipped for de novo synthesis of glutamate from glucose. Instead much of the glutamate used as neurotransmitter is generated by de-amination of glutamine by phosphate-activated glutaminase PAG or by alternative pathways from $\alpha$-ketoglutarate (Bak et al., 2006).

In the presynaptic terminal, glutamate is concentrated into SVs by one of three vesicular glutamate transporters (VGLUT1, -2, -3) (Bellocchio et al., 1998; Gras et al., 2002; Herzog et al., 2001; Herzog et al., 2004a; Herzog et al., 2004b; Takamori et al., 2002; Takamori et al., 2000; Takamori et al., 2001). VGLUTs are the only synapse specific molecular markers of glutamatergic neurotransmission (Takamori, 2006).

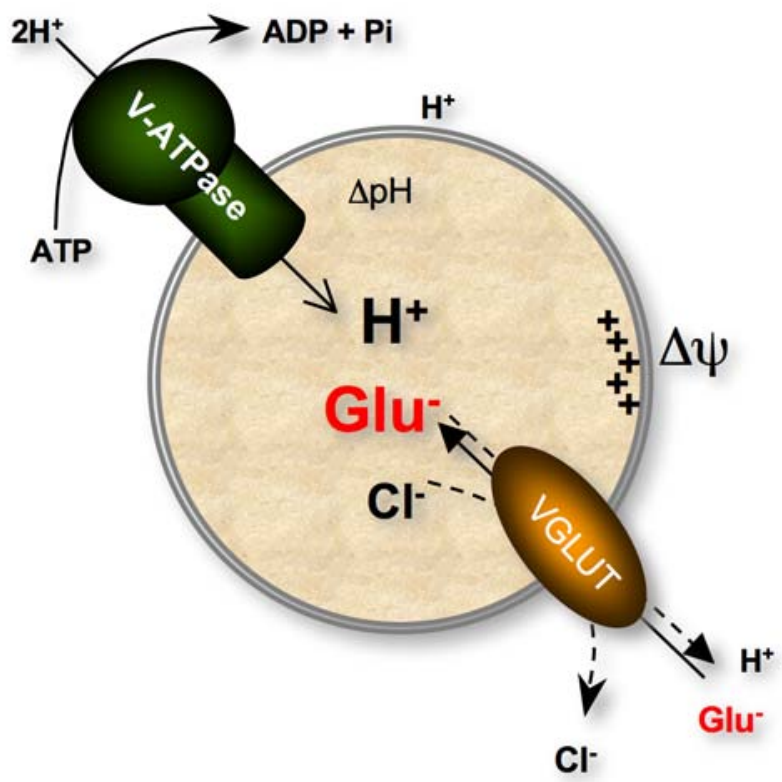

Figure 3: Schematic representation of a glutamatergic vesicle

At glutamatergic synapses the neurotransmitter glutamate is sequestered into SVs by vesicular glutamate transporters (VGLUTs). The energy for this transport mechanism is provided by a vacuolar ATPase, which generates an electrochemical gradient by transporting protons into the vesicle in an ATP dependent fashion. Glutamate transport by VGLUT is mainly dependent on the electrical component of the electrochemical proton gradient, but also depends on chloride. After endocytosis the chloride concentration in vesicular lumen is larger than in the cytosol. Recent evidence suggests that VGLUT1 allows the exit of chloride. A mechanism that is thought to allow for faster loading of the vesicle with glutamate since the efflux of negatively charged chloride enhances the electrical component of the gradient (Schenck et al., 2009) (illustration modified from (Herzog, 2003)) 
The kinetics and properties of glutamate sequestration mediated by the three transporters are virtually identical. However, the differential expression patterns of VGLUT1, 2 , and -3 define three populations (or systems) of glutamatergic neurons that may display differences in their mode of synaptic transmission. System 1 (VGLUT1) is essentially used in cortical regions that arise during post-natal development and that are implicated in cognitive functions and complex motor skill learning. System 2 (VGLUT2) covers mainly regions that develop early and are responsible for sensory functions and autonomic regulatory processes. It was consequently suggested that VGLUT1 could be associated with low release probability terminals and be subject to modulation of synaptic efficacy, whereas VGLUT2 would be restricted to terminals responsible for a more accurate transmission with less modulation of synaptic activity. However, this point remains to be established. System 3 (VGLUT3) exhibits a very discrete distribution that partially overlaps with aminergic (acetylcholine and serotonin) and GABAergic systems and therefore resembles a modulatory system (Bellocchio et al., 2000; Fremeau et al., 2002; Fremeau et al., 2001; Fremeau et al., 2004b; Gras et al., 2008; Gras et al., 2002; Herzog et al., 2001; Herzog et al., 2004a; Schäfer et al., 2002; Takamori et al., 2000; Takamori et al., 2001; Varoqui et al., 2002).

Several research groups have generated and analyzed deletion mutants of VGLUT1, VGLUT2 and VGLUT3. Due to the importance of VGLUTs for glutamatergic synaptic transmission and the wide expression of VGLUT1 and VGLUT2 it was expected that VGLUT1 and VGLUT2 knock-out mice would not survive (Takamori, 2006). Surprisingly, VGLUT1 knock-out mice were born at normal Mendelian ratios and indistinguishable from their littermates for up to two weeks after birth (Fremeau et al., 2004a; Wojcik et al., 2004). Consistent with a role of VGLUT1 in neurotransmitter refilling glutamatergic transmission was abolished or largely reduced in hippocampal slice preparations and cultured autaptic hippocampal neurons (Fremeau et al., 2004a; Wojcik et al., 2004). The residual glutamatergic transmission could be attributed to neurons expressing VGLUT2 birth (Fremeau et al., 2004a; Wojcik et al., 2004). Additionally immunohistochemical studies showed that in many brain regions VGLUT2 is the predominant VGLUT isoform in glutamatergic neurons at early developmental stages (Boulland et al., 2004). VGLUT1 expression was increasing only from two weeks after birth (Boulland et al., 2004). This switch in VGLUT isoform expression coincides with the phenotypic change in VGLUT1 knock-out animals and indicates that VGLUT2 can compensate some of the effects of the VGLUT1 deletion in the first two weeks of life. In contrast to VGLUT1 deletion mutants, newborn VGLUT2 knock-out mice are cyanotic and die (Moechars et al., 2006; WallénMackenzie et al., 2006). VGLUT2 gene expression is high in thalamus, while VGLUT1 gene expression is predominant in hippocampus (Figure 4). Consistent with this expression pattern thalamic neurons cultured from VGLUT2 knock-out mice showed a $95 \%$ reduction in 
glutamatergic synaptic transmission, while hippocampal cultures from the same mice showed no significant difference in glutamatergic transmission (Moechars et al., 2006). In both VGLUT1 and VGLUT2 knock-out mice overall brain morphology and synapse development seems unaltered. However, in both cases reduction in the number of synaptic vesicles and also a change in the synaptic vesicle shape were observed in electron microscopy (Fremeau et al., 2004a; Wallén-Mackenzie et al., 2006).

A
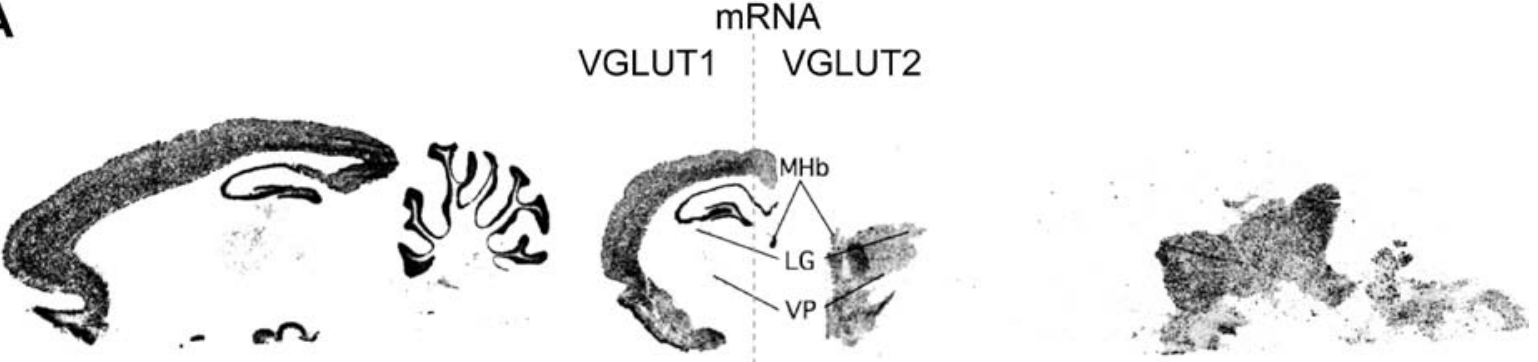

B
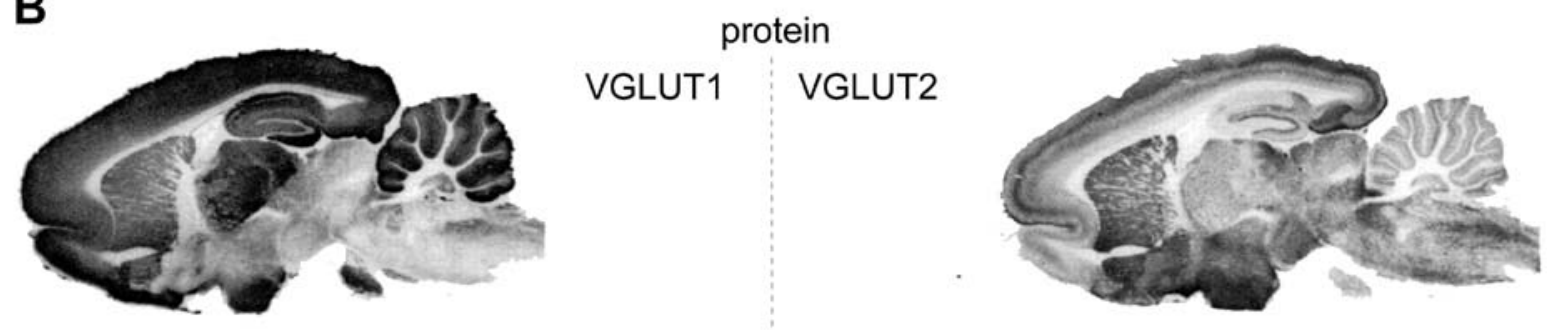

\section{Figure 4: VGLUT1 and VGLUT2 mRNA and protein expression in the rat brain}

VGLUT1 and VGLUT2 show largely complementary expression patterns in the mouse brain. (A) mRNA expression pattern of VGLUT1 (left) and VGLUT2 (right) in the adult rat brain. VGLUT1 is mainly expressed by neurons in higher brain regions, while VGLUT2 is expressed in deeper areas of the brain. (MHb- medial habenular, LG- lateral geniculate thalamic nucleus, VP - ventral posterior thalamic nucleus) (B) Protein expression pattern of VGLUT1 (left) and VGLUT2 (right) in the rat brain. Note the differences in the mRNA to protein expression resulting from the specific localization of the VGLUT proteins to the synapses of neurons projecting to different brain regions. Examples are the thalamo-cortical VGLUT2 projections or the cortico-striatal VGLUT1 projections. (Figure adapted from (Herzog et al., 2001), with permission of the author).

After exocytosis of glutamate filled synaptic vesicles, glutamate acts on postsynaptic plasma membrane glutamate can act as an excitatory neurotransmitter by binding to ionotropic AMPA-, NMDA- or kainate-type glutamate receptors or as modulatory neurotransmitter by binding to metabotropic glutamate receptors (mGluRs) (for review see (Niswender and Conn, 2010; Traynelis et al., 2010). Ionotropic glutamate receptors subunits assemble to form tetramers (Sobolevsky et al., 2009). The AMPA receptors can be homomeric or heteromeric assemblies of the four subunits GluA1-4 and mediate fast 
excitatory transmission. Notably AMPA receptors are impermeable to Calcium when containing the GluA2 subunit. Functional NMDA receptors can be assembled from two GluN1(NR1) subunits together with either two GluN2(NR2) or a combination of GluN2 and GluN3(NR3) subunits. While there is only one isoform of NR1, there are four isoforms of NR2, NR2A-D and two isoforms of NR3, NR3A-B. For proper activation, NMDA-receptors require the simultaneous binding of glutamate and glycine. The levels of glycine in the extraneuronal space is usually sufficient for co-activation, but can be regulated through plasma membrane glycine transporters (see 1.3.3). The glutamate binding site is located on NR2 subunits, while the co-activator can bind to NR1 or NR3 subunits. The conductance of NMDA receptors differs depending on their subunit composition and single channel recordings indicate that cortical neurons contain tri-heteromeric receptors containing NR1, NR2 and NR3 subunits (Sasaki et al., 2002). One prominent feature of NMDA receptors is the voltage dependent blockade of their ion pore by magnesium ions. The block of the pore is only released upon partial depolarization of the membrane. In this way NMDA receptors function as coincidence detectors that sense both postsynaptic depolarization and the release of glutamate. Interestingly different NR2 subunits confer different kinetics to the blocking/unblocking by $\mathrm{Mg}^{2+}$ (reviewed in (Traynelis et al., 2010)).

Central synapses contain both AMPA and NMDA receptors in varying ratios. Therefore, presynaptic glutamate release activates both receptor types. The subunit composition of AMPA and NMDA receptors is developmentally regulated. After birth, NR2B containing NMDA receptors are predominant at immature synapses in the cerebral cortex, hippocampus and in the cerebellum. During synapse maturation a switch occurs after which NR2B containing receptors localize more to extrasynaptic sites, while the mature synapses predominantly contain NR2A containing receptors (for review see (van Zundert et al., 2004)). NMDA receptors localized to extrasynaptic sites, can also be activated by presynaptically released glutamate as a result of spillover and glutamate accumulation during repeated stimulation. The NMDA mediated response to repeated stimulation is essential for processes of synaptic plasticity (Traynelis et al., 2010).

Glutamatergic synaptic transmission is terminated by the removal of glutamate from the synaptic cleft through uptake by astrocytic high-affinity plasma membrane glutamate transporters (EAAT1/GLAST and EAAT2/GLT1). It is thought that within the astrocyte, glutamate is converted to glutamine, which is then shuttled back to neurons to fuel the neuronal glutamate production (Bak et al., 2006; McKenna, 2007). In several cases glutamate can also be taken up by the postsynaptic neurons expressing EAAT3 or EAAT4 (Masson et al., 1999). The distribution of plasma membrane glutamate transporters is especially well illustrated by studies of the glutamatergic synapses formed onto cerebellar purkinje cells (Takayasu et al., 2009) (see Figure 5). There is also evidence for presynaptic 
uptake of glutamate in hippocampal slices (Gundersen et al., 1993). However, none of the known glutamate transporters could be convincingly localized to presynaptic sites and therefore these results still await independent confirmation.

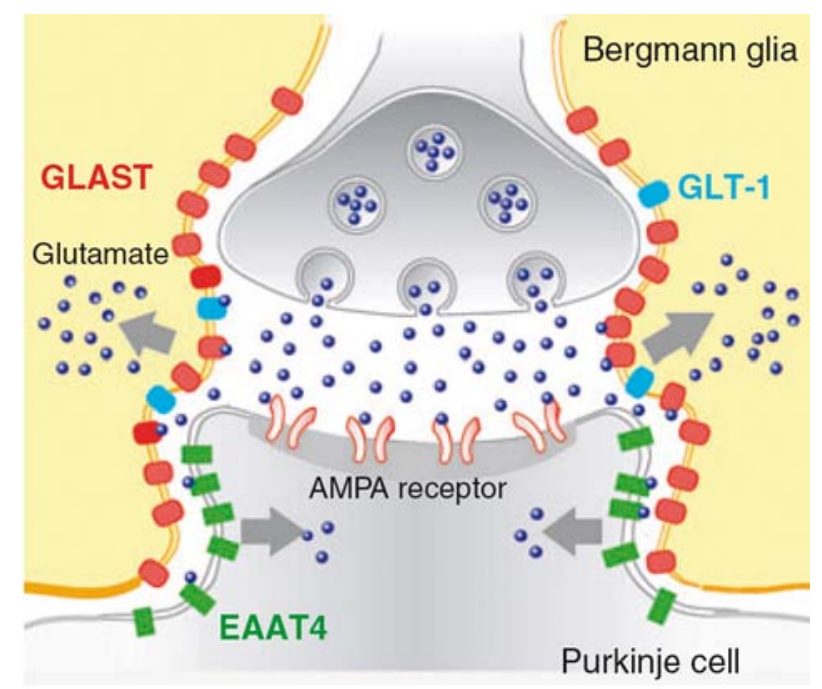

\section{Figure 5: Schematic representation of EAATs in the cerebellum}

This schematic representation shows the distribution of plasma membrane glutamate transporters at excitatory synapses with the example of the glutamatergic synapses onto Purkinje cells in the cerebellum (Figure from (Takayasu et al., 2009)). Note that in the cerebellum GLAST(EAAT1) is the major astrocytic glutamate transporter, while in the forebrain GLT1(EAAT2) is the major isoform (Lehre and Danbolt, 1998).

\subsubsection{The GABAergic system}

Inhibitory synapses can be found in all brain regions and are of great importance for proper nervous system function. They not only suppress irrelevant sensory information but also prevent excessive excitation, which would otherwise lead to epileptic seizures and cell damage. The main inhibitory neurotransmitter in the adult CNS, gamma-aminobutyric acid (GABA) is synthesized by SV associated glutamic-acid decarboxylase (GAD) is loaded into the SVs by the vesicular inhibitory amino-acid transporter (VIAAT, previously also VGAT for Vesicular GABA transporter) (McIntire et al., 1997; Sagné et al., 1997; Wojcik et al., 2006). VIAAT is localized specifically to presynapses and is therefore and excellent marker for inhibitory synapses.

The rate-limiting step in the synthesis of GABA is decarboxylation of glutamate by GAD (for review see (Buddhala et al., 2009)) (Bu et al., 1992). GAD exists in two distinct isoforms, which are referred to as GAD65 and GAD67, according to their respective molecular weight. GAD65 and GAD67 are present as cytosolic homo- and heterodimers (Dirkx et al., 1995; Sheikh and Martin, 1996). GAD65 is more concentrated at nerve terminals, takes part in a multiprotein complex together with VIAAT and can also be directly bound to vesicle membranes (Christgau et al., 1992; Jin et al., 2003). The GABA synthesis 
mediated by GAD65 is important for proper inhibitory neurotransmission (Jin et al., 2003). GAD67 can be bound to vesicles in dimers with GAD65, but is generally spread more evenly throughout the neuron (for review (Martin and Rimvall, 1993)). In a series of papers the Bradford group has reported the specific immunolysis and immunomagnetophoretic isolation of GABAergic synaptosomes using GAD antibodies (Bradford et al., 1989; Docherty et al., 1983; Docherty et al., 1987; Docherty et al., 1985). However, GAD is exclusively cytosolic and should not be accessible to the antibodies. Therefore the specificity of these approaches has been questioned (Whittaker, 1993).

The inhibitory action of GABA is mediated by the postsynaptic GABA receptors. Upon binding to ionotropic $\mathrm{GABA}_{\mathrm{A}}$-receptors, these ligand-gated chloride channels open and the influx of chloride ions into the cell leads to generation of a hyperpolarizing, inhibitory postsynaptic potential (IPSP). In this manner, $\mathrm{GABA}_{\mathrm{A}}$-receptors mediate fast synaptic inhibition. In contrast to this, metabotropic $\mathrm{GABA}_{B}$-receptors are coupled to heterotrimeric $G$ proteins to mediate a slower and more long lasting form of inhibition by gating of potassium channels (Brodal, 2010). The majority of $\mathrm{GABA}_{A}$-receptors are formed by a pentameric assembly of receptor subunits (for review see (Farrant and Kaila, 2007)). The genes encoding $\mathrm{GABA}_{A}$-receptor subunits are grouped into eight families, comprising the $\alpha 1-6, \beta 1$ 3 , y1-3, delta, epsilon, theta, pi and rho1-3 subunits. Even though the majority of receptors is assembled from two $\alpha$, two $\beta$ and one $\gamma$ subunit, the possibilities of combining different subunits results in an enormous diversity of $\mathrm{GABA}_{\mathrm{A}}$-receptors.

\subsubsection{The glycinergic system}

Glycine is the most important inhibitory neurotransmitter in brain stem and spinal cord, where it is involved in motor rhythm generation and in the coordination of spinal reflexes (Hernandes and Troncone, 2009; Legendre, 2001). However, there are also glycinergic synapses in higher brain regions. Besides its role as an inhibitory neurotransmitter glycine is also a co-agonist of NMDA-receptors. Increased levels of glycine correspond to higher NMDA-receptor function.

Serine hydroxymethyltransferase (SHMT), is a highly conserved and ubiquitous enzyme that catalyzes the reversible reaction of serine and tetrahydrofolate to form glycine and 5,10-methylene-tetrahydrofolate. The latter is essential for the synthesis of methionine, pyrimidines and purines. Therefore, this enzyme is important for the nucleotide synthesis in all cells and not specific to glycinergic neurons (Agrawal et al., 2003; Appaji Rao et al., 2003).

The vesicular uptake of glycine is mediated by VIAAT, which is the shared vesicular transporter for glycine and GABA. After release by $\mathrm{Ca}^{2+}$-dependent exocytosis glycine binds to ionotropic glycine receptors. There are four $\alpha$ subunits $(\alpha 1-4)$ and one $\beta$ subunit expressed 
in the CNS. Heteromeric glycine receptors are pentameric assemblies of three/two $\alpha$ and two/three $\beta$ subunits. In the absence of the $\beta$ subunit, $\alpha$-subunits can form homomeric glycine receptors. The subunits show a differential expression pattern in the brain. Since the $\alpha$ subunit contains the glycine binding site it is thought that a subunit expression is a prerequisite for the presence of functional glycine receptors.

At synapses, rapid clearance of glycine from the extracellular space is mediated by plasma membrane glycine transporters (for review see (Betz et al., 2006). Two glycine receptor genes give rise to several splice variants, GlyT1(a, b, c, e, f) and GlyT2(a, b). GlyT1 is widely expressed in astrocytes in all brain regions and was shown to surround glycinergic and non-glycinergic synapses. GlyT2 is mainly expressed in glycinergic neurons of the brainstem and spinal cord. At glycinergic synapses, astrocytic GlyT1 is responsible for the rapid removal of glycine from the synaptic cleft, while the presynaptic GlyT2 is necessary to replenish the pre-synaptic pool of this neurotransmitter (Gomeza et al., 2003; Singer et al., 2009).

As mentioned above, glycine also functions as a NMDA-receptor coagonist at glutamatergic synapses. It has long been thought that the glycine levels at glutamatergic synapses are mainly regulated by astrocytic GlyT1. However, GlyT1 also localizes to glutamatergic postsynaptic densities where it interacts with the NMDA-receptor scaffolding molecule PSD-95 (Cubelos et al., 2005a; Cubelos et al., 2005b). In forebrain-neuron specific conditional GlyT1 knock-out mice the NMDA/AMPA ratio is increased by a factor of 2.15 (Yee et al., 2006). This can be taken as strong evidence for a role of neuronal GlyT1 in the regulation of NMDA-receptors through regulation of glycine levels.

\subsubsection{The cholinergic system}

Acetylcholine is best known for its neurotransmitter function at the neuromuscular junction, a specialized synapse that allows control of muscle contraction. However, acetylcholine is also utilized as a neurotransmitter by a modulatory system of the CNS. Groups of cholinergic neurons are present in several basal forebrain regions and innervate the cerebral cortex, the hippocampus and the amygdala. In addition, cholinergic pontine neurons innervate the brain stem reticular formation and the thalamus (R. Kandel et al., 2000). Cholinergic neurotransmission is important in the regulation of sleep-wake cycles and in regulation of responses to sensory stimuli (R. Kandel et al., 2000).

Production of acetylcholine is mediated by choline acetyltransferase (ChAT), which catalyzes the transfer of an acetyl group from acetyl-CoA to Choline ((Nachmansohn and Machado, 1943). The properties and functions of ChAT in the CNS have been comprehensively reviewed (Oda, 1999)). In electron-microscopic studies ChAT was observed to be intracellularly localized to the cytosol, vesicles and plasma membrane (Lan et 
al., 1995; Martínez-Murillo et al., 1989a; Martínez-Murillo et al., 1989b). ChAT exists two different states at cholinergic synapses, a soluble form (80-90 \%) and a membrane bound form (10-20\%). The nature of the membrane attachment is not clearly established. A Bioinformatic GPI anchor prediction tool ("big PI predictor") fails to identify any potential site for GPI anchoring in the ChAT protein (data not shown), but there is some evidence that ChAT can be attached to the plasma membrane via a GPI-anchor (Smith and Carroll, 1993). Surprisingly, these authors presented evidence that the GPI-anchored ChAT is preferentially intracellular. In summary, ChAT is predominantly cytosolic and only a small fraction of the protein is membrane bound. The nature of the membrane binding and the side of the plasma membrane it is attached to remain controversial.

Acetylcholine present in the cytosol is loaded into SVs by the vesicular acetylcholine transporter. Cholinergic synaptic transmission is mediated by nicotinic and muscarinic acetylcholine receptors (Table 1 ). In contrast to the previously introduced neurotransmitters acetylcholine-mediate neurotransmission is terminated by the degradation of acetylcholine to acetate and choline by the enzyme acetylcholinesterase (AChE). Choline transporter 1 (CHT1) mediates the re-uptake of Choline into the neuron.

\subsubsection{The dopaminergic system}

Dopaminergic neurons in the midbrain provide input to the cerebral cortex and to basal ganglia, where they are involved in reward related learning and behavior, addiction to drugs of abuse and striatal motor functions. Another group of dopaminergic neurons resides in the hypothalamus and is involved in lactation and maternal behaviors (R. Kandel et al., 2000). The loss of dopaminergic neurons of the substantia nigra pars compacta, which normally project to the striatum is the main neurological feature observed in Parkinson's disease (Lotharius and Brundin, 2002). Dopamine is synthesized by tyrosine hydroxylase (Icard-Liepkalns et al., 1993). Packaging in granules or vesicles is performed by the common vesicular monoamine transporter of type 2 in the CNS (VMAT2) and of type 1 elsewhere (VMAT1) (Eiden et al., 2004). This step is critical as dopamine is toxic when left free in the cytoplasm. After release and binding to dopaminergic G-protein coupled receptors (D1-5; for review see (Holmes et al., 2004)), the presynaptic dopamine transporter (DAT) is responsible for the clearance of dopamine from the synaptic cleft (Giros et al., 1996). DAT is a specific marker of dopaminergic neurons and varicosities. 


\subsubsection{The noradrenergic system}

The noradrenergic neurons of the locus coeruleus provide input to the thalamus, cerebral cortex as well as to the brain stem, cerebellum and spinal cord (R. Kandel et al., 2000). In this way noradrenaline affects processes of attention and cognitive functions emotion and sleep regulation. The biosynthesis of noradrenalin depends on the action of dopamine- $\beta$-hydroxylase (DBH). DBH is tightly bound to the to the lumen of SVs, where it converts dopamine to noradrenaline. The dopamine is loaded into SVs by VMAT2. Noradrenalin is released from axonal varicosities, of which only a small proportions form synaptic contacts (Aoki et al., 1998). The effects of noradrenalin in the CNS are mediated by metabotropic adrenoceptors. The adrenoceptor family is composed of $\alpha 1(A, B, D), \alpha 2(A, B, C)$ and $\beta(1,2,3)$, which were grouped according to pharmacological criteria (Bylund et al., 2010). Depending on the isoform they can be expressed on astrocytes, microglia and neurons (Hertz et al., 2004). $\alpha 1$ adrenoceptors are predominantly found on astrocytes, $\alpha 2$ adrenoceptors can be found on pre- and postsynaptic neurons and on astrocytes and $\beta$ adrenoceptors are also found on postsynaptic neurons and astrocytes (Hertz et al., 2004). In fact, the effect of noradrenaline on astrocytes has received much attention because it allows an upregulation of glutamate production during periods of increased excitatory neurotransmission (Hertz et al., 2004). Noradrenaline (also called norepinephrine) can be reimported into the neurons through the norepinephrine transporter (NET), which is not expressed in astrocytes.

\subsubsection{The serotonergic system}

Virtually all regions of the forebrain receive serotonergic input from neurons found in the rostral part of the raphe nuclei. The serotonergic system regulates circadianmechanisms, mood, feeding, thermoregulation and sexual behavior (R. Kandel et al., 2000). The rate limiting reaction in the synthesis of serotonin is the hydroxylation of tryptophane to 5-hydroxytryptophan by tryptophane hydroxylase (TPH). 5-Hydroxytryptophan is then decarboxylated by aromatic acid decarboxylase yielding 5-hydroxytryptamine (serotonin). Of the two TPH isoforms in mammals only TPH2 is expressed by serotonergic neurons in the CNS (Walther and Bader, 2003). The monoamine Serotonin is loaded into SVs by VMAT2 which is the shared vesicular neurotransmitter transporter for dopamine, noradrenaline and serotonin (see 1.3.5 and 1.3.6). Serotonergic neurotransmission is mediated by a large variety of serotonin receptors (5-HT-receptors) which have been divided into seven families $5-\mathrm{HT}_{1}-5 \mathrm{HT}_{7}$ (reviewed in (Bonvento et al., 1994; Staley et al., 1998)). The clearance of serotonin from the synaptic cleft is mediated by the serotonin transporter (SERT) (reviewed 
in (Steiner et al., 2008)). SERT is mainly localized to axonal and presynaptic plasma membrane of serotonergic neurons and to a much lesser extent on astrocytes.

\subsection{Brain diseases and synaptic dysfunction (Synaptopathies)}

Many diseases that affect brain function involve changes of synaptic function, which is not surprising considering the central role of synapses in neuronal communication. The discovery of synaptic dysfunctions that correlate with brain diseases has motivated the use of the term synaptopathy, in order to imply that in these diseases synaptic function is affected (Brose et al., 2010). Considering the complexity of the brain, neurons and synapses it is not always possible to determine whether changes at the level of the synapse are the cause of a given disease or if they result from alterations in more basic neuronal functions. Nevertheless, the list of brain disorders for which synaptic dysfunctions have been described is long and includes Autism, Parkinson's disease, Schizophrenia, Alzheimer's disease and Huntington's disease.

Alzheimer's disease (AD) is the most common form of dementia in the aged population, with 4.6 million new cases each year worldwide (Smith, 2006). AD is a neurodegenerative disease that is characterized by the loss of neurons, synapses as well as the presence of 'senile plaques' containing amyloid- $\beta$ and 'neurofibrillary tangles' containing hyperphosphorylated tau protein (for reviews see (Arendt, 2009; Lee et al., 2001b; Parameshwaran et al., 2008)). Several synaptic proteins have altered expression levels in $A D$. Synaptic dysfunction precedes neuronal loss in the development of $A D$ and seems to affect especially excitatory synapses in cortex and hippocampus (Arendt, 2009). This is underlined by the fact that loss of VGLUT1 and VGLUT2 protein expression levels are correlated with the cognitive decline during AD (Kashani et al., 2007).

Huntington's disease (HD) is a progressive late-onset neurological disorder caused by CAG-repeat expansions in the gene encoding huntingtin (HTT). It is characterized by abnormalities in motor coordination, cognitive impairments and psychiatric manifestations (Group, 1993). The clinical symptoms of perturbed motor coordination coincide with a loss of striatal medium-sized spiny neurons (MSNs) (for review (Cowan and Raymond, 2006)). These inhibitory neurons receive excitatory input from the thalamus and from the cortex and it has been shown that an excess in NMDA-mediated excitation contributes to the loss of MSNs in HD. In HD, MSNs show an increase in surface expression of the NR2B subunit (Fan et al., 2007). Furthermore, the negative effect on the survival of MSNs is mediated by extrasynaptic NR2B containing NMDA receptors, whose downstream signalling cascades affect the neurotoxicity of the mutant HTT in MSNs (Milnerwood et al., 2010; Okamoto et al., 
2009). In contrast, activation of synaptic NMDA-receptor signaling has a neuroprotective effect in MSNs by promoting the formation of HTT inclusions (Okamoto et al., 2009).

Autism spectrum disorders (ASDs) represent a group of neurodevelopmental disorders characterized by atypical social behavior, disrupted verbal communication and unusual patterns of restricted interests and repetitive behaviors with varying severity (Association, 1994). Several cases of hereditary nonsyndromic cases of ASDs have been reported and include individuals with loss-o- function mutations in genes encoding Neuroligin3 and Neuroligin4 (Jamain et al., 2003; Südhof, 2008). Members of the Neuroligin family of cell adhesion molecules are important for proper synapse maturation and function (Varoqueaux et al., 2006). Interestingly, mouse models deficient for Neuroligin3 or Neuroligin4 mimic certain behavioral aspects associated with autistic phenotypes (Jamain et al., 2008; Radyushkin et al., 2009; Tabuchi et al., 2007).

While being far from comprehensive, this short overview of a few prominent cases illustrates that synaptic dysfunction is a hallmark of many brain disorders and that changes in the function, expression levels or subcellular localization of synaptic proteins can be diagnostic or causative for several aspects of these diseases. For this reason the characterization of the protein composition of synapses will be of great importance for a more complete understanding of their role in the physiology and pathology of the brain.

\subsection{Synaptosomes}

Brain tissue homogenization in non-ionic, iso-osmotic media allows the release of synaptosomes, which are resealed nerve terminals separated from the axon and dendrites (for review see (Whittaker, 1993)). The term synaptosome was coined by Victor Whittaker to describe an isolated, functional synaptic particle that contains mitochondria, possesses a transmembrane potential and retains many of the features of pre- and postsynaptic elements, such as SVs, an active zone and a postsynaptic density.

Using a combination of differential centrifugation and sucrose-density gradient centrifugation, synaptosomes were first enriched by Catherine Hebb and Victor Whittaker in 1957 (Hebb and Whittaker, 1958)(see Figure 6 for illustration). Since then, many variations of this subcellular fractionation protocol have been published. Nevertheless, the original sucrose-density gradient procedure yields synaptosomes of the highest purity (Whittaker, 1993). To date, it seems that almost all synapse subtypes, from any brain region, can yield synaptosomes (Whittaker, 1993). Notable exceptions are the large mossy fiber synapses, which might be disrupted under conditions used for preparation of other synaptosomes (Israël and Whittaker, 1965). 
Since their discovery about 50 years ago, a great amount of knowledge about synapse structures and functions has been gained from studies on synaptosomes. For example, some of the first indications that Serotonin and substance $P$ might serve as neurotransmitters in the CNS came from their abundance in synaptosome preparations (CLEUGH et al., 1964; Whittaker, 1959). Many aspects of the plasma membrane uptake of neurotransmitters and their precursors have been studied in synaptosome-derived plasma membranes (Kanner and Schuldiner, 1987). In addition, experiments on synaptosomederived SVs were essential in providing direct evidence that the amino acid neurotransmitters glutamate, GABA and glycine are actively transported and stored in SVs (reviewed in (Maycox et al., 1990)). On September 1, 2010 a pubmed query using the term "Synaptosome" returned 12698 entries. 928 articles contained "Synaptosome" in the title or abstract of which 290 were published between the years 2000 and 2010 . This underlines that the synaptosome preparation was not only instrumental for studies of the molecular function of synapses in the past, but that it remains an important technique to study many aspects of synaptic function. 


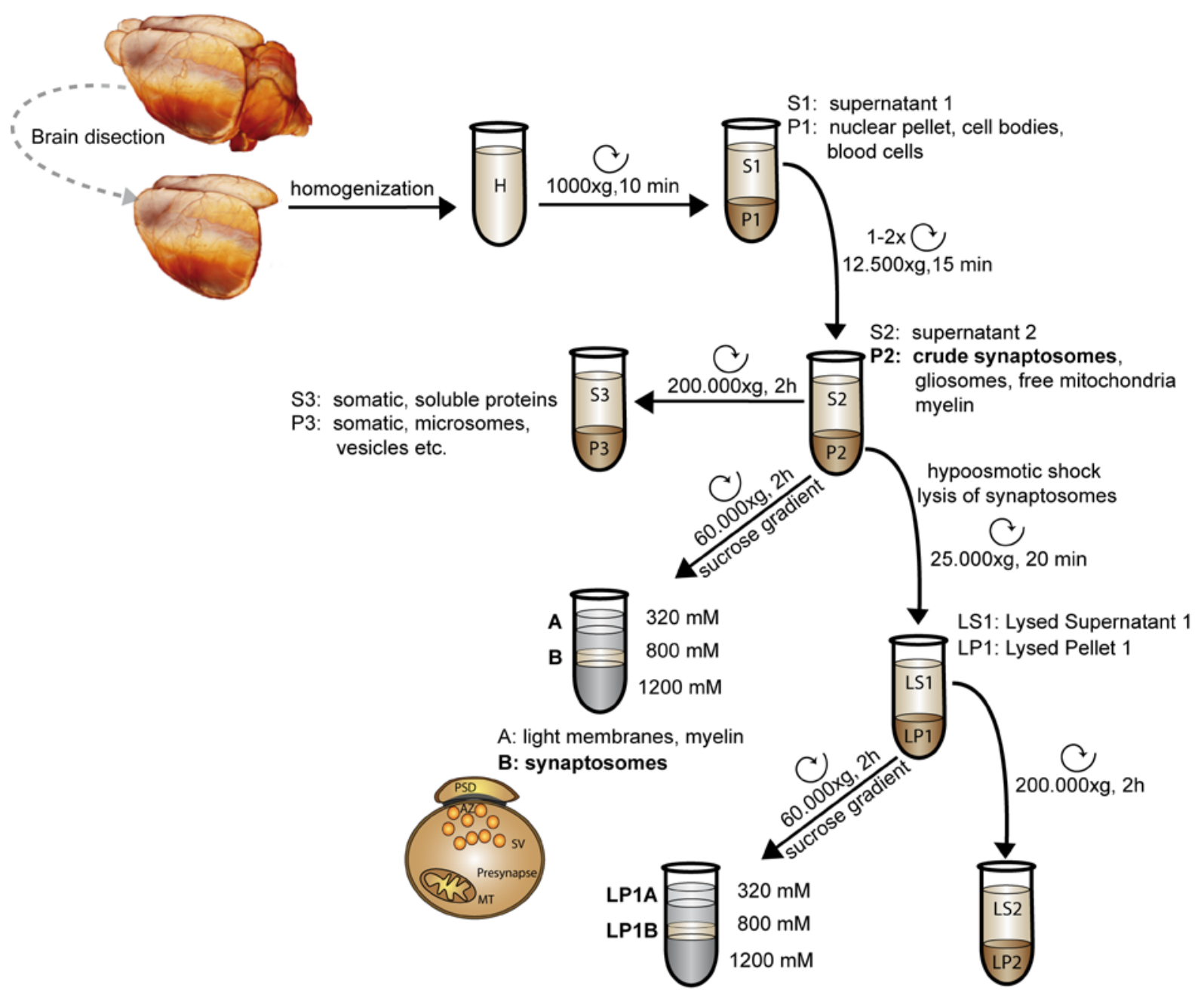

LP1A: light membranes, myelin LP1B: synaptic plasma membrane

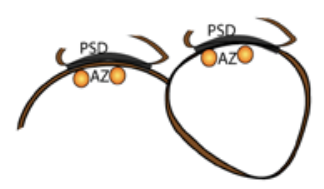

LS2: Lysed Supernatant 2 soluble 'synaptic' proteins LP2: Lysed Pellet 2 crude synaptic vesicles .000
0000000
0000

Figure 6: Overview of the preparation and contents of synaptosomes and synaptosome-derived fractions

This diagram illustrates a subcellular fractionation procedure for the preparation of crude synaptosomes (P2), gradient purified synaptosomes (B), synaptic plasma membranes (LP1B) and crude SVs (LP2). Intact synaptosomes, synaptic plasma membranes and isolated SVs are represented schematically. 


\subsection{Proteomics of synaptosomes and synaptosome-derived fractions: Problems with heterogeneity}

\subsubsection{Heterogeneity of the synaptosomal preparation}

A quantitative electron microscopic study of synaptosomes prepared by the sucrosedensity gradient procedure, reported that $49.1 \%$ of structures visible in the EM were synaptosomes (Dodd et al., 1981). Free mitochondria contributed $2.3 \%$ of particles, while myelin fragments amounted to $3 \%$. The majority of the remaining $46.6 \%$ were unidentified structures, most likely of glial origin (Cotman et al., 1971; Henn et al., 1976). Henn et al. have estimated that about $40 \%$ of gliosomes present in the homogenate are copurified in the synaptosome preparations. Thus the synaptosome fraction is enriched in synaptic particles of different neurotransmitter phenotypes but also contains a large number of non-synaptic particles of neuronal and glial origin. Despite this heterogeneity synaptosomes represent a fraction that is enriched in synaptic particles and therefore allows a much more synapsespecific analysis than the analysis of unfractionated tissue homogenates. For this reason the analysis of the protein content of synaptosomes and synaptosome-derived fractions such as SVs, synaptic plasma membranes and postsynaptic density fractions has lead to an explosion of knowledge about the biochemical makeup of synapses. (for reviews see (AbulHusn and Devi, 2006; Bai and Witzmann, 2007; Bayés and Grant, 2009; Tribl et al., 2006; Tribl et al., 2008).

\subsubsection{Synaptosome proteomics}

The amount of data made available through proteomic analysis of synaptosomes and derived fractions is overwhelming and only few examples can be mentioned here. Schrimpf et al. (2005) utilized the ICAT-approach to further reduce the complexity of the synaptosome proteome. They identified 1131 proteins, 631 of which were identified reproducibly. The identified proteins cover a large spectrum of pre- and postsynaptic proteins, but also include the known glial contaminations. This approach did not identify VGLUTs. On the other hand macroglial contaminations were evident through the identification of GLT-1, MBP, PLP (Schrimpf et al., 2005). Another proteomic study combined 2-DE-MALDI-TOF and a LCMS/MS shotgun approach to catalogue the synaptosomal proteome. Over 900 proteins were identified in this study including many known presynaptic and postsynaptic proteins. A striking feature of this study is that it failed to identify any of the vesicular neurotransmitter transporters. In addition to the identification of proteins, this study also identified several 
posttranslational modifications in synaptosomal proteins, such as glycosylation and acetylation (Witzmann et al., 2005).

Proteomics of synaptosomes has also been applied to study protein expression changes in pathology. A study analyzed changes in synaptsomal protein expression of spinal cord ganglia after spinal nerve injury and proteomics identified 27 proteins to be differentially expressed (Singh et al., 2009). Also a study on the relationship between the administration of antipsychotic drugs used in the treatment of schizophrenia and changes in synaptic protein expression and yielded a total of 17 proteins whose expression levels are differentially regulated as assessed by silver staining after 2-D-gel electrophoresis of synaptosomal proteins (Ji et al., 2009).

The few examples given above illustrate that proteomics of synaptosomes can provide a wealth of information about the protein composition of synaptic fractions and can also be applied to study changes in protein expression in the context of neurological diseases. Even though synaptosomal preparations are enriched in synaptic particles, contaminations by non-synaptic and glial particles also contribute to the proteins identified in proteomic studies of synaptosomes. Furthermore, the synaptosomal preparation contains a mixture of synaptosomes derived from all the different neurotransmitter systems described earlier. Therefore, analysis of synaptosomal proteins reflects the average protein composition of synaptosomal particles from all these neurotransmitter systems. The synaptosomal preparation contains thousands of different proteins, however mass spectrometry based protein identification can not yet fully represent this high complexity and tends to preferentially identify the more abundant proteins within a mixture of proteins (Patterson and Aebersold, 2003). This is exemplified by the fact that the afore mentioned proteomics approaches did not identify the VGLUT proteins and indicates that a further subfractionation of the synaptosomal preparation may be necessary to allow more comprehensive description of it's individual components.

\subsubsection{Proteomic analyses of synaptic vesicles}

One way to further reduce the complexity of the synaptosomal preparation is to break down the synaptosomes into smaller components such as synaptic plasma membranes or SVs. Several investigators have employed these further fractionations in their proteomic studies.

An early, gel based proteomic study on cortical SVs identified 36 vesicle proteins among which were GAPDH, annexin III, $\alpha$-internexin, VDAC1, and Rab14, ZnT3 in cortical SVs. (Coughenour et al., 2004). Later immunoisolation of SV2 containing SVs and synaptic plasma membrane fractions (membranes with docked vesicles - Active zones) and analysis by 2D-16-BAC/SDS PAGE identified 72 SV proteins using MALDI-TOF-MS. (Morciano et al., 
2005). These studies identified several previously known SV proteins and SV associated proteins. Subsequently, a carefully conducted study by Shigeo Takamori in the laboratory of Reinhard Jahn aimed at providing a quantitative description of the lipid and protein composition of average SVs. Following 16-BAC/SDS-PAGE and 1D-SDS PAGE, a total of 410 proteins was identified. These authors also monitored the distribution of 85 proteins among different subcellular fractions by Western blotting, in order to investigate the specificity of these proteins to the SV fraction. Among the SV proteins identified in proteomics were the vesicular neurotransmitter transporters, such as VGLUT1, VGLUT2 and VGAT and the less abundant transporters VAChT and VMAT2 could be identified by Western blotting. The presence of all the different vesicular neurotransmitter transporters illustrates nicely that the SVs isolated here are originating from a variety of different synaptic particles. The authors noted that they observed an unexpected diversity of SNARE and Rab proteins in their isolated SVs. Considering that the diversity of synapses from which synaptic vesicles were isolated this might have reflected the diversity of SVs between different neurotransmitter types. Also the diversity of synaptic, non-synaptic or even non-neuronal particles within the synaptosomal preparation could result in the presence of non-synaptic vesicles in the SV fraction.

These issues were addressed in a later study of the same laboratory. In this study, a quantitative proteomic comparison of immunoisolated VGLUT1 and VIAAT containing SVs based on ITRAQ identified and quantified over 450 proteins (Grønborg et al., 2010). Only 50 proteins were found to be differentially expressed between the two vesicle populations of which only very few were proper SV proteins. These included, ZnT3, SV2A, SV31, which preferentially associated with VGLUT1, and SV2C that was preferentially associated with VIAAT. SV2B was equally distributed between the two vesicle populations. In addition, this study identified a novel VGLUT1-SV protein, called MAL-2. The fact that only few differences could be resolved between SVs of different neurotransmitter systems indicates that the protein correlates of functional specializations of these synapses are not determined by SVs alone, but are probably reflected in the molecular composition of the whole synapse, including cytosolic, vesicular and plasma membrane proteins of the pre-and postsynapse.

\subsubsection{A need for further fractionation of the synaptosomal preparation}

The description above illustrates two key points: (i) The mass-spectrometry based identification of proteins is a powerful tool, which can generate a wealth of data on the protein composition of subcellular fractions in health and disease (ii) Unless quantitative comparative analyses are made it is difficult to differentiate between protein identifications that originate from contaminations and proteins that are specifically enriched in the organelle under investigation. 
As a result of the second point, in the context neurological disease models one can not be certain about the subcellular origin of a phenotype specific change observed by investigations of proteomic differences in the synaptosomal preparation. It would therefore be of great value if it were possible to further purify and sub fractionate the particles contained in the conventional synaptosome preparations. The need for such protocols has already been recognized in the "pre-proteomic era", and in the past several groups have introduced further purification steps, in an attempt to isolate specific subpopulations of synaptosomes at higher purity (reviewed in part by (Whittaker, 1993)).

\subsection{Analysis and purification of synaptosome subpopulations}

As described earlier, the heterogeneity of synaptosome preparations with respect to synaptic and non-synaptic components complicates the analysis of data based on synaptosomes or synaptosome-derived subcellular fraction. If one follows the classification of synapses by neurotransmitter system there are several marker proteins that could be targeted in approaches to isolate neurotransmitter specific subpopulations of synaptosomes (see section 1.3 and bold entries in Table 1). It should be noted here that this separation would still not allow to represent the full diversity of synapses, but has to be considered a first level of classification. Depending on the level of detail required to answer a given scientific question, additional criteria are numerous. Examples would be the anatomical location of the synapse and the corresponding neuron within the CNS, the co-expression of other neurotransmitter-system components, expression of specific components of the release machinery, synaptic cell adhesion molecules or postsynaptic receptors.

Previously published approaches to isolate neurotransmitter specific subpopulations of synaptosomes have employed either immunoisolation of synaptosomes or isolated fluorescently labeled synaptosomes using a cell sorter (FACS).

\subsubsection{Immunoisolation of synaptosomes}

A prerequisite for any successful immunoisolation of subpopulations of intact synaptosomes is the neuron-specific, synaptic surface expression of the targeted marker proteins. In a series of papers the group of Bradford reported the isolation of neurotransmitter system specific subpopulations of synaptosomes on the basis of an immunomagnetophoretic method using antisera raised against several neurotransmitter system specific enzymes ((Bradford et al., 1989; Docherty et al., 1991; Docherty et al., 1987)). The targeted enzymes were glutamic acid decarboxylase (GAD) for GABAergic, Choline acetyltransferase (ChAT) for cholinergic, dopamine- $\beta$-hydroxylase $(\mathrm{DBH})$ for noradrenergic and tryptophane 
hydroxylase (TPH) for serotonergic synaptosomes. Except for $\mathrm{DBH}$, all of the enzymes targeted by the Bradford group are thought to be cytosolic (see 1.3). For this reason the reported enrichments in synaptosome subpopulations are surprising. It has been speculated that the antibodies used had an affinity to plasma membrane carriers or receptors of the same neurotransmitter system, carrying similar epitopes (Whittaker, 1993). The isolated fractions have been studied on the basis of enzyme activity assays, neurotransmitter content and metabolic activity. However, an in-depth analysis of the biochemical composition or morphological characterization of the isolated particles has never been performed. The exact nature of the isolated particles and their purity are therefore very poorly defined. Furthermore, the isolation of neurotransmitter system specific synaptosomes would have been a tremendous advantage for many scientific studies. Nevertheless, no other research group has ever reported the successful application of the immunomagnetophoretic isolation of synaptosomes on the basis of these protocols, which must therefore be regarded as not useful.

\subsection{Fluorescence Activated Synaptosome Sorting (FASS)}

Flow cytometry was originally developed to analyze and sort cells based on DNAcontent and cell-surface markers. Today a large variety of spectrally separable fluorescent dyes can be used to stain various intra- and extracellular structures. Furthermore, a full spectrum of fluorescent proteins has become available, and allows an endogenous labeling of particles for the use in flow applications (Giepmans et al., 2006; Tsien, 2009). A great advantage of flow cytometry is that it analyzes each particle in a sample separately. Flow cytometry can therefore display the true heterogeneity of a sample, without occluding smaller populations due to averaging effects. In addition, improvements of the speed, sensitivity and accuracy of flow-cytometers now allow for very complex, multiparametric analyses and sorting approaches (Herrera et al., 2007). Moreover, for many years biologists have extended the application of flow-analysis and sorting from whole eukaryotic cells to smaller particles, such as bacteria, organelles or even viruses. The organelles analyzed and sorted range from mitochondria and nuclei to vesicles and synaptosomes (Böck et al., 1997). 


\subsubsection{Flow analysis and sorting of synaptosomes}

Flow analysis and sorting of synaptosomes was first reported by Marina E. Wolf and Gregory Kapatos in 1989 (Wolf and Kapatos, 1989a; Wolf and Kapatos, 1989b). The authors analyzed the distribution of several extracellular and intracellular marker proteins by indirect immunofluorescence detection in fixed and permeabilized synaptosomes. The antigens analyzed were a neuron-specific polysialoganglioside recognized by the A2B5 monoclonal antibody (Eisenbarth et al., 1979) to mark synaptosomes, tyrosine hydroxylase (TH) to mark dopaminergic synaptosomes, and glial acidic fibrillary protein (GFAP) to mark astrocytic particles. In addition, the voltage sensitive dye Di-O- $\mathrm{C}_{3}(3)$ was used with unfixed synaptosomal preparations in order to mark particles possessing a membrane potential. In this way, it was reported that $83.9 \%$ of particles are positive for A2B5 and $82 \%$ of all particles posses a membrane potential. In contrast to this, only $7.5 \%$ of particles were positive for GFAP (Wolf and Kapatos, 1989b). The TH positive particles corresponded to $13.1 \%$ of particles in the synaptosome preparation. There was no size preference for the A2B5 or the TH-positive particles. When the $\mathrm{TH}$ positive population was sorted and subsequently analyzed in dot/Western blots it was found that the sorted fraction contained 6fold more $\mathrm{TH}$-immunoreactivity than the starting material. It was also shown that procedures that ablate dopaminergic synapses in the striatum, such as 6-hydroxydopamine lesions, significantly reduce the number of $\mathrm{TH}$ positive particles in the striatal synaptosome preparation (Wolf and Kapatos, 1989a). Following this, Wolf and colleagues used flow cytometry and FACS to analyze the striatal TH positive particles in the context of dopaminergic synapse function and Parkinson's disease (Wolf et al., 1991a; Wolf and Kapatos, 1989c; Wolf et al., 1991b; Wolf et al., 1989).

The studies by Wolf et al pioneered the use of flow analysis and sorting of synaptosomes, but some doubt remains regarding the specificity of their analyses and the purity of the isolated particles. As outlined before, EM studies determined only $50 \%$ of particles in synaptosomal fractions to be actual synaptosomes with a large fraction of unidentifiable particles derived from glia (see 1.6.1). These data are not easily reconcilable with the $82 \%$ synaptosomal content of conventional preparations as reported by Wolf et al. Also the reported value of $7.5 \%$ glial particles appears too low in this respect. While the authors demonstrated that sorting $\mathrm{TH}$ positive particles enriches $\mathrm{TH}$ immunoreactivity, a further biochemical or morphological analysis of the sorted material was not reported. It can be considered as certain that the isolated particles contained intact TH-positive synaptosomes, but it is likely that the particles analyzed and sorted are actually aggregates containing several different types of particles (see Results and Discussion). In this scenario, a single neuronal synaptosome would make the whole aggregate positive, leading to the 
unexpectedly high fraction of synaptosome particles. This would also imply that the isolated TH-positive particles would not be pure. The low fraction of GFAP positive particles however cannot be explained by this, but might be a problem of signal detection or choice of marker. The glial particles contaminating the synaptosome preparations are for the most part also membrane bound (Cotman et al., 1971; Henn et al., 1976). A plasma membrane resident protein of the astrocytes might therefore be more reliable in labeling the astrocyte-derived particles in the synaptosome preparations.

A second research group has also used flow-analysis and sorting in order to study synaptosomes. While Wolf and colleagues studied gradient purified striatal synaptosomes, Gylys et al. analyzed the crude synaptosome fraction (P2) prepared from whole brain (Gylys et al., 2000; Gylys et al., 2004a). The P2 preparation is considerably more contaminated than the gradient purified synaptosomes. Nevertheless Gylys et al. report $62 \%$ of all particles in their P2 preparation to be SNAP25 positive. Using polycarbonate beads as size standards the authors concluded that a region between .75-1.5 micron bead size was highly enriched in intact synaptosomes. This conclusion was based on the observation that $90 \%$ of particles within this size range stained positively for SNAP25, $80 \%$ stained positive for PSD95 and basically all of them were viable as analyzed by calcein-AM staining. In contrast to this, the particles in the smaller size range displayed a more complex distribution, with $70 \%$ viable particles, $40 \%$ of SNAP25 positive and $30 \%$ of PSD-95 positive particles (Gylys et al., 2004a). Based on these observations, Gylys and colleagues treated the particles within this gate as nearly purely synaptic and analyzed them in several studies in the context of Alzheimer's disease (Fein et al., 2008; Gylys et al., 2003; Gylys et al., 2007; Gylys et al., 2004b)

The data by Gylys et al. indicated that particles of all sizes can be SNAP25 positive. Therefore, if synaptosomes were found in a size gate between $.75-1.4 \mu \mathrm{m}$, one has to wonder about the nature of smaller, SNAP25 positive and mostly viable particles, within the P2 preparation. Again, the analyzed particles were not sorted and analyzed for their biochemical composition to allow a determination of the purity of the size-gated population. The authors did however demonstrate that the sorted particles contain round particles of a size between .5 and $1 \mu \mathrm{m}$ by electron microscopy. Unfortunately, only negative staining was employed here to visualize the membrane-bounded particles. Negative staining does not allow to visualize internal vesicles, mitochondria and/or postsynaptic densities, which are commonly used features to identify synaptosomes morphologically.

In summary, the analysis and sorting of synaptosomes from either P2 fraction or gradient purified synaptosomes has been employed by two independent research groups. Even though the biochemical purity of flow-sorted synaptosomes of both groups was not thoroughly analyzed, the 6-fold enrichment in TH-immunoreactivity reported by Wolf et al. is 
encouraging. A several fold enrichment of a given neurotransmitter system specific subpopulation in sufficient amounts to analyze their composition biochemically by Western blotting and proteomics would be of considerable interest to the neuroscience community. However, to date these methods were not applied or further developed to subfractionate synaptosomal particles for proteomic analyses.

\subsubsection{The VGLUT1 ${ }^{\text {VENUS }}$ knock-in mouse as a source of fluorescent VGLUT1 specific synaptosomes for FASS}

Recently, our lab has generated a fluorescent knock-in mouse, which expresses a fusion protein of VGLUT1 and the fluorescent VENUS protein, instead of wild type VGLUT1. These VGLUT1 $1^{\text {VENUS }}$ knock-in mice are indistinguishable from wild type animals in development, morphology and electrophysiology of the VGLUT1 synapses, except that all synapses which normally contain VGLUT1 are now fluorescently labeled by VGLUT1 ${ }^{\text {VENUS }}$ (see below and section 1.8.3) The VGLUT1 ${ }^{\text {VENUS }}$ protein provides a synapse specific endogenous fluorescent label and should therefore enable the flow cytometric detection of unfixed, unpermeabilized and otherwise unstained VGLUT1 ${ }^{\text {VENUS }}$ synaptosomes. The specificity of VGLUT1 (and VGLUT1 ${ }^{\text {VENUS }}$ ) to glutamatergic synapses, together with the bright VGLUT1 ${ }^{\text {VENUS }}$ fluorescence make these mice an ideal model for the development of FASS of glutamatergic synaptosomes. Because of the importance of the VGLUT1 ${ }^{\text {VENUS }}$ knock-in mice to the present work, section 1.8.3 describes the key characteristics of this mouse line. The corresponding unpublished data and text were provided by $\mathrm{EH}$ and modified for the presentation in this thesis.

\subsubsection{Description of the VGLUT1 ${ }^{\text {VENUS }}$ knock-in mouse model (reproduced with permission of Etienne Herzog)}

Before the start of this PhD project, Etienne Herzog $(\mathrm{EH})$ developed the fluorescent VGLUT1 ${ }^{\text {VENUS }}$ knock-in mouse model. The generation and characterization of the VGLUT1 ${ }^{\text {VENUS }}$ knock-in mice has been performed by $\mathrm{EH}$ and collaborators. My own contributions were to the subcellular fractionation experiments, GST-pulldown experiments and tissue preparation at different developmental stages.

\section{GENERATION AND CHARACTERIZATION OF VGLUT1 ${ }^{\text {VENUS }}$ KNOCK-IN MICE}

Homozygous VGLUT1 ${ }^{\text {VENUS }}$ knock-in mice carrying a VENUS CDNA instead of the stop codon in exon 12 of the Vglut1 gene, followed by a neomycin resistance cassette in the 3' untranslated region (vn/vn) were generated by homologous recombination in embryonic stem cells (see Figure 7A). 96 ES cells were initially screened yielding 18 clones with the 
desired homologous recombination identified by Southern blot analysis with a 3' external probe (Figure $7 \mathrm{C}$ ). Several of these clones were injected into the mouse blastocysts to obtain chimeric founder lines that transmitted the mutation through the germline. To eliminate deleterious effects of the neomycin resistance cassette on VGLUT1 ${ }^{\text {VENUS }}$ expression (data not shown), vn/+ mice were crossed with Ella-cre mice, which carry the cre transgene under the control of the adenovirus Ella promoter and express Cre recombinase in early embryonic stages (Lakso et al., 1996). Mice carrying Cre recombined Vglut1 ${ }^{\text {Venus }}$ genes were bred to yield homozygous offspring lacking the neomycin resistance cassette (v/v) (Figure 7). For routine breeding, PCR genotyping was performed (Figure $7 \mathrm{D}$ ). Vglut1 ${ }^{v / v}$ animals are born at the expected Mendelian frequency and are indistinguishable from their $\mathrm{Vglut}^{+/+}$and $\mathrm{Vglut1^{+/v }}$ littermates at birth. v/v mice did not show any obvious behavioral or morphological alterations. Mice had a normal life expectancy, and were fertile even when homozygous v/v animals were crossbred. This is in contrast to VGLUT1 knock-out (KO) mice, which display a severe phenotypic in the third postnatal week and die after around 18 days of life (Wojcik et al., 2004). These observations indicate that the VENUS tag does not interfere with VGLUT1 function in $v / v$ mice.

To assess the proper expression level of VGLUT1 in these mice, native and tagged transporters were systematically quantified from heterozygous and homozygous mouse brain homogenates by Western blotting with infrared fluorescence detection (Figure $7 \mathrm{E}$ ). Venus tagged VGLUT1 migrates with the expected 25-30 kDa shift in SDS-PAGE and displays a slightly more compact migration profile compared to the native protein, which might be due to differences in the resolution of the SDS-PAGE at different molecular weight ranges. Quantifications were performed by comparing normalized integrated intensities of both isoforms in the various genotypes (Figure $7 \mathrm{E}$ ). VGLUT2, which is of comparable molecular weight and displays a similar, diffuse profile and intensity, was used as an internal loading control (not shown; no cross compensations were reported in VGLUT1 knock outs; (Wojcik et al., 2004), (Fremeau et al., 2004a)). No difference between VGLUT1 and VGLUT1 ${ }^{\text {VENUS }}$ expression levels were detected in either genotype. A gene dosage effect could be observed for both isoforms. VGLUT1 $1^{\text {VENUS }}$ therefore replaces VGLUT1 without up- or down-regulation artifacts. To further establish the proper expression of the VENUS mutant we probed Western-blot membranes of v/+ mouse brain subcellular fractionations for VGLUT1 (Figure 7 F). Venus tagged and native isoforms show parallel enrichments to the crude SV fraction (LP2 or SV). When probing with an anti-GFP antibody that recognizes VENUS, we detected a single diffuse band at roughly $92 \mathrm{kDa}$ that also enriches in LP2 fraction. Therefore, VGLUT1 ${ }^{\text {VENUS }}$ seemed to properly target to SVs similarly to the SV marker Synaptophysin. Likewise, brain homogenates prepared from mice at different postnatal developmental 
stages (P1 to P110) and probed with anti-VGLUT1 showed a parallel rise in expression levels of VGLUT1 ${ }^{\text {VENUS }}$ and native VGLUT1 at all time points (Figure $7 \mathrm{G}$ ).

A

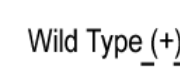

\section{$+1$}

Vector

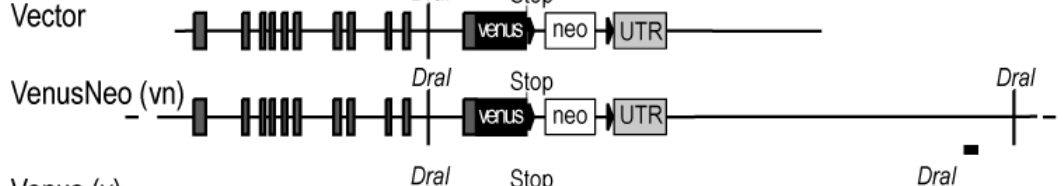

Venus ( $v$ )

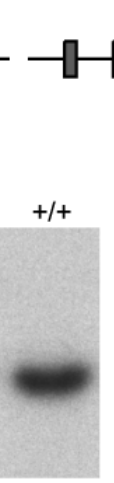

D
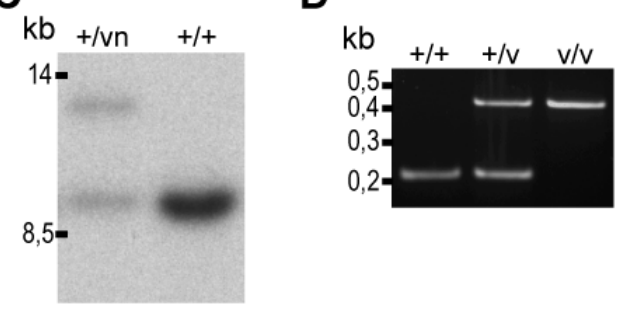

$\mathbf{F}$

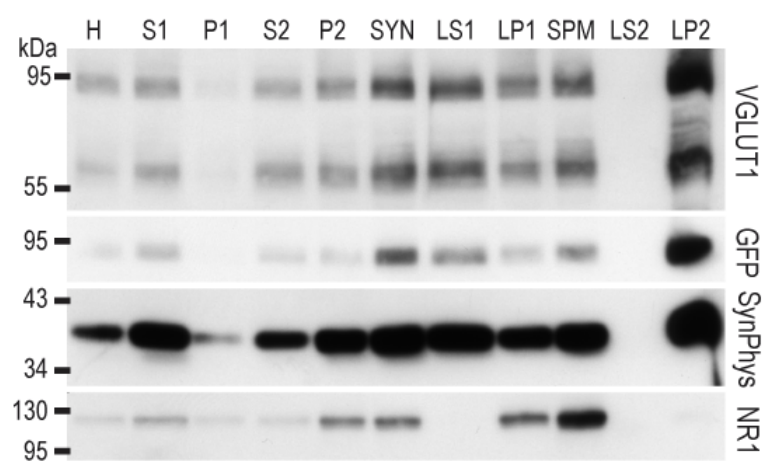

E

G
B

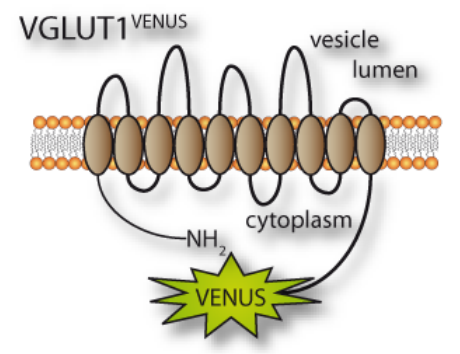

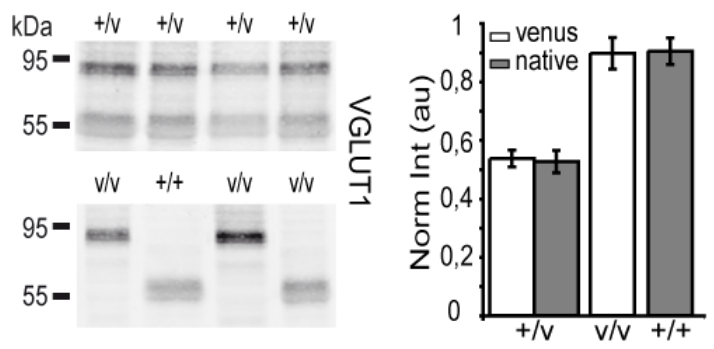

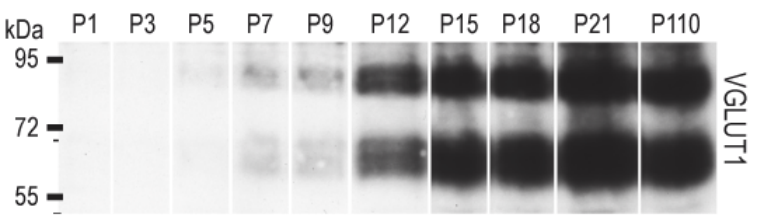

\section{Figure 7 VGLUT1 ${ }^{\text {VENUS }}$ targeting strategy and protein expression}

(A) VGLUT1 ${ }^{\text {VENUS }}$ targeting strategy showing the wild type VGLUT1 gene locus, the VGLUT1 $1^{\text {VENUS }}$ targeting vector, the recombined locus after recombination in ES-cells and the final version of the VGLUT1 ${ }^{\text {VENUS }}$ gene locus after Cre-mediated excision of the neomycin resistance cassette by breeding with a Cre-expressing mouse-line. The position of Dral restriction sites and the probe for Southern blot is indicated. (B) Topology of the VGLUT1 $1^{\text {VENUS }}$ protein in the SV membrane, Venus is attached at the cytoplasm c-terminus of the protein. (C) Successful recombination in ES-cells was demonstrated by Southern blotting. The knock-in allele is detected by the larger size of the Dral restriction fragment recognized by the probe indicated in (A). (D) Genotyping PCR showing the presence of wild type and knock-in alleles in wild type $(+/+)$, heterozygote $(+/ \mathrm{V})$ and homozygote $(\mathrm{v} / \mathrm{V})$ VGLUT1 ${ }^{\text {VENUS }}$ knock-in mice. (E) Example blots of quantitative Western blot analyses of VGLUT1 and VGLUT1 ${ }^{\text {VENUS }}$ protein expression in wild type, heterozygous and homozygous knock-in animals. (F) Subcellular fractionation experiment showing the distribution of VGLUT1 and VGLUT1 ${ }^{\text {VENUS }}$ in comparison to classical SV marker Synaptophysin (SynPhys) and the postsynaptic NMDA-type glutamate receptor subunit 1 (NR1/GluN1). Note that the Venus moiety in VGLUT1 ${ }^{\text {VENUS }}$ can also be recognized using GFP antibodies (see Figure 6). (H: Homogenate, S1/2: Supernatant1/2, P1: nuclear pellet, P2: crude synaptosomes, SYN: 
intact gradient purified synaptosomes (B), LS1: lysed supernatant 1, LP1: lysed P2 pellet1, SPM: gradient purified synaptic plasma membranes from lysed P2 (LP1B), LS2: lysed P2 supernatant 2, LP2: crude SV fraction) (G) Western blot of VGLUT1 and VGLUT1 VENUS protein expression in heterozygous mice between postnatal day 1 (P1) and postnatal day 110 (P110).

LOCALIZATION OF VENUS FLUORESCENCE IN VGLUT1 ${ }^{\text {VENUS }}$ MOUSE CENTRAL NERVOUS SYSTEM.

Paraformaldehyde fixed brains of VGLUT1 ${ }^{\text {VENUS }}$ mice display a bright and distinct fluorescence signal excited optimally at $514 \mathrm{~nm}$ and collected in a 520-560 nm range. Similar excitation and collection leads to a barely detectable background autofluorescence in $+/+$ littermates (Figure $8 \mathrm{AB}$ ). In sagittal brain sections, VGLUT1 ${ }^{\text {venus }}$ fluorescence is prominent in olfactory bulbs, cortex, striatum, hippocampus, thalamus and cerebellar cortex (Figure $8 A B$ ). All these regions are known to contain dense VGLUT1 innervations. Higher magnifications show typical VGLUT1 neuropil staining patterns in hippocampus, cortex and cerebellum. As expected, no VENUS signal is detected in the cell body layers (Figure $8 \mathrm{CDE}$; see (Bellocchio et al., 1998; Herzog et al., 2001; Kaneko et al., 2002). Further colocalization studies showed a proper association of VGLUT1 ${ }^{\text {venus }}$ with presynaptic markers and an apposition to typical glutamatergic postsynaptic markers, while no aberrant overlap is seen with VGLUT2, VGLUT3 or VIAAT (data not shown). Together, these morphological observations demonstrate that the VENUS tag does not interfere with the spatiotemporal expression pattern and subcellular localization of VGLUT1 ${ }^{\text {VENUS }}$. It is properly targeted to excitatory presynaptic terminals and its distribution in brain is indistinguishable from that of VGLUT1. 


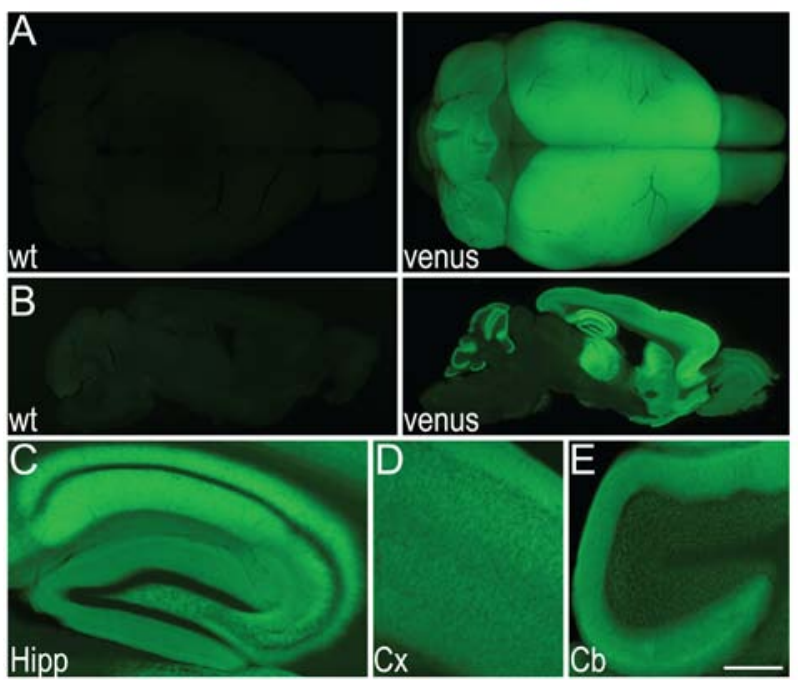

Figure 8: VGLUT1 ${ }^{\text {VENUS }}$ fluorescence in the brain

(A) Whole brain imaged on a fluorescence binocular equipped with a YFP-filter set, showing the bright VGLUT1 $1^{\text {VENUS }}$ fluorescence signal (right) in comparison to the wild type brain (left). (B) Overview of a sagittal section of the brain of a wild type (left) and VGLUT1 ${ }^{\text {VENUS }}$ knock-in mouse. (C,D,E) Higher magnification images of VGLUT1 ${ }^{\text {VENUS }}$ fluorescence in the hippocampus (C, HIpp), cerebral cortex $(D, C x)$ and cerebellum $(E, C b)$ of VGLUT1 ${ }^{\text {VENUS }}$ knock-in mice (scale bar $A-B=2 \mathrm{~mm}, C-D=500 \mu \mathrm{m}$ ).

\section{FUNCTIONAL CHARACTERIZATION OF VGLUT1 ${ }^{\text {VENUS }}$}

After having verified the proper expression, targeting and fluorescence of the tagged transporter, we examined the functional properties of VGLUT1 ${ }^{\text {VENUS }}$. Prior to the establishment of the knock-in mouse line, we used VGLUT1 ${ }^{\text {VENUS }}$ expressing Semliki Forest viruses to successfully rescue the phenotypic defects of VGLUT1-/- neurons (Wojcik et al., 2004). We then used this cDNA construct to generate stable BON cells expressing VGLUT1 ${ }^{\text {VENUS }}$ and checked for vesicular uptake properties. VGLUT1 ${ }^{\text {VENUS }}$ loads similar amounts of glutamate into BON vesicles as compared to native VGLUT1 in the same experiment (data not shown). We then took $\mathrm{v} / \mathrm{v}$ and $+/+$ littermate newborns to generate hippocampal autaptic neuron cultures and performed the electrophysiological characterization of glutamatergic transmission as it had earlier been performed on VGLUT1/- mice (Wojcik et al., 2004) (Figure 9). Our electrophysiological data showed that the transmitter release characteristics of v/v neurons are identical to those of $+/+$ cells (Figure 9 ). We previously characterized the interaction of VGLUT1 with EndophilinA1 via a C-terminal proline rich motif of VGLUT1 (Vinatier et al., 2006). This interaction was shown to influence the recycling of VGLUT1 positive SVs (Voglmaier et al., 2006). We performed a GST pulldown experiment to compare the ability of native and VENUS tagged VGLUT1 to interact with GST-endopilinA1 fusions (Figure $9 \mathrm{G}$ ). Similar to native VGLUT1, VGLUT1 ${ }^{\text {VENUS }}$ bound to EndophilinA1 in an SH3-domain dependent manner. Altogether, these data show that VGLUT1 $1^{\text {VENUS }}$ is a fully functional fluorescent variant of VGLUT1. 
A

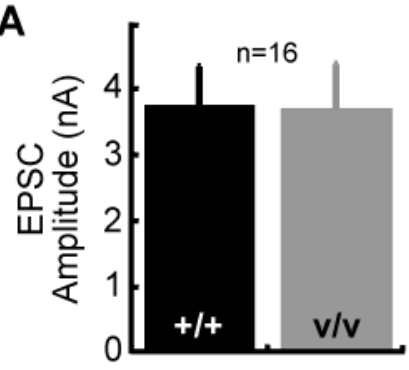

C

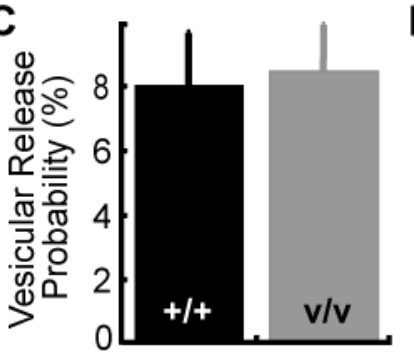

B

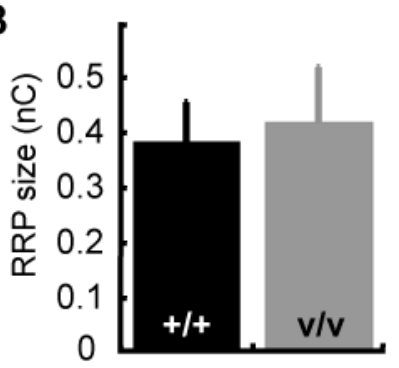

D
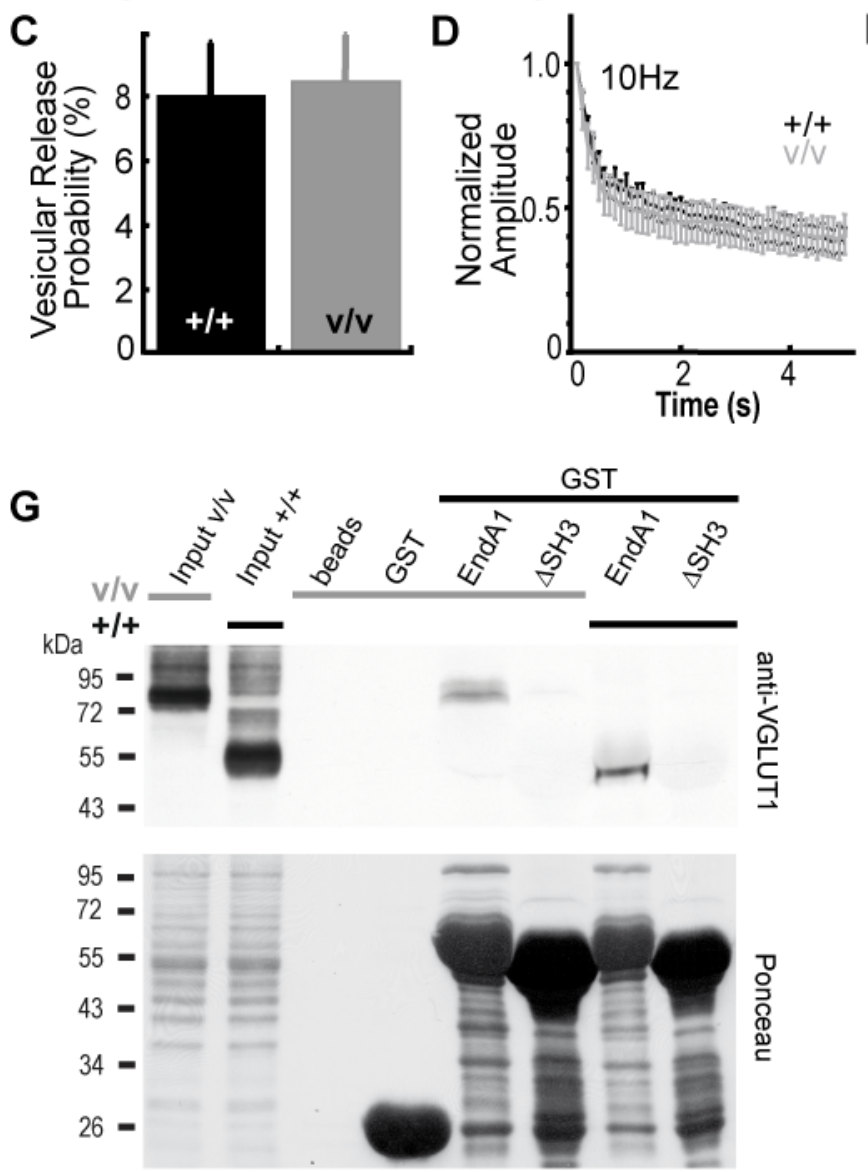

E

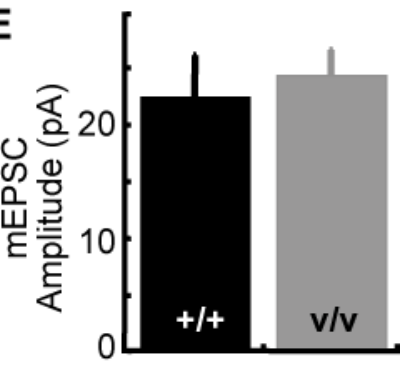

F

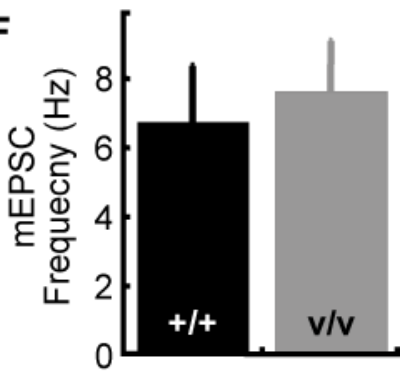

\section{Figure 9: Functional comparison of VGLUT1 ${ }^{\text {VENUS }}$ and wild type-VGLUT1}

(A-F) Electrophysiological characterization of autaptic hippocampal neurons from VGLUT1 $1^{\text {VENUS }}$ and wild type mice. Note that VGLUT1 ${ }^{\text {VENUS }}$ knock-in neurons show unaltered EPSC-Amplitude (A), readible-releaseble pool (RRP) -size (B), vesicular release probability (C), synaptic depression during $10 \mathrm{~Hz}$ stimulus (D) and miniature EPSC amplitude (E) and frequency $(F)$. (G) Full length Endophilin pulls down VGLUT1 ${ }^{\text {VENUS }}$ and VGLUT1 from lysates of wild type and VGLUT1 ${ }^{\text {VENUS }}$ P2 fractions (input lanes). The pulldown experiments were performed using either empty beads, GST alone, GST fused to EndophilinA1 (EndA1) or GST fused to a SH3-domain deletion mutant of EndophilinA1 $(\triangle S H 3)$ (see Ponceau). The SH3-domain of EndophilinA1 had previously been shown to mediate the interaction of EndophilinA1 with VGLUT1. Note that no VGLUT1 signal is detected when the $\triangle \mathrm{SH} 3$ mutant of EndophilinA1 is used in the experiment. 


\subsection{Aims of this study}

As outlined in the previous paragraphs, the brain is highly complex and composed of many different types of synapses that are surrounded by and connected to non-synaptic components of neurons and glia. Synaptosomes have been used extensively to characterize the biochemical composition and functional characteristics of synapses in physiology and pathology (Whittaker, 1993). However, the heterogeneity of the synaptosome preparation makes it difficult to interpret synaptosome-derived data in many contexts. Studies on immunoisolated neurotransmitter system specific synaptic vesicles have revealed little difference in their biochemical composition. Therefore, functional differences, which exist between synapses, cannot be attributed to differences in these organelles alone, but are probably reflected in the full protein complement of the synapse. Our knowledge about the differences in the protein composition of different synaptic subtypes is growing steadily, but is still limited. The availability of protocols for the isolation of specific synaptosome subpopulations at high purity would be of great value for the biochemical analysis of subpopulations of synapses in heath and disease. Several groups have employed strategies to isolate subpopulations of synaptosomes using either immunoaffinity or flow-sorting methods (see $1.7 ; 1.8$ ). However, the approaches published to date are either limited by low sample yield, by the availability of specific marker molecules, and by the methods used to characterize the isolated subpopulations of synaptosomes at the biochemical level. Due to the lack of specific and accessible markers, the glutamatergic population of synaptosomes could not be targeted for selective purification by any group before. The discovery of the VGLUT proteins provided the first specific markers for glutamatergic synapses. Due to the intracellular location of the VGLUT proteins, immunoisolation protocols for intact synaptosomes by targeting VGLUTs are not feasible. The development of the VGLUT1 ${ }^{\text {VENUS }}$ knock-in mouse line provided an interesting model, which is ideally suited for the application of FASS for the purification of the VGLUT1 specific subpopulation of synaptosomes.

Therefore the aims of the presented study were:

o The establishment of methods to allow for Fluorescent Activated Synaptosome Sorting (FASS) of VGLUT1 ${ }^{\text {VENUS }}$ synaptosomes in sufficient amounts for biochemical analyses.

o The analysis of sorted VGLUT1 $1^{\text {VENUS }}$ synaptosomes at the biochemical and microscopic level.

o The screening for and basic characterization of proteins not previously known to localize to or function at VGLUT1 synapses 


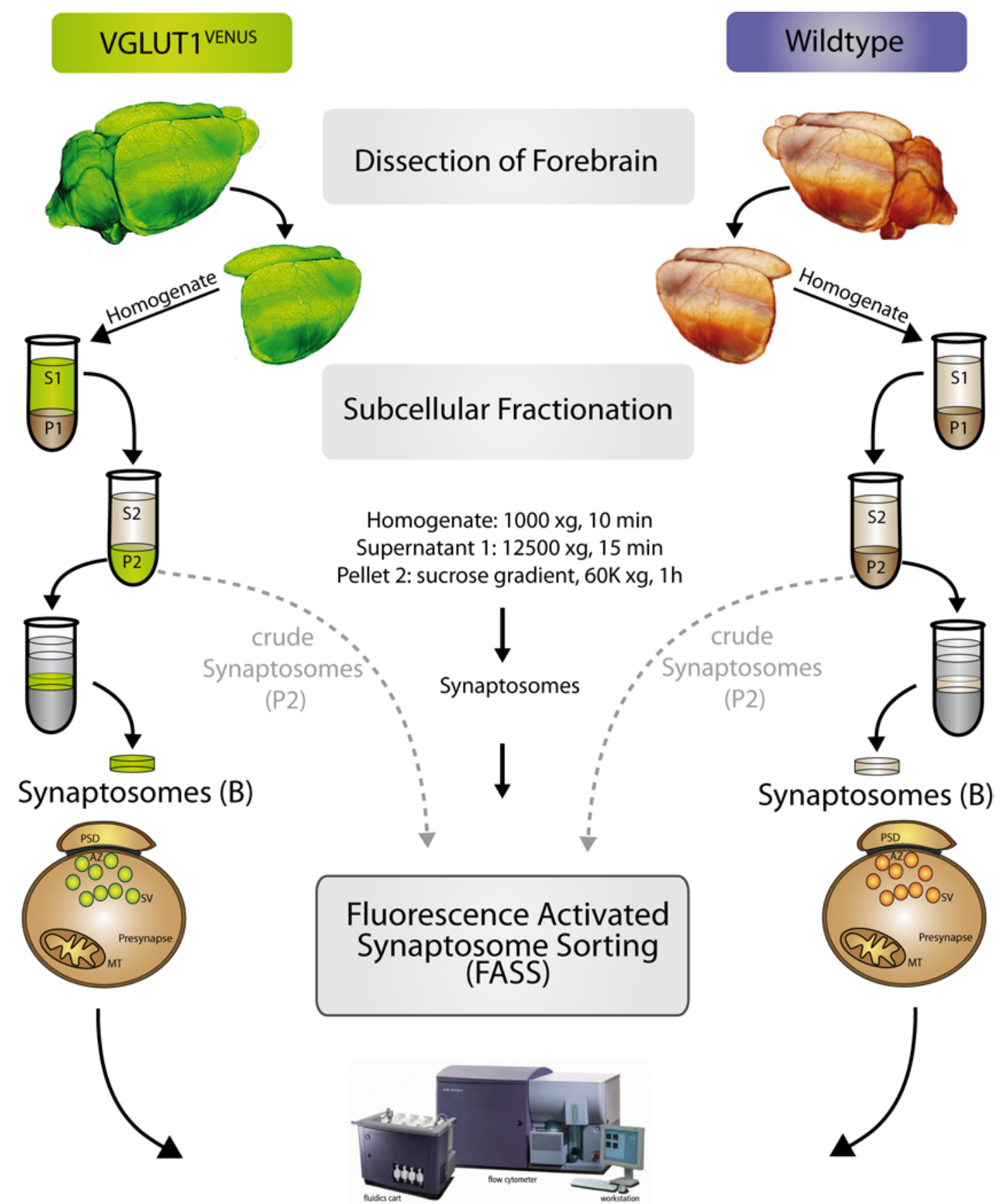

Analyze WT and VGLUT1 ${ }^{\text {venUs }} \mathrm{B}$ and $\mathrm{P} 2$ fraction optimize sorting of VGLUT1 ${ }^{\text {VENUS }}$ Synaptosomes

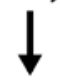

highly pure VGLUT1 1 venUs Synaptosomes after FASS

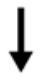

Develop methods for efficient sample recovery for Western blotting and microscopy

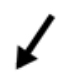

Method validation using Western blotting and microscopy
Extensive Western blot analysis, Proteomics screen, Candidate protein validation 


\section{Materials and Methods}

\subsection{Materials}

\subsubsection{Devices}

\begin{tabular}{|l|l|}
\hline Name & Company \\
\hline & \\
\hline SS-34 rotor & Sorvall \\
\hline Glass-Teflon Potter & B. Braun Biotech International GmbH \\
\hline SW-41 Ti Rotor & Beckman \\
\hline RC-6 Superspeed Centrifuge & Sorvall \\
\hline SS-34 rotor 13.4 mL tubes for adaptors & Sorvall \\
\hline SS-34 rotor centrifuge tube adaptors & Sorvall \\
\hline Vacuum centrifuge (Concentrator 5301) & Eppendorf \\
\hline Microplate centrifuge (Centrifuge 5810R) & Eppendorf \\
\hline Microplate centrifuge rotor (A-4-62) & Eppendorf \\
\hline Microcentrifuge (Centrifuge 5415D) & Eppendorf \\
\hline Microcentrifuge rotor (F45-24-11) & Eppendorf \\
\hline FACSAria-I & Becton-Dickinson \\
\hline Multipette plus & Eppendorf \\
\hline Multichannel pipette (10-100 $\mu \mathrm{L})$ & Eppendorf \\
\hline Multichannel pipette (1-10 $\mu \mathrm{L})$ & Eppendorf \\
\hline Pipette $(0.1-2 \mu \mathrm{L})$ & Gilson \\
\hline Pipette $(1-10 \mu \mathrm{L})$ & Gilson \\
\hline Pipette (2-20 $\mu \mathrm{L})$ & Gilson \\
\hline Pipette $(20-100 \mu \mathrm{L})$ & Gilson \\
\hline Pipette (50-200 $\mu \mathrm{L})$ & Gilson \\
\hline Pipette (100-1000 $\mu \mathrm{L})$ & Gilson \\
\hline Pipette (500 -5000 $\mu \mathrm{L})$ & Gilson \\
\hline Odyssey-system & LI-COR Biosciences \\
\hline Agarose gel imaging system & Intas GmbH \\
\hline Microscope Axio Imager Z.1 & ZEISS \\
\hline Binocular MZ16F, Fluorescence & Leica Microsystems \\
\hline Confocal laser scanning microscope TCS- & Leica Microsystems \\
SP2 & Reichert-Jung \\
\hline Cryotome & Agfa-Gevaert GmbH \\
\hline Developing machine for ECL films & Thermo-Electron \\
\hline$-80{ }^{\circ}$ C Freezers & Adlof Kühne AH, Heraeus GmbH \\
\hline Incubators & Molecular Devices \\
\hline ReadMax Microtiter plate reader & Bio-Rad \\
\hline Mini Protean II system (SDS-PAGE) & Invitrogen \\
\hline Xcell Sure Lock Mini-Cell (SDS-PAGE) & Paul Marienfeld GmbH \\
\hline Neubauer improved cell counting chamber & MJ research, Bio-Rad Laboratories \\
\hline PCR-Thermocycler & GmbH \\
\hline
\end{tabular}




\begin{tabular}{|l|l|}
\hline Name & Company \\
\hline Ultracentrifuge Opitma TLX & Beckman \\
\hline Ultracentrifuge L-60 & Beckman \\
\hline Ultrasonic waterbath & Branson \\
\hline Vacusafe comfort vacuum pump & IBS Integra Biosciences \\
\hline
\end{tabular}

\subsubsection{Consumables and kits}

\begin{tabular}{|l|l|}
\hline Name & Company \\
\hline Modified Bradford assay reagent & Bio-Rad \\
\hline coverslips $\varnothing 13 \mathrm{~mm}$ & Sigma-Aldrich GmbH \\
\hline Syringe-Microfilters 0.22 & Millipore \\
\hline $\begin{array}{l}\text { Isopore } \\
(0.1,0.22,0.45 \mu \mathrm{m})\end{array}$ & Millipore \\
\hline Amersham ECL Hyperfilm & GE-Healthcare \\
\hline $\begin{array}{l}\text { Amersham ECL Western Blotting detection } \\
\text { reagents }\end{array}$ & GE-Healthcare \\
\hline Filter paper Whatman 3MM & Whatman International Ltd. \\
\hline Microscope glass slides & Menzel \\
\hline nexttec ${ }^{\text {TM }}$ Genomic DNA Isolation kit & Nexttec \\
\hline Nitrocellulose Membrane, $0.2 \mu \mathrm{m}$ & Schleicher \& Schuell \\
\hline PCR plates & ThermoScientific \\
\hline Centrifuge tube (Falcon $15 \mathrm{~mL})$ & Greiner Bio-One \\
\hline Centrifuge tube (Falcon $50 \mathrm{~mL})$ & Beckton-Dickinson \\
\hline Centricon-10 & Amicon Inc. \\
\hline Ultracentrifuge tubes & Beckman \\
\hline Filtration Unit .22 $\mu \mathrm{m}(150 \mathrm{~mL})$ & Millipore \\
\hline Filtration Unit .22 $\mu \mathrm{m}(500 \mathrm{~mL})$ & Millipore \\
\hline $\begin{array}{l}\text { NuPAGE Novex Bis-Tris }(4-12 \%) \text { precast } \\
\text { gels }\end{array}$ & Invitrogen \\
\hline NuPAGE Novex MOPS Running Buffer & Invitroogen \\
\hline NuPAGE 4xLDS loading dye & Invitrogen \\
\hline Prestained PAGE-Ruler & Fermentas \\
\hline Microcentrifuge tubes $(0.5,1.5,2.0 \mathrm{~mL})$ & Eppendorf \\
\hline & \\
\hline
\end{tabular}

\subsubsection{Software}

\begin{tabular}{|l|l|}
\hline Name & Company \\
\hline ImageJ 1.43k & http://rsb.info.nih.gov/ij/index.html \\
\hline FACSDiva 6.1.3 & BD Biosciences \\
\hline Microsoft-Office 2004 & Microsoft \\
\hline Illustrator CS3 & Adobe \\
\hline
\end{tabular}




\subsubsection{Chemicals, Reagents, Materials}

\begin{tabular}{|c|c|}
\hline Name & Company \\
\hline Sucrose & Sigma-Aldrich $\mathrm{GmbH}$ \\
\hline Agarose & Life Technologies (Invitrogen) \\
\hline Acetic Acid & Merck \\
\hline Acetone & Sigma-Aldrich GmbH \\
\hline Acetonitrile & Sigma-Aldrich $\mathrm{GmbH}$ \\
\hline $\mathrm{AgNO}_{3}$ & Merck \\
\hline AMBA & BioRad \\
\hline Aprotenin & Roche Diagnostics \\
\hline Ammonium persulfate (APS) & Merck \\
\hline AquaPolyMount & Polysciences \\
\hline Beta-mercaptoethanol & Sigma \\
\hline Bromphenol blue & Pierce \\
\hline $\mathrm{CaCl}_{2}$ & Merck \\
\hline DAPI & AppliChem \\
\hline Dithiotreitol (DTT) & BioMol \\
\hline EDTA & Merck \\
\hline Ethanol & Merck \\
\hline FM4-64 & Invitrogen \\
\hline Formaldehyde (37 \%) & Merck \\
\hline Formic Acid & Sigma-Aldrich GmbH \\
\hline Gelatine & Riedel De Häen \\
\hline Glutaraldehyde & Electron Microscopy Sciences \\
\hline Glycine & Sigma-Aldrich $\mathrm{GmbH}$ \\
\hline HEPES & Sigma-Aldrich $\mathrm{GmbH}$ \\
\hline Iodoacetamide & Sigma-Aldrich $\mathrm{GmbH}$ \\
\hline Isofluran & DeltaSelect \\
\hline $\mathrm{KCl}$ & Merck \\
\hline $\mathrm{KH}_{2} \mathrm{PO}_{4}$ & Merck \\
\hline Leupeptine & Roche Diagnostics \\
\hline Methanol & L.T. Baker \\
\hline $\mathrm{MgCl}_{2} \times 6 \mathrm{H}_{2} \mathrm{O}$ & Merck \\
\hline $\mathrm{Na}_{2} \mathrm{HPO}_{4} \times 2 \mathrm{H}_{2} \mathrm{O}$ & Merck \\
\hline $\mathrm{Na}_{2} \mathrm{~S}_{2} \mathrm{O}_{3}$ & Merck \\
\hline $\mathrm{NaCl}$ & Merck \\
\hline non fat dry milk powder & Gravita GmbH \\
\hline Normal goat serum & Life Technologies (Gibco BRL) \\
\hline Paraformaldehyde & Serva \\
\hline PMSF & Roche Diagnostics \\
\hline Poly-L-Lysine & Sigma-Aldrich $\mathrm{GmbH}$ \\
\hline Ponceau S & Sigma-Aldrich $\mathrm{GmbH}$ \\
\hline RedTaq $^{\text {TM }}$ & Sigma-Aldrich $\mathrm{GmbH}$ \\
\hline RedTaq $^{\text {TM }}$ 10x Buffer & Sigma-Aldrich $\mathrm{GmbH}$ \\
\hline Sodium dodecyl sulfate (SDS) & Sigma-Aldrich GmbH \\
\hline Sucrose & Sigma-Aldrich $\mathrm{GmbH}$ \\
\hline TEAB & Sigma-Aldrich $\mathrm{GmbH}$ \\
\hline TEMED & Serva \\
\hline
\end{tabular}




\begin{tabular}{|l|l|}
\hline Name & Company \\
\hline Tris Base & Sigma-Aldrich \\
\hline Trypsin, sequencing grade (proteomics) & Promega \\
\hline Tween-20 & Sigma-Aldrich GmbH \\
\hline Tri-brom-ethanol & Sigma-Aldrich GmbH \\
\hline
\end{tabular}

\subsubsection{Reagents for cell culture}

\begin{tabular}{|l|l|}
\hline Name & Company \\
\hline PBS (cell culture) & PAA Biochemical Company \\
\hline Papain & $\begin{array}{l}\text { Worthington Biomedical Corporation / } \\
\text { Cell Systems }\end{array}$ \\
\hline Penicillin/Streptomycin & Life Technologies Gibco BRL GmbH \\
\hline Trypsin/EDTA & Life Technologies Gibco BRL GmbH \\
\hline B-27 Supplement & Life Technologies Gibco BRL GmbH \\
\hline Cell culture plastics & Costar, Falcon, Nunc \\
\hline Fetal Calf Serum & Life Technologies Gibco BRL GmbH \\
\hline GlutaMAX TM I & Life Technologies Gibco BRL GmbH \\
\hline Neurobasal medium & Life Technologies Gibco BRL GmbH \\
\hline Poly-L-Lysine & Sigma-Aldrich GmbH \\
\hline PBS (cell culture) & PAA Biochemical Company \\
\hline
\end{tabular}

\subsubsection{Primary antibodies}

\begin{tabular}{|l|l|l|l|l|}
\hline Antibody-Name & Species & Source & $\begin{array}{l}\text { Dilution } \\
\text { WB }\end{array}$ & Dilution IF \\
\hline GFP & $\mathrm{M}$ & Roche & $1: 1,000$ & \\
\hline GFP & $\mathrm{M}$ & Millipore & & $1: 1,000$ \\
\hline GFP (A6455) & $\mathrm{Rb}$ & Invitrogen & & $1: 5,000$ \\
\hline Synaptophysin (clone7.2) & $\mathrm{M}$ & SySy & $1: 10,000$ & \\
\hline Synapsin & $\mathrm{Rb}$ & SySy & $1: 4,000$ & $1: 8,000$ \\
\hline EndophilinA1 (L4P1) & $\mathrm{Rb}$ & $\begin{array}{l}\text { (Vinatier et al., } \\
\text { 2006) }\end{array}$ & $1: 2,000$ & \\
\hline VDAC/Porin & $\mathrm{Rb}$ & Rockland & $1: 1,000$ & \\
\hline IBA1 & $\mathrm{Rb}$ & & $1: 1,000$ & \\
\hline GLT-1 & $\mathrm{GP}$ & Chemicon & $1: 20,000$ & \\
\hline PLP (3F4) & $\mathrm{M}$ & Nave-lab & & \\
\hline VIAAT & $\mathrm{Rb}$ & Chemicon & $1: 250$ & \\
\hline VGLUT1 (53LP1) & $\mathrm{Rb}$ & $\begin{array}{l}\text { EH } \\
\text { (unpublished) }\end{array}$ & $1: 5,000$ & $1: 4,000$ \\
\hline VGLUT1 & $\mathrm{Rb}$ & SySy & & $1: 2,000$ \\
\hline VGLUT1 & $\mathrm{GP}$ & Chemicon & & $1: 5,000$ \\
\hline VIAAT & $\mathrm{Rb}$ & SySy & & $1: 1,000$ \\
\hline VGLUT2 (57LP1) & $\mathrm{Rb}$ & $\begin{array}{l}\text { EH } \\
\text { (unpublished) }\end{array}$ & $1: 2,000$ & \\
\hline VGLUT2 & & Millipore & & $1: 4,000$ \\
\hline
\end{tabular}




\begin{tabular}{|c|c|c|c|c|}
\hline Antibody-Name & Species & Source & $\begin{array}{l}\text { Dilution } \\
\text { WB }\end{array}$ & Dilution IF \\
\hline VAChT & & SySy & $1: 4,000$ & \\
\hline SNAP25 & $\mathrm{M}$ & SySy & $1: 1,000,000$ & \\
\hline SNAP23 & $\mathrm{Rb}$ & SySy & $1: 250$ & \\
\hline SNAP47 & $\mathrm{Rb}$ & SySy & $1: 1,000$ & \\
\hline SV2A & $\mathrm{Rb}$ & SySy & $1: 4,000$ & \\
\hline SV2B & $\mathrm{Rb}$ & SySy & $1: 4,000$ & \\
\hline Complexinl/II & $\mathrm{Rb}$ & Lab & $1: 1,000$ & \\
\hline Neuroligin1 (4c12) & $\mathrm{M}$ & SySy & $1: 8000$ & \\
\hline Neuroligin2 (799) & $\mathrm{Rb}$ & $\begin{array}{l}\text { (Varoqueaux et } \\
\text { al., 2004) }\end{array}$ & $1: 1000$ & \\
\hline Neuroligin3 (804) & $\mathrm{Rb}$ & $\begin{array}{l}\text { (Varoqueaux et } \\
\text { al., 2006) }\end{array}$ & $1: 500$ & \\
\hline Neuroligin4 (75_2-3) & $\mathrm{Rb}$ & $\begin{array}{l}\text { (Jamain et al., } \\
\text { 2008) }\end{array}$ & $1: 500$ & \\
\hline GluRII & $\mathrm{Rb}$ & SySy & $1: 500$ & \\
\hline NR1 & $\mathrm{M}$ & SySy & $1: 500$ & \\
\hline NR2A & $\mathrm{Rb}$ & Chemicon & $1: 1,000$ & \\
\hline NR2B & $\mathrm{Rb}$ & Chemicon & $1: 1,000$ & \\
\hline PSD-95 & $\mathrm{M}$ & BD Transduction & & $1: 1,000$ \\
\hline VGLUT1(53LP1) & $\mathrm{Rb}$ & EH(unpublished) & $1: 5,000$ & $1: 5,000$ \\
\hline Ly6H & $\mathrm{M}$ & Abcam & $1: 500$ & $1: 1,200$ \\
\hline mTPD52 (J.A & $\mathrm{Rb}$ & JA. Byrne & & \\
\hline FXYD6 & $\mathrm{Rb}$ & K. Geering & $1: 2,000$ & $1: 2,000$ \\
\hline MAP2 & $\mathrm{M}$ & Upstate & & $1: 2,000$ \\
\hline MAP2 & $\mathrm{Rb}$ & Chemicon & & $1: 1,000$ \\
\hline GAD65 & $\mathrm{Rb}$ & Chemicon & & $1: 2,000$ \\
\hline GAD67 & $\mathrm{M}$ & Millipore & & $1: 2,000$ \\
\hline GFAP & & $\begin{array}{l}\text { Dako } \\
\text { Cytomation }\end{array}$ & & $1: 500$ \\
\hline ProSAP1 & $\mathrm{Rb}$ & T. Boeckers & & $1: 1,000$ \\
\hline
\end{tabular}

\subsubsection{Secondary antibodies}

\begin{tabular}{|l|l|l|l|l|}
\hline Antibody & Species & Company & Conjugated & Dilution \\
\hline anti-rabbit & Goat & $\begin{array}{l}\text { Jackson-Immuno } \\
\text { Resarch }\end{array}$ & HRP & $\begin{array}{l}1: 2000- \\
1: 5000\end{array}$ \\
\hline anti-mouse & Goat & $\begin{array}{l}\text { Jackson-Immuno } \\
\text { Research }\end{array}$ & HRP & $\begin{array}{l}1: 2000- \\
1: 5000\end{array}$ \\
\hline anti-guinea pig & Goat & BioRad & HRP & $\begin{array}{l}1: 2000- \\
1: 5000\end{array}$ \\
\hline anti-rabbit & Goat & Life Technologies & AL488/555/Cy5 & $1: 1,500$ \\
\hline anti-mouse & Goat & Life Technologies & AL488/555/Cy5 & $1: 1,500$ \\
\hline anti-guinea pig & Goat & Life Technologies & AL488/555/633 & $1: 1,500$ \\
\hline
\end{tabular}




\subsection{Mouse genotyping}

Preparation and handling of DNA was performed according to standard procedures (Sambrook et al., 1989). Polymerase chain reaction (PCR) was originally reported by (Mullis et al., 1986). It is a standard method today and the required thermostable DNA-polymerases are commercially available. The PCR reactions were carried out according to the supplier's recommendations.

\subsubsection{Genotyping PCR}

For genotyping of the VGLUT1 ${ }^{\text {VENUS }}$ knock-in mouse line animals were tested for the presence of the wild type and knock-in allele in two separate PCR reactions. In the wild type PCR, primers 9420 and 9421 are used to amplify a 220 bp (base pair) fragment from exon 12 of the wild type VGLUT1 locus, while the knock-in allele results in a fragment size of $1 \mathrm{~kb}$ (kilo base pairs). However, the short amplification times that are sufficient for production of the 220 bp wild type fragment are not sufficient for reliable production of the knock-in fragment. Therefore, the knock-in (ki) allele is revealed in a separate PCR that results in amplification of a 412 bp fragment using primers 9423 and 9421.

Mouse genomic DNA was isolated from mouse tail samples by the technical assistants of the department of Molecular Neurobiology using the Nextec 3.0 kit according to the manufacturer's recommendations and the PCR reactions were carried out as follows:

\begin{tabular}{|c|c|c|}
\hline \multicolumn{3}{|c|}{ PCR program } \\
\hline Step & Command & (mm.ss) \\
\hline 1 & $94{ }^{\circ} \mathrm{C}$ & 03.00 \\
\hline 2 & $94^{\circ} \mathrm{C}$ & 00.30 \\
\hline 3 & $60^{\circ} \mathrm{C}$ & 00.30 \\
\hline 4 & $72^{\circ} \mathrm{C}$ & $\begin{array}{l}00.40(\mathrm{wt}) \\
01.00 \text { (ki) }\end{array}$ \\
\hline 5 & Go to $2(30 x)$ & \\
\hline 6 & $72^{\circ} \mathrm{C}$ & 07.00 \\
\hline 7 & $10^{\circ} \mathrm{C}$ & $\infty$ \\
\hline
\end{tabular}

\begin{tabular}{|ll|}
\hline \begin{tabular}{l} 
PCR reaction $(20 \mu \mathrm{L})$ \\
\multicolumn{1}{c|}{ Reagent }
\end{tabular} & $\boldsymbol{\mu} \mathrm{L}$ \\
\hline DNA & 1 \\
\hline RedTaq & 1 \\
\hline 10xRedTaq Buffer & 2 \\
\hline Primer1 (5 pM) & 1 \\
\hline Primer2 (5 pM) & 1 \\
\hline dNTPs $(2.5 \mu \mathrm{M}$ each) $)$ & 1 \\
\hline $\mathrm{H}_{2} \mathrm{O}$ & 13 \\
\hline
\end{tabular}

Oligos (numbers refer to MPI-EM AGCT-database):

\# 9420: 5'- CTGGCTGGCAGTGACGAAAG -3'

\# 9421: 5'- CGCTCAGGCTAGAGGTGTATGGA -3'

\# 9423: 5'- CTTCAAGATCCGCCACAACATCG -3' 


\subsubsection{Agarose gel electrophoresis}

\section{TBE BUFFER:}

$50 \mathrm{mM}$ Tris-Base, $50 \mathrm{mM}$ Boric Acid, 2mM EDTA pH8.0

Agarose gel electrophoresis was used to separate the DNA-molecules of the genotyping PCR based on their size according to standard procedures (Voytas, 2000). The RedTaq PCR buffer is very dense and contains a dye to allow direct loading of the PCR reactions into the agarose gel pockets. Gels contained $2 \%$ agarose and $0.5 \mathrm{mg} / \mathrm{mL}$ ethidium bromide. Electrophoresis was carried out using in TBE buffer at constant voltage of 80-120 $\mathrm{V}$. The ethidium bromide stained DNA was visualized under UV-light on an agarose gel imaging system.

\subsection{Subcellular fractionation}

The first isolation of Synaptosomes was reported in 1958 (Hebb and Whittaker, 1958). Since then great variety of protocols for isolation of synaptosomes and synaptosome derived fractions have been developed (Whittaker, 1993). The protocols used in this work have been adapted from protocols described by Hell and Jahn (E. Celis, 1998) and (Huttner et al., 1983). The whole procedure is illustrated in Figure 6.

\section{HOMOGENIZATION BUFFER}

$\mathrm{H}_{2} \mathrm{O}, \quad 0.32 \mathrm{M}$ Sucrose, $4 \mathrm{mM}$ HEPES pH 7.4 Sterile-filtered and stored at $4{ }^{\circ} \mathrm{C}$ for up to 4 weeks. Protease Inhibitors (PMSF, Leupeptin, Aprotinin)

\subsection{AND 1.2 M SUCROSE SOLUTIONS}

$\mathrm{H}_{2} \mathrm{O}, 0.8$ or $1.2 \mathrm{M}$ Sucrose, $4 \mathrm{mM}$ HEPES pH 7.4 Sterile filtered and stored at 4

${ }^{\circ} \mathrm{C}$ for up to 4 weeks. Protease inhibitors (PMSF, Leupeptin, Aprotinin)

LYSIS BUFFER

$\mathrm{H}_{2} \mathrm{O}, 4 \mathrm{mM}$ HEPES pH 7.4. (PMSF, Leupeptin, Aprotinin)

Protease inhibitors Leupeptin and Aprotinin ( $1 \mu \mathrm{g} / \mathrm{mL}$ ) were added to all buffers before each experiment. PMSF $(1 \mu \mathrm{M})$ was added to each buffer directly before use.

\subsubsection{Preparation of gradient purified synaptosomes for FASS}

Mice were sacrificed by cervical dislocation and the brains of one or two mice were removed from the skull and quickly rinsed with PBS. The preparation of the forebrain started by removing the cerebellum and olfactory bulb. Then the two hemispheres were separated 
and the midbrain was pinched out using a pair of bend tweezers. The forebrains were transferred into ice-cold homogenization buffer and protected from light. Making use of a loosely fitting $5 \mathrm{~mL}$ glass-Teflon potter the tissue was homogenized with 12 gentle up-downs in $4 \mathrm{~mL}$ of ice-cold homogenization buffer. After transfer of the homogenate to a centrifuge tube the potter was rinsed with an additional $4 \mathrm{~mL}$ of homogenization buffer. These $8 \mathrm{~mL}$ of homogenate $(\mathrm{H})$ were centrifuged at $1000 \times \mathrm{g}$ for $10 \mathrm{~min}$ at $4{ }^{\circ} \mathrm{C}$ in the SS-34 rotor (with adaptors). The supernatant (S1) is removed carefully from the pellet (P1) and centrifuged at $12500 \times \mathrm{g}$ for $15 \mathrm{~min}$ at $4{ }^{\circ} \mathrm{C}$ in the SS-34 rotor (with adaptors). The supernatant (S2) is removed completely and the synaptosome-enriched pellet $(\mathrm{P} 2)$ is resuspended in $1 \mathrm{~mL}$ of homogenization buffer. The P2 fraction is layered on top of a two-step sucrose density gradient consisting of $5 \mathrm{~mL}$ of $1.2 \mathrm{M}$ and $5 \mathrm{~mL}$ of $0.8 \mathrm{M}$ sucrose. The gradient is centrifuged at ca. $50000 \times \mathrm{g}(16000 \mathrm{rpm})$ for $1 \mathrm{~h} 10 \mathrm{~min}$ at $4{ }^{\circ} \mathrm{C}$ in a SW-41Ti swing-out bucket rotor. Synaptosomes are enriched in the interface of $1.2 \mathrm{M}$ and $0.8 \mathrm{M}$ sucrose and recovered using a $230 \mathrm{~mm}$ Pasteur-pipette. The resulting fraction is referred to as synaptosome fraction or gradient-purified synaptosomes. Aliquots of all fractions were flash-frozen in liquid nitrogen and stored at $-80^{\circ} \mathrm{C}$.

\subsubsection{Preparation of synaptic plasma membranes and crude synaptic vesicles}

The preparation of synaptic plasma membranes and crude synaptic vesicles follows the same basic protocol as used for the preparation of synaptosomes (see above) with the following modifications and additional steps following the preparation of the P2.

Instead of directly loading the P2 onto a sucrose gradient, the P2-pellet was carefully resuspended in $8 \mathrm{~mL}$ of homogenization buffer and transferred into a new centrifuge tube, while avoiding the dark-brown mitochondria rich part of the P2 pellet. After resuspension the sample was centrifuged once more at $12.500 \times \mathrm{g}$ for $15 \mathrm{~min}$ at $4{ }^{\circ} \mathrm{C}$ in the SS-34 rotor. The resulting supernatant $\left(S 2_{1}\right)$ was combined with the first $\mathrm{S} 2$ fraction, mixed and centrifuged at $223,160 \times \mathrm{g}$ for $2 \mathrm{~h}$ at $4{ }^{\circ} \mathrm{C}$ using the Type75Ti fixed angle rotor. This centrifugation yielded the microsomal fraction (P3) and the cytosolic supernatant (S3). While avoiding the mitochondria-rich part, the final P2 fraction was resuspended with $500 \mu \mathrm{L}$ of homogenization buffer and transferred into a loosely fitting glass-teflon potter. Addition of $9 \mathrm{~mL}$ of lysis buffer, followed by homogenization in the potter at $1500 \mathrm{rpm}$ for 3 up-downs was used to the rupture of membrane bound particles and to concomitant release of their microsomal, vesicular and soluble contents. After osmotic lysis, the lysed P2 was centrifuged at 25,000 x g for 20 min in the SS-34 rotor resulting in the "lysed pellet 1" (LP1) and the "lysed supernatant 1" (LS1). The LS1 fraction was centrifuged at 223,160 $\times \mathrm{g}$ for $2 \mathrm{~h}$ in the Type 75Ti fixed angle rotor, resulting in the "lysed supernatant 2" (LS2) and the "lysed pellet 2" the (LP2). The LS1 fraction is enriched in soluble synaptic proteins and was concentrated in Centricon 10 
columns, resulting in $\sim 300 \mu \mathrm{L}$ sample, which was stored for analysis. After removal of the LS2, the LP2 was resuspended in $150 \mu \mathrm{L}$ homogenization buffer and stored for analysis. The LP2 fraction is enriched in synaptic vesicles and referred to as crude synaptic vesicle fraction. The LP1 fraction was resuspended in $1 \mathrm{~mL}$ of homogenization buffer and applied onto a sucrose gradient composed of $5 \mathrm{~mL} 0.8 \mathrm{~mL}$ homogenization buffer on the top of $5 \mathrm{~mL}$ 1.2 M sucrose buffer in a glass tube. The sample was centrifuged at 19,000 rpm (64206 rpm, Beckman L-60 centrifuge, SW41Ti swing out rotor) for $2 \mathrm{~h}$. The upper interphase (LP1A) the lower interphase (LP1B) and the pellet (LP1C) were collected and stored.

\subsection{Flow Cytometry and Fluorescence Activated Synaptosome Sorting}

\subsubsection{FACS instrumentation}

The FACSAria-I of the MPI of experimental Medicine was maintained according to recommendations of the supplier and regularly checked by the Becton-Dickinson technical service. Throughout this study the instrument was operated in two different configurations.

\section{FSC-TRIGGERED CONFIGURATION (FSC-MODE)}

$70 \mu \mathrm{m}$ nozzle

$488 \mathrm{~nm}$ Laser: $\quad$ Area Scaling 1.40, Window Extension 1.0, FSC Area Scaling 1.30.

Parameters: $\quad$ FSC (ND 1.0, 341V), SSC (488/10, 322 V), VGLUT1VENUS (530/30BP, 700V)

FSC threshold: $\quad 5000$ (Operator AND)

Sheath fluid: $\quad$ PBS

Sort Precision: Purity

\section{FM-TRIGGERED-CONFIGURATION (FM-MODE)}

$70 \mu \mathrm{m}$ nozzle

$488 \mathrm{~nm}$ Laser:

Area Scaling 1.40, Window Extension 0.0, FSC Area

Scaling 1.30.

Parameters:

FSC (ND 1.0, 341V), SSC (488/10, 322 V), VGLUT1-

VENUS (530/30BP, 700V), FM4-64 (695/40BP, 700V)

FM4-64 threshold: $\quad 800$ (Operator AND)

Sheath fluid: PBS

Sort Precision: Purity 


\subsubsection{Fluorescence activated synaptosome sorting}

$10 \times P B S(1 L)$

$\mathrm{H}_{2} \mathrm{O}, 80 \mathrm{~g} \mathrm{NaCl}, 2 \mathrm{~g} \mathrm{KCl}, 17.8 \mathrm{~g} \mathrm{Na}_{2} \mathrm{HPO}_{4} \times 2 \mathrm{H}_{2} \mathrm{O}, 2.4 \mathrm{~g} \mathrm{KH}_{2} \mathrm{PO}_{4}$, adjust to $\mathrm{pH}$

7.4 with $\mathrm{HCl}$. Filtered through $.22 \mu \mathrm{m}$ filter

FM4-64 WORKING SOLUTION

$100 \mu \mathrm{g}$ FM4-64 with $100 \mu \mathrm{L}$ 1xPBS

Gradient purified synaptosomes (see 2.3.1) were either directly stored on ice protected from light or first diluted with 2 volumes of either $\mathrm{H}_{2} \mathrm{O}$ or $.75 \%$ Pluronic F-68 solution and stored on ice protected from light. For flow cytometric analysis and sorting synaptosomes were diluted in ice cold PBS supplemented with protease inhibitors (PMSF 1 $\mu \mathrm{M}$, Leupeptin $1 \mu \mathrm{g} / \mathrm{mL}$, Aprotinin $1 \mu \mathrm{g} / \mathrm{mL}$ ). In FM-mode, the lipophilic styryl dye FM4-64 was added to stain all membranous material contained in the sample. The FM4-64 working solution was added in a ratio of $1.5 \mu \mathrm{L} / \mathrm{mL}$. Samples were analyzed and sorted at threshold rates of $20,000-30,000$ evt/s. To maintain sample quality during sorting, a new suspension of synaptosomes was generated every 45-60 min.

In FM-mode single particles were gated by excluding events that showed correlating, high values for FSC-Area and SSC-Area. Events of the single particle gate were sub-gated according to VGLUT1 ${ }^{\text {VENUS }}$ fluorescence intensity. Background fluorescence was determined before each experiment using gradient purified synaptosomes isolated from wild type animals. Thus, VGLUT1 ${ }^{\text {VENUS }}$-positive particles were gated by selecting events with higher than wild type fluorescence (Figure 16).

In FSC-mode single particles were gated by selecting events of low FSC-W and low SSC-W. Events with higher than wild type VGLUT1 ${ }^{\mathrm{VENUS}}$ fluorescence Intensity were subgated from the events of the single particle gate (Figure 11).

To test the success of sorting, sorted particles were reanalyzed by flow cytometry under identical instrument settings. In FM-mode fresh FM4-64 solution was added to the sorted sample before reanalysis.

\subsubsection{Analysis of flow-cytometry data}

Generally flow cytometry data acquisition and data analysis and figure preparation was carried out using the instrument software FACSDiva, provided by Beckton-Dickinson. Throughout this study FACSDiva Software was used in versions 4.1.2 to version 6.0.1.

For further analyses flow-cytometry data was exported as FCS3.0 files. The raw data was extracted from the FCS3.0 files using FCSextract. Using the software Mathematica 7, Dr. Liyi Li of the group of molecular neurobiology performed the logical data transformation 
and the multiple normal distribution component analysis in order to give a precise estimation of the proportions of different particles..

The data transformation in the "logicle data space" way followed the procedures published by Parks et. al, (2006). This data transformation is similar to the "biexponential" data transformation, which is utilized by the FACSDiva software to represent the data in all of the graphs presented in this thesis.

Specifically, the scaling function was given as the following:

$$
\mathrm{S}(\mathrm{x} ; \mathrm{w})=\mathrm{T} \mathrm{e}^{-(\mathrm{m}-\mathrm{w})}\left(\mathrm{e}^{\mathrm{x}-\mathrm{w}}-\mathrm{p}^{2} \mathrm{e}^{-(\mathrm{x}-\mathrm{w}) / \mathrm{p}}+\mathrm{p}^{2}-1\right)
$$

Where the $\mathrm{S}$ is the original signal data and $\mathrm{x}$ is the corresponding transformed value. The $T$ represents the top value of the data, The $W$ is the width of the linearized values in natural log units. The M-value is the breadth of the display in log decades. The p-value. The values of the parameters were chosen in the following way and applied in the transformation of all the data:

The W-value was derived from the largest value of the wild type populations $(\mathrm{W}=0.893747)$.

To allow display of all wild type values and VGLUT1 $1^{\text {VENUS }}$ with the same transformation the $\mathrm{R}$ value was set to $1 \%$.

The T-value was set to the 18 -bit maximum value $(262,144)$

The M-value used was 4.5

Based on the parameters above the p-value was 3.69633

The transformed data was binned in steps of 0.02 in order to allow an appropriate display of the distributions.

Following data transformation data from four independent wild type measurements were fitted as a sum of normal distributions using by minimization of the sum of the fit residues (least $\mathrm{X}^{2}$ fit). A good fitting of the data could be obtained with a minimum of 3 normal distributions. The quality of fitting was tested by visualizing the fit residuals. The fit residuals measure the difference between the fitted function and the raw data for each data point. A random distribution of the difference around $x$-axis indicates the best fit. Using the resulting 4 wild type probability distribution function (PDF) an average wild type-PDF was computed. The three components of the average wild type-PDF were $(0.186,88.7$, $25.1)(0.59,62.4,11.9)(0.23,41.8,11.4)$, where the values are (proportion, mean, deviation).

To model the data from VGLUT1 ${ }^{\text {VENUS }}$ synaptosomes, the wild type-PDF was incorporated as a fixed part to describe the wild type-like, non-fluorescent component in this data. Importantly, the proportion of the wild type PDF to other distributions was not fixed. A good fit of the data of VGLUT1 ${ }^{\text {VENUS }}$ synaptosomes was achieved using the wild type-PDF and a minimum of two additional normal distributions. In this way data of 4 independent 
experiments were fitted and the mean proportion of each of the three components was computed.

\subsubsection{Sample recovery after FASS}

\section{SDS-PAGE SAMPLE BUFFER(3X)}

$140 \mathrm{mM}$ Tris- $\mathrm{HCl}$ (pH 6.8), 3 mM EDTA, $39 \%$ sucrose, $10 \%$ SDS, $0.1 \%$ Bromphenol Blue, $150 \mathrm{mM}$ DTT or $10 \%(\mathrm{v} / \mathrm{v}) \beta$-mercaptoethanol

For analysis by immunoblotting, electron microscopy and proteomic experiments sorted particles were collected and concentrated in a custom build concentrator (Appendix 2 , Figure 13). This device concentrated synaptosomes onto polycarbonate filter discs with a pore size of $0.22 \mu \mathrm{m}$ by vacuum-filtration. The subsequent treatment of the filters for was different for each method. The procedure for electron microscopy is described in the corresponding methods section.

During the sorting experiment a volume of $\min .3-5 \mathrm{~mL}$ was maintained on top of the filter in order to prevent drying of the synaptosomes and in order to allow for efficient cooling in the collection device. After sorting was completed, the walls of the collection device were rinsed once with PBS and subsequently the whole volume was filtered. The filters were removed from the filter cassette holder, placed on a pre-cooled glass plate and cut into pieces of roughly $2 \times 2 \mathrm{~mm}$ using a fresh razor blade. The razor blade was then used to carefully pile up all the pieces by sliding it in between the filter pieces and the glass plate. Using a fine forceps the filter pieces were then transferred into a $2 \mathrm{~mL}$ microcentrifuge cup and flash frozen in liquid-nitrogen. Samples were stored at $-80^{\circ} \mathrm{C}$ until further use.

For elution of proteins the tubes with the filter pieces were shortly placed on ice and then the appropriate volume of 1xSDS-PAGE sample buffer was added. To fully elute, solubilize and reduce synaptosomal proteins bound to the filters, the samples were incubated at $60{ }^{\circ} \mathrm{C}$ for $20 \mathrm{~min}$ under agitation (600 rpm) in a thermo-mixer. In a next step the eluate was transferred into a fresh $1.5 \mathrm{~mL}$ eppendorf cup. To minimize sample loss the remaining eluate is recovered from the filters by centrifugation. To this purpose, the filter pieces are transferred into a $0.5 \mathrm{~mL}$ eppendorf cup, which has been punctured thee times with a $27-\mathrm{G}$ needle at the bottom of the tube. This tube is then placed on top of the $1.5 \mathrm{~mL}$ eppendorf cup containing the eluate and centrifuged at $1000 \mathrm{rpm}$ for $1 \mathrm{~min}$. 


\subsection{SDS-Polyacrylamide gel electrophoresis}

Protein mixtures were separated according to molecular weight by SDSpolyacrylamide gel electrophoresis (PAGE). An excess of negatively charged SDS molecules in the sample buffer binds to, and denatures the proteins. Complete breakup of protein structure requires the reduction of disulfide bridges. The disulfide bridges are reduced is by heating the samples in presence of $\beta$-mercaptoethanol or DTT. Since the native protein charge is outweighed by the negative charge of the SDS molecules, the proteins migrate through the acrylamide gel towards the anode of the electrophoresis chamber. The acrylamide gel functions as a molecular sieve, so that smaller proteins migrate trough the gel faster than larger proteins. Gels with lower concentrations have larger pore sizes and provide better resolution for higher molecular weights, while gels with higher concentrations of acrylamide show better resolution of smaller proteins. The TRIS-glycine system used here was originally described by Laemmli (Laemmli, 1970) and employed with the following specifications:

\section{SEPARATING GEL BUFFER(4X)}

$0.5 \mathrm{M}$ Tris- $\mathrm{HCl}(\mathrm{pH} 6.8), 0.4 \%$ SDS.

STACKING GEL BUFFER(4X)

$1.5 \mathrm{M}$ Tris- $\mathrm{HCl}(\mathrm{pH} 8.8), 0.4 \%$ SDS

SDS-PAGE RUNNING BUFFER(10X)

1L, $144 \mathrm{~g}$ Glycine, $30.3 \mathrm{~g}$ TRIS-Base, add $\mathrm{H}_{2} \mathrm{O}$ to $1 \mathrm{~L}$

SDS-PAGE SAMPLE BUFFER(3X)

$140 \mathrm{mM}$ Tris- $\mathrm{HCl}(\mathrm{pH}$ 6.8), $3 \mathrm{mM}$ EDTA, $39 \%$ sucrose, $10 \%$ SDS, $0.1 \%$

Bromphenol Blue, $150 \mathrm{mM}$ DTT or $10 \%(\mathrm{v} / \mathrm{v}) \beta$-mercaptoethanol

\begin{tabular}{|lcc|}
\hline & separating gel (10 $\mathbf{~ m L})$ & stacking gel (3 $\mathbf{~ m L})$ \\
\hline AMBA (30 \%) & $\begin{array}{c}\text { X mL (for desired } \\
\text { concentration) }\end{array}$ & $0.4 \mathrm{~mL}$ \\
\hline Gel-buffer & $2.5 \mathrm{~mL}$ & $0.75 \mathrm{~mL}$ \\
\hline $\mathrm{H}_{2} \mathrm{O}$ & $10-\mathrm{X} \mathrm{\textrm {mL }}$ & $1.85 \mathrm{~mL}$ \\
\hline APS (10 \%) & 30 & 20 \\
\hline TEMED & 15 & 10 \\
\hline
\end{tabular}

Separating gels contained 8-15 \% AMBA (Acrylamide/N,N'-methylene-bisAcrylamide) in the gel buffer. Stacking gels were composed of $3.75 \%$ bis-acrylamide in the stacking gel buffer. The polymerization of the gels was initiated by addition TEMED (N,N,N',N'-Tetramethylethylenediamine) and ammonium-persulfate (APS). Gels were 
prepared and run using the Bio-Rad - Mini Protean II system. Initially proteins were allowed to migrate into the stacking gel at $10 \mathrm{~mA}$ per gel. Afterwards the current was increased to 25 mA per gel.

In addition, the commercially available NuPAGE of Invitrogen was used according to the manufacturer's protocols. The gels used were either $1.0 \mathrm{~mm}$ or $1.5 \mathrm{~mm}$ Bis-Tris gradient gels of 4-12\%. Gradient gels provide a comparable separation of proteins over a larger range of molecular weights. The MOPS buffer system was used.

\subsubsection{Silver Staining}

Silver staining was performed on the basis of the protocol published by Blum (Blum et al., 1987), with modifications introduced by (Mortz et al., 2001) and the proteomics facility of the MPI-EM.

$\mathrm{NA}_{2} \mathrm{~S}_{2} \mathrm{O}_{3}$-SOLUTION

$\mathrm{H}_{2} \mathrm{O}, 100 \mathrm{~mL}$ with $240 \mu \mathrm{L}$ of $10 \% \mathrm{Na}_{2} \mathrm{~S}_{2} \mathrm{O}_{3}$; added $53 \mu \mathrm{L}$ formaldehyde (37\%)

directly before use.

$\mathrm{AGNO}_{3}-\mathrm{SOLUTION}$

$\mathrm{H}_{2} \mathrm{O}, 100 \mathrm{~mL}$ with $200 \mathrm{mg} \mathrm{AgNO}_{3}$; added $53 \mu \mathrm{L}$ formaldehyde (37\%) directly

before use.

The following procedure was followed to stain proteins in SDS-polyacrylamide gels. All the steps were performed under gentle agitation with $100 \mathrm{~mL}$ freshly prepared solution in clean glass-ware:
o Fixation in $40 \% \mathrm{EtOH}, 10 \%$ acetic-acid
o Two washes for each $20 \mathrm{~min}$ in $30 \% \mathrm{EtOH}$
o One wash for 20 min in $\mathrm{H}_{2} \mathrm{O}$
o Sensitization for $1 \mathrm{~min}$ in $\mathrm{Na}_{2} \mathrm{~S}_{2} \mathrm{O}_{3}$-solution
o Three short 20 second washes in $\mathrm{H}_{2} \mathrm{O}$
o Incubation with $\mathrm{AgNO}_{3}$ - solution for 20 min
o Two short 20 second washes in $\mathrm{H}_{2} \mathrm{O}$
o Development in $\mathrm{Na}_{2} \mathrm{CO}_{3}$ as long as necessary (1-5 min)
o Stop the development by addition of $5 \%$ acetic-acid
o Wash 2x shortly in $\mathrm{H}_{2} \mathrm{O}$

Gels were documented on a conventional flat-bed scanner using transmitted light. 


\subsubsection{Colloidal Coomassie Staining}

Colloidal coomassie staining of proteins in SDS-polyacrylamide gels was carried out using Coomassie Brilliant Blue G-250 with phosphoric acid in the presence of ammonium sulfate according to (Neuhoff et al., 1988; Neuhoff et al., 1985) modified by D. Hesse and O. Jahn of the proteomics group of the MPI-EM.

\section{DYE STOCK SOLUTION:}

$\mathrm{H}_{2} \mathrm{O}, 0.1 \%(\mathrm{w} / \mathrm{v})$ Coomassie Brilliant Blue G250, 2 \% (w/v) ortho-phosphoric acid, $10 \%(\mathrm{w} / \mathrm{v})$ ammonium sulfate, stored at RT for weeks

DYE WORKING SOLUTION:

$80 \%(\mathrm{v} / \mathrm{v})$ dye stock solution and $20 \%$ methanol (v/v), prepared directly before use

All steps are performed on a shaking table at room temperature. Use only glass containers or plastic boxes specified for gel staining. Low quality plastic containers may release plasticizers that interfere with mass spectrometric protein identification.

Fixation of the gel with $40 \%(\mathrm{v} / \mathrm{v})$ ethanol, $10 \%(\mathrm{v} / \mathrm{v})$ acetic acid for at least $60 \mathrm{~min}$ (or overnight)

o Gel was washed in water two times for $10 \mathrm{~min}$

o Gel was incubated in dye working solution overnight

- Gel was transferred into a new container with $1 \%(\mathrm{v} / \mathrm{v})$ acetic acid

o Gel was washed repeatedly with $1 \%$ acetic acid until all Coomassie particles were removed

Gels were documented using a near-infrared fluorescence imager at $700 \mathrm{~nm}$ (Odyssey, LI-COR). For mass spectrometric protein identification sample lanes were cut into 24 individual bands immediately and stored in the appropriate vials/microplates at $-20^{\circ} \mathrm{C}$ until further use. 


\subsection{Determination of Protein concentration}

\subsubsection{Bradford assay}

Marion M. Bradford reported an assay for the measurement of protein concentration, which is based on the colorimetric measurement of the interaction of proteins with the dye Coomassie Brilliant Blue G250 (Bradford, 1976). A modified version of the Bradford assay for measurement of protein concentration is commercially available from Bio-Rad. Using BSA as a reference, this assay was performed in microtiterplates according to the manufacturer's protocols. The dye reaction was monitored after $10 \mathrm{~min}$ using the ReadMax microplate reader at a wavelength of $570 \mathrm{~nm}$.

\subsubsection{In gel titration}

Alternatively, protein amounts were determined relative to a titration series of a comparable protein sample of known concentration. In the case of sorted VGLUT1 ${ }^{\text {VENUS }}$ synaptosomes samples were compared to unsorted gradient purified synaptosomes. Protein amounts were determined by quantifying the intensity of either silver or colloidal coomassie staining after SDS-PAGE.

Quantification after silver-staining was carried out by scanning the gel with transmitted light at a 16-bit depth. Using ImageJ the integrated density of the protein stain was recorded for each lane of the titration series and of one empty lane. The signal of the empty lane determined the background signal, which was subtracted from all values. The

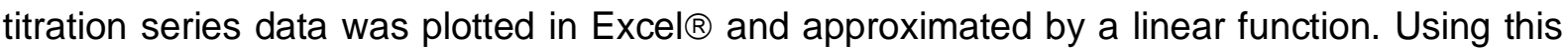
function the protein amount corresponding to the background-subtracted signal of the sample was determined.

Quantification after colloidal coomassie staining was carried out essentially in the same way as described for the silver staining, except that the colloidal coomassie staining was imaged using a near-infrared fluorescence imager (Odyssey ${ }^{\mathrm{TM}}$, LI-COR), which allows reliable quantification of protein amounts over a high dynamic range (Luo et al., 2006) 


\subsection{Proteomics}

\subsubsection{In-gel digestion and mass-spectrometric protein identification}

Following separation by SDS-PAGE, the proteins contained in a sample can be identified by mass-spectrometry. For this, proteins are usually first digested into smaller peptides by treatment with proteases. The serine protease trypsin is most commonly utilized, because for most proteins digestion with trypsin yields peptides in the mass range of 800 $2500 \mathrm{Da}$ which is optimal for MALDI peptide mass fingerprinting and electrospray tandem mass spectrometric sequencing. Protein identity is determined by matching the mass and sequence information of the different peptides to a database containing the theoretically possible tryptic fragments of all proteins in the genome.

In this study, one lane of a polyacrylamide gel containing the proteins to be identified was cut out from the colloidal-coomassie-stained gel and further excised into 24 bands. Each band was cut into cubes of roughly $1 \mathrm{~mm}$ and transferred into a separate well of a digestion plate. The gel pieces were washed twice with $100 \mu \mathrm{L}$ of $50 \%$ acetonitrile in water for 15 min on a shaker. The gel pieces were then dehydrated in $100 \mu \mathrm{L}$ of acetonitrile for $15 \mathrm{~min}$ on a shaker.

For proper identification by mass spectrometry it is important that peptides are not connected via disulfide bonds. Therefore, the acetonitrile was removed and disulfide bonds in the proteins were reduced by swelling the gel pieces in $50 \mu \mathrm{L}$ of $10 \mathrm{mM}$ DTT in $0.1 \mathrm{M}$ TEAB buffer for $45 \mathrm{~min}$ at $56{ }^{\circ} \mathrm{C}$. After removal of excess DTT solution, the formation of new disulfide bridges is prevented by alkylation of the thiol-groups. To this end, the gel pieces were incubated with $50 \mu \mathrm{L}$ of $55 \mathrm{mM}$ iodoacetamide in $0.1 \mathrm{M}$ TEAB buffer for $30 \mathrm{~min}$ protected from light.

After removing the iodoacetemide solution, the gel pieces were washed and dehydrated as before. The gel pieces were rehydrated with $30 \mu \mathrm{L}$ digestion buffer containing $10 \mathrm{ng} / \mu \mathrm{L}$ trypsin in $0.1 \mathrm{M}$ TEAB buffer. In this step the dehydrated gel pieces suck up the trypsin solution like a sponge so that the whole gel volume is exposed to trypsin. To prevent excessive autolysis of trypsin, the rehydration was performed on ice and the remaining trypsin solution was removed after $45 \mathrm{~min}$. Subsequently, $30 \mu \mathrm{L}$ of $0.1 \mathrm{M}$ TEAB buffer were added to keep the gel pieces wet during the overnight digestion at $37^{\circ} \mathrm{C}$.

Following overnight digestion with trypsin, the generated peptides were extracted from the gel pieces. The supernatant of the overnight digestion was collected before addition of $40 \mu \mathrm{L}$ of $5 \%$ fomic acid. An equal volume of acetonitrile was added after $15 \mathrm{~min}$ of shaking. Additional 15 min later the extract was collected. The extraction with formic acid and 
acetonitrile was repeated a second time. In a final extraction with $50 \mu \mathrm{L}$ of acetonitrile the gel pieces were dehydrated completely to maximize peptide recovery. The combined extracts were dried down in a vacuum centrifuge at low or medium heat.

The peptides were redissolved in $30 \mu \mathrm{L}$ of $5 \%$ formic-acid and analyzed by nanoLCMS/MS using a LTQ XL Orbitrap (Thermo Fisher Scientific) coupled to an Agilent 1100 series LC-system. Peptides were separated at a flow rate of $200 \mathrm{~nL} / \mathrm{min}$ on a reversed phase column (C18, Reprosil, Maisch, Germany). Elution of the peptides was done with a 54 minute gradient from 7-45 \% mobile phase B (80 \% acetonitrile, $0.15 \%$ formic acid). Peak lists were searched against NCBI RefSeq database using Mascot v.2.2 as search engine. Mass accuracy was $10 \mathrm{ppm}$ for the parent ion and 0.5 Da for the fragment ions. The peptides were constrained to be tryptic with a maximum of 2 missed cleavages. Carbamidomethylation of cysteines considered a fixed modification, whereas oxidations of methionine and phosphorylation of serine, threonine and tyrosine residues were considered as variable modification.

\subsection{Comparison of proteome and transcriptome data}

In order to validate and further analyze proteomic data a comparison to transcriptome data of Astrocytes, Oligodendrocytes and Neurons published by Cahoy et al. (2008) was carried out. This data analysis was performed in collaboration with Dr. Sven Peter Wichert, from the department of Neurogenetics of the MPI-EM.

\subsubsection{Matching of proteins to Gene Symbols}

To allow retrieval of transcriptome data the proteins identified in the proteomics experiment were first matched to their corresponding Gene-Symbols using the "batchretrieval" function in the framework of the "Protein Information Resource" (http://pir.georgetown.edu/pirwww/search/batch.shtml) (Wu et al., 2003). Unmatched proteins were matched manually using a variety of online databases.

In many cases alternate Gene symbols exist and to retrieve data from Affymetrix microarray experiments the correct gene-symbol for a given Affymetrix chip has to be used. For this reason we mapped our GeneSymbol assignments to the GeneSymbols used on the 430.2 mouse gene expression array of Affymetrix using the id-converter provided by the Babelomics online tool-collection (http://www.babelomics.org) (Al-Shahrour et al., 2008). Remaining mismatches were manually assigned using the NetAffx® Analysis Center of Affymetrix (http://www.affymetrix.com/analysis/index.affx). 


\subsubsection{Analysis, clustering and display of gene expression data as heatmaps}

Using the gene symbols of the proteins analyzed by spectral counting in the present study we retrieved gene expression data generated by Cahoy et al. (2008) from NCBI Gene Expression Omnibus (GSE9566) and normalized using the RMA method. Next we extracted the set of genes corresponding to the proteins that were 2-fold or more enriched in VGLUT1 $1^{\text {VENUS }}$ synaptosomes according to spectral counting - these are the genes of the enriched group. The same was done for the proteins that were 2-fold or more depleted to get the gene expression data for the depleted group. Figure 25 illustrates the algorithm used to cluster the genes. This algorithm was implemented in R (http://www.r-project.org/). First, it was determined if genes are differentially expressed between cell types. A standard deviation of 0.5 or more indicated differential expression. If genes were not differentially expressed they were divided into three groups according to the level of expression (strong, medium or weak). The differentially expressed genes were first grouped according to the cell type with maximal mRNA expression. Genes with maximal expression in neurons were grouped in the Nmax group. Consequently the other groups were designated Amax for maximal expression in astrocytes and Omax for maximal expression in oligodendrocytes. Next, the genes within each group were further sub-clustered according to the relation of the expression in the remaining two cell types. For example in the Nmax group, genes that showed higher expression in oligodendrocytes than in astrocytes were grouped in the NmaxO group. Finally, within these groups genes were ranked according to their average expression level.

After clustering of the genes Partek ${ }^{\circ}$ genomics suite was used to scale the gene expression data and to display the relative gene expression data as a heatmap. 


\subsection{Immuno (Western-) blotting}

Immunodetection of proteins transferred onto nitrocellulose membranes by electroblotting was performed essentially as described in (Towbin et al., 1979). Horseradish peroxidase (HRP) conjugated secondary antibodies were used to allow detection of antigens by enhanced chemiluminescence (ECL).

\section{TRANSFER BUFFER (1L)}

$\mathrm{H}_{2} \mathrm{O}, 3 \mathrm{~g}$ Tris-Base, $14.4 \mathrm{~g}$ Glycine, $200 \mathrm{~mL}$ Methanol

PONCEAU SOLUTION

$\mathrm{H}_{2} \mathrm{O}, 0.1 \%$ Ponceau S, $5 \%$ acetic acid

$10 X \operatorname{TBS}(1 L)$

$\mathrm{H}_{2} \mathrm{O}, 80 \mathrm{~g} \mathrm{NaCl}, 24.2 \mathrm{~g}$ TRIS-base, adjust $\mathrm{pH}$ to 7.4 with $\mathrm{HCl}$

BUFFERA

1xTBS, $5 \%(w / v)$ non-fat dry milk powder, $0.1 \%(v / v)$ Tween-20, $5 \%(v / v)$

normal goat serum

\section{BUFFERB}

1xTBS, $5 \%(w / v)$ non-fat dry milk powder, $0.1 \%(v / v)$ Tween-20

BUFFERC

1xTBS, $5 \%(w / v)$ non-fat dry milk powder

After separation by SDS-PAGE, proteins were transferred to nitrocellulose membranes by a wet-transfer electroblotting method (BIO-RAD Mini- Protean II system). Electroblotting was performed at $50 \mathrm{~mA}$ and $4{ }^{\circ} \mathrm{C}$ for $16 \mathrm{~h}(800 \mathrm{mAh})$ in transfer buffer. Following transfer, proteins were visualized by staining with ponceau solution for 2 min and subsequent 2 washes in $\mathrm{H}_{2} \mathrm{O}$.

Immunodetection of proteins on nitrocellulose membranes was carried out according to the following protocol:

o Unspecific binding sites were saturated by incubation in BufferA for $1 \mathrm{~h}$

o Membranes were incubated with the primary antibody diluted in BufferA for $1 \mathrm{~h}$

o Membranes were washed five times for each 5 min in BufferB

o Membranes were incubated with the secondary antibody (HRP conjugates) diluted in BufferB for $1 \mathrm{~h}$

o Membranes were washed three times for each 5 min in BufferB, twice for 3 min in BufferC, and twice for 3 min in 1xTBS 
Binding of HRP-conjugated secondary antibodies was visualized using a commercially available ECL-detection kit (GE Healthcare) and subsequent exposure of ECLHyperfilm (GE Healthcare) according to the manufacturer's recommendations and developed using a film developer machine (Agfa, Gaevert).

\subsection{Neuronal Cell culture}

Mouse hippocampal cultures were plated onto poly-L-Lysine (PLL) coated, $13 \mathrm{~mm} \varnothing$ coverslips in 24-well cell culture plates or on PLL-coated tissue-culture plastic. Coverslips were cleaned and sterilized by storage in ethanol and subsequent burning of the ethanol under the laminar flow hood. PLL-coating was applied by addition of $0.5 \mathrm{~mL}$ of PLL-solution per well and incubation of minimum $1 \mathrm{~h}$ (usually overnight) at $37^{\circ} \mathrm{C}$ in the cell-culture incubator. Prior to plating of neurons the PLL solution is exchanged for HBSS and plates are incubated for at least $1 \mathrm{~h}$ in the cell-culture incubator, before the HBSS is replaced by NBAcomplete and plates are put back into the incubator until cells are plated.

\subsubsection{Rat primary hippocampal neuron culture}

Rat primary hippocampal neuron cultures were prepared from hippocampi of embryonic day 18 (E18) rats. Cultures were prepared by M Doerre/l Eckhardt as part of their technical assistance to the lab with only minor contributions by me. A pregnant rat was anesthetized with $3 \mathrm{~mL}$ of Isofluran, and sacrificed by decapitation. Fetuses were removed from the abdominal cavity of the rat, decapitated and brains were removed on ice. The hippocampi were dissected in HBSS medium. Between 2 and 10 hippocampi were placed into a $15 \mathrm{~mL}$ falcon tube containing $5 \mathrm{~mL}$ of HBSS medium. They were rinsed 3 times with 10 $\mathrm{mL}$ of HBSS medium. $500 \mu \mathrm{L}$ of trypsin was added and digestion carried out for $15 \mathrm{~min}$ at $37^{\circ} \mathrm{C}$ followed by four washes with each $10 \mathrm{~mL}$ of HBSS medium. Hippocampi were transferred in a $1.5 \mathrm{~mL}$ eppendorf cup with $1 \mathrm{~mL}$ DMEM and triturated twice using a pasteur pipette. After cell clumps had sedimented, the supernatant was filtered through a $40 \mu \mathrm{m}$ cell strainer and collected in a $50 \mathrm{~mL}$ Falcon tube. The hippocampi were then triturated with 4 updowns using a $25 \mathrm{G}$ needle ( $25 \mathrm{G} \times 1^{\prime} / 0.5 \mathrm{~mm} \times 25 \mathrm{~mm}$ ) and twice using a $30 \mathrm{G}$ needle (30G $\times 0.5 / 0.3 \mathrm{~mm} \times 13 \mathrm{~mm}$ ), after each trituration the supernatant was filtered and collected in the same tube. The cell strainer was washed once with $1 \mathrm{~mL}$ of DMEM and the falcon tube was completed with $20 \mathrm{~mL}$ of DMEM. Cells were counted in a $0.100 \mathrm{~nm}$ improved Neubauer chamber. Cells were brought to a concentration of 60,000 cells $/ \mathrm{mL}$ and plated onto poly-L-Lysine coated $12 \mathrm{~mm}$ coverslips in a 24-well plate. $0.5 \mathrm{~mL}$ of the cell suspension was added to each well and cells were grown for 22 days at $37{ }^{\circ} \mathrm{C}, 5 \% \mathrm{CO}_{2}$. 


\subsubsection{Mouse primary hippocampal neuron culture}

\section{PAPAIN SOLUTION}

DMEM, $0.2 \mathrm{mg} / \mathrm{mL}$ Cysteine, $10 \mathrm{mM} \mathrm{CaCl}$, $5 \mathrm{mM}$ EDTA, $5 \mathrm{U} / \mathrm{mL}$ Papain, supplied with Carbogen for at least 20 min

\section{STOP SOLUTION}

FBS-medium, $2.5 \mathrm{mg} / \mathrm{mL}$ BSA, $2.5 \mathrm{mg} / \mathrm{mL}$ Trypsin inactivator

NBA complete: NBA medium, 1x B27, 2 mM GlutaMAXTMI, 20 U/mL Penicillin, 20 $\mu \mathrm{g} / \mathrm{mL}$ Streptomycin

\section{NBA COMPLETE}

NBA medium, 1x B27, $2 \mathrm{mM}$ GlutaMAX'M I, $20 \mathrm{U} / \mathrm{mL}$ Penicillin, $20 \mu \mathrm{g} / \mathrm{mL}$ Streptomycin

\section{FBS-MEDIUM}

DMEM, 10 \% FBS, $500 \mu \mathrm{L}$, MITO+ Serum Extender (1 ampulla/5 mL DMEM), mL $20 \mathrm{U} / \mathrm{mL}$ Penicillin

\section{PLL-SOLUTION}

$11 \mathrm{~mL}$ PBS, $1 \mathrm{~mL}$ Poly-L-Lysine

Brains of newborn mice were quickly removed and collected in ice-cooled HBSS. Hippocampi were dissected and transferred to $0.5 \mathrm{~mL}$ Papain solution, pre-warmed at $37^{\circ} \mathrm{C}$, and incubated for 1 hour at $37^{\circ} \mathrm{C}$ with gentle agitation (600 rpm, Thermo mixer). The Papain solution was exchanged for $0.5 \mathrm{~mL}$ pre-warmed Stop solution and the hippocampi were incubated for $15-20$ minutes at $37^{\circ} \mathrm{C}$ with gentle agitation. Next the complete supernatant was removed and $200 \mu \mathrm{L}$ pre-warmed NBA complete were added. The hippocampi were gently triturated 10-20 times with a 10-200 $\mu \mathrm{L}$ pipette tip. Remaining tissue clumps were sedimented by a short incubation of 1-2 min and then the supernatant was transferred to 1 $\mathrm{mL}$ of pre-warmed NBA complete. Live cells were distinguished from debris by their increased light diffraction (corona), counted using an improved Neubauer chamber and plated. The seeding density was 30,000 cells per well of a 24 -well plate (for imaging) and 150,000-300,000 per 6-cm culture dish (for biochemistry). The medium was not changed. 


\subsection{Electron Microscopy}

\subsubsection{Electron microscopy of synaptosomes}

\section{SYNAPTOSOME REGENERATION BUFFER:}

$\mathrm{H}_{2} \mathrm{O}, 64 \mathrm{mM} \mathrm{NaCl}, 4 \mathrm{mM} \mathrm{KCl}, 0.8 \mathrm{mM} \mathrm{CaCl}, 0.8 \mathrm{mM} \mathrm{MgCl} 28 \mathrm{mM}$ Tris- $\mathrm{HCl}(\mathrm{pH}$ 7.4), $160 \mathrm{mM}$ Sucrose.

For electron microscopy sorted particles were collected onto polycarbonate filter discs. The filters were then incubated with preheated synaptosome regeneration buffer (37 ${ }^{\circ} \mathrm{C}$ ) at room temperature for $20 \mathrm{~min}$, inside the concentrator. The synaptosome regeneration buffer was removed by vacuum filtration and the filter was immediately fixed in $2.5 \%$ Glutaraldehyde in PBS, for 30 min on ice. The filters were washed three times for 10 min in PBS on ice and then stored at $4{ }^{\circ} \mathrm{C}$ overnight before being processed for electron microscopy according to the following procedure:

Osmium-tetroxide fixation, dehydration and Epon embedding, cutting and contrasting were carried out by M. Doerre according to the following protocol:

o Three washes of 3, 8 and 8 min in PBS

o Fixation in $1 \%$ Osmiumtetroxide in PBS

o Three washes of 5, 8 and 8 min in PBS

o 10 min in $30 \% \mathrm{EtOH}$

o 10 min in $50 \% \mathrm{EtOH}$

o 1 h30 min in $70 \%$ EtOH with $1.5 \%$ Uranyl-Acetate

o 8 min in $90 \%$ EtOH

o Three dehydration steps for 3, 8 and $8 \mathrm{~min}$ in $100 \%$

o 8 min in $100 \% \mathrm{EtOH}$, water-free

o 20-25 min in EtOH:Epon (1:1) in a closed container

o $1 \mathrm{~h}$ in EtOH:Epon (1:1) in an open container

o Incubation in pure Epon over-night

o Transfer of filter pieces in embedding molds

o $24 \mathrm{~h}$ polymerization at $60^{\circ} \mathrm{C}$ 
For analysis of synaptosomes after FASS, $70 \mathrm{~nm}$ ultrathin sections were cut from three distinct locations of the filter membrane, and 2 sections of each location were contrasted and imaged using a LEO912AB transmission electron microscope (Zeiss). Digital images were taken using a ProScan CCD camera and operated through the AnalySIS software (Olympus). Following export Images were processed and quantified using ImageJ software. Imaging and Image analysis/quantification were performed in a "blind-observer" fashion, meaning that the identity of the samples was coded and was only revealed after the full analysis had been completed. For quantification images were opened in Image $\mathrm{J}$ and synaptic and debris particles were each delineated using a polygon shape. The number of delineated structures as well as their area was measured.

\subsubsection{Pre-embedding immuno-EM on ultrathin sections}

Pre-embedding immuno-EM of ultrathin sections using the FXYD6 antibody was performed in collaboration with Veronique Bernard according to their published procedures. (Dobbertin et al., 2009)

\subsection{Immunofluorescence labeling and microscopy}

Immunofluorescence labeling and microscopy were carried out according to standard procedures. Confocal images were taken on a confocal laser scanning microscope TCSSP2. Epifluorescence microscopy was performed either on an epifluorescence microscope Axio Imager Z.1 (Zeiss) or fluorescence Binocular MZ16F (Leica).

\subsubsection{Cultured Neurons}

Neuronal cultures were grown on 12-mm poly-L-lysine coated glass coverslips in 24well plates. Before fixation the cultures were washed twice using PB-Sucrose buffer. Cells were fixed in $4 \%$ PFA/PB-Sucrose for $20 \mathrm{~min}$ on ice. After fixation the coverslips were washed three times with PBS and either used directly in immunofluorescence staining or stored in PBS containing $0.02 \%$ sodium-azide at $4{ }^{\circ} \mathrm{C}$.

The coverslips were removed from the 24-well plates and placed on parafilm. To wash of the sodium-azide coverslips were rinsed twice with $100 \mu \mathrm{L}$ of PBS. In order permeabilize the cells for the cells for the antibody and to avoid unspecific binding of the antibody, cultures were incubated in $50 \mu \mathrm{L}$ of PGT for $30 \mathrm{~min}$ to $1 \mathrm{~h}$. The primary antibody was also prepared in PGT and incubated for $1 \mathrm{~h}$ at RT or at $4{ }^{\circ} \mathrm{C}$ overnight. Following five 5minute-washes with PBS the fluorescently coupled secondary antibody was prepared in PGT and incubated for $1 \mathrm{~h}$. Nuclei are stained using $0.1 \mu \mathrm{g} / \mathrm{mL}$ DAPI in PBS for $10 \mathrm{~min}$. Finally, 
coverslips were washed 4-times with PBS and then mounted onto glass objective slides using AquaPolyMount.

\subsubsection{Synaptosomes}

To allow the efficient fixation of synaptosomes to the surface of 12-mm glass coverslips, they were gelatinized by incubation for several hours in .22 $\mu \mathrm{m}$ filtered $1 \%$ gelatin solution. After gelatinization coverslips were rinsed shortly in PBS and then placed into the wells of custom made centrifuge adaptors (Appendix 3). $3 \mathrm{~mL}$ of each sample of the FASS procedure were pipetted on top of each coverslip. Particles were then pelleted onto the glass coverslip by centrifugation at $10,000 \times \mathrm{g}$ for 20 min using the JA7.5 swing-out bucket rotor in a Beckman JS-2 centrifuge. After centrifugation the coverslips were transferred into 24-well plates, which already contain $500 \mu \mathrm{L}$ of $4 \%$ PFA/PB-Sucrose per well. Fixation was carried out for 20 min on ice. The coverslips were then washed three times in PBS and otherwise treated in the same way as described for cultured neurons in the preceding paragraph

\subsubsection{Brain sections}

$\operatorname{AVERTIN}(100 X)$ :

$25 \mathrm{gr}$ tribromoethanol in $15.62 \mathrm{~mL}$ tert-amyl alcohol)

$10 \times P B S(1 L)$

$\mathrm{H}_{2} \mathrm{O}, 80 \mathrm{~g} \mathrm{NaCl}, 2 \mathrm{~g} \mathrm{KCl}, 17.8 \mathrm{~g} \mathrm{Na}_{2} \mathrm{HPO}_{4} \times 2 \mathrm{H}_{2} \mathrm{O}, 2.4 \mathrm{~g} \mathrm{KH}_{2} \mathrm{PO}_{4}$, adjust to $\mathrm{pH}$

7.4 with $\mathrm{HCl}$. Filtered through $.22 \mu \mathrm{m}$ filter

Mice were deeply anesthetized by intraperitoneal injection of 0.3-0.5 mL Avertin. Once in deep anesthesia, the animal was stretched out on its back by fixing fore- and hind limbs with needles. Next the skin was incised and the body cavity opened. The diaphragm was cut and the ribcage opened to gain access to the heart. After opening the right atrium a needle was injected into the left ventricle. Using a peristaltic pump the animal was first perfused with PBS in order to remove blood from the blood vessels. After the blood has been removed, perfusion is continued using $4 \%$ PFA in PBS. Animals are perfused for about 10 min using approximately $200 \mathrm{~mL}$ of PFA solution. Afterwards, brains were removed from the skull and post-fixed in $4 \%$ PFA-PBS for $2 \mathrm{~h}$. For cryopreservation brains are incubated in increasingly concentrated sucrose solutions. First brains were incubated in $10 \%$ sucrose, which was exchanged for $30 \%$ sucrose when brains had sunk to the bottom of the tube. Brains were frozen in Isopentane at -35 to $-40{ }^{\circ} \mathrm{C}$ and stored at $-80^{\circ} \mathrm{C}$. Before sectioning the brains were brought to a temperature of $-20{ }^{\circ} \mathrm{C}$ mounted using TissueTek and subsequently cut into $40 \mu \mathrm{m}$ sections on a cryotome. 


\subsection{Image analysis using ImageJ}

\subsubsection{VGLUT1 ${ }^{\text {VENUS }}$ synaptosome colocalization assay}

Dual color images were obtained by imaging of synaptosomes attached to gelatinized coverslips on and Axiovert Z.1 epifluorescence microscope. Proper alignment of the images in different channels was confirmed using multicolor fluorescent beads of $1 \mu \mathrm{m}$ size (TetraSpeck beads). Using ImageJ I analyzed the immunofluorescence staining of marker proteins in VGLUT1 ${ }^{\text {VENUS }}$ synaptosomes after FASS.

The image analysis in Image J followed the following sequence:

o Open the two-channel image (VGLUT1 ${ }^{\text {VENUS }}$ channel and Channel2)

o The displayed grey values were set to the full 12-bit range of 0-4095

o Noise was reduced using the despeckle command

o Applied Maximum-Entropy threshold to both channels - only signals above the threshold remained

0 Binarized the images and dilated by $2 \mathrm{px}$

o Watershed

At this point two binary images display the signals above threshold in each channel binaryVGLUT1 $1^{\text {VENUS }}$ and binary Channel2.

o Using image calculator the two binary images were added with logic operator AND

0 This final image contained signal only in those regions where the two signals were overlapping and therefore represents particles with colocalization.

In a next step, using the particle analyzer function in ImageJ I counted the number of particles in the binaryVGLUT1 $1^{\text {VENUS }}$ image and the number of particles in the final colocalization image. 


\section{$3 \quad$ Results}

As outlined in the introduction, Synaptosomes have been used extensively to characterize the biochemical composition and functional characteristics of synapses in physiology and pathology (Whittaker, 1993). However, the heterogeneity of the synaptosome preparation makes it difficult to interpret synaptosome-derived data in many contexts. Consequently, for many years there has been a need for protocols that allow a further purification of synaptosomal particles. Therefore in the present study, we make use of the recently developed VGLUT1 ${ }^{\text {VENUS }}$ mouse model (see introduction) to develop methods that allow the purification of VGLUT1 specific synaptosomes for further biochemical analyses.

The following results section contains a considerable amount of flow-cytometry data. In neuroscience flow-cytometry is not a technique applied by many on a regular basis and also most relevant publications in the field only contain limited amounts of flow-cytometry data. For this reason the Appendix 1 contains a short description of the different parameters analyzed in my work and briefly describes how they are presented here. While this description may be unnecessary for some readers I hope it will facilitate reading the flowcytometry section for others.

\subsection{Use of gradient purified synaptosomes (B) results in faster sorting and higher purity compared to crude synaptosomes (P2)}

Previously published studies on flow-cytometry and fluorescence activated sorting of synaptosomes employed different subcellular fractions as starting material. While Wolf and colleagues used gradient purified synaptosomes (B) (Wolf and Kapatos, 1989a; Wolf and Kapatos, 1989b), Gylys and colleagues preferred the use of P2 fractions (Gylys et al., 2000; Gylys et al., 2004a). The preference for using the P2 or the B fraction depends on two key parameters. Firstly, the initial degree of purification of fluorescent synaptosomes might influence the speed of sorting as well as the yield and the purity of sorted synaptosomes. Secondly, the time passing between homogenization of the brain tissue and the sorting step might influence the sample quality. I therefore compared the FASS method with crude synaptosomes (P2; Figure 10) and gradient purified synaptosomes (B; Figure 11) as starting material.

During the preparation of synaptosomes, synaptic particles are already pre-enriched in the post-nuclear pellet P2. In my protocol the preparation of the P2 requires a combined 25 min of centrifugation time (Figure 6). From the P2, synaptosomes are further enriched by 
discontinuous sucrose-density gradient centrifugation. The density-gradient purification adds more than one hour of centrifugation time to the protocol (2.3.1, Figure 6).

When analyzing subcellular fractions by flow cytometry, I analyzed the following parameters: FSC- forward angle light scattering, SSC - $90^{\circ}$-angle light scattering also called side scatter and VGLUT1 $1^{\text {VENUS }}$ fluorescence. The signals measured for each of these parameters consists of three components, namely the height $(H)$, the width $(W)$ and the area (A) of the signals (for further explanations see Appendix 1).

The VGLUT1 ${ }^{\text {VENUS }}$ P2 preparation contained $38.3 \%$ particles above wild-type background fluorescence (Figure $10 \mathrm{~A}$ ). In order to avoid the analysis of aggregated particles and particles that are coinciding at the time of detection I selected particles of low SSC-W and FSC-W in the "small singles" gate (see Figure 10) for gate definition). After this gating step $7 \%$ of all VGLUT1 VENUS P2-particles had a fluorescence signal above the wild-type background and were selected using the "fluorescent" gate (Figure $10 \mathrm{~B}$ ). For simplicity, the term 'fluorescent particles' will refer to the fluorescent events after the gating step that avoids aggregates.

After sorting of the fluorescent particles of the P2, the sorted particles can be reanalyzed using the FACSAria (Figure $10 \mathrm{D}$ ). In order to control for unspecific effects of the sorting step on the sample, I also sorted and re-analyzed all P2-particles non-selectively (Figure $10 \mathrm{C}$ ). The re-analysis showed that sorting the fluorescent particles results in a more homogenous and smaller particle size and complexity distribution as indicated by the forward- and side-scatter analysis (FSC-A/SSC-A in Figure $10 \mathrm{D}$ ). In the fluorescent sample $35.9 \%$ of particles were within the "ungated fluorescence" gate (Figure $10 \mathrm{D}$ ). The "ungated fluorescence" gate includes all particles that are above the wild-type background fluorescence irrespective of their light-scatter (FSC/SSC) signals. When all particles were sorted and re-analyzed, only $11.7 \%$ of particles were in the "ungated fluorescent" gate (Figure $10 \mathrm{C}$ ). Thus, sorting of the fluorescent events of the P2-fraction resulted in a 3.1-fold enrichment of fluorescent particles, but only reached a total of $35.9 \%$ fluorescent particles after FASS. 


\section{A}

unsorted WT crude synaptosomes (WT-P2)
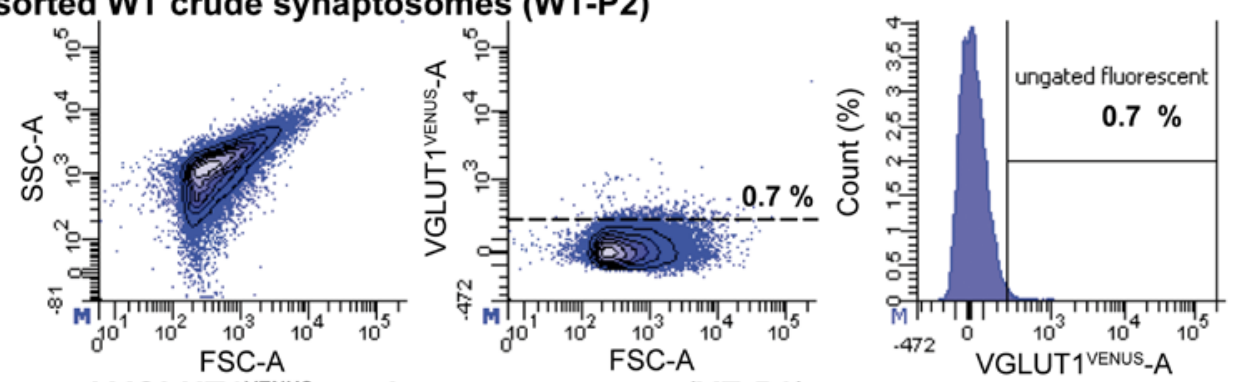

unsorted VGLUT1 ${ }^{\text {VENUS }}$ crude synaptosomes (VE-P2)
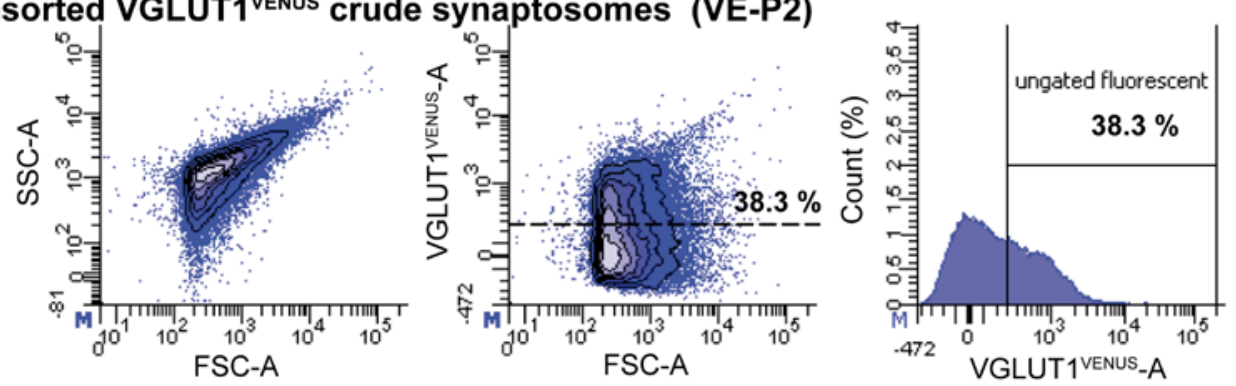

B

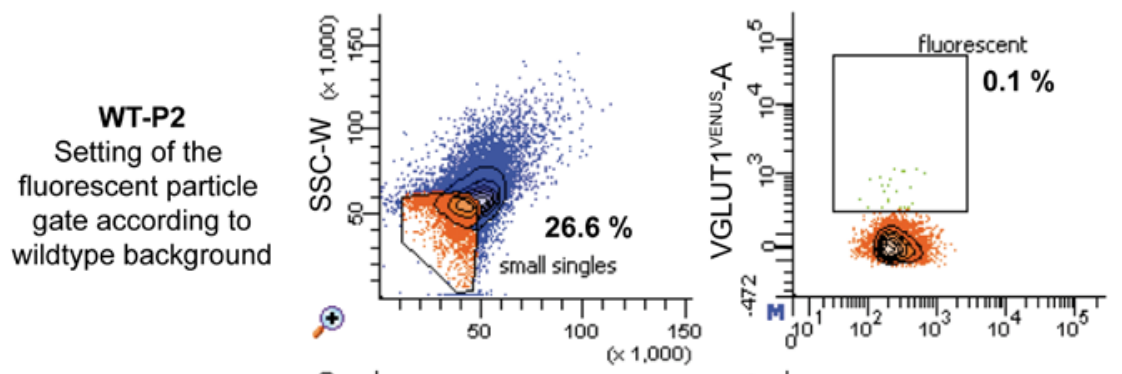

VE-P2

Gates for sorting of single fluorescent particles
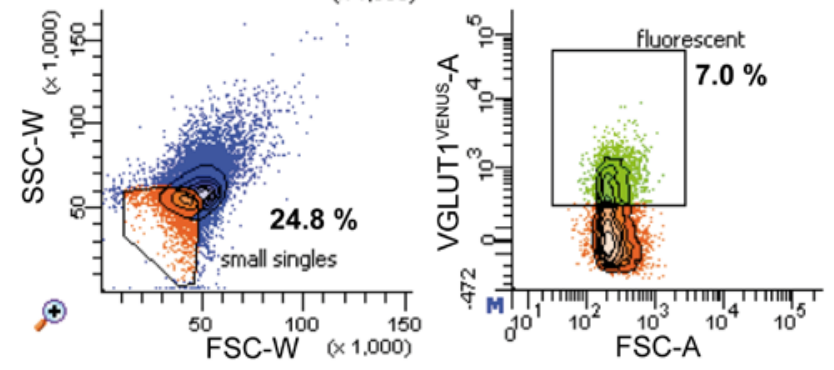

C Reanalysis of sorted VE-P2 - All Particles
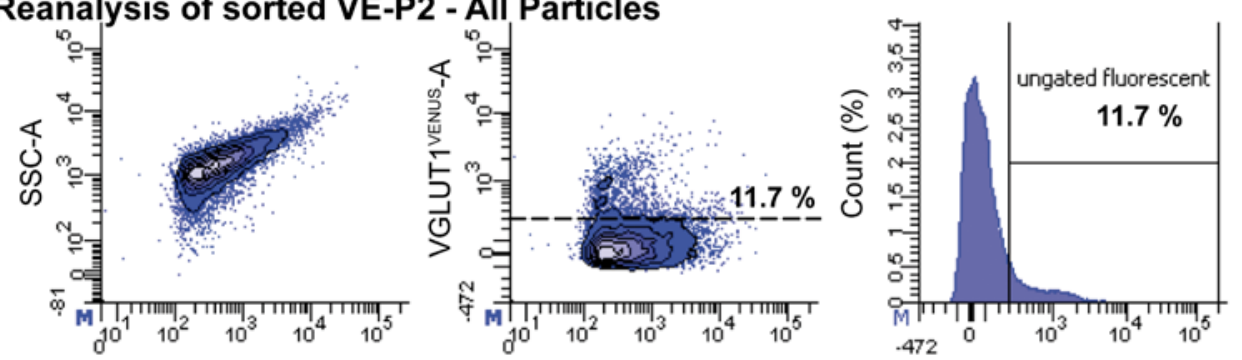

D Reanalysis of sorted VE-P2 fluorescent particles
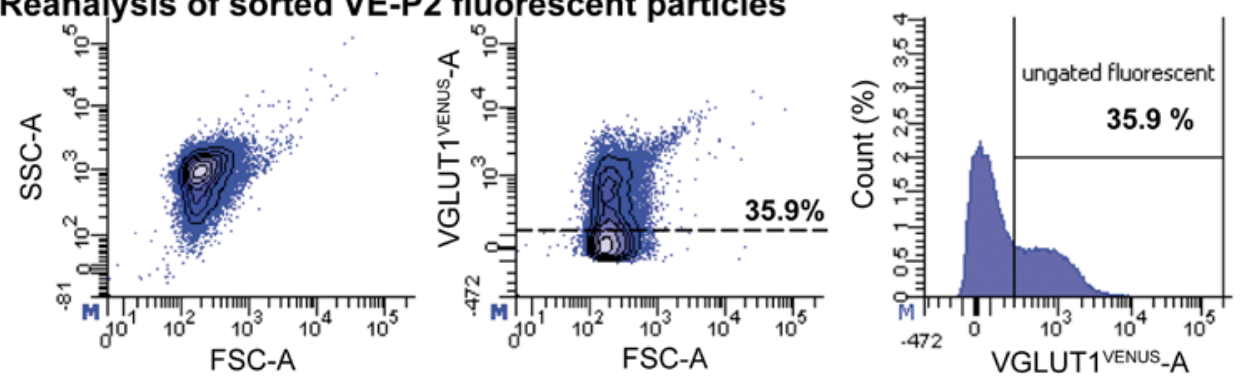


\section{Figure 10: FASS of VGLUT1 ${ }^{\text {VENUS }}$ crude Synaptosomes (P2)}

Flow analysis and sorting of crude synaptosomes from wild type and VGLUT1 ${ }^{\text {VENUS }}$ crude synaptosomes (P2). (A) Analysis of wild type and VGLUT1 ${ }^{\text {VENUS }}$ crude synaptosomes before sorting. The left plots show the analysis of light scattering in forward angles (FCS) and at $90^{\circ}$ angle (SSC), which represent the relative size and internal complexity of the particles, respectively. The central plots display the relative size (FSC) and the fluorescence intensity in the VENUS channel. On the right the fluorescence intensity distribution of the P2 samples of wild type and VGLUT1 ${ }^{\text {VENUS }}$ P2 particles is represented in a histogram. (B) Gating strategy to preferentially select single particles above wild-type background fluorescence. In a first step a gate was drawn to include particles of small SSC-width and FSC-width in order to preferentially select single events. Next, the particles in this gate were analyzed for their fluorescence in the VENUS channel, a gate was drawn to include particles above wild-type background fluorescence. (C) Flow cytometric re-analysis of sorted P2 particles from VGLUT1 ${ }^{\text {VENUS }}$ mice. In this experiment all P2 particles were sorted and reanalyzed. (D) Flow cytometric re-analysis of sorted, fluorescent P2 particles from VGLUT1 ${ }^{\text {VENUS }}$ mice. In this experiment particles, which fell into the fluorescent gate (see VE-P2 in (B)), were sorted and re-analyzed.

When gradient-purified VGLUT1 ${ }^{\text {VENUS }}$ synaptosomes were used as starting material for FASS, $56 \%$ of all particles were above wild-type background fluorescence and $14.7 \%$ of particles sample fell into the single particle fluorescent gate (Figure $11 \mathrm{AB}$ ). The re-analysis of sorted material revealed that, when all particles were sorted and re-analyzed $45.8 \%$ of the events were above wild-type background fluorescence (Figure $11 \mathrm{C}$ ). In contrast, when the fluorescent particles of the B-fraction were sorted and re-analyzed $78.4 \%$ of the particles were detected as fluorescent (Figure 11). Thus, sorting from gradient-purified VGLUT1 VENUS synaptosomes resulted in a 1.7-fold enrichment of fluorescent particles and reached a total of $78.4 \%$ fluorescent particles after FASS.

Additionally, the B-fraction contained twice more (14.7\%) single, fluorescent events than the P2-fraction (7.0\%). This increase in the fraction of positive events, resulted in twice faster sort rates during fluorescence activated sorting in the FACSAria, resulting in a sort time of roughly 60 min for 10 million fluorescent particles when sorting from $B$.

In summary, the preparation of gradient-purified synaptosomes is much more time consuming, but results in a 2.2-fold higher purity of VGLUT1 ${ }^{\text {VENUS }}$ synaptosomes as analyzed by flow cytometry. In addition, the higher fraction of positive particles in the B-fraction allows for faster sorting, which decreased the total time for isolation of larger numbers of VGLUT1 ${ }^{\text {VENUS }}$ synaptosomes by FASS. 
A unsorted WT synaptosomes (WT-B)
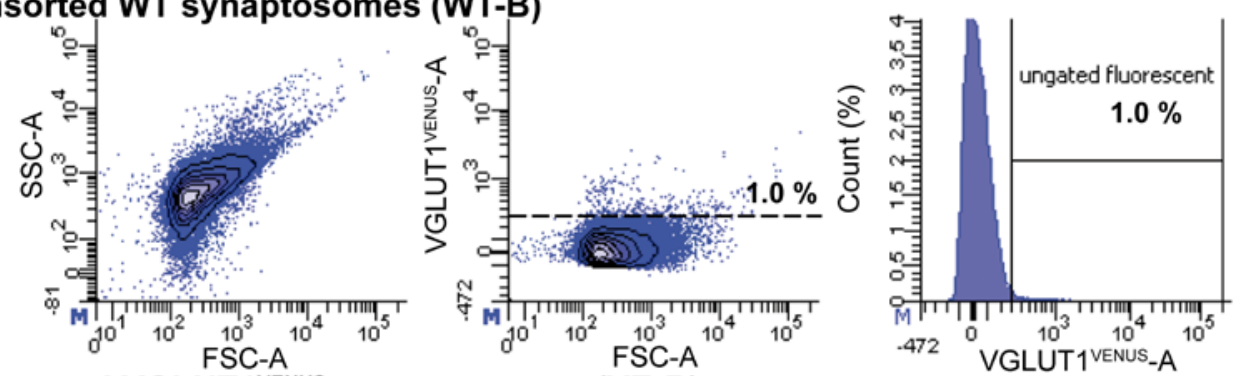

unsorted VGLUT1 $1^{\text {VENUS }}$ synaptosomes (VE-B)
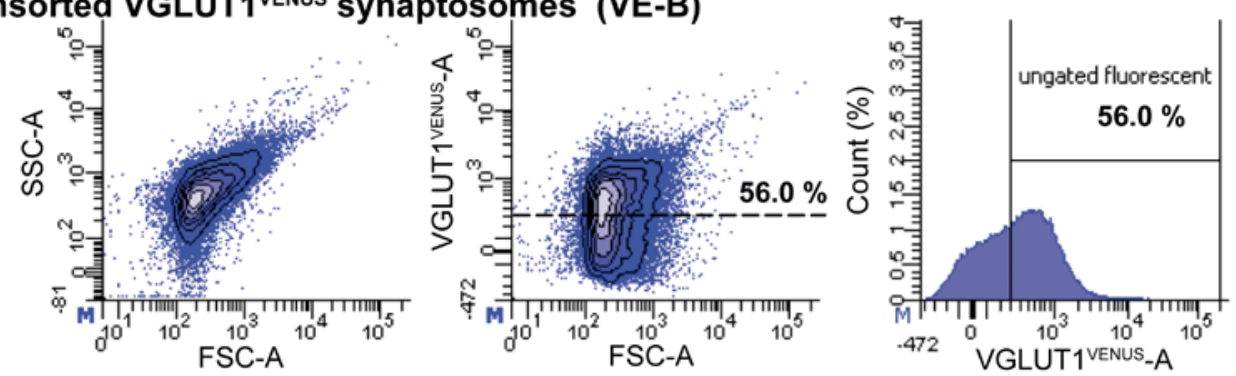

B
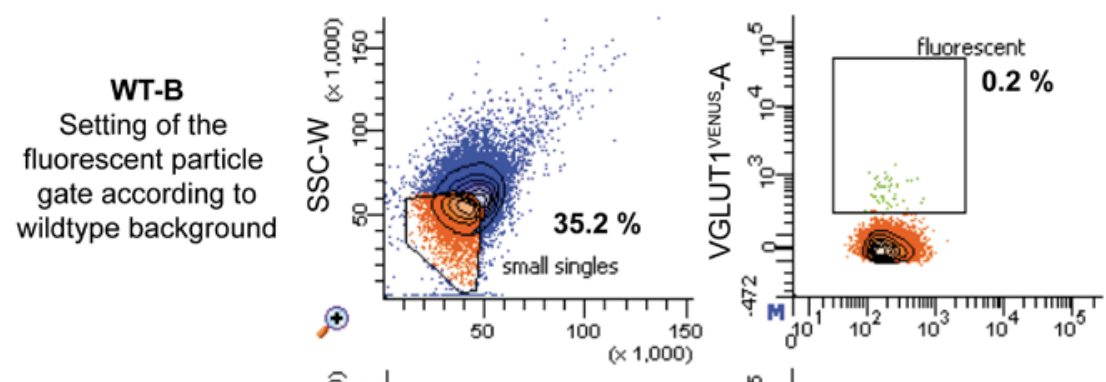

VE-B
Gates for sorting of single fluorescent particles
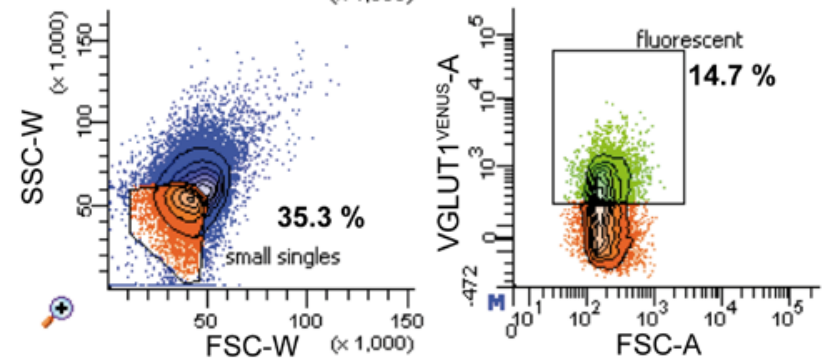

C Reanalysis of sorted VE-B - All Particles
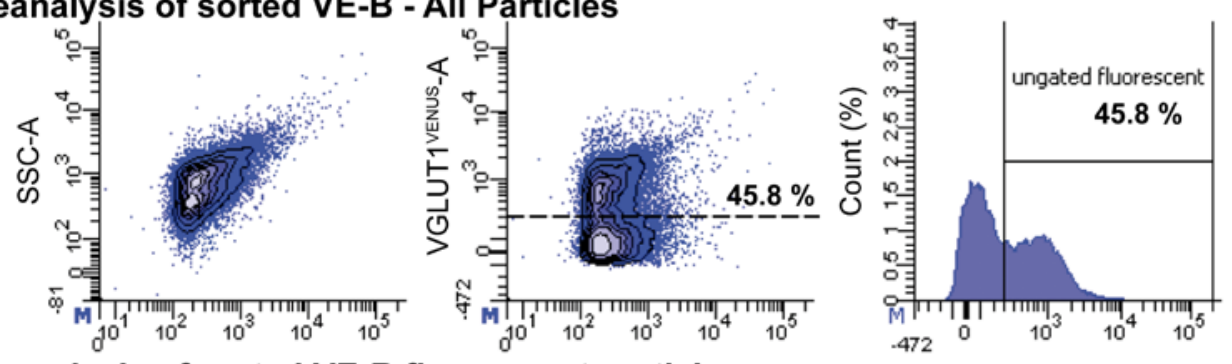

D Reanalysis of sorted VE-B fluorescent particles
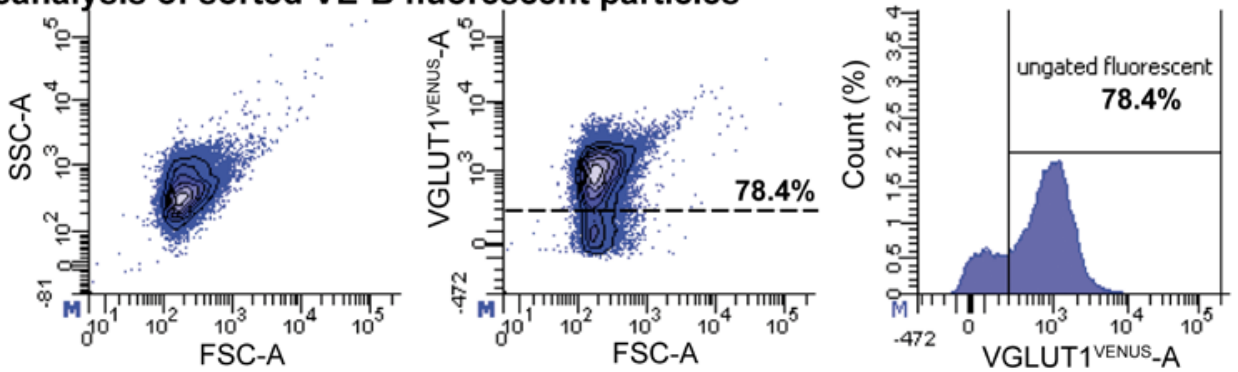


\section{Figure 11: FASS on gradient purified Synaptosomes (B)}

Flow analysis and sorting of gradient purified synaptosomes (B) of wild type and VGLUT1 ${ }^{\text {VENUS }}$ knock-in mice. (A) Analysis of wild type (top) and VGLUT1 ${ }^{\text {VENUS }}$ (bottom) synaptosomes before sorting. The left plots show the analysis of light scattering in forward angles (FSC) and at $90^{\circ}$ angle (SSC), which represent the relative size and internal complexity of the particles, respectively. The central plots display the relative size (FSC) and the fluorescence intensity in the VENUS channel. On the right the fluorescence intensity distribution of wild type and VGLUT1 ${ }^{\text {VENUS }}$ B particles is represented in a histogram. (B) Gating strategy to select preferentially single particles above wild-type background fluorescence. In order to preferentially select single events a gate was drawn to include particles of small SSC-width and FSC-width. Next the particles in this gate were analyzed for their fluorescence in the VENUS channel and a gate was drawn to include particles above wild-type background fluorescence. (C) Flow cytometric re-analysis of sorted P2 particles from VGLUT1 ${ }^{\text {VENUS }}$ mice. In this experiment all P2 particles were sorted and reanalyzed. (D) Flow cytometric re-analysis of sorted, fluorescent P2 particles from VGLUT1 ${ }^{\text {VENUS }}$ mice. In this experiment particles, which fell into the fluorescent gate (see VE-B in (B)), were sorted and re-analyzed.

\subsection{Aggregates within the B-fraction can be reduced by passage through the FACSAria and re-aggregation can be prevented using Pluronic F-68}

As illustrated by the comparison of $\mathrm{P} 2$ and $\mathrm{B}$ in the previous paragraph, the fraction of positive particles in the starting material had a considerable effect on sort speed and sample purity. In addition, when comparing sorted with unsorted synaptosomes, there was a reduction in the average size of particles after sorting. This can be seen by comparing the light-scatter plots (FSC-A/SSC-A in Figure $11 \mathrm{~A}$ and $\mathrm{C}$ ) and indicates that the sample may contain aggregates that are broken down by passage through the sorter. An alternative explanation for the reduction in average particle size would be the destruction of the particles themselves. For the example of a VGLUT1 ${ }^{\text {VENUS }}$ synaptosome one would expect this to result in a release of its vesicular content, with concomitant loss of VGLUT1 ${ }^{\text {VENUS }}$ fluorescence. This however seems unlikely, considering the presence of $45.8 \%$ of fluorescently positive particles in the sorted sample (Figure $11 \mathrm{C}$ ). 
A

wildtype-B

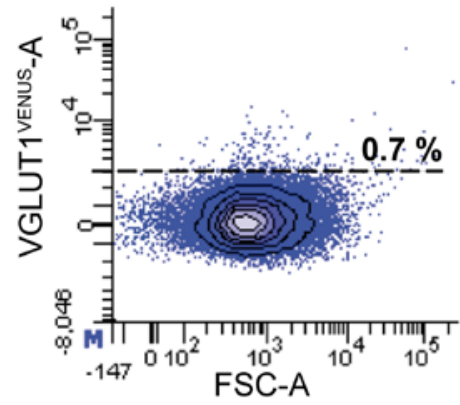

\section{VGLUT1 ${ }^{\text {VENUS }}$-B after $30 \mathrm{~min}$}

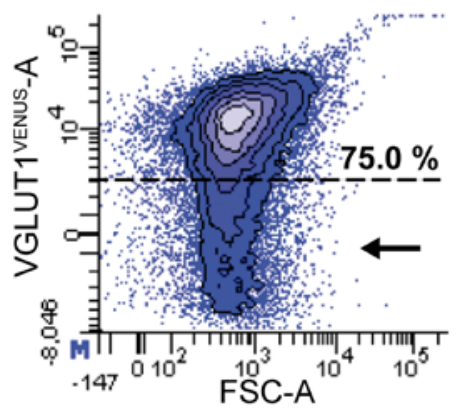

\section{VGLUT1 ${ }^{\text {VENUS }}$-B after $1 \mathrm{~h}$}
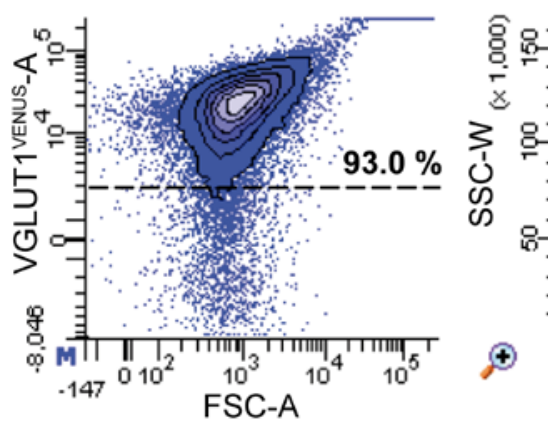
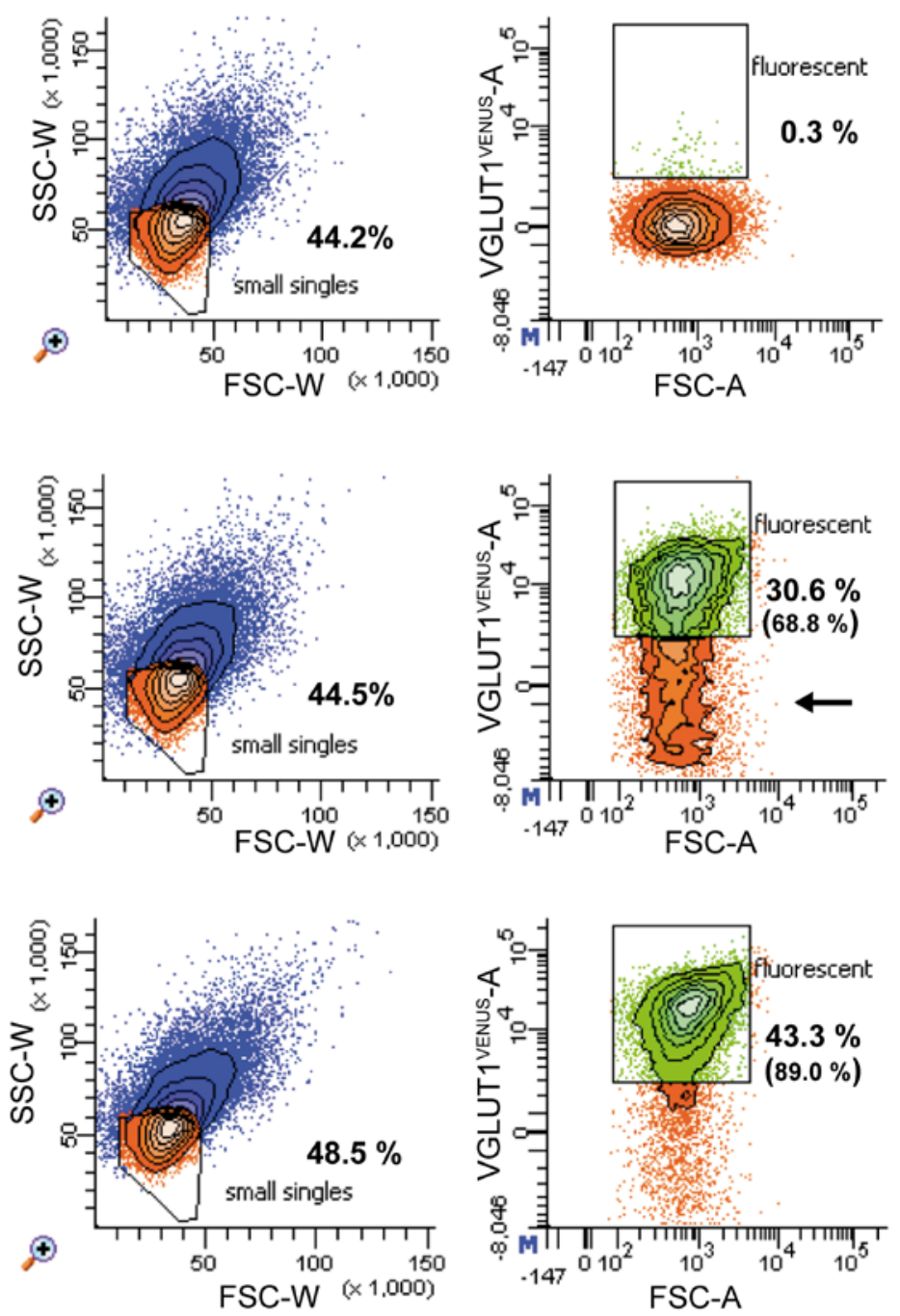

B

FACS broken VGLUT1 ${ }^{\text {VENUS }}$-B with $0.5 \%$ PF-68
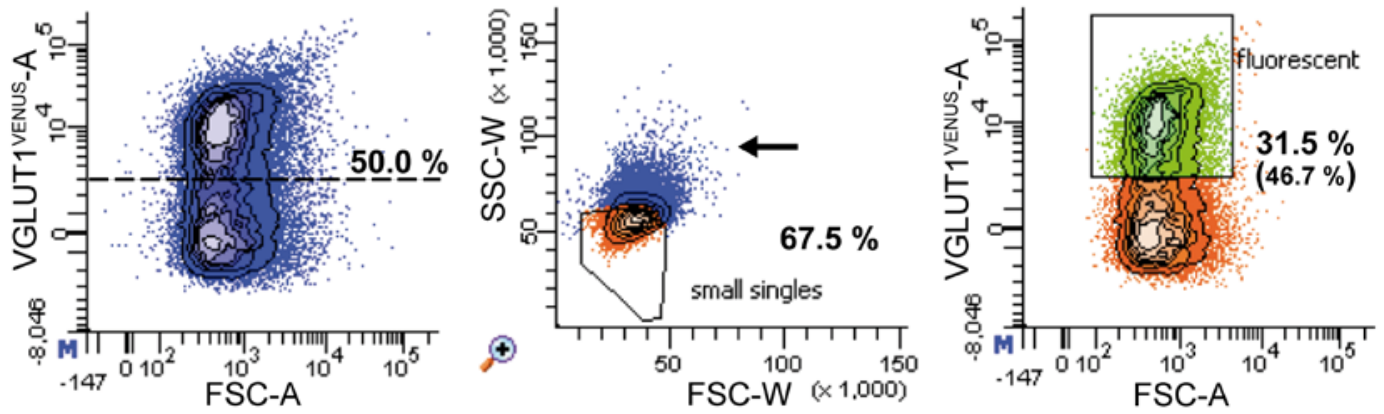

FACS broken VGLUT1 ${ }^{\text {VENUS }-B}$ with $0.5 \%$ PF-68 after $1 \mathrm{~h}$
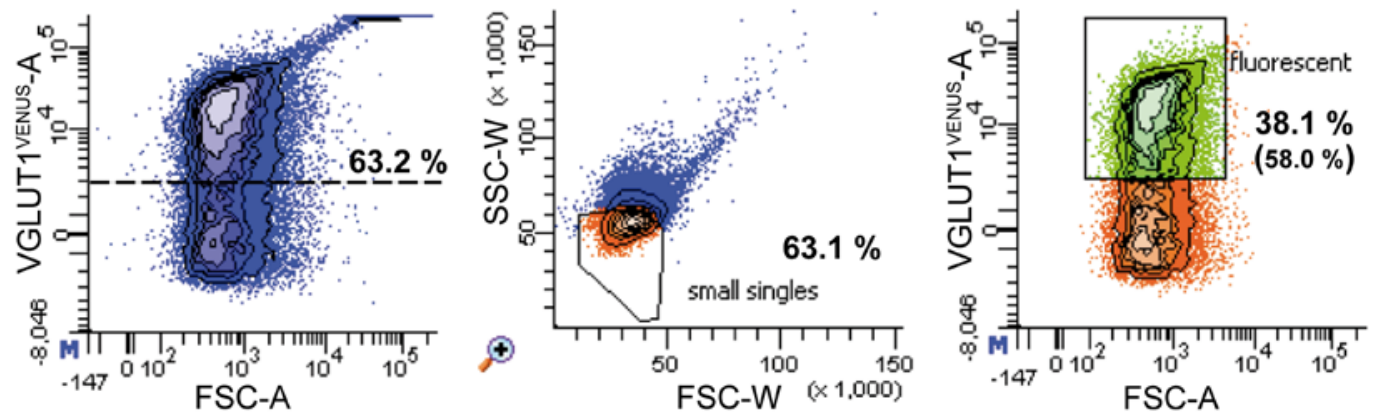


\section{Figure 12: Aggregate formation in the B-fraction during FASS was prevented by use of Pluronic F-68}

Description of the aggregate formation as well as strategies to reduce pre-existing aggregates and to prevent re-aggregation. (A) Gradient purified synaptosomes were diluted in FACS sample buffer and analyzed by flow-cytometry. Data were recorded at time point zero (see Figure 11), after $30 \mathrm{~min}$ in FACS sample buffer and after $1 \mathrm{~h}$ in FACS sample buffer. Note that the fraction of non-fluorescent particles (arrow) decreases over time. The wild type control was recorded at time 0 and serves to set the cutoff for fluorescence background. Values are the percentage of particles of the total population. Values in parentheses are percentages of particles of the "small single" population. (B) To break down pre-existing aggregates the sample was passed through the flow-cytometer in the presence of $0.5 \%$ Pluronic F-68 in the FACS sample buffer. Note the change in the distribution of SSC-width and FSC-width (arrow), as compared to unprocessed samples in (A) and in Figure 11. The FACS broken sample was also analyzed after $1 \mathrm{~h}$ in FACS sample buffer.

The relative contribution of the aggregates to the particles increases significantly over time (Figure $12 \mathrm{~A}$ ). One VGLUT1 ${ }^{\text {VENUS }}$ synaptosome will be enough to make the whole aggregate seem fluorescent. As a result, aggregation also increases the fraction of fluorescently positive particles (Figure $12 \mathrm{~A}$ ). Initially, the B-fraction contained 35.3 percent particles within the "small singles" gate. Of these particles, $41.8 \%$ (14.7\% of total) were detected as fluorescent. After $30 \mathrm{~min}$, the "small single" gate contains $44.5 \%$ of particles of which $68.8 \%$ (30.6\% of total) were fluorescent. After $1 \mathrm{~h}, 48.5 \%$ of particles were within in the "small singles" gate of which $89 \%$ (43.3\% of total) were fluorescent. This means that relative to time zero (Figure $11 \mathrm{~B}$ ) the fraction of small single particles that are also fluorescent increased by $110 \%$ after $1 \mathrm{~h}$. The aggregate formation prevents separation of fluorescent from non-fluorescent particles during longer sort experiments. In addition, these data indicate that the gating strategy employed to prevent analysis of aggregates by selecting events of low forward- and side-scatter width is not completely effective. In order to allow efficient purification of fluorescent VGLUT1 ${ }^{\text {VENUS }}$ synaptosomes also during long sorts, it was necessary to develop protocols that reduce the number of pre-existing aggregates in the sample and that prevent re-aggregation.

Already during analysis and re-analysis it was observed that processing a sample with the FACSAria would reduce the number of aggregates (section 3.1). Moreover, I had observed that addition of $0.5 \%$ Pluronic PF-68 to the FACS sample buffer was effective in preventing the aggregation of the B-fraction particles during the sort experiment. Therefore, I pre-processed the sample by passing a high concentration of particles through the FACSAria in the presence of $0.5 \%$ Pluronic PF-68, without sorting (section 2.4). This "FACS broken" sample contained $67.4 \%$ of particles within in the "small singles" gate of which $46.7 \%$ (31.5 $\%$ of total) were fluorescent. The analysis of the FACS broken sample after one hour showed that $63.1 \%$ of particles were within the "small singles" gate. Of these $58 \%$ (38.1\% of total) were fluorescent. This corresponds to a relative increase by $24 \%$ after one hour. The preprocessing through the FACS therefore resulted in a two-fold increase in single fluorescent 
synaptosomes available for sorting. However, the fraction of "small single" particles that was fluorescent was very similar to the unprocessed B fraction (46.7 \% vs. $42 \%$, Figure 11 and Figure 12). Furthermore, the addition of $0.5 \%$ Pluronic PF-68 to the FACS sample buffer slowed down aggregation by a factor of 4.6 .

\subsection{Sample recovery for Western blotting, electron microscopy and immunofluorescence microscopy after FASS}

The isolation of specific subpopulations of synaptosomes by FASS aims at analyzing the biochemical composition of these specific synaptosomes by methods such as Western blotting, proteomics and immunofluorescence microscopy. Moreover, it is necessary to demonstrate the integrity of the synaptic particles after FASS using electron microscopy (EM). All of these methods require specific sample preparations, which had to be developed and optimized.

\subsubsection{Vacuum filtration on polycarbonate filters is efficient in recovering synaptosomes for Western blotting and electron microscopy}

During fluorescence activated sorting of synaptosomes, the particle solution is diluted by several orders of magnitude. In case of the FACSAria, set up with the $70 \mu \mathrm{m}$ nozzle and sorting in "purity mode", the average particle is sorted in a volume of roughly $2 \mathrm{~nL}$ of buffer. This corresponds to $2 \mathrm{~mL}$ per million particles. Wolf et al. have estimated the protein content of 3 million sorted rat striatal synaptosomes to be in the range of $0.5 \mu \mathrm{g}$ (Wolf and Kapatos, 1989a). Considering these estimates I was aiming at the isolation of $50-70$ million particles in order to isolate 10 microgram of material. This would result in 100 to $140 \mathrm{~mL}$ of sorted sample, which have to be concentrated by a factor of 1000 in order to allow for analysis by Western blotting.

I therefore tested several strategies by concentrating a suspension of $10 \mu \mathrm{g}$ (total protein) of B-fraction in $100 \mathrm{~mL}$ FACS-Sheath fluid (PBS) and determining the recovery by 1D SDS PAGE and Coomassie staining (Summarized in Figure $13 \mathrm{~A}$ ). With a recovery $56 \%$ of protein, vacuum filtration through a $0.22 \mu \mathrm{m}$ polycarbonate filter (Isopore ${ }^{\mathrm{TM}}$, Millipore) proved to be the most effective method for recovering the diluted synaptosomes. The sorting of 50-70 million VGLUT1 ${ }^{\text {VENUS }}$ synaptosomes takes several hours. In addition, the filtration of $10 \mu \mathrm{g}$ (protein) of synaptosome takes 2-3 hours and requires the sorted material to be collected in a large container (100-140 mL). Plastic and glass surfaces can adsorb proteins and lipids unspecifically (Norde, 1992; Suelter and DeLuca, 1983). Therefore, also the sorted synaptosomes will be partly adsorbed to the surface of the collection device. I collaborated with the fine-mechanics workshop of the MPI of Experimental Medicine to design a collection 
device that allows the vacuum filtration to occur in parallel to the sorting. We produced a water cooled, online vacuum-filtration device designed to fit the FACSAria, which allows the collection and vacuum filtration of 2 samples simultaneously (Figure 13A and Appendix 2). In this way, the total time of the procedure, as well as the surface of the collection device were greatly reduced at the same time.

The Isopore ${ }^{\mathrm{TM}}$ filters were originally designed for the analysis of airborne contaminations by electron and light microscopy (Millipore homepage, http://www.millipore.com/catalogue/module/c153, 11.08.2010). Therefore, this method of particle concentration was also used to study the sorted material by EM (see 2.4.4 and 3.6.10) 
A

\begin{tabular}{|l|c|c|}
\multicolumn{1}{c|}{ Method } & Comment & Protein Recovery (\%) \\
\hline Reverse Osmosis (PEG, Dializorb) & failed & - \\
\hline Centriprep-30 & too slow, failed & - \\
\hline Centricon 70, MWCO8,000 & several hours & 13 \\
\hline Ultracentrifugation & $2-3$ hours & 16 \\
\hline Filtration NC $0.45 \mu \mathrm{m}$ & fast & 0 \\
\hline Filtration PC $0.1 \mu \mathrm{m}$ & too slow, failed & - \\
\hline Filtration PC $\mathbf{0 . 2 2} \mu \mathrm{m}$ & $\mathbf{2 - 3}$ hours & $\mathbf{5 6}$ \\
\hline Filtration PC $0.45 \mu \mathrm{m}$ & fast & 0 \\
\hline
\end{tabular}

B

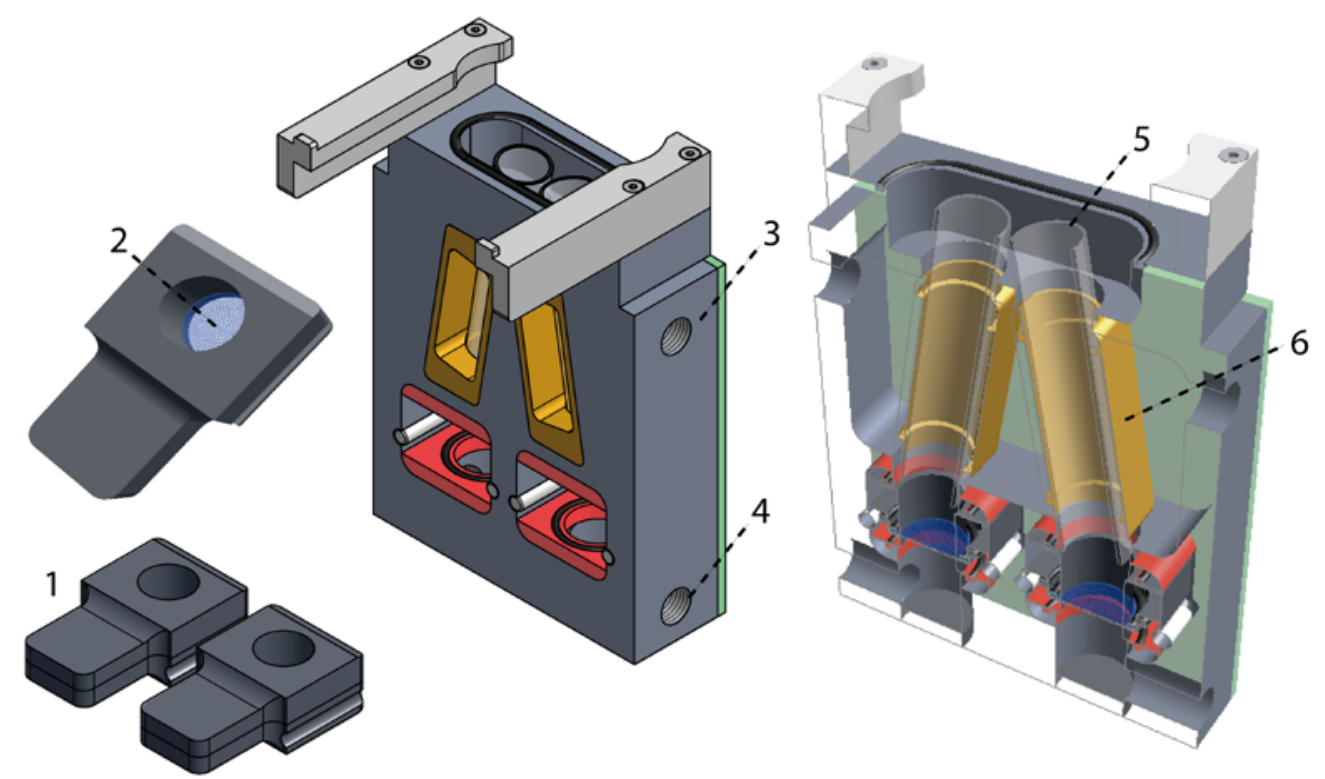

C
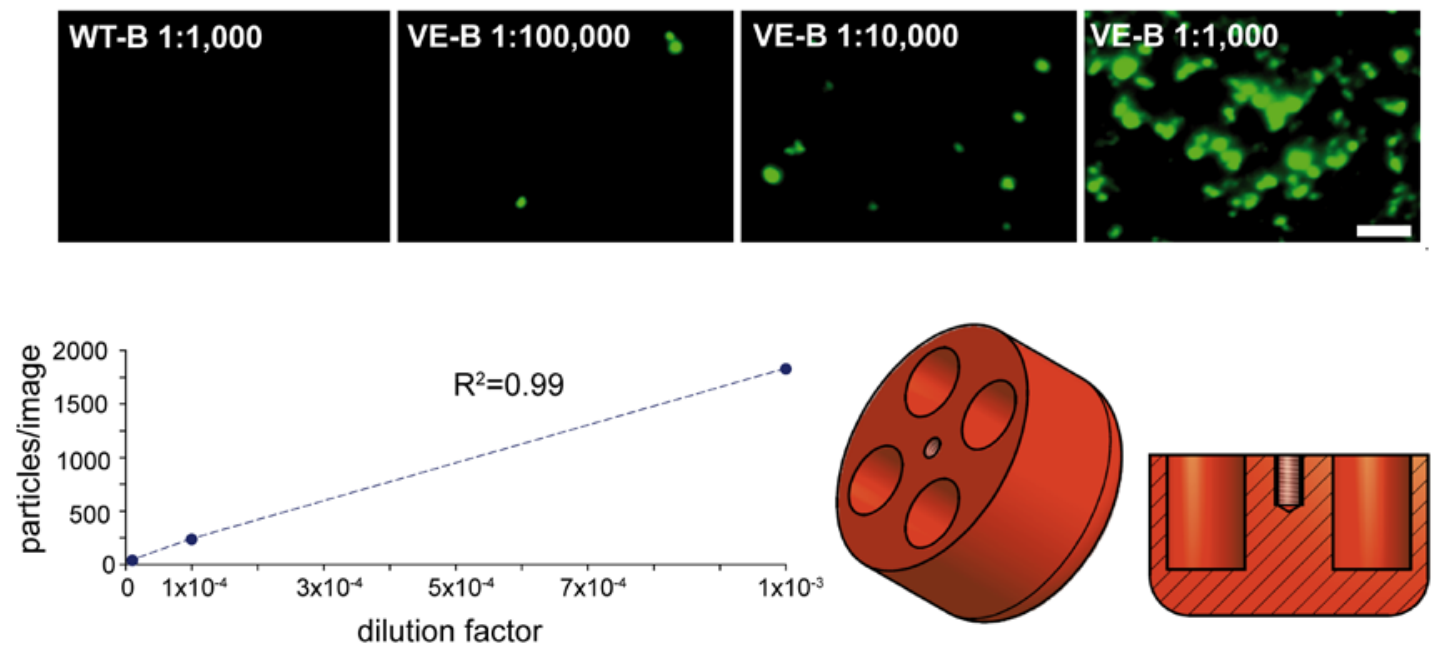

\section{Figure 13: Sample recovery after FASS}

Several methodological developments were made to allow the efficient analysis of FASS samples. (A) Different strategies were tested to recover synaptosomal proteins from $100 \mathrm{~mL}$ of a diluted synaptosome suspension. The dilution used was similar to the dilution introduced by FASS. Filtration using $0.22 \mu \mathrm{m}$ filters gave optimal results. (B) A custom-made vacuumfiltration device was designed to fit the FACSAria. During FASS, samples can be collected into either of two collection chambers. The wall of the collection chambers is lined by replaceable plastic vials, whose bottom was cut off and which can be replaced to reduce the 
risk of cross-contamination between samples (5). The device can be attached to a circulating water cooling system (3) and a metal casing around the collection chamber ensures efficient cooling of the collected sample (6). The bottom of the collection chamber consists of a removable filter cassette (1). The filter cassettes can be taken apart and an Isopore ${ }^{\mathrm{TM}}$ filter can be placed on top of a perforated plastic disc (2). This perforated plastic disc supports the Isopore ${ }^{\mathrm{TM}}$ filter during the vacuum filtration. To drive the filtration, vacuum is applied below the filter cassette holder (4); a waste bottle connected in between the vacuum source and the filtration device collects the filtrate. After the sample has been filtered, the cassette holder can be removed and the filter can be processed for subsequent analyses. (C) Recovery of VGLUT1 $^{\text {VENUS }}$ synaptosomes after FASS for immunofluorescence staining. Up to $4 \mathrm{~mL}$ (roughly $2 \times 10^{6}$ particles) of FASS sample can be centrifuged onto glass coverslips at up to $10.000 \times$ in custom-made centrifuge adaptors for the Beckman JA7.2 swing-out rotor. Using serial dilutions of synaptosomes in PBS, a quantitative immunostaining of VGLUT1 VENUS synaptosomes using a GFP antibody revealed a linear recovery of particles even from heavily diluted synaptosome suspensions.

\subsubsection{Centrifugation in custom adaptors allows quantitative recovery of diluted synaptosomes for immunofluorescence microscopy}

Even though the Isopore ${ }^{T M}$ filters were originally designed for microscopy, fluorescence background prevented their effective use for the study of sorted particles by immunofluorescence microscopy (data not shown). Therefore alternative strategies were employed.

In order to quantitatively pellet all particles of a diluted synaptosome suspension onto gelatinized glass coverslips I collaborated with the MPI of Experimental Medicine fine mechanics workshop to design centrifuge adaptors that allow high-speed centrifugation. The adaptors were manufactured to hold four coverslips and fit into the Beckman JA 7.5 swingout bucket rotor. For each coverslip a volume of $4 \mathrm{~mL}$ could be centrifuged. The JA 7.5 rotor has four buckets and therefore allows the simultaneous centrifugation of four adaptors holding a total of 16 coverslips at a maximum speed of 7,500 rpm (10,500 x g). The adaptors were manufactured from Delrin ${ }^{\circledR}$, which is suitable for centrifugation and resistant to salt, aldehydes and alcohol.

(www.k-mac-plastics.net/data\%20sheets/delrin chemical resistant chart.htm, 11.08.2010)

In order to test the quantitative recovery of synaptosomes from diluted suspensions, I made serial dilutions of a B-fraction with dilution factors of 1:1,000, 1:10,000 and 1:100,000. $3 \mathrm{~mL}$ of diluted synaptosomes were centrifuged at $10,500 \times \mathrm{g}$ for 20 min onto gelatinized glass coverslips. Subsequently, particles were stained for VGLUT1 ${ }^{\text {VENUS }}$ using an anti-GFP antibody and epifluorescence images were quantified for number of VGLUT1 ${ }^{\text {VENUS }}$ positive particles per field (Figure 13C). Linear recovery of particles from different dilutions indicated that a representative fraction of particles was pelleted onto the coverslips. Additionally, when processing sorted synaptosomes for immunofluorescence microscopy by this method, the supernatant of the centrifugation was devoid of particles as analyzed by flow cytometry 
(FACSAria, data not shown). This means that all particles that were detected by flow cytometry were quantitatively removed from the solution using this centrifugation protocol.

\subsection{FASS in FSC-mode does not remove inhibitory synaptosomes (VIAAT) or myelin (PLP)}

Generally the FACSAria is set up to decide whether an incoming signal corresponds to a particle based on its light scattering properties in the FSC Channel. In order to separate real events from background scattering from the buffer or electronic noise, one introduces a threshold on the FSC channel, which masks such events (FSC-mode). Sorting of the VGLUT1 ${ }^{\text {VENUS }}$ fluorescent population in FSC-mode strongly enriched fluorescent particles as analyzed by flow cytometry (Figure 11D and Figure 14B). In order to investigate whether the sorted particles indeed correspond to VGLUT1 ${ }^{\text {VENUS }}$ synaptosomes, I sorted 50 million VGLUT1 $^{\text {VENUS }}$ positive particles using the FACSbreak/pluronic procedure (Figure $12 \mathrm{~B}$ ). The sorted particles were recovered by vacuum filtration and then analyzed and compared with the starting material by Western blotting. FASS in FSC-mode resulted in an enrichment of VGLUT1 ${ }^{\text {VENUS }}$ immunoreactivity as assessed in Western blotting. However, also VIAAT immunoreactivity and PLP immunoreactivity were strongly increased in the sorted fraction (Figure 14 C). VIAAT specifically labels inhibitory synaptic vesicles and synaptosomes, while PLP is one of the most abundant proteins in myelin. Both of these proteins should have been depleted, if the FASS strategy that I employed actually purified single VGLUT1 ${ }^{\text {VENUS }}$ synaptosomes.

Other groups have reported synaptosomes to be in the range of $0.75-1.5 \mu \mathrm{m}$ polystyrene beads in terms of forward angle and $90^{\circ}$ light-scattering (Wolf and Kapatos, 1989b). In the present study the B-fraction particles analyzed in FSC-mode were also detected in a similar range (Figure $14 \mathrm{D}$ ). In addition, I was selecting the particles at the lower end of the size spectrum in my preparation (Figure $11 \mathrm{D})$. However, considering the Western blot results, I concluded that despite the efforts to reduce and prevent aggregation and the agreement in size with previous publications, I was still isolating aggregates. This implies that the single synaptosomes are smaller than the particles I had isolated so far and should therefore be expected at lower FSC-A values. 
A

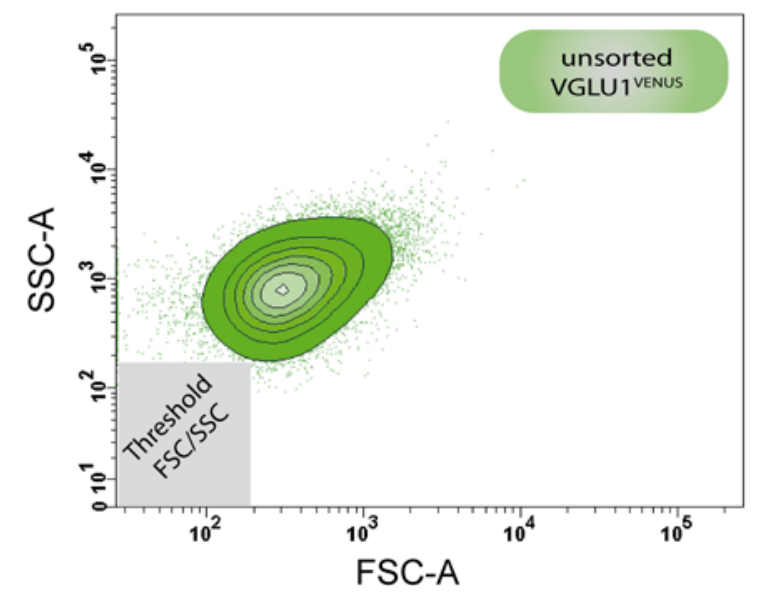

C

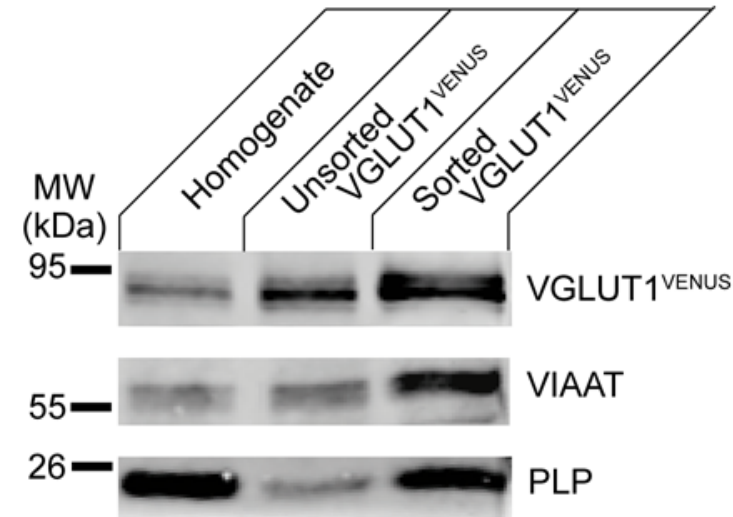

B

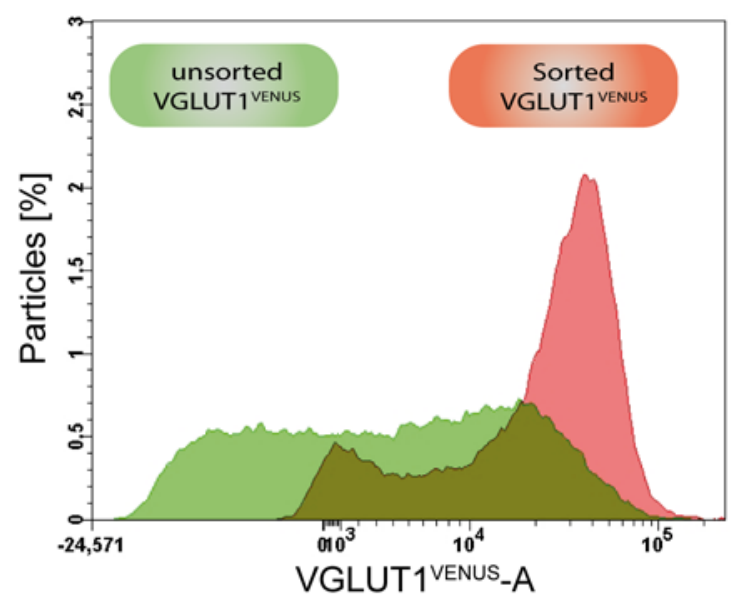

D

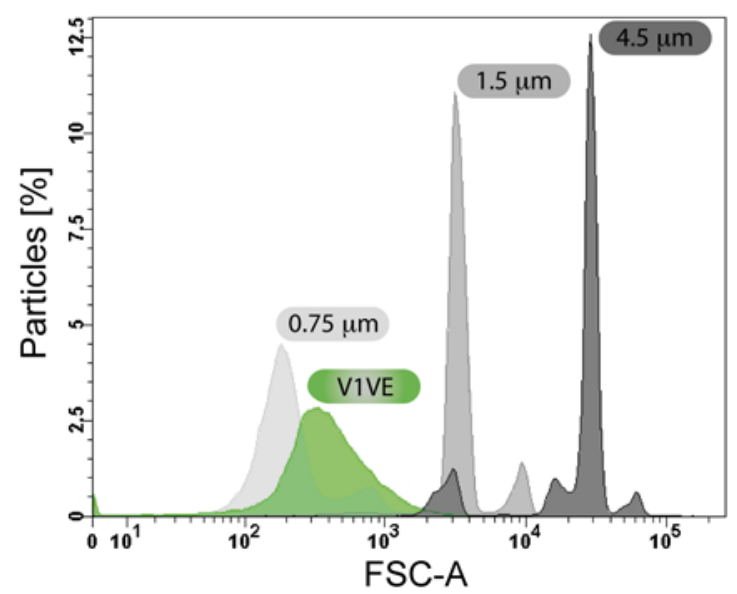

Figure 14: FASS in FSC mode does not deplete VIAAT or PLP

Results of a bulk isolation of VGLUT1 ${ }^{\text {VENUS }}$ using the FACSAria in FSC-mode show the coenrichment of VIAAT and PLP. (A) Analysis of light-scattering distribution of unsorted VGLUT1 $1^{\text {VENUS }}$ synaptosomes. The instrument threshold in the FSC channel is indicated. (B) Histogram showing the distribution of VGLUT1 ${ }^{\text {VENUS }}$ fluorescence in unsorted VGLUT1 ${ }^{\text {VENUS }}$ and sorted fluorescent VGLUT1 ${ }^{\text {VENUS }}$. Note the strong enrichment in fluorescently intense particles in the sorted sample. (C) Western blot analysis of homogenate, unsorted VGLUT1 $1^{\text {VENUS }}$ synaptosomes and sorted VGLUT1 ${ }^{\text {VENUS }}$ positive particles. Note the strong reenrichment of PLP. (D) Analysis of the light scattering properties of unsorted synaptosomal preparations from VGLUT1 ${ }^{\text {VENUS }}$ knock-in mice in relation of polystyrene microspheres of known diameter. Note that the particles of the synaptosomal preparation are in between 0.75 and $1.5 \mu \mathrm{m}$ beads.

\subsection{Membrane staining with FM4-64 is compatible with VGLUT1 ${ }^{\text {VENUS }}$ detection and reveals synaptosomes in a lower size range}

The enrichment of VIAAT and PLP in VGLUT1 ${ }^{\text {VENUS }}$ positive particles isolated by FASS in FSC-mode as assessed by Western blot analysis indicated that the isolated particles were aggregates (see previous paragraph). Therefore single synaptosomes should 
be smaller and found at lower FSC-A values. The particles isolated were already at the lower end of the FSC-A detection range. When lowering the threshold for detection in FSC further one starts to also detect the background signal, which is generated by the sheath fluid (PBS) and electronic noise. When the threshold is lowered to a minimum (no threshold) the buffer generates a background signal that is detected at a rate of $2000 \mathrm{evt} / \mathrm{s}$ (Figure $15 \mathrm{~A}$ ). At event rates of 20,000-25,000 evt/s during high-speed sort experiments this background would decrease sort speeds by at least $10 \%$. Additionally, during re-analysis of the very diluted sorted fractions, with event rates in the range of 100-300 evt/s, the background signal would contaminate the corresponding data significantly and not allow to record reliable data.

Instead of triggering on light-scatter signals one can also set the instrument to trigger on any one of the fluorescent channels. I therefore included a membrane staining using the lipophilic styryl dye FM4-64 into my protocol. Due to its lipophilic properties FM4-64 will label all membranous particles in the B-fraction. FM4-64 is hardly fluorescent in aqueous solutions, but becomes strongly fluorescent when partitioning into membranes (Gaffield and Betz, 2006; Vida and Emr, 1995). FM4-64 can be excited using the $488 \mathrm{~nm}$ laser of the FACSAria. In order to characterize its fluorescence properties in our preparations we performed particle based $\lambda$-scans using a Leica-SP5 confocal microscope (analysis performed by EH). FM4-64 showed a broad emission peak between $560 \mathrm{~nm}$ and $800 \mathrm{~nm}$ when incorporated into the membranes of particles in the B-fraction (Figure $15 \mathrm{CD}$ ). When analyzing the emission spectrum of wild type particles stained with FM4-64, there was no signal in the detection window of VGLUT1 ${ }^{\text {VENUS }}$ (Figure 15 D) Therefore, FM4-64 staining is compatible with detection of VGLUT1 ${ }^{\text {VENUS }}$ without spectral overlap when using a 530/30 BP. filter for detection of the VGLUT1 ${ }^{\text {VENUS }}$ fluorescence.

The FACSAria was then set up to trigger on the signal in the FM4-64 channel (488 nm laser excitation, 695/40BP-filter for detection). When recording just the FACS-sample buffer containing FM4-64 at the working concentration of $1.5 \mu \mathrm{g} / \mathrm{mL}$ without threshold, one detects the buffer background in the FM4-64 channel (Figure $15 \mathrm{~B}$ ). Carefully setting the threshold in the FM4-64 channel successfully removed the buffer background from the analysis of FM-4-64 stained synaptosomes. Interestingly, analysis of the FSC-A/SSC-A properties revealed that $72.9 \%$ of the FM4-64 stained particles fall within the region that was previously masked by thresholding on the FSC channel (Figure $15 \mathrm{~B}$, Figure 14 A). The smaller size of the particles stained by FM4-64 is also illustrated when measuring this population in relation to different size polystyrene microspheres. The vast majority of the particles were detected at FSC-A values below the signal of the $0.75 \mu \mathrm{m}$ polystyrene beads and therefore smaller than the previously isolated particles (compare Figure $14 \mathrm{D}$, Figure 15 B). 
A

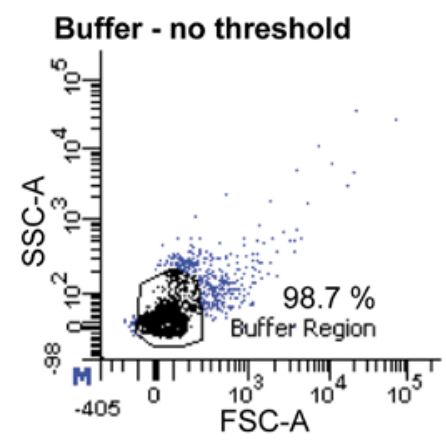

Synaptosomes - FSC-mode

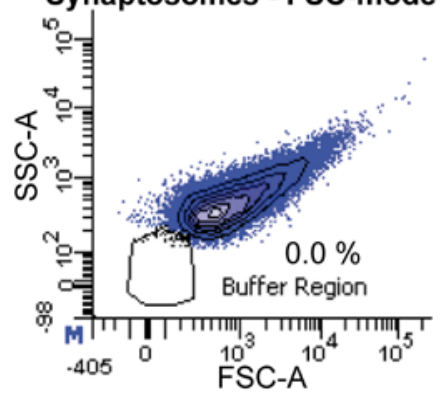

B

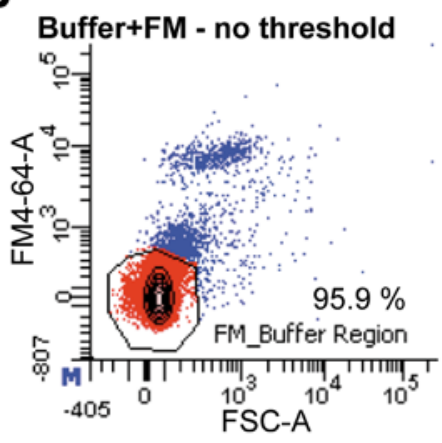

Synaptosomes - FM-mode

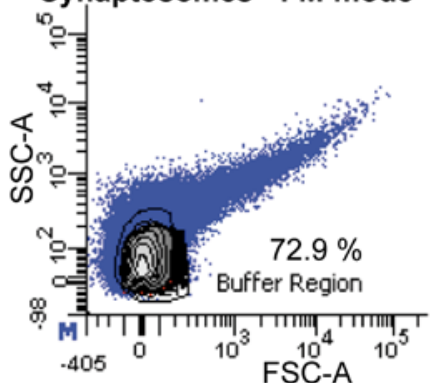

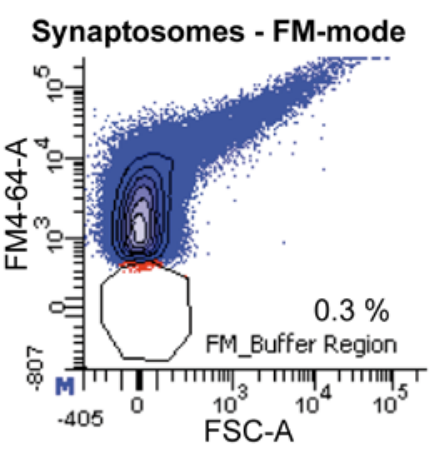

Particle Size - FM-mode

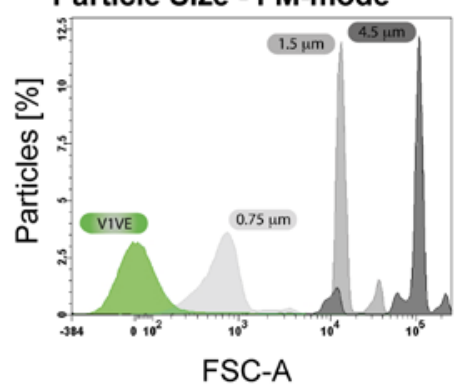

\section{C}
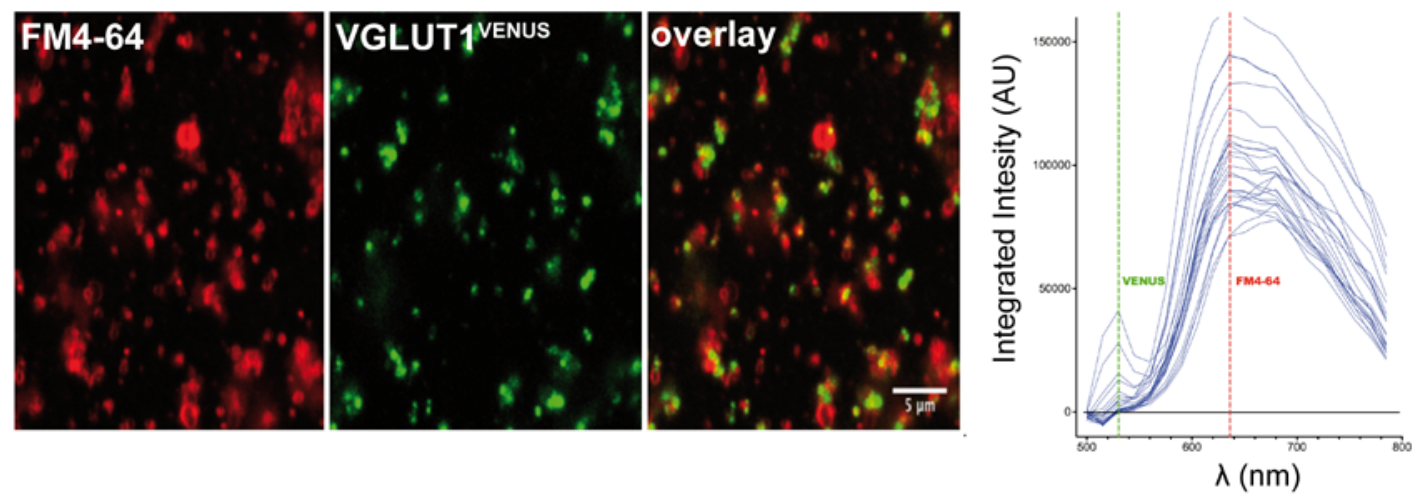

D
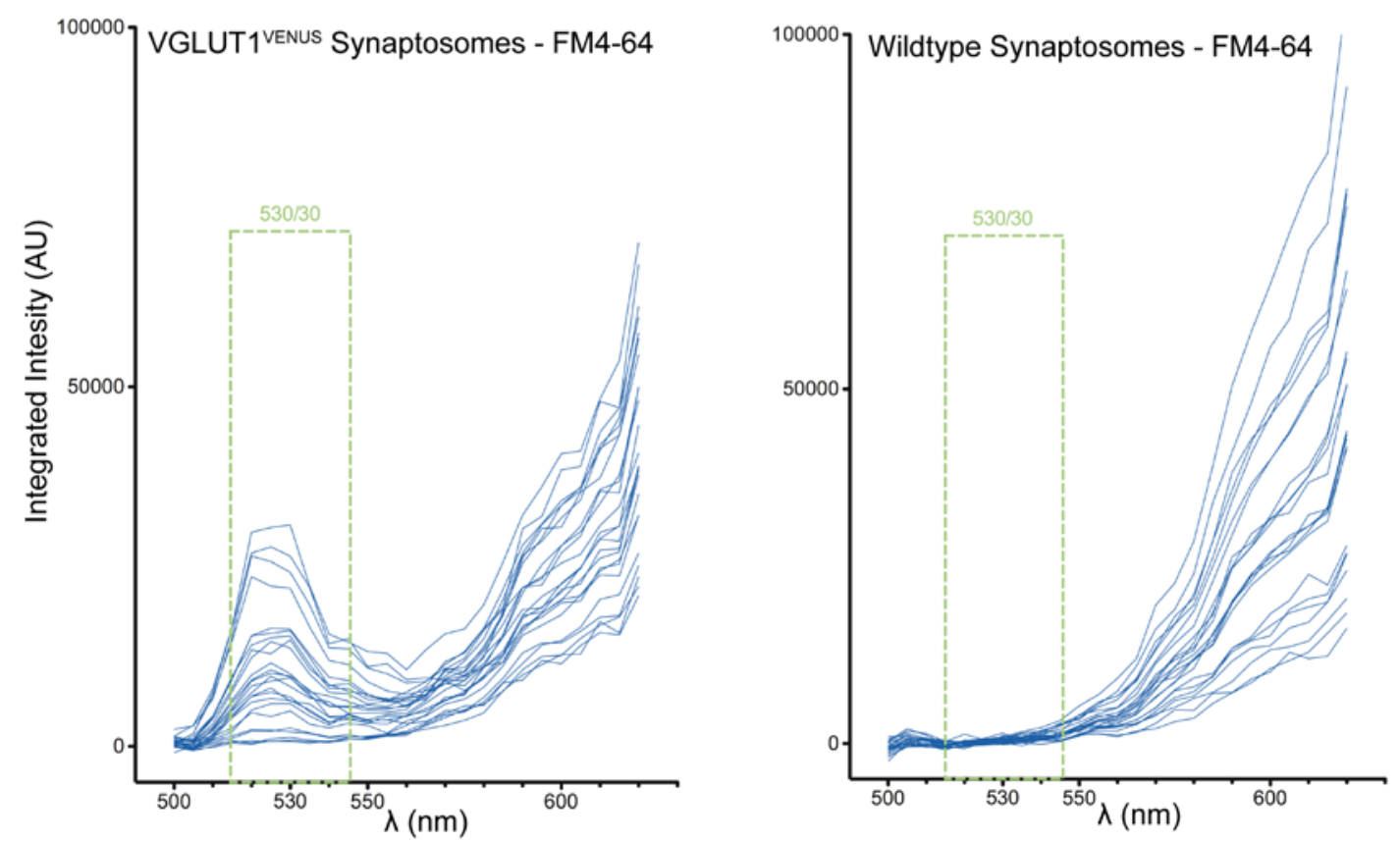


\section{Figure 15: FM4-64 reveals B-fraction particles at smaller size}

Analysis of synaptosomes stained with the lipophilic styryl dye FM4-64 in order to allow detection of small, membrane containing particles for FASS. (A) (top) Analysis of the lightscattering of FACS sample buffer, without any threshold for particle/background discrimination. This illustrates the level of buffer background and noise. (bottom) Analysis of light scattering of synaptosomes with a threshold on the FSC to block out the background from the sample analysis. Note the Buffer region gate contains $0 \%$ of particles. (B) (top-left) Analysis of the FM4-64 fluorescence and FSC characteristics of FACS sample buffer containing FM-dye, without any threshold for particle/background discrimination indicating the level of FM-buffer background. (top-right) Analysis of the FM4-64 fluorescence and FSC of synaptosomes stained with FM4-64 with a threshold on the FM4-64 channel to block out background from the sample analysis. (bottom-left) Analysis of the light-scattering of FM4-64 stained synaptosomes analyzed in FM-mode. Note that the light-scatter Buffer_region contains $72.9 \%$ of particles (compare to A) (bottom-right) Analysis of the light-scattering of the FM4-64 stained synaptosomes analyzed in FM-mode in relation to different size polystyrene microspheres. Note that the FM-mode population shows light scattering below the level of $0.75 \mu \mathrm{m}$ beads. (C) Particle based $\lambda$ scan of FM4-64 stained VGLUT1 ${ }^{\text {VENUS }}$ synaptosomes using a confocal microscope. Using laser-excitation at a wavelength of 488 $\mathrm{nm}$, the emission spectrum of several particles was recorded between $500 \mathrm{~nm}$ and $800 \mathrm{~nm}$ using a window size of $10 \mathrm{~nm}$. Note that all particles recorded are fluorescent for FM4-64, while there are differences in the level of VGLUT1 ${ }^{\text {VENUS }}$ fluorescence. (D) Comparison of $\lambda$ scans of wild type and VGLUT1 ${ }^{\text {VENUS }}$ synaptosomes stained with FM4-64. The bandwidth of the detection filter for VGLUT1 $1^{\text {VENUS }}$ used on the FACSAria is indicated. Note that in wild type synaptosomes stained with FM4-64 no fluorescence is detected in this window.

The data from membrane staining with FM4-64 allow to further interpret the Western blot results of FASS in FSC-mode. Fluorescence activated sorting does not sort the particles directly, but instead sorts 1-2 drops of liquid, which contain the particle of interest. If unwanted particles are detected in these drops they will not be sorted. However, as illustrated above the vast majority of particles in the B-fraction were below the threshold of detection in FSC-mode. This means that for every fluorescent particle sorted in FSC-mode the sorted drops also contained several subthreshold particles. Since these subthreshold particles can be stained with FM4-64 they contain biological membranes. This means that subthreshold also contain proteins and may have contributed significantly to the overall protein yield. Could the co-sorted subthreshold particles also have contributed to the observed enrichment in VIAAT and PLP? The subthreshold particles were co-sorted nonselectively. Accordingly, the contribution of different particle types to the sorted material will be proportional to their contribution to the B-fraction. Therefore co-sorting subthreshold particles should not contribute to the enrichment of any particular protein of the B-fraction. For this reason, the enrichment of VGLUT1 ${ }^{\text {VENUS }}$, VIAAT and the strong enrichment in PLP by FASS in FSC-mode reflects the composition of the sorted fluorescent particles. This strengthens the previous conclusion that the particles isolated in FSC-mode were aggregates. These myelin rich aggregates probably contain at least one VGLUT1 ${ }^{\text {VENUS }}$ synaptosome and also other types of synaptic particles such as VIAAT containing synaptosomes. Single synaptosomes are likely found in the previously masked FSC-A range 
and can be visualized by staining with FM4-64. Thus, FASS in FM-mode should allow enrichment of single VGLUT1 ${ }^{\text {VENUS }}$ synaptosomes.

\subsection{Analysis of FASS in FM-mode}

The introduction of FM4-64 staining and the change to triggering/thresholding in the FM4-64 channel during FASS represented the final step of method optimization. The final FASS procedure is illustrated in Figure 18, which also outlines the method validation by EM, immunofluorescence microscopy and Western blotting, as well as the use of FASS to screen for novel synaptic proteins in a proteomics screen which will be described in the following paragraphs.

\subsubsection{FASS in FM-mode enriches VGLUT1 ${ }^{\text {VENUS }}$ synaptosomes as analyzed by flow cytometry}

As described above, membrane staining with FM4-64 and measuring in FM-mode reveals a large number of particles in the B-fraction that are smaller than the particles previously isolated by FASS in FSC-mode. Less than $10 \%$ of the particles detected in FMmode were in the size range of the aggregates in FSC-mode (see "FSC_mode population"gate in Figure 16). When comparing wild type and VGLUT1 ${ }^{\text {VENUS }}$ synaptosome preparations, between 12 and $20 \%$ of particles are of small size and above wild-type background fluorescence. Interestingly, the difference between unsorted (V1VE) and FACS processed (V1VE-all particles) was much smaller than previously observed in FSC-mode (compare Figure 11 and Figure 16). The passage through the FACS reduced the fraction of particles in the "FSC_mode population" gate from $7.1 \%$ to $2.3 \%$. In addition, the fraction of "small singles" gate particles increased from $88.5 \%$ to $94.6 \%$ and the fraction of particles in the "fluorescent" gate increased from $12.5 \%$ to $16.0 \%$. These shifts in population percentages can be interpreted as the breakdown of aggregates present in the "FSC_mode population" gate. The aggregate breakdown during passage through the FACS would generate several smaller particles per broken aggregate and in this way increase the percentage of particles that fall within the "FM_mode small size gate" during re-analysis. The "fluorescent" gate is a sub-gate of the "Small Size" gate. Accordingly, the increase in the number of small particles also results in a greater fraction of all particles within the "fluorescent' gate.

Sorting the particles in the "fluorescent" gate resulted in an increase in ungated fluorescence from $23.3 \%$ to $52.3 \%$ (Figure 16). The fraction of particles within the "FSC_mode population" gate decreased from $7.1 \%$ to $0.5 \%$, while the fraction of particles in the "Small Size" gate increased from $88.5 \%$ to $97.2 \%$. At the same time, the percentage of particles falling into the fluorescent gate increased from $12.5 \%$ to $46.2 \%$. This corresponds 
to a 3-fold enrichment in small fluorescent particles. It can also be noted that the histograms of fluorescence intensity distributions of wild type and sorted VGLUT1 ${ }^{\text {VENUS }}$ are partially overlapping (Figure 16). Therefore, simply taking the wild-type background fluorescence level as a cutoff underestimates the fraction of VGLUT1 ${ }^{\text {VENUS }}$ positive particles in the sorted sample.
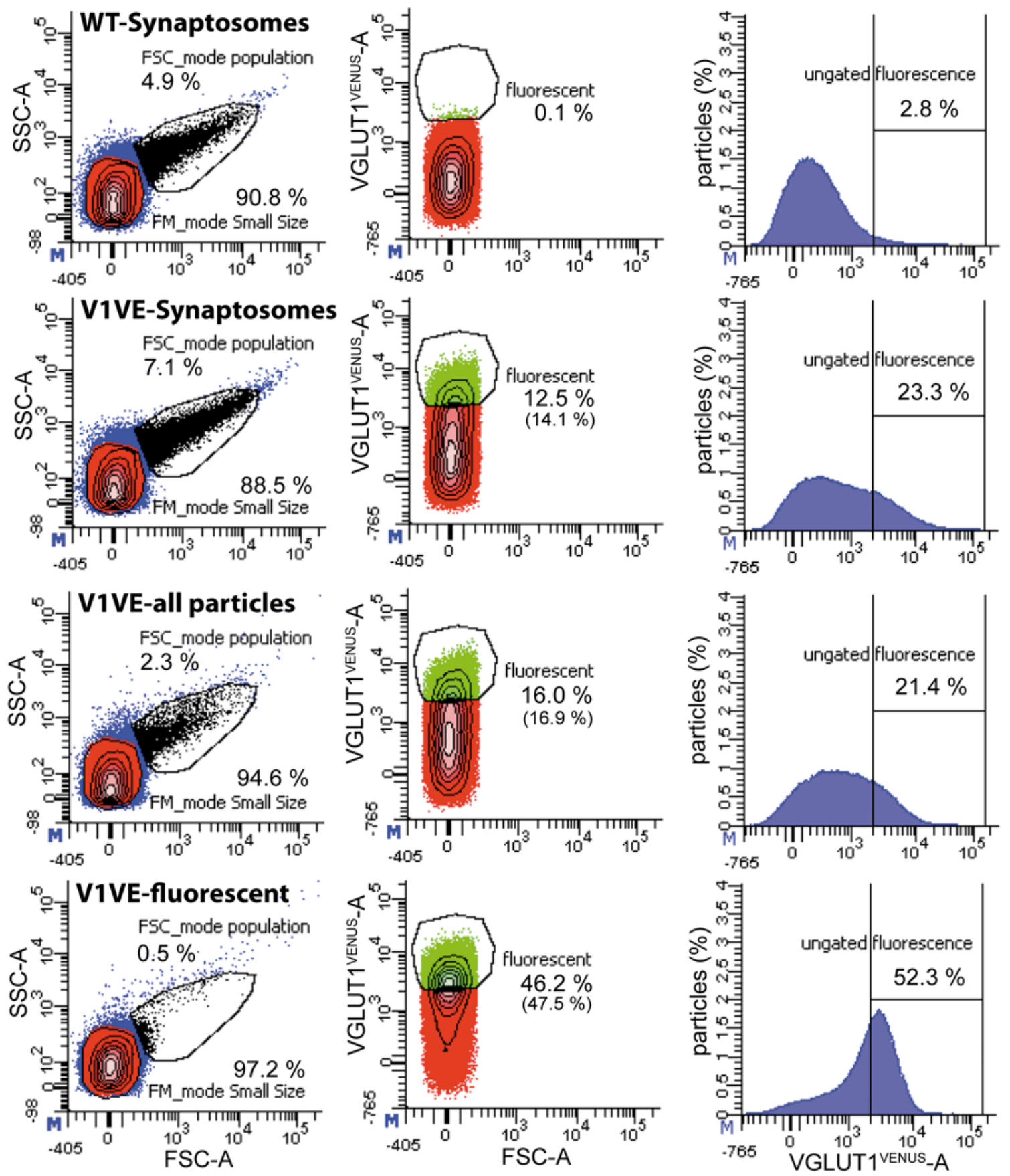


\section{Figure 16: FASS in FM-mode enriches VGLUT1 ${ }^{\text {VENUS }}$ positive particles}

Flow cytometric analysis of unsorted wild type and VGLUT1 ${ }^{\text {VENUS }}$ synaptosomes, as well as sorted VGLUT1 ${ }^{\text {VENUS }}$ particles in FM-mode. Left: This column shows analysis of light scattering. Light scattering was used to define two gates. The "FSC_mode population" gate marks particles that are in light-scattering ranges of the particles analyzed and sorted in FSC_mode (see Figure 15). The "FM_mode Small size" gate selects those particles that are smaller than the FSC mode population. Center: The events of the "FM mode Small size" gate are plotted according to VGLUT1 ${ }^{\text {VENUS }}$ fluorescence and forward angle light scatter (FSC). A gate was drawn to include particles that are above wt-fluorescence. The percentages in parentheses are referring to the "FM_mode Small size gate" population, while all other values refer to percent of all particles. Right: Histograms showing the distribution of VGLUT1 $1^{\text {VENUS }}$ fluorescence intensity of all particles in the different samples, a gate was drawn at the same fluorescence threshold as used for the "fluorescent" gate.

\subsubsection{Fitting of the flow-data with a multiple normal distribution component model predicts an approximate 8.8 -fold relative enrichment of VGLUT1 ${ }^{\text {VENUS }}$ synaptosomes compared to non-fluorescent particles}

During FASS a binary fluorescence threshold is used in order to separate VGLUT1 ${ }^{\text {VENUS }}$ synaptosomes from non-fluorescent particles. This fluorescence threshold was based on the background fluorescence of the wild type sample. When analyzing the VGLUT1 $1^{\text {VENUS }}$ synaptosomes for their fluorescence distribution, they do not show as two populations of particles (non-fluorescent and fluorescent). Instead, the particles show a continuous distribution of fluorescence intensities with $22 \%$ of particles above wild-type background fluorescence. After these particles are sorted and reanalyzed, their fluorescence signal has considerable overlap with the wild type distribution. Taking together, these results indicate that FACSAria is not sensitive enough to completely separate the non-fluorescent from the fluorescent particles in the synaptosomal preparation. In order to make a more precise estimation of the contribution of different particle populations to the unsorted and sorted synaptosomes, we fitted the FACS data with a multiple normal distributions model after certain data transformations. (described in the following paragraph, data analysis was performed by Dr. Liyi Li, Dept. of Molecular Neurobiology, MPI-EM).

The flow-cytometry data were exported from the FACSDiva software and were transformed according to the algorithm published by Parks et al. (2006) (see also 2.4.3). The transformed data of four wild type measurements (each 100,000 data points) were fitted with a multiple normal distribution model respectively. In all cases the optimal fitting required a minimum of three normal distribution components to sum up. With the parameters (proportion, mean, standard deviation) from these four independent fits, an average probability distribution function (PDF) of the wild type was calculated. This wild type-PDF (with the proportion as a free parameter) was then incorporated into another multiple normal distribution component model used to re-analyze the sorted VGLUT1 ${ }^{\text {VENUS }}$ synaptosomes. Apart from the wild type-PDF, a minimum of two normal distributions were required to achieve an optimal fitting of the data of sorted VGLUT1 ${ }^{\text {VENUS }}$-all particles and sorted 
VGLUT1 ${ }^{\text {VENUS }}$ fluorescent particles. This model therefore describes the data as a mixture of three normally distributed populations of particles:

o a non-fluorescent wild type-like population

o a population of intermediate fluorescence

0 a population of fluorescently positive particles.

In the "sorted all particles" sample, $25.9 \pm 5.6 \%$ of the particles were attributed to the wild type-like population, $56.4 \pm 5.1 \%$ to the intermediate population and $18.0 \pm 5.2 \%$ to the fluorescent population. In the "sorted fluorescent particles" $12.4 \pm 0.7 \%$ were counted to the wild type-like, $23.0 \pm 2.4 \%$ to the intermediate and $64.8 \pm 1.9 \%$ to the fluorescent population.

A binary threshold, for the separation of fluorescent particles on the basis of wild type fluorescence background is indicated in Figure 17. It is evident that a binary threshold set on the basis of wild-type background fluorescence selects a large number of fluorescent particles but will not be able to fully exclude contributions from the intermediate and wild type populations. In agreement with this interpretation the wild type and intermediate populations contribute also to the sorted fluorescent particles sample.

According to this analysis the binary threshold will therefore underestimate the fraction of fluorescently positive particles in the sorted fluorescent sample. However, the multiple normal distribution component model can give a precise estimation of the relative contributions of each particle population. It predicts that in the unsorted material, about $18 \%$ of particles are fluorescently positive VGLUT1 ${ }^{\text {VENUS }}$ synaptosomes, while in the sorted fraction about $65 \%$ of particles are fluorescently positive synaptosomes. This corresponds to a 3.6-fold enrichment of VGLUT1 ${ }^{\text {VENUS }}$ synaptosomes. In addition, one can also analyze the ratios of fluorescent to intermediate or non-fluorescent components. There, the unsorted sample shows a ratio of 0.69 (fluo/wt), 0.32 (fluo/intermediate) and 0.21 (fluo/rest). For the sorted samples the ratios change to 5.2 (fluo/wt), 2.8 (fluo/intermediate) and 1.84 (fluo/rest). Consequently, the relative enrichment of fluorescent particles to wild type-like particles between the unsorted and sorted sample would be 7.54-fold. The relative enrichment of fluorescent to intermediate particles would be 8.75 -fold and the relative enrichment of fluorescent particles to the rest of particles would be 8.76 fold. Therefore, while one can expect a 3.6-fold enrichment in the fraction of fluorescent particles, the relative enrichment of components of the VGLUT1 $1^{\text {VENUS }}$ synaptosomes compared to other particles in the preparation can be expected to be significantly higher for both non-fluorescent particles and for particles of intermediate fluorescence. 
A

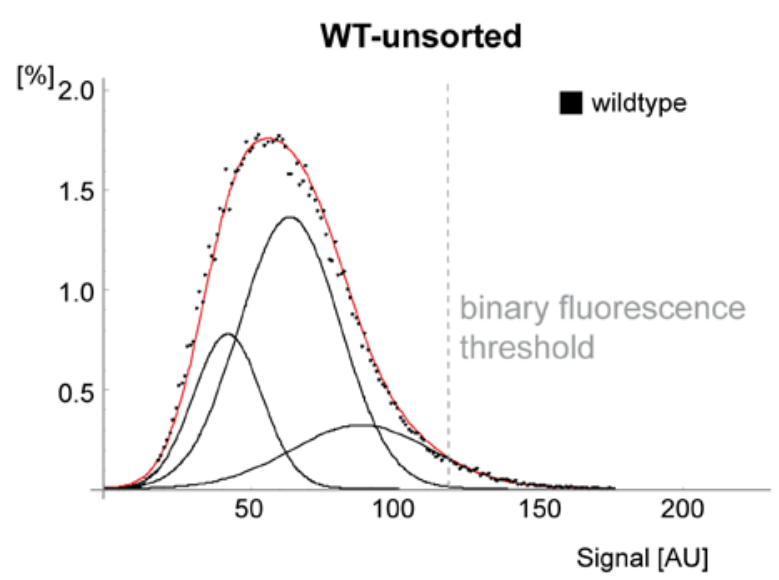

B
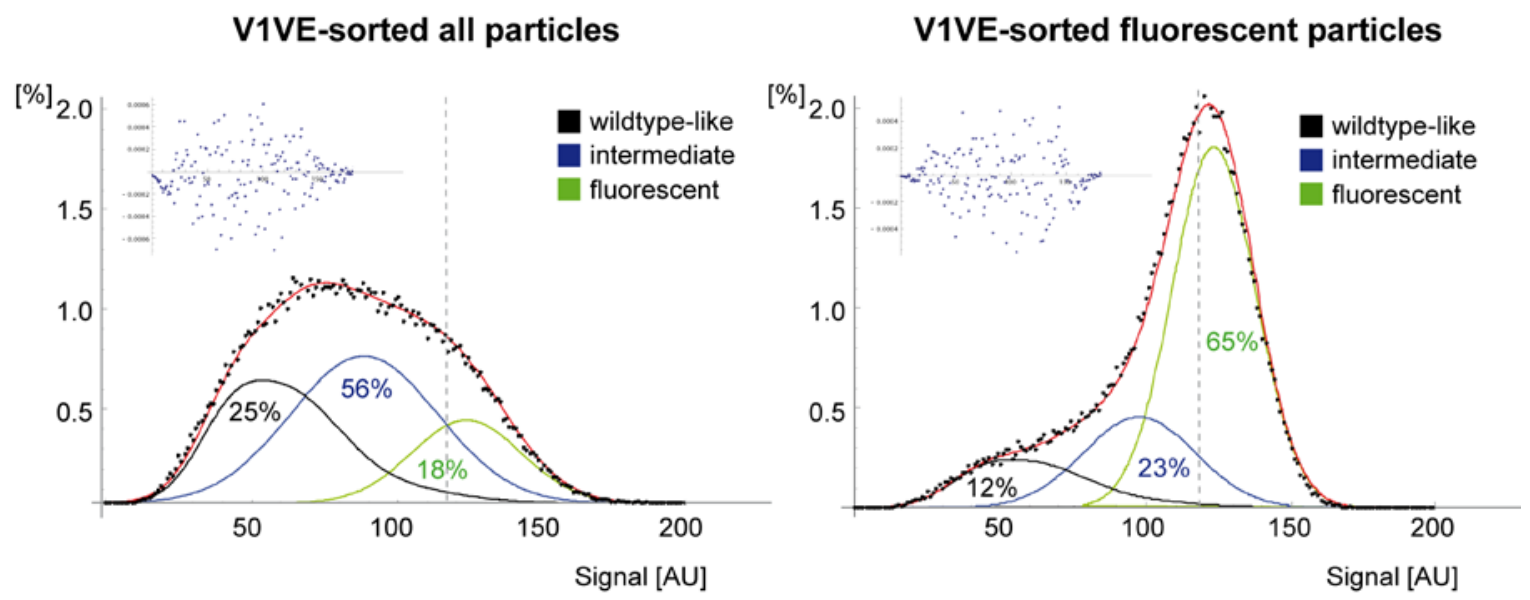

C

enrichment-depletion

sorted fluorescent vs. sorted all particles

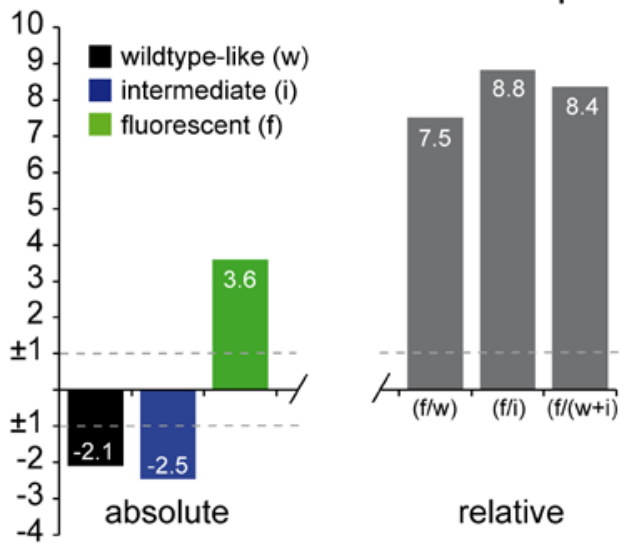




\section{Figure 17: Modeling the flow-data as a mixture of normal distributions}

The fluorescence intensity distribution of sorted VGLUT1 $1^{\text {VENUS }}$ all particles and sorted VGLUT1 $1^{\text {VENUS }}$ fluorescent particles was modeled as a multiple normal distribution components after the data transformed in a 'logicle' display way according to Parks et al. (2006) (see 2.4.3) (A) Data from four independent wild type measurements were fitted separately. In each case a minimum of three normal distribution components was necessary to produce best fit. The averaged wild type probability distribution function (PDF) represents a wild-type-like component in the fitting of VGLUT1 ${ }^{\text {VENUS }}$ synaptosome data (see B). A representative wild-type fit is shown in this panel. The fluorescence threshold used in the analysis of flow cytometry data in sorting of VGLUT1 ${ }^{\text {VENUS }}$ synaptosomes is indicated. (B) The wild-type PDF and additional two normal distributions were necessary to produce an optimal fit for the data from sorted VGLUT1 ${ }^{\text {VENUS }}$ all - particles and sorted VGLUT1 ${ }^{\text {VENUS }}$ fluorescent particles samples. The proportion of the wild-type PDF was applied as a free parameter in the fitting. The three populations were designated as wild-type-like, intermediate and fluorescent according to their mean fluorescence signals. Four independent data sets were fitted and the average proportion of each population is indicated in the graphs. The graphs display a representative fit of the data and the fit residual in the scatter plots at the top of each graph (The fit-residual calculates the difference between the actual data and the fitted data to indicate the goodness of the fit: A random distribution of the difference around the $x$-axis suggests an optimal fit reached.). (C) The absolute change in the proportion of each of the three populations was calculated as the ratio of sortedfluorescent over sorted-all particles. For ratios below one, the reciprocal value was plotted to the negative direction to indicate depletion. Also the change in the relative proportion of the fluorescent component to the other components was determined and the change between the sorted fluorescent particles and the sorted all particles was calculated. 


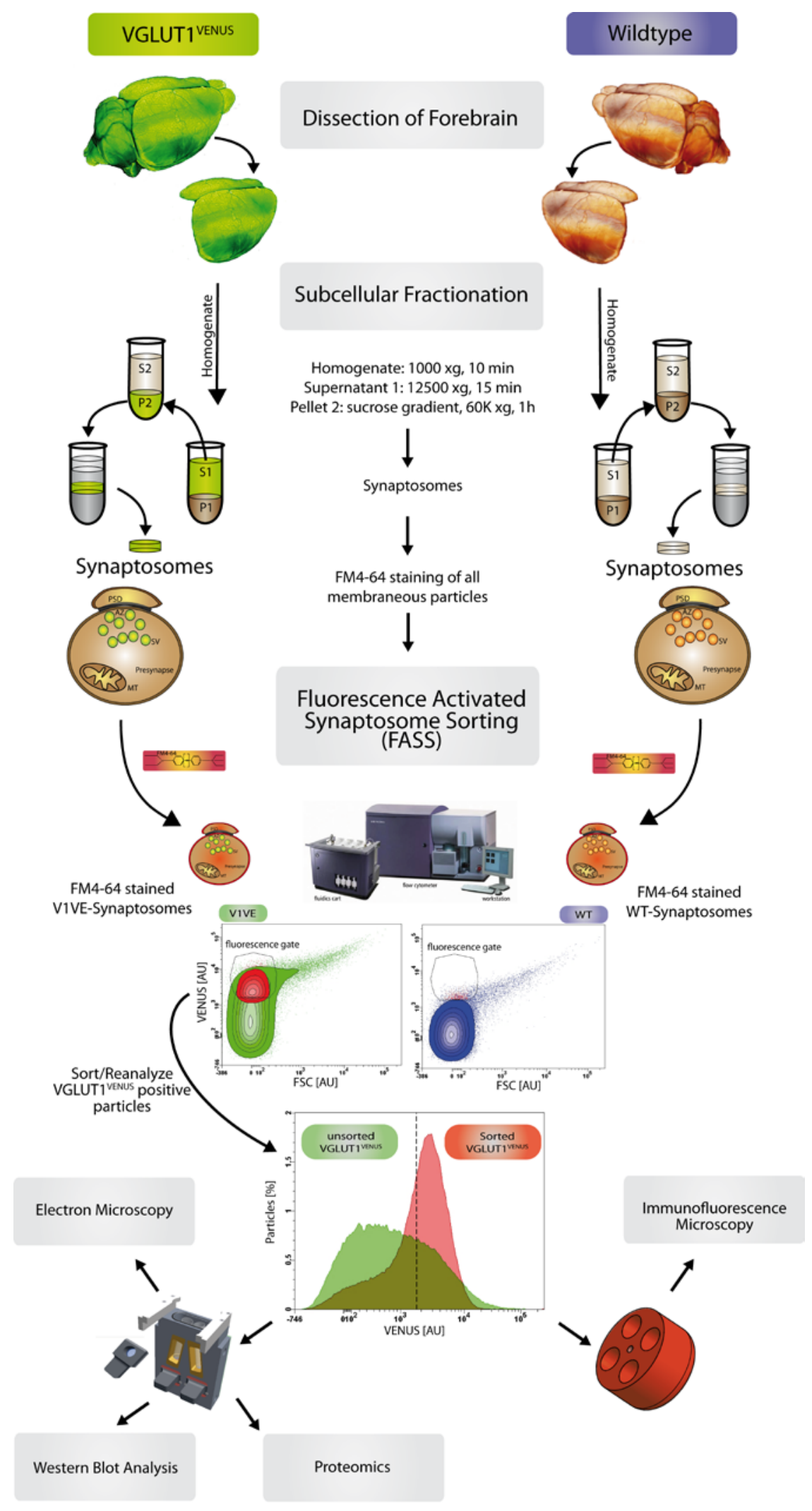




\section{Figure 18: Flow chart of final FASS procedure}

This flow chart demonstrates the procedure of FASS of VGLUT1 ${ }^{\text {VENUS }}$ synaptosomes in its final version. Following FASS the procedure is validated by electron microscopy, Western blot analysis, proteomic analysis and immunofluorescence microscopy using the sample recovery strategies introduced earlier.

\subsubsection{FASS sample protein concentration can be titrated by silver staining and analyzed quantitatively by Western blotting}

In FSC-mode many contaminating sub-threshold particles were co-sorted, which was not the case in the FM-mode. In addition, the sorted particles in FM-mode are of a smaller size. Therefore the protein yield per sort experiment is significantly lower in FM-mode. FASS samples could only be recovered from the polycarbonate filters by use of detergents and not with salt buffers (data not shown). In addition, colorimetric assays that were compatible with the use of detergents did not yield reproducible results or required the use of a large amount of FASS sample (data not shown). Because of the low total protein yield after FASS and the requirement to conserve sample for the later analyses, an estimation of protein concentration in sorted material was done by titration against known concentrations of unsorted synaptosomes in silver stained 1-D-SDS poly-acrylamide gels (Figure 19A). In this way I estimated that the protein yield lies between 0.01 and $0.02 \mu \mathrm{g}$ of protein per million synaptosomes. Due to the low protein yield I always pooled samples from two sort experiments. One third of the sample was titrated on a silver gel, while the other two thirds were used for analysis. The silver gel titration can only provide a rough estimate of protein concentration. Therefore, I chose to quantify the enrichment and depletion of the different proteins relative to the enrichment of VGLUT1 ${ }^{\text {VENUS, }}$, by comparing the ratio of immunoreactivity of a given marker protein to VGLUT1 $1^{\text {VENUS }}$ before and after sorting. By this quantification a protein that co-enriches more strongly than VGLUT1 ${ }^{\text {VENUS }}$ will have a value above 1 , while a protein that co-enriches to a similar extent as VGLUT1 ${ }^{\text {VENUS }}$ will have a value around 1, and a protein that is depleted will have a value below 1. 

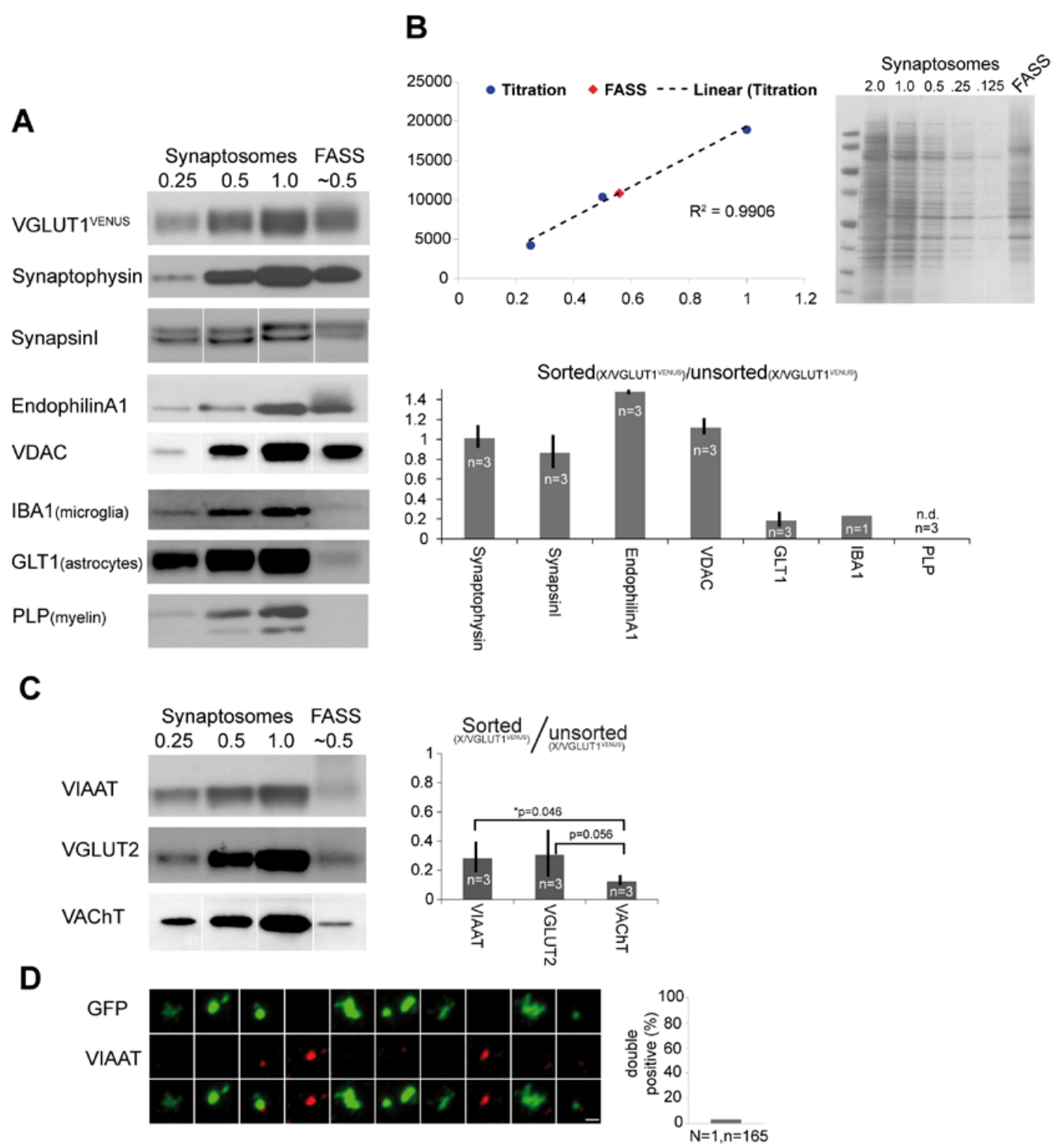

\section{Figure 19: Analysis of synaptic and non-neuronal proteins after FASS}

Following FASS, samples were analyzed by Western blotting and immunofluorescence staining to characterize the composition of the isolated particles. (A) Western blot analysis of Synaptophysin, Synapsinl, EndophilinA1 and VDAC, which are expected to be present at VGLUT1 synapses. Also IBA1, GLT1 and PLP were analyzed, which are proteins specific to microglia, astrocytes and myelin, respectively. The Western blots show $0.25,0.5$ and $1 \mu \mathrm{g}$ of unsorted gradient purified synaptosomes, followed by a FASS sample, which was adjusted to contain $0.5 \mu \mathrm{g}$ of protein according to silver gel titration (see B). The ratio of a given protein to VGLUT1 $1^{\text {VENUS }}$ in the FASS samples was quantified in relation to the ratio of this protein to VGLUT1 ${ }^{\text {VENUS }}$ in the unsorted sample, thereby indicating the co-enrichment or depletion of these proteins (see graph to the right of the blots). (B) Sorted samples were titrated against known amounts of unsorted synaptosomal protein to ensure comparable sample loading in Western blot experiments. (C) Western blot analysis of the neurotransmitter transporters VIAAT, VGLUT2 and VAChT in unsorted synaptosomes and in the FASS sample (see also quantification to the right). Significance of the stronger depletion of VAChT compared to VIAAT and VGLUT2 was tested using a one sided t-test. (D) Immunofluorescence analysis of VGLUT1 ${ }^{\text {VENUS }}$ positive particles after FASS. For each particle the colocalization with VIAAT was analyzed and quantified. $2 \%$ of the particles were positive for both VGLUT1 ${ }^{\text {VENUS }}$ and VIAAT. Scale bar, $1 \mu \mathrm{m}$ 


\subsubsection{FASS co-enriches markers of VGLUT1 synapses and depletes components of microglia, astrocytes and myelin}

The Western blot analysis of FSC-mode fluorescent activated sorting of VGLUT1 ${ }^{\text {VENUS }}$ synaptosomes showed an enrichment of VGLUT1 $1^{\text {VENUS }}$ but also of VIAAT, and a strong enrichment of PLP (Figure 14). In contrast, after FASS in FM-mode PLP was no longer detectable and VIAAT was strongly depleted (Figure $19 \mathrm{~B}, \mathrm{C}$ ). On average, the ratio of VGLUT1 ${ }^{\text {VENUS }}$ to VIAAT was increased 3-fold in the sorted synaptosomes compared to the Bfraction (see graph in Figure $19 \mathrm{C}$ ). These results indicate that FASS in FM-mode successfully enriches single VGLUT1 ${ }^{\text {VENUS }}$ synaptosomes while depleting other types of synaptosomes as well as contaminations from myelin.

If FASS indeed enriches intact VGLUT1 $1^{\text {VENUS }}$ synaptosomes, other proteins present in these synapses should also be co-enriched. Synaptophysin is a very specific marker for synaptic vesicles and coexists with VGLUT1 in excitatory synaptic vesicles (for review see (Valtorta et al., 2004)). Synapsinl is a synaptic vesicle associated phoshpoprotein which is specifically localized to synapses ((De Camilli et al., 1983) for review see (Evergren et al., 2007)). A recent and very thoroughly executed colocalization study demonstrated that more than $91.3 \%$ of VGLUT1 synapses in the cortex contain Synapsinl (Bragina et al., 2007). The same study found that $95.5 \%$ of VGLUT1 synapses also contain Synaptophysinl. I found that both proteins are co-enriched with VGLUT1 in FASS samples (Figure 19 B). While Synaptophysin co-enriches with a factor of 1.01 Synapsinl is co-enriched with a factor of 0.86. The soluble protein EndophilinA1 is known to interact with VGLUT1 at excitatory synapses (De Gois et al., 2006; Vinatier et al., 2006; Voglmaier et al., 2006). EndophilinA1 is enriched in FASS sample by a factor of 1.4 and therefore more strongly enriched than VGLUT1 ${ }^{\text {VENUS }}$. Many presynaptic terminals also contain mitochondria, therefore mitochondrial proteins should be co-enriched with VGLUT1 ${ }^{\text {VENUS }}$ by FASS (Cai and Sheng, 2009; Shepherd and Harris, 1998). The voltage gated anion channel (VDAC) is a protein of the outer mitochondrial membrane and was used to analyze the enrichment of mitochondria by FASS of VGLUT1 ${ }^{\text {VENUS }}$ synaptosomes (for reviews see (De Pinto et al., 2010; ShoshanBarmatz et al., 2010)). VDAC was co-enriched with VGLUT1 $1^{\text {VENUS }}$ at a factor of 1.11 . In addition, TOM20, another mitochondrial protein was analyzed in one experiment and found to be co-enriched with a factor of 0.76 (data not shown).

Apart from synaptic particles, the B-fraction also contains particles from non-neuronal cells, such as astrocytes, microglia and oligodendrocytes (myelin). In the brain, the ionized calcium binding adapter molecule 1 (IBA1) is expressed exclusively in microglial cells (Imai et al., 1996). IBA1 was depleted from the sorted synaptosomes with a ratio of 0.23 (Figure 19 B). GLT1 (EAAT2) is a glial high affinity plasma membrane glutamate transporter in forebrain astrocytes and important for re-uptake of synaptically released glutamate (Lehre 
and Danbolt, 1998; Pines et al., 1992; Rothstein et al., 1996). It can be considered as a specific marker of astrocytic plasma membranes and is depleted with a ratio of 0.18 by FASS (Figure 19 B). Myelin proteolipid protein (PLP) constitutes more than $50 \%$ of the total myelin protein in the CNS and was therefore used as a marker for myelin contaminations (for review (Greer and Lees, 2002)). PLP, which is already heavily depleted during the sucrose-density gradient centrifugation (data not shown), was further depleted by FASS and not detectable in the sorted sample (Figure 19 B).

\subsubsection{VAChT is significantly more depleted than VIAAT or VGLUT2}

Several functionally distinct synapse types exist in the forebrain, which can be classified according to the neurotransmitter they release. The type of vesicular neurotransmitter transporter expressed is a major determinant of a synaptic neurotransmitter phenotype (for review see (Masson et al., 1999)). The excitatory synapses containing VGLUT1 are the most abundant synapses in the mouse forebrain (Bellocchio et al., 1998; Fremeau et al., 2004b). However, there are also excitatory synapses in the forebrain which express VGLUT2 as the major VGLUT isoform (Fremeau et al., 2004b; Herzog et al., 2001). At inhibitory synapses the neurotransmitters Glycine and GABA are transported into synaptic vesicles by VIAAT (Sagné et al., 1997; Wojcik et al., 2006). The cholinergic synapses, in turn, can be labeled using antibodies against the vesicular acetylcholine transporter (VAChT). Therefore, vesicular neurotransmitter transporters are useful markers to study enrichment and depletion of synaptosomes with different neurotransmitter phenotype during FASS. FASS of VGLUT1 ${ }^{\text {VENUS }}$ synaptosomes leads to a 3-fold depletion of VIAAT (0.28) and VGLUT2 (0.30) relative to VGLUT1 ${ }^{\text {VENUS }}$. The 8 -fold depletion of VAChT in the sorted material is significantly stronger than the depletion of VIAAT. Using a quantitative immunofluorescence assay I confirmed that the majority of VGLUT1 ${ }^{\text {VENUS }}$ positive particles in the sorted fraction were negative for VIAAT. When VIAAT positive puncta were found they were usually negative for VGLUT1 ${ }^{\text {VENUS }}$ (Figure $19 \mathrm{D}$ ).

\subsubsection{FASS reports differential expression patterns of SNAP25/SNAP23, $S V 2 A / B$ and $C P X I / I I$ at excitatory terminals}

In the brain several families of synaptic proteins show differential synaptic expression patterns. For SNAP25/SNAP23, SV2A/B and for CPXI/II, differences in localization to excitatory and inhibitory synapses have been suggested on the basis of microscopic studies and synaptic vesicle immunoisolations (Bajjalieh et al., 1994; Bragina et al., 2007; Eastwood and Harrison, 2005; Grønborg et al., 2010; Takahashi et al., 1995). In order to further validate the quality of my purification protocol, we tested, whether FASS can contribute to the characterization of the cellular and subcellular distribution of these proteins (Figure 20). 
SNAP25 showed a trend to be more strongly enriched in sorted VGLUT1 VENUS synaptosomes than SNAP23, indicating that SNAP25 is more strongly associated with VGLUT1 synapses than SNAP23 (Figure 20). However this difference was not statistically significant and also SNAP23 is coenriched with VGLUT1 in comparison to VIAAT or nonsynaptic proteins (Figure 19). Our results therefore confirm earlier results that reported $94 \%$ of VGLUT1 synapses were positive for SNAP25, while only $18 \%$ of VAAT synapses, and 12 $\%$ of VGLUT2 synapses were positive for SNAP25 (Bragina et al., 2007). In the same study it was reported that only $60 \%$ of VGLUT1 synapses co-express SNAP23, while $83 \%$ of VGLUT2 synapses co-express SNAP23. The fact that SNAP23 is also strongly co-enriched with VGLUT1 is not consistent with the reported $60 \%$ of colocalization in the study of Bragina et al. (2007). It is of note that the Western blot signal for SNAP25 is much stronger than the one observed for SNAP23. If this is also the case for immunofluorescence stainings, lower levels of SNAP23 present at VGLUT1 synapses might have escaped proper immunodetection in former anatomical studies (Bragina et al., 2007). In addition, I also investigated the distribution of SNAP47, which has been described as a more or less ubiquitous SNARE with only limited synaptic localization (Holt et al., 2006). SNAP47 is enriched to a similar extent as VGLUT1, indicating that SNAP47 is present in VGLUT1 synapses.

Contrary to SV2B, SV2A did not co-enrich with VGLUT1 ${ }^{\text {VENUS }}$ in the sorted synaptosomes. The difference in enrichment of SV2A and SV2B was statistically significant and indicates that SV2B is likely to be a selective component of VGLUT1 synapses, while SV2A is either ubiquitously localized to all synapses or only present in a subset of VGLUT1 synapses.

Complexinll was co-enriched to a similar extent as VGLUT1 ${ }^{\text {VENUS }}$, while Complexinl was depleted 2-fold and therefore statistically significantly more depleted than Complexinll. Thus, like to the SV2B, Complexinll seems to be more specific for VGLUT1 synapses than Complexinl. 


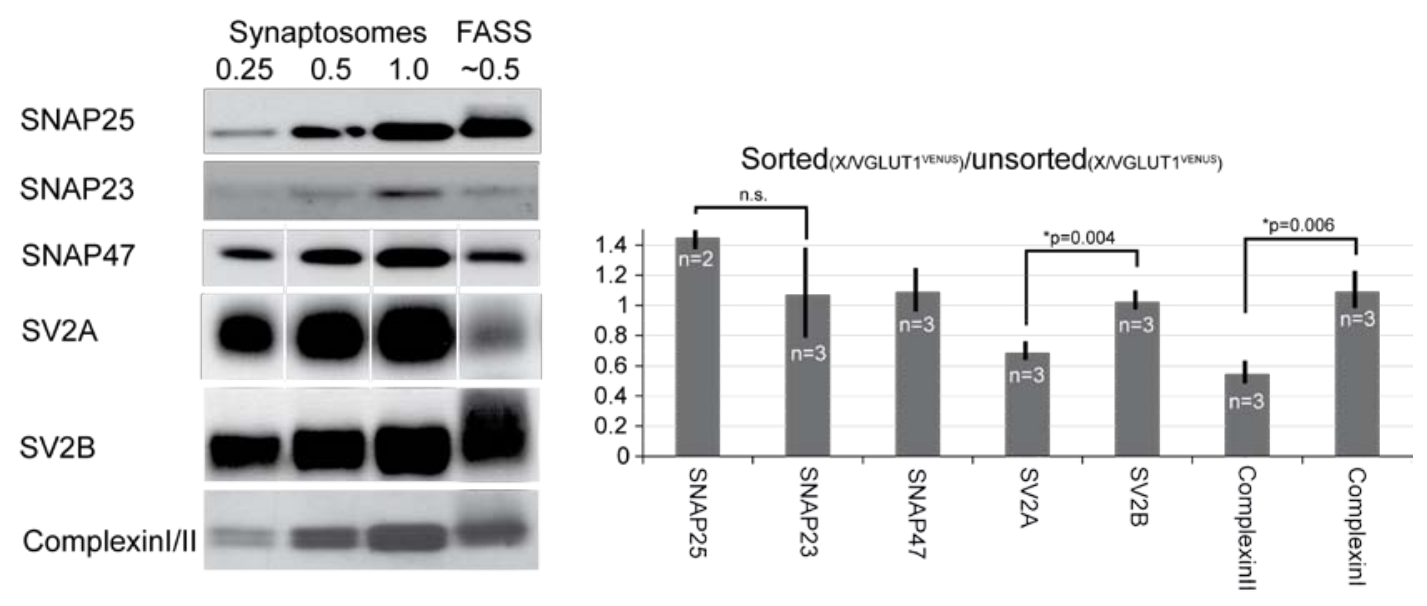

Figure 20: Western blot analysis of FASS elucidates the differential distribution of protein isoforms

Western blot analysis to test the differential distribution of SNAP25 and SNAP23, SNAP47, SV2A and SV2B, and Complexinl and Complexinll in FASS samples compared to unsorted synaptosomes (see Figure 19). For each pair of protein isoforms the stronger depletion of one isoform was tested using a one-sided t-test (n.s.-not significant).

\subsubsection{At least $75 \%$ of FASS-purified VGLUT1 ${ }^{\text {VENUS }}$ synaptosomes have a PSD}

It has been qualitatively established based on EM observations, that synaptosomes generally also have postsynaptic elements attached. However, the fraction of synaptosomes that have a PSD has not been analyzed so far. I therefore performed double immunofluorescence stainings on sorted VGLUT1 ${ }^{\text {VENUS }}$ particles by staining for VGLUT1 ${ }^{\text {VENUS }}$ (GFP antibody) and PSD95 (Figure 21 A). A quantification of three independent experiments, counting a total of 485 particles, showed that around $75 \%$ of VGLUT1 ${ }^{\text {VENUS }}$ positive particles were also positive for PSD95. This implies that at least $75 \%$ of sorted VGLUT1 ${ }^{\text {VENUS }}$ synaptosomes have a PSD attached. FASS therefore also allows the analysis of the biochemical composition of the PSDs attached to VGLUT1 ${ }^{\text {VENUS }}$ synaptosomes. 
A

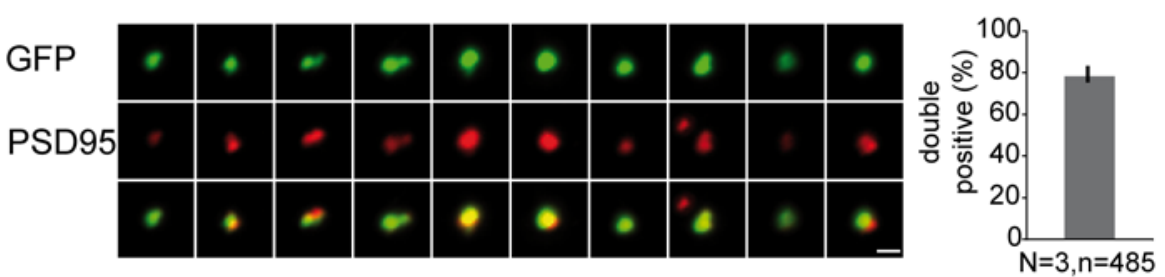

B

\section{Synaptosomes FASS $\begin{array}{llll}0.25 & 0.5 & 1.0 & \sim 0.5\end{array}$}

Neuroligin 1

Neuroligin 2

Neuroligin 3

Neuroligin 4

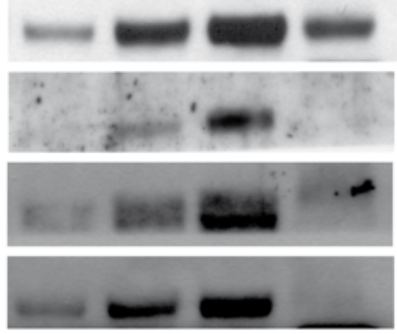

C

GluRII

NMDAR1

NMDAR2a

NMDAR2b
Synaptosomes FASS
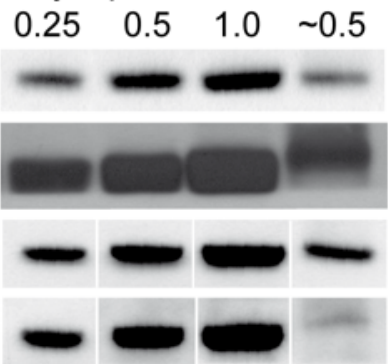

Sorted(XNGLUT1 1vens)/unsorted(XNGLUT1 1 venvs)

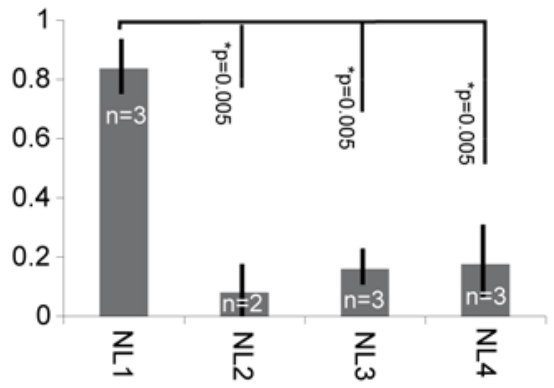

Sorted(XNGLUT1VENus)/unsorted(XNGLUT1VENus)

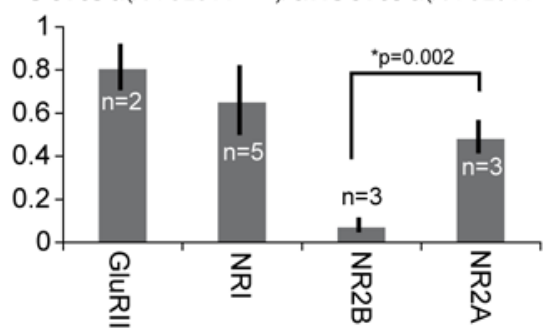

Figure 21: Analysis of PSD95, Neuroligins and glutamate receptors after FASS

The association of postsynaptic proteins with the sorted VGLUT1 ${ }^{\text {VENUS }}$ synaptosomes was analyzed by immunofluorescence staining and Western blotting after FASS. (A) Immunofluorescence staining of VGLUT1 ${ }^{\text {VENUS }}$ synaptosomes after FASS using antibodies against GFP and PSD95. The GFP positive particles were analyzed for their PSD95 fluorescence. The quantification shows the percentage of double positive particles in this analysis. Scale bar, $1 \mu \mathrm{m}$ (B) Analysis of Neuroligin1-4 after FASS. The significance of the depletion of NL2, NL3 and NL4 compared to NL1 was tested using a one-sided t-test. (C) Analysis of AMPA and NMDA receptor subunits after FASS. The significance of the difference in enrichment of NR2A and NR2B was tested using a one-sided t-test.

\subsubsection{Analysis of Neuroligin1-4 after FASS reveals that Neuroligin3 and 4 are mainly localized to non- VGLUT1 synapses}

The analysis of sorted VGLUT1 ${ }^{\text {VENUS }}$ synaptosomes by double immunofluorescence staining showed that an analysis of the postsynaptic density of VGLUT1 ${ }^{\text {VENUS }}$ synaptosomes should be possible after FASS. One prominent family of PSD proteins are the Neuroligins (for review (Lisé and El-Husseini, 2006; Südhof, 2008) ). In the mouse there are four Neuroligin isoforms, Neuroligin1-4. Neuroligins play a role in synapse formation and, more 
importantly, in synapse maturation and function (Varoqueaux et al., 2006). Neuroligin1 is known to be specific for excitatory synapses, while Neuroligin2 is known to be specific for inhibitory synapses (Song et al., 1999; Varoqueaux et al., 2004). The exact cellular and subcellular distributions of Neuroligin3 and Neuroligin4 proteins in the CNS have not been studied to this date. However, both proteins can be detected in Western blots of brain tissue and studies of mRNA expression indicate that they are expressed in neurons throughout the brain. Importantly, mutations in the Neuroligin 3 and 4 genes have been linked to familial cases of autism spectrum disorders (Jamain et al., 2003). Therefore, I chose to investigate the presence of different Neuroligins in VGLUT1 ${ }^{\text {VENUS }}$ synaptosomes.

As expected Neuroligin1 was co-enriched with VGLUT1 ${ }^{\text {VENUS }}$, while Neuroligin2 was strongly depleted. The enrichment ratio for Neuroligin1 was 0.85 and that for Neuroligin2 was less than 0.1, Neuroligin2 was statistically significantly more depleted. Both Neuroligin3 and Neuroligin4 were statistically significantly more depleted from sorted VGLUT1 VENUS synaptosomes than Neuroligin1, but with enrichment ratios of 0.18 and 0.2 they were apparently less drastically excluded from VGLUT1 ${ }^{\text {VENUS }}$ synaptosomes than Neuroligin2.

\subsubsection{FASS selectively enriches the synaptic pool of glutamate receptors}

At excitatory synapses the glutamate released from the presynaptic terminal binds to postsynaptic glutamate receptors (for review see (Traynelis et al., 2010)). Ionotropic AMPA receptors mediate fast excitatory neurotransmission, while NMDA receptors are also important for long-term changes in synaptic transmission. Depending on their subunit composition and subcellular localization glutamate receptors fulfill different functions in synaptic transmission. For example evidence from pharmacology and microscopy indicates that in adult synapses NR2A containing NMDA receptors are concentrated in the postsynaptic density (PSD), while NR2B containing receptors function extrasynaptically (for review see (van Zundert et al., 2004)). However, in quantitative biochemical studies on PSD proteins from synaptic plasma membranes, obtained based on their insolubility in TritonX100 , both NR2A and NR2B containing NMDA receptors were found to be enriched in PSDs to a similar degree and present in similar amounts (reviewed in (Okabe, 2007)). My analysis of VGLUT1 $1^{\text {VENUS }}$ after FASS revealed that at least $75 \%$ of sorted VGLUT1 ${ }^{\text {VENUS }}$ synaptosomes carry immunoreactivity for the PSD protein PSD-95 (Figure $21 \mathrm{~A}$ ). Therefore I used FASS to analyze the subcellular distribution of different AMPA- and NMDA-receptor subunits in sorted VGLUT1 ${ }^{\text {VENUS }}$ synaptosomes and re-addressed the question of differential subsynaptic localization of NR2A and NR2B containing NMDA-receptors (Figure 21 B).

GluRII is coenriched with VGLUT1 ${ }^{\text {VENUS }}$ in sorted VGLUT1 VENUS synaptosomes with a coenrichment factor of 0.8, while NR1, the constitutive subunit of NMDA-receptors, is coenriched with a factor of 0.65 . The NR2A subunit has a coenrichment factor of 0.5 and is 
therefore significantly more enriched than the NR2B subunit, which has a coenrichment factor of 0.1. This indicates that most of the NR2B subunits are located away from VGLUT1 synapses and VGLUT1 post-synaptic densities, either at VGLUT2 synapses or in the extra synaptic compartment.

\subsubsection{Electron microscopy shows integrity and higher purity of synaptosomes after FASS}

As outlined in the previous paragraphs, FASS of VGLUT1 $1^{\text {VENUS }}$ synaptosomes coenriches proteins that mark different parts of the synapse. The FASS enrichment of proteins, that label synaptic vesicles, synaptic cytosol, mitochondria and postsynaptic densities indicates that the isolated particles are predominantly intact synaptosomes. At the same time, many membrane-containing contaminations are depleted. I therefore tested whether the integrity and purity of the sorted synaptosomes can also be demonstrated in electron microscopy. To this end, I concentrated the FASS samples by vacuum filtration on polycarbonate filters. The protocol was the same as the one used for concentrating the FASS sample for Western blotting (Figure 13). The filters were then processed for electron microscopy. Intact synaptosomes were observed in the EM after FASS (Figure 22). In addition to structures that can be identified as synaptosomes, I also observed membranecontaining structures that did not appear to be synaptosomes. Since it was difficult to further classify these particles, they were collectively classified as debris (Figure 22). In a blind observer study, I systematically imaged several regions of the filter surface after sorting "all particles" or only the fluorescent VGLUT1 ${ }^{\text {VENUS }}$ synaptosomes. In each image synaptic or non-synaptic structures were delineated and the respective structures were counted and their area in the image was quantified. By this analysis it was twice as likely to find a synaptic particle in the sorted fluorescent VGLUT1 $1^{\text {VENUS }}$ synaptosomes, than in the "unsorted" sample, where all particles were non-selectively sorted (graph in Figure 22). Strikingly, the ratio of area of synaptic structures versus area of debris particles was increased by a factor of 4.87 . This means that each synaptic particle should yield much more biological material than a contaminating particle in the purified sample. 


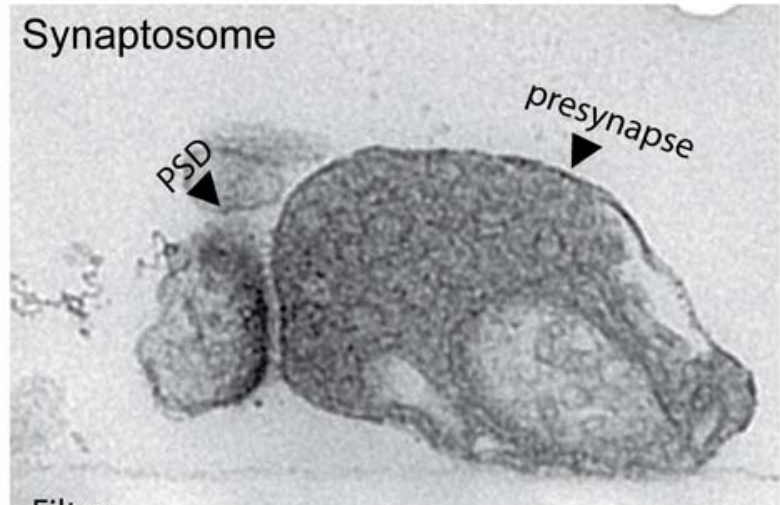

Filter

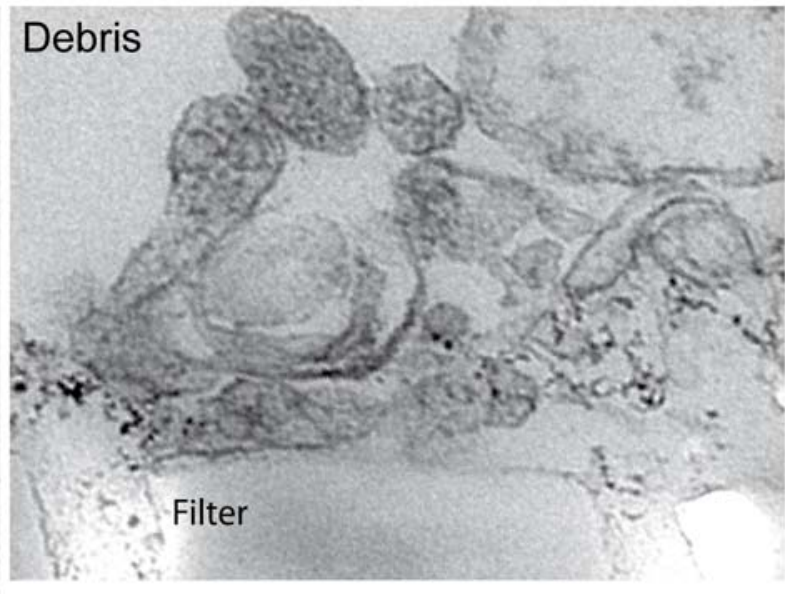

Quantification

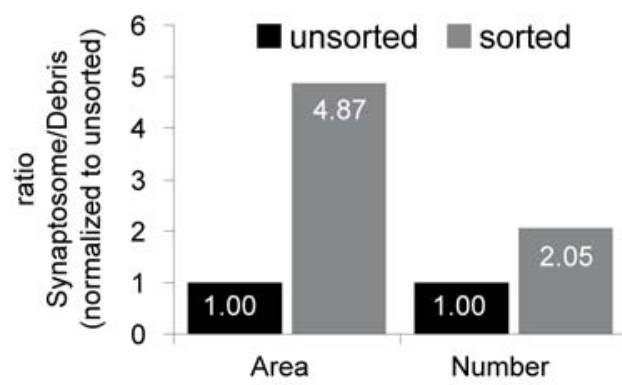

\section{Figure 22: Electron microscopy after FASS}

Electron micrographs of particles concentrated onto Isopore ${ }^{\mathrm{TM}}$ filters after FASS. For the 'unsorted' sample all particles detected in the FACSAria were used. For the 'sorted' sample, the VGLUT1 $1^{\text {VENUS }}$ fluorescent particles were selectively sorted (see Figure 16). Both samples were imaged and quantified blindly, to avoid bias. Both samples contained particles of around $500 \mathrm{~nm}$, surrounded by a plasma membrane and containing a number of vesicles, and occasionally presenting a PSD (see top left, bottom left). In addition to synaptic profiles, non-synaptic 'debris' particles were observed (see top right). The position of the filter is indicated. The quantification determined the area and number of synaptic and debris particles. Values were normalized to the unsorted sample

\subsection{A proteomic screening of sorted VGLUT1 ${ }^{\text {VENUS }}$ synaptosomes}

One motivation to develop protocols for the isolation of specific subpopulations of synaptosomes from mouse brain was to enable an analysis of such pure synaptosomes and their biochemical composition in greater detail without contribution from contaminating structures. Such data should allow the identification of new structural or functional synapse components. The identification of such proteins will motivate further studies on the complexity of synapse function. Furthermore, a comparison of sorted synaptosomes from genetically modified animals (e.g. disease models, knock-out mice) with sorted wild type synaptosomes should allow studying the effects of a certain condition/mutation on the biochemical composition of specific subsets of synapses. The most promising technique for 
accomplishing this analysis is the identification and quantification of proteins by mass spectrometry. The field of mass-spectrometry based protein identification and quantification, often referred to as proteomics, is advancing rapidly. New instrumentation and software applications allow the rapid analysis of more and more complex samples from less and less starting material.

Therefore I also chose to analyze and compare sorted VGLUT1 ${ }^{\text {VENUS }}$ synaptosomes with unsorted gradient purified synaptosomes using proteomic techniques in order to address the following questions:

o Can proteomic techniques be used to analyze FASS samples?

o Is protein enrichment/depletion as assessed by proteomics consistent with the Western blot analysis?

o Which proteins are enriched/depleted after FASS?

o Can FASS help to identify new components of VGLUT1 synapse?

Highly sensitive proteomic approaches like the one used here often generate a large number of putative target proteins that might be of interest for further analysis. However, the interpretation of these candidate collections is often difficult. I therefore employed a variety of different analyses in order to aid the interpretation of the proteome data in the context of the attempted enrichment of VGLUT1-synapse specific proteins. These analyses included semiquantitative analysis by spectral counting and a comparison with available transcriptome data. 


\section{Proteomics screen}

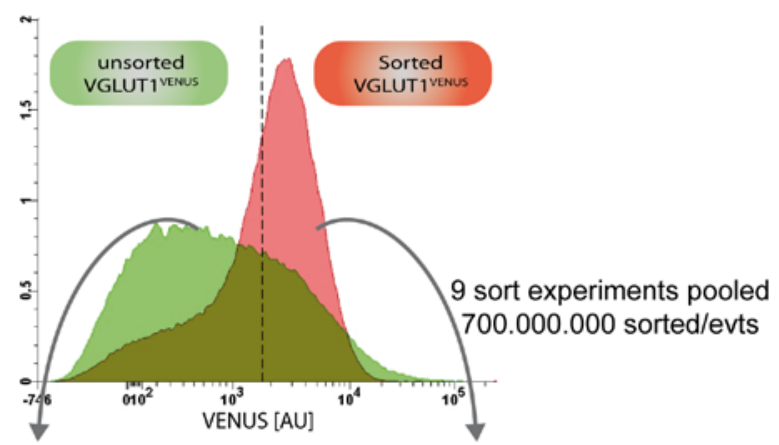

$8 \mu \mathrm{g}$

$8 \mu \mathrm{g}$

unsorted VGLUT1 1 VENUS sorted VGLUT1 1 VENUS

synaptosomes

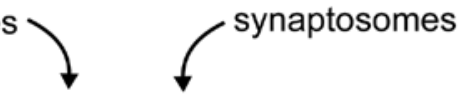

4-12 \% BIS-TRIS SDS-PAGE (Novex ${ }^{\text {TM }}$ )

Each lane cut into 24 pieces

in-gel tryptic digest

peptide extraction (ACN/FA)
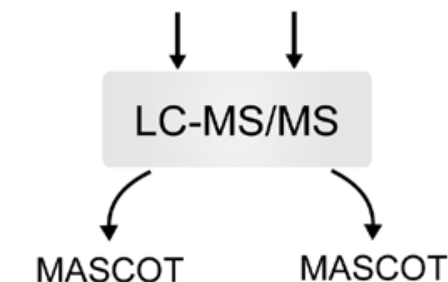

2333 Proteins 2044 Proteins

relative quantification of 1075 proteins by

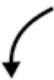

Spectral Counting

343 proteins

depleted from sorted

(2x)

163 proteins enriched in sorted

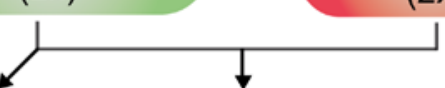

Literature research manual annotation

Confirmation

by

Comparison

to

Western blotting

transcriptome data

Cahoy et. al.

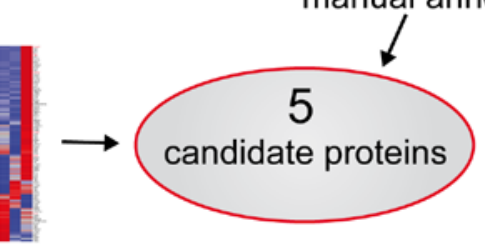

\section{Figure 23 Proteomics screen overview}

Flow chart outlining the major steps for the proteomic comparison of unsorted and sorted VGLUT1 ${ }^{\text {VENUS }}$ synaptosomes, including the different strategies for data analysis and candidate screening (see text for details). 


\subsubsection{Proteomics identifies 2044 proteins in sorted VGLUT1 ${ }^{\text {VENUS }}$ and 2333 in unsorted synaptosomes}

In order to analyze the protein complement of sorted VGLUT1 ${ }^{\text {VENUS }}$ synaptosomes I undertook a large scale FASS experiment in which nine independent experiments were pooled to yield a total of 700.000 .000 sorted fluorescent VGLUT1 ${ }^{\text {VENUS }}$ particles (Figure 23). As with previous Western blot experiments, protein amounts in the FASS sample were titrated by 1-D-SDS PAGE using different amounts of unsorted gradient purified synaptosomes (see Figure 19 for similar example and 2.6.2). Using colloidal Coomassie staining, the total amount of protein was estimated to be $8 \mu \mathrm{g}$. Therefore, $8 \mu \mathrm{g}$ of unsorted synaptosomes were processed in parallel to the sorted sample. Using Mascot, 2333 proteins were identified by at least one unique peptide in the unsorted, gradient purified synaptosomes. In the sorted sample, 2044 proteins were identified by at least one unique peptide. The complete lists of identified proteins from both samples is contained in the attached CD and is available for download at: http://www1.em.mpg.de/biesemann2010

\subsubsection{Enrichment and depletion factors of proteins as determined by spectral counting is consistent with results from Western blotting}

The datasets of unsorted and sorted samples were analyzed with analysis software Scaffold. The Scaffold software (Proteome Software, Portland, Oregon, USA) allows integrating and analyzing proteomics data of different experiments, and was used to validate protein identifications derived from MS/MS sequencing results. Scaffold verifies peptide identifications assigned by Mascot using the X!Tandem database searching program. Afterwards, Scaffold probabilistically validates these peptide identifications using PeptideProphet and derives corresponding protein probabilities using ProteinProphet (Nesvizhskii et al., 2003; Searle et al., 2008).

In addition, Scaffold implements semi-quantitative spectral counting to allow quantitative comparisons between samples. Among the different parameters of MS/MS data, such as the number of identified peptides, the sequence coverage and the number of spectra associated with a protein identification (spectral count), only the spectral count has a high correlation with relative protein abundance (Liu et al., 2004). During an LC-MS/MS experiment, the instrument software autonomously selects a subset of precursor ions (peptides) for sequencing by collision induced decay (CID) analysis. Normally, the instrument selects the five most intense (abundant) precursor ions (peptides) for CID analysis. The quantitative comparison of relative protein abundance between samples by spectral counting is based on the hypothesis that the MS/MS sampling rate, i.e. the rate at which a given peptide is selected for CID analysis, is directly related to the abundance of its precursor ion (peptide) (for review see (Lundgren et al., 2010; Zhu et al., 2010). To allow for a better 
comparison between samples the spectral counts of each experiment are normalized to the total number of spectra.

A major source of error in spectral counting is the erroneous association of peptides with a given protein identification (Mueller et al., 2008). Thus, I used Scaffold to limit the quantitative analysis to high quality peptides with a minimum identification probability of 95 $\%$. Moreover, only proteins with at least two high quality peptides and with a $95 \%$ protein identification probability in at least one of the samples were analyzed. After applying these criteria, a total of 1075 proteins were analyzed for their enrichment or depletion in the sorted VGLUT1 ${ }^{\text {VENUS }}$ synaptosomes by spectral counting using the Scaffold software. 163 proteins were enriched by a factor of two or more in the sorted sample, while 343 proteins were depleted by a factor of two or more. The enriched proteins are displayed in Table2. The complete results of the quantification by spectral counting are contained within the attached CD and available for download at: http://www1.em.mpg.de/biesemann2010

Several proteins, that had previously been analyzed for their enrichment and depletion in Western blotting or by immunofluorescence staining, were above the threshold of 2-fold enrichment or depletion in spectral counting. VGLUT1 was enriched by a factor of 2. Synaptophysin, EndophilinA1, SNAP25, SV2A, SV2B and PSD-95 are all enriched by factor of 2 or more. Therefore the spectral-counting data of these proteins is in agreement with their coenrichement in sorted VGLUT1 ${ }^{\text {VENUS }}$ synaptosomes as assessed by western blotting and immunofluorescence microscopy (Figure 19 -12). Neuroligin2 was depleted with a factor of 3.3, while Neuroligin3 was depleted by a factor of 2.5. PLP was depleted by a factor of 10 . Thus also the spectral counting data that indicates the depletion of proteins in VGLUT1 1 VENUS synaptosomes isolated by FASS is consistent with the results obtained by Western blotting ((Figure 19 and Figure 21) A number of proteins that I have previously analyzed by Western blotting were below the threshold of 2-fold change in the spectral counting data. Synapsin 1 was enriched by a factor of 1.66, SNAP47 by 1.1, Complexinll by 1.43 , VDAC by 1.66 and NR1 by 1.25. On the other hand Complexinl, NR2B and GluRIl were depleted by a factor of 1.1 and GLT-1 was depleted 1.2-fold. Even though the enrichment of these proteins in the VGLUT1 ${ }^{\text {VENUS }}$ synaptosomes as analyzed by spectral counting was below 2, the trends of either enrichment or depletion of proteins is consistent with the Western blot data of FASS samples in all cases except for GluRII (Figure 19 -21). 


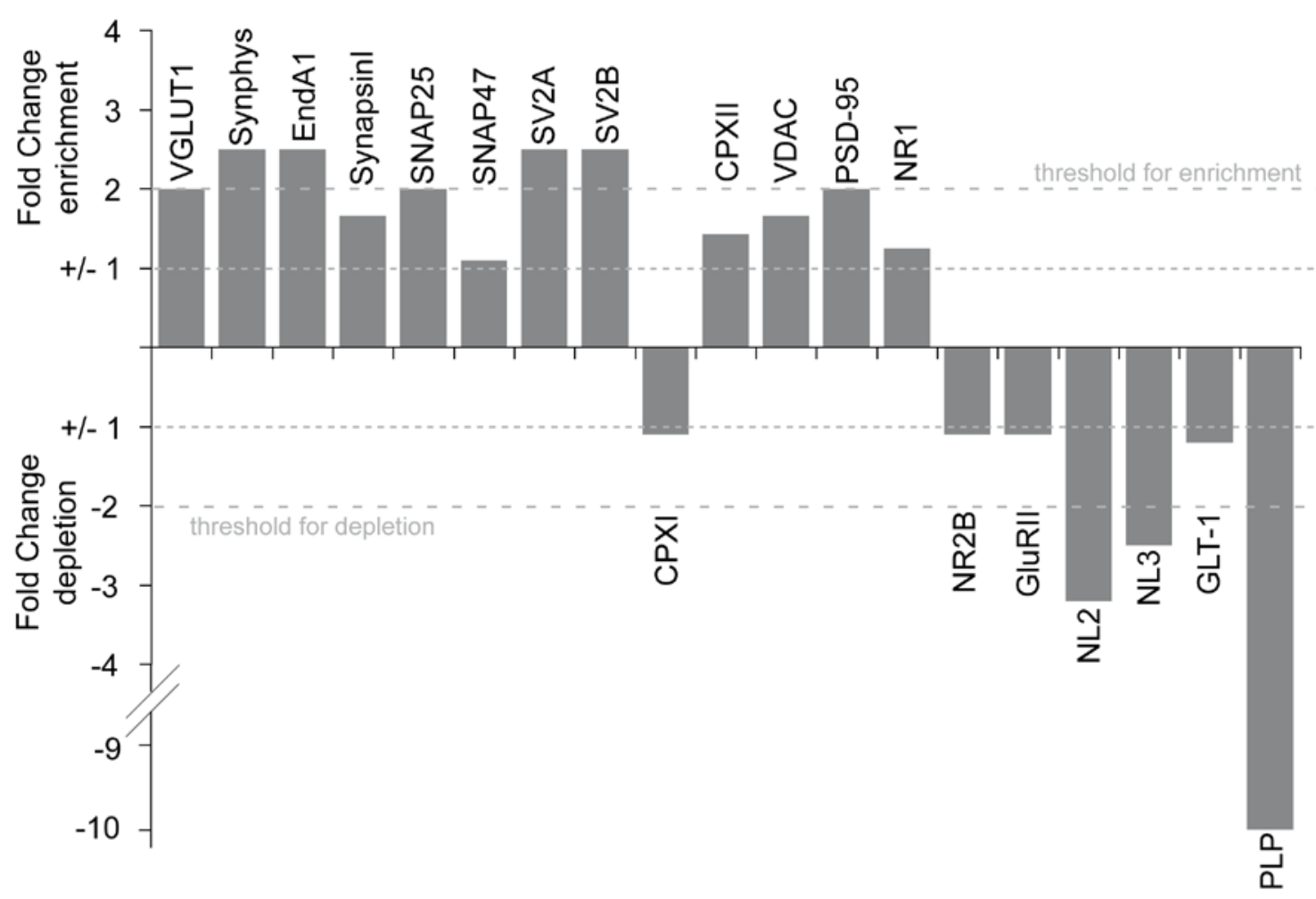

Figure 24: Fold change in protein levels between sorted and unsorted samples as assessed by spectral counting

The fold-change of all proteins analyzed by Western blotting and quantified by spectral counting is displayed. Proteins with a fold change of higher than 1 were plotted with positive values (enriched side). For fold change values below one, the reciprocal values were plotted as negative values (depleted side). Therefore, values of 1 and -1 would be equal (first dashed line). After the analysis by spectral counting a threshold of two-fold change was chosen in order to define a group of protein that are enriched and a group of proteins that are depleted according to the spectral counting data. The threshold is indicated in the figure. A two-fold change was suggested as threshold by the analysis software Scaffold and also corresponds to the enrichment score of VGLUT1 in the sorted VGLUT1 ${ }^{\text {VENUS }}$ synaptosomes as assessed by spectral counting. 


\section{Table 2: Proteins enriched after FASS of VGLUT1 ${ }^{\text {VENUS }}$ synaptosomes}

This Table lists the proteins that were determined to be enriched in the sorted sample according to spectral counting. The gene symbols, gi-numbers and protein names are listed together with the fold change (FC) in normalized spectral counts between sorted VGLUT1 ${ }^{\text {VENUS }}$ synaptosomes (FASS) and unsorted gradient purified synaptosomes (Unsorted) is listed in column four. The following two columns contain the normalized spectral counts (SC), followed by the number of peptides used for quantification (\#pept) and the protein identification probability in percent (pID) as determined by the Scaffold $\otimes$ software for the unsorted and the FASS sample respectively.

\begin{tabular}{|c|c|c|c|c|c|}
\hline $\begin{array}{l}\text { Gene } \\
\text { Symbol }\end{array}$ & $\begin{array}{c}\text { gi } \\
\text { number }\end{array}$ & Protein Name & FC & $\begin{array}{l}\text { Unsorted } \\
\text { SC (\#pept) } \\
\text { pID\% }\end{array}$ & $\begin{array}{c}\text { FASS } \\
\text { SC (\#pept) } \\
\text { pID\% }\end{array}$ \\
\hline Actb & 6671509 & actin, beta & 2.00 & $\begin{array}{c}295.93(24) \\
100 \%\end{array}$ & $\begin{array}{l}591.23(28) \\
100 \%\end{array}$ \\
\hline Camk2a & 28916677 & $\begin{array}{l}\text { calcium/calmodulin-dependent protein kinase II alpha } \\
\text { isoform } 2\end{array}$ & 2.00 & $\begin{array}{l}233.26(30) \\
100 \%\end{array}$ & $\begin{array}{l}507.77(29) \\
100 \%\end{array}$ \\
\hline Stxbp1 & 165972305 & syntaxin binding protein 1 isoform b (Munc18-1) & 2.00 & $\begin{array}{c}242.55(49) \\
100 \%\end{array}$ & $\begin{array}{l}492.84(51) \\
100 \%\end{array}$ \\
\hline Syt1 & 6678197 & synaptotagmin I & 2.50 & $\begin{array}{c}143.90(23) \\
100 \%\end{array}$ & $\begin{array}{l}381.27(29) \\
100 \%\end{array}$ \\
\hline Actc1 & 14192922 & actin, alpha, cardiac muscle 1 & 2.50 & $\begin{array}{c}160.15(3) \\
100 \%\end{array}$ & $\begin{array}{l}362.82(2) \\
100 \%\end{array}$ \\
\hline Atp6v1a & 31560731 & ATPase, $\mathrm{H}+$ transporting, lysosomal V1 subunit $\mathrm{A}$ & 2.00 & $\begin{array}{c}153.19(38) \\
100 \%\end{array}$ & $\begin{array}{l}322.41(39) \\
100 \%\end{array}$ \\
\hline Nsf & 31543349 & vesicle-fusing ATPase (NSF) & 2.00 & $\begin{array}{l}143.90(52) \\
100 \%\end{array}$ & $\begin{array}{l}270.58(52) \\
100 \%\end{array}$ \\
\hline Atp6v1b2 & 19705578 & vacuolar H+ATPase B2 & 2.50 & $\begin{array}{c}98.64(27) \\
100 \%\end{array}$ & $\begin{array}{l}253.01(26) \\
100 \%\end{array}$ \\
\hline Camk2b & 226693349 & calcium/calmodulin-dependent protein kinase II beta & 2.00 & $\begin{array}{c}123.01(13) \\
100 \%\end{array}$ & $\begin{array}{l}238.07(14) \\
100 \%\end{array}$ \\
\hline Actbl2 & 30425250 & actin, beta-like 2 & 2.00 & $\begin{array}{l}99.80(2) \\
100 \%\end{array}$ & $\begin{array}{l}216.99(1) \\
100 \%\end{array}$ \\
\hline Stx1b & 13259378 & syntaxin 1B & 2.00 & $\begin{array}{l}90.52(11) \\
100 \%\end{array}$ & $\begin{array}{l}198.54(13) \\
100 \%\end{array}$ \\
\hline Camk2d & 70906479 & $\begin{array}{l}\text { calcium/calmodulin-dependent protein kinase II, delta } \\
\text { isoform } 1\end{array}$ & 2.00 & $\begin{array}{c}102.12(3) \\
100 \%\end{array}$ & $\begin{array}{l}197.66(4) \\
100 \%\end{array}$ \\
\hline Camk2g & 75991700 & $\begin{array}{l}\text { calcium/calmodulin-dependent protein kinase II gamma } \\
\text { isoform } 1\end{array}$ & 2.00 & $\begin{array}{l}77.75(1) \\
99 \%\end{array}$ & $\begin{array}{l}164.28(2) \\
100 \%\end{array}$ \\
\hline Dlg4 & 6681195 & post-synaptic density protein 95 isoform 1 & 2.00 & $\begin{array}{l}76.59(29) \\
100 \%\end{array}$ & $\begin{array}{c}149.34(30) \\
100 \%\end{array}$ \\
\hline Vamp2 & 6678551 & vesicle-associated membrane protein 2 & 2.50 & $\begin{array}{l}58.03(6) \\
100 \%\end{array}$ & $\begin{array}{c}143.20(6) \\
100 \%\end{array}$ \\
\hline Snap25 & 6755588 & synaptosomal-associated protein 25 & 2.00 & $\begin{array}{c}71.95(16) \\
100 \%\end{array}$ & $\begin{array}{l}135.29(21) \\
100 \%\end{array}$ \\
\hline Syp & 166235165 & synaptophysin & 2.50 & $\begin{array}{l}47.58(6) \\
100 \%\end{array}$ & $\begin{array}{c}132.65(9) \\
100 \%\end{array}$ \\
\hline Arf1 & 194353962 & ADP-ribosylation factor 1 & 2.00 & $\begin{array}{l}60.35(13) \\
100 \%\end{array}$ & $\begin{array}{l}128.26(14) \\
100 \%\end{array}$ \\
\hline Stx1a & 15011853 & syntaxin $1 \mathrm{~A}$ (brain) & 2.50 & $\begin{array}{c}42.94(13) \\
100 \%\end{array}$ & $\begin{array}{l}116.84(23) \\
100 \%\end{array}$ \\
\hline Rab3b & 12963723 & RAB3B, member RAS oncogene family & 2.00 & $\begin{array}{l}60.35(3) \\
100 \%\end{array}$ & $\begin{array}{l}115.08(6) \\
100 \%\end{array}$ \\
\hline Sh3gl2 & 31560792 & SH3-domain GRB2-like 2 (EndophilinA1) & 2.50 & $\begin{array}{l}41.78(13) \\
100 \%\end{array}$ & $\begin{array}{l}109.81(16) \\
100 \%\end{array}$ \\
\hline Hspa2 & 31560686 & heat shock protein 2 & 2.00 & $\begin{array}{l}48.74(1) \\
50 \%\end{array}$ & $\begin{array}{l}103.66(2) \\
100 \%\end{array}$ \\
\hline Sv2a & 11528518 & synaptic vesicle glycoprotein 2 a & 2.50 & $\begin{array}{l}35.98(7) \\
100 \%\end{array}$ & $\begin{array}{l}94.00(13) \\
100 \%\end{array}$ \\
\hline Hsp90aa1 & 6754254 & heat shock protein 1 , alpha & 2.00 & $\begin{array}{c}46.42(13) \\
100 \%\end{array}$ & $\begin{array}{c}90.49(25) \\
100 \%\end{array}$ \\
\hline Slc17a7 & 218156282 & solute carrier family 17 , member 7 (VGLUT1) & 2.00 & $\begin{array}{l}37.14(8) \\
100 \%\end{array}$ & $\begin{array}{l}69.40(11) \\
100 \%\end{array}$ \\
\hline Ppp2r1a & 8394027 & alpha isoform of regulatory subunit $A$, protein phosph & 2.00 & $\begin{array}{c}31.33(14) \\
100 \%\end{array}$ & $\begin{array}{c}65.01(18) \\
100 \%\end{array}$ \\
\hline
\end{tabular}




\begin{tabular}{|c|c|c|c|c|c|}
\hline $\begin{array}{l}\text { Gene } \\
\text { Symbol }\end{array}$ & $\begin{array}{c}\text { gi } \\
\text { number }\end{array}$ & Protein Name & FC & $\begin{array}{l}\text { Unsorted } \\
\text { SC (\#pept) } \\
\text { pID\% }\end{array}$ & $\begin{array}{c}\text { FASS } \\
\text { SC (\#pept) } \\
\text { pID\% }\end{array}$ \\
\hline Arf5 & 6680722 & ADP-ribosylation factor 5 & 3.33 & $\begin{array}{l}19.73(2) \\
100 \%\end{array}$ & $\begin{array}{l}59.74(3) \\
100 \%\end{array}$ \\
\hline Atp6v0c & 6753144 & ATPase, $\mathrm{H}+$ transporting, lysosomal V0 subunit $\mathrm{C}$ & 2.50 & $\begin{array}{l}22.05(3) \\
100 \%\end{array}$ & $\begin{array}{l}52.71(3) \\
100 \%\end{array}$ \\
\hline Hspa1b & 124339826 & heat shock $70 \mathrm{kDa}$ protein $1 \mathrm{~B}$ & 2.00 & $\begin{array}{c}24.37(3) \\
100 \%\end{array}$ & $\begin{array}{l}50.95(3) \\
100 \%\end{array}$ \\
\hline Dlg2 & 118136297 & chapsyn-110 (PSD-93) & 2.50 & $\begin{array}{l}22.05(10) \\
100 \%\end{array}$ & $\begin{array}{c}49.20(17) \\
100 \%\end{array}$ \\
\hline Cadps & 70906474 & $\begin{array}{c}\mathrm{Ca}<2+\text { dependent activator protein for secretion isoform } 1 \\
\text { (CAPS1) }\end{array}$ & 5.00 & $\begin{array}{l}8.12(6) \\
100 \%\end{array}$ & $\begin{array}{l}45.68(16) \\
100 \%\end{array}$ \\
\hline Arf4 & 6680720 & ADP-ribosylation factor 4 & 3.33 & $\begin{array}{l}12.77(1) \\
50 \%\end{array}$ & $\begin{array}{l}42.17(2) \\
100 \%\end{array}$ \\
\hline Sv2b & 157909825 & synaptic vesicle glycoprotein $2 \mathrm{~b}$ & 2.50 & $\begin{array}{l}18.57(5) \\
100 \%\end{array}$ & $\begin{array}{l}42.17(8) \\
100 \%\end{array}$ \\
\hline Oxr1 & 194328708 & oxidation resistance 1 isoform $E$ & 2.50 & $\begin{array}{l}13.93(9) \\
100 \%\end{array}$ & $\begin{array}{l}39.53(18) \\
100 \%\end{array}$ \\
\hline Ctnna2 & 157951727 & catenin, alpha 2 isoform 1 & 2.50 & $\begin{array}{l}13.93(6) \\
100 \%\end{array}$ & $\begin{array}{l}34.26(16) \\
100 \%\end{array}$ \\
\hline Hpcal4 & 27923929 & hippocalcin-like 4 & 2.50 & $\begin{array}{l}12.77(4) \\
100 \%\end{array}$ & $\begin{array}{l}33.38(11) \\
100 \%\end{array}$ \\
\hline Arpc2 & 112363072 & actin related protein $2 / 3$ complex, subunit 2 & 2.00 & $\begin{array}{c}16.25(10) \\
100 \%\end{array}$ & $\begin{array}{c}30.75(10) \\
100 \%\end{array}$ \\
\hline Actr3b & 52345394 & ARP3 actin-related protein 3 homolog B & 5.00 & $\begin{array}{l}4.64(1) \\
50 \%\end{array}$ & $\begin{array}{c}27.23(6) \\
100 \%\end{array}$ \\
\hline Vat1l & 33667095 & vesicle amine transport protein 1 homolog-like (T. californica) & 5.00 & $\begin{array}{c}4.64(3) \\
100 \%\end{array}$ & $\begin{array}{c}25.48(6) \\
100 \%\end{array}$ \\
\hline Syngr3 & 31560541 & synaptogyrin 3 & 2.50 & $\begin{array}{c}9.28(4) \\
100 \%\end{array}$ & $\begin{array}{c}25.48(6) \\
100 \%\end{array}$ \\
\hline Atp6v1f & 21314824 & ATPase, $\mathrm{H}+$ transporting, lysosomal V1 subunit $\mathrm{F}$ & 2.50 & $\begin{array}{c}9.28(2) \\
100 \%\end{array}$ & $\begin{array}{c}25.48(9) \\
100 \%\end{array}$ \\
\hline Ap2m1 & 6753074 & adaptor protein complex AP-2, mu1 & 2.50 & $\begin{array}{c}9.28(4) \\
100 \%\end{array}$ & $\begin{array}{c}24.60(12) \\
100 \%\end{array}$ \\
\hline Plcb1 & 224967070 & phospholipase $\mathrm{C}$, beta 1 isoform 1 & 10.00 & $\begin{array}{c}1.16(1) \\
100 \%\end{array}$ & $\begin{array}{c}23.72(14) \\
100 \%\end{array}$ \\
\hline Syngr1 & 46877048 & synaptogyrin 1 isoform $1 \mathrm{a}$ & 2.50 & $\begin{array}{c}9.28(2) \\
100 \%\end{array}$ & $\begin{array}{c}23.72(2) \\
100 \%\end{array}$ \\
\hline Sh3gl1 & 7305485 & SH3-domain GRB2-like 1 (Endophilin2) & 2.00 & $\begin{array}{c}11.61(0) \\
0 \%\end{array}$ & $\begin{array}{c}23.72(6) \\
100 \%\end{array}$ \\
\hline Vamp1 & 6678549 & vesicle-associated membrane protein 1 isoform a & 2.50 & $\begin{array}{c}9.28(2) \\
100 \%\end{array}$ & $\begin{array}{c}22.84(3) \\
100 \%\end{array}$ \\
\hline Erc2 & 37360977 & ELKS/RAB6-interacting/CAST family member 2 (CASK) & 3.33 & $\begin{array}{c}5.80(4) \\
100 \%\end{array}$ & $\begin{array}{c}21.96(17) \\
100 \%\end{array}$ \\
\hline Lyz1 & 7305247 & lysozyme 1 & 3.33 & $\begin{array}{c}5.80(2) \\
100 \%\end{array}$ & $\begin{array}{c}21.08(4) \\
100 \%\end{array}$ \\
\hline Ap2s1 & 161086984 & adaptor-related protein complex 2 , sigma 1 subunit & 3.33 & $\begin{array}{c}6.96(4) \\
100 \%\end{array}$ & $\begin{array}{c}21.08(6) \\
100 \%\end{array}$ \\
\hline Anks1b & 189303581 & ankyrin repeat and sterile alpha motif domain containing $1 \mathrm{~B}$ & 2.00 & $\begin{array}{c}10.44(5) \\
100 \%\end{array}$ & $\begin{array}{c}21.08(7) \\
100 \%\end{array}$ \\
\hline Ly6h & 149266963 & Lymphocyte antigen $6 \mathrm{H}(\mathrm{Ly}-6 \mathrm{H})$ & 5.00 & $\begin{array}{c}3.48(2) \\
100 \%\end{array}$ & $\begin{array}{c}20.21(5) \\
100 \%\end{array}$ \\
\hline Dmxl2 & 189491684 & Dmx-like 2 (rabconectin3) & 5.00 & $\begin{array}{c}3.48(3) \\
100 \%\end{array}$ & $\begin{array}{c}19.33(11) \\
100 \%\end{array}$ \\
\hline Hebp1 & 124001580 & heme binding protein 1 & 2.50 & $\begin{array}{c}8.12(5) \\
100 \%\end{array}$ & $\begin{array}{c}18.45(10) \\
100 \%\end{array}$ \\
\hline Rap1gds1 & 100818161 & RAP1, GTP-GDP dissociation stimulator 1 isoform a & 2.50 & $\begin{array}{c}6.96(5) \\
100 \%\end{array}$ & $\begin{array}{c}18.45(14) \\
100 \%\end{array}$ \\
\hline Ppfia3 & 161016786 & $\begin{array}{l}\text { protein tyrosine phosphatase, receptor type, f polypeptide } \\
\text { (PTPRF), interacting protein (liprin), alpha } 3\end{array}$ & 2.00 & $\begin{array}{c}9.28(7) \\
100 \%\end{array}$ & $\begin{array}{c}18.45(14) \\
100 \%\end{array}$ \\
\hline Tom112 & 84875524 & target of myb1-like 2 isoform b & 3.33 & $\begin{array}{l}5.80(2) \\
100 \%\end{array}$ & $\begin{array}{l}16.69(2) \\
100 \%\end{array}$ \\
\hline Lancl2 & 19526936 & LanC (bacterial lantibiotic synthetase component C)-like 2 & 3.33 & $\begin{array}{c}4.64(2) \\
100 \%\end{array}$ & $\begin{array}{c}16.69(9) \\
100 \%\end{array}$ \\
\hline Ppp2r2a & 110625886 & $\begin{array}{l}\text { alpha isoform of regulatory subunit B55, } \\
\text { protein phosphatase } 2\end{array}$ & 2.00 & $\begin{array}{l}8.12(4) \\
100 \%\end{array}$ & $\begin{array}{l}16.69(4) \\
100 \%\end{array}$ \\
\hline Atp5i & 83715998 & $\begin{array}{c}\text { ATP synthase, } \mathrm{H}+\text { transporting, mitochondrial F1F0 complex, } \\
\text { subunit e }\end{array}$ & 2.00 & $\begin{array}{l}8.12(3) \\
100 \%\end{array}$ & $\begin{array}{l}16.69(7) \\
100 \%\end{array}$ \\
\hline Ndufb7 & 13385322 & NADH dehydrogenase (ubiquinone) 1 beta subcomplex, 7 & 5.00 & $\begin{array}{l}3.48(2) \\
100 \%\end{array}$ & $\begin{array}{l}15.81(7) \\
100 \%\end{array}$ \\
\hline Slc8a1 & 119120890 & $\begin{array}{l}\text { solute carrier family } 8 \text { (sodium/calcium exchanger), member } 1 \\
\text { isoform A }\end{array}$ & 2.00 & $\begin{array}{l}8.12(3) \\
100 \%\end{array}$ & $\begin{array}{l}15.81(3) \\
100 \%\end{array}$ \\
\hline
\end{tabular}




\begin{tabular}{|c|c|c|c|c|c|}
\hline $\begin{array}{l}\text { Gene } \\
\text { Symbol }\end{array}$ & $\begin{array}{c}\text { gi } \\
\text { number }\end{array}$ & Protein Name & FC & $\begin{array}{l}\text { Unsorted } \\
\text { SC (\#pept) } \\
\text { pID\% }\end{array}$ & $\begin{array}{c}\text { FASS } \\
\text { SC (\#pept) } \\
\text { pID\% }\end{array}$ \\
\hline Hprt & 96975138 & hypoxanthine phosphoribosyltransferase 1 & 2.00 & $\begin{array}{l}8.12(5) \\
100 \%\end{array}$ & $\begin{array}{l}15.81(9) \\
100 \%\end{array}$ \\
\hline Myh10 & 33598964 & myosin heavy chain 10, non-muscle & 5.00 & $\begin{array}{l}3.48(2) \\
100 \%\end{array}$ & $\begin{array}{c}14.93(11) \\
100 \%\end{array}$ \\
\hline Syt12 & 19527400 & synaptotagmin XII & 5.00 & $\begin{array}{l}2.32(1) \\
50 \%\end{array}$ & $\begin{array}{l}14.06(6) \\
100 \%\end{array}$ \\
\hline Ppm1h & 160358866 & protein phosphatase $1 \mathrm{H}$ (PP2C domain containing) isoform 1 & 2.50 & $\begin{array}{c}4.64(2) \\
100 \%\end{array}$ & $\begin{array}{l}13.18(10) \\
100 \%\end{array}$ \\
\hline Mtnd4 & 34538607 & NADH dehydrogenase subunit 4 & 2.00 & $\begin{array}{l}6.96(1) \\
50 \%\end{array}$ & $\begin{array}{c}13.18(3) \\
100 \%\end{array}$ \\
\hline Ndufa9 & 254692859 & NADH dehydrogenase (ubiquinone) 1 alpha subcomplex, 9 & 2.00 & $\begin{array}{l}6.96(5) \\
100 \%\end{array}$ & $\begin{array}{l}13.18(5) \\
100 \%\end{array}$ \\
\hline Gm6265 & 149263103 & PREDICTED: hypothetical protein & 10.00 & $\begin{array}{l}0.00(0) \\
6 \%\end{array}$ & $\begin{array}{l}12.30(6) \\
100 \%\end{array}$ \\
\hline Hmgb1 & 149266674 & high mobility group box 1 & 10.00 & $\begin{array}{l}0.00(0) \\
0 \%\end{array}$ & $\begin{array}{l}11.42(5) \\
100 \%\end{array}$ \\
\hline Nptx1 & 6679120 & neuronal pentraxin 1 & 2.50 & $\begin{array}{c}4.64(3) \\
100 \%\end{array}$ & $\begin{array}{l}11.42(6) \\
100 \%\end{array}$ \\
\hline Ehd3 & 215983062 & EH-domain containing 3 & 2.50 & $\begin{array}{l}4.64(2) \\
100 \%\end{array}$ & $\begin{array}{l}11.42(9) \\
100 \%\end{array}$ \\
\hline Mapre2 & 253314540 & $\begin{array}{l}\text { microtubule-associated protein, RP/EB family, member } 2 \\
\text { isoform } 1\end{array}$ & 2.00 & $\begin{array}{l}5.80(2) \\
100 \%\end{array}$ & $\begin{array}{l}11.42(3) \\
100 \%\end{array}$ \\
\hline Psd3 & 163644296 & pleckstrin and Sec 7 domain containing 3 isoform 3 & 2.00 & $\begin{array}{l}5.80(4) \\
100 \%\end{array}$ & $\begin{array}{l}11.42(7) \\
100 \%\end{array}$ \\
\hline Cox6b1 & 13385090 & cytochrome c oxidase subunit VIb polypeptide 1 & 10.00 & $\begin{array}{c}1.00(0) \\
0 \%\end{array}$ & $\begin{array}{l}10.54(7) \\
100 \%\end{array}$ \\
\hline Pgrmc1 & 31980806 & progesterone receptor membrane component & 5.00 & $\begin{array}{l}2.32(1) \\
99 \%\end{array}$ & $\begin{array}{l}10.54(6) \\
100 \%\end{array}$ \\
\hline Tpd52 & 70608194 & tumor protein D52 isoform 1 & 3.33 & $\begin{array}{l}3.48(2) \\
100 \%\end{array}$ & $\begin{array}{l}10.54(7) \\
100 \%\end{array}$ \\
\hline Ptrf & 6679567 & polymerase I and transcript release factor & 10.00 & $\begin{array}{l}0.00(0) \\
0 \%\end{array}$ & $\begin{array}{l}9.66(3) \\
100 \%\end{array}$ \\
\hline Gng2 & 6754020 & $\begin{array}{c}\text { guanine nucleotide binding protein (G protein), gamma } 2 \\
\text { subunit }\end{array}$ & 10.00 & $\begin{array}{c}1.00(0) \\
0 \%\end{array}$ & $\begin{array}{c}8.78(5) \\
100 \%\end{array}$ \\
\hline Dynll2 & 18087731 & dynein light chain LC8-type 2 & 10.00 & $\begin{array}{l}0.00(0) \\
0 \%\end{array}$ & $\begin{array}{l}8.78(6) \\
100 \%\end{array}$ \\
\hline Scai & 86198339 & suppressor of cancer cell invasion & 3.33 & $\begin{array}{l}2.32(1) \\
50 \%\end{array}$ & $\begin{array}{l}8.78(3) \\
100 \%\end{array}$ \\
\hline Unc13a & 71274170 & unc-13 homolog $A$ & 10.00 & $\begin{array}{l}0.00(0) \\
0 \%\end{array}$ & $\begin{array}{l}7.91(5) \\
100 \%\end{array}$ \\
\hline Ndufa2 & 31981600 & NADH dehydrogenase (ubiquinone) 1 alpha subcomplex, 2 & 10.00 & $\begin{array}{c}1.00(0) \\
0 \%\end{array}$ & $\begin{array}{l}7.91(6) \\
100 \%\end{array}$ \\
\hline Ggt7 & 224994186 & gamma-glutamyltransferase 7 & 3.33 & $\begin{array}{l}2.32(1) \\
50 \%\end{array}$ & $\begin{array}{c}7.91(2) \\
100 \%\end{array}$ \\
\hline Ndufs4 & 6754814 & NADH dehydrogenase (ubiquinone) Fe-S protein 4 & 2.50 & $\begin{array}{c}3.48(2) \\
100 \%\end{array}$ & $\begin{array}{c}7.91(5) \\
100 \%\end{array}$ \\
\hline Cct8 & 126723461 & chaperonin subunit 8 (theta) & 2.50 & $\begin{array}{c}3.48(2) \\
100 \%\end{array}$ & $\begin{array}{c}7.91(7) \\
100 \%\end{array}$ \\
\hline Fam81a & 58037475 & hypothetical protein LOC76886 & 10.00 & $\begin{array}{c}0.00(0) \\
43 \%\end{array}$ & $\begin{array}{c}7.03(4) \\
100 \%\end{array}$ \\
\hline Chmp4b & 28077049 & charged multivesicular body protein $4 \mathrm{~b}$ & 10.00 & $\begin{array}{c}0.00(0) \\
5 \%\end{array}$ & $\begin{array}{c}7.03(5) \\
100 \%\end{array}$ \\
\hline Dynll1 & 213688406 & dynein light chain LC8-type 1 & 5.00 & $\begin{array}{c}1.16(1) \\
50 \%\end{array}$ & $\begin{array}{c}7.03(2) \\
100 \%\end{array}$ \\
\hline Synj2bp & 13384642 & synaptojanin 2 binding protein & 3.33 & $\begin{array}{c}2.32(1) \\
50 \%\end{array}$ & $\begin{array}{c}7.03(4) \\
100 \%\end{array}$ \\
\hline Rasal1 & 31980729 & RAS protein activator like 1 (GAP1 like) & 3.33 & $\begin{array}{c}2.32(2) \\
100 \%\end{array}$ & $\begin{array}{c}7.03(7) \\
100 \%\end{array}$ \\
\hline Ncs1 & 9790115 & frequenin homolog & 2.00 & $\begin{array}{c}3.48(3) \\
100 \%\end{array}$ & $\begin{array}{c}7.03(3) \\
100 \%\end{array}$ \\
\hline Arpc5I & 21312654 & actin related protein $2 / 3$ complex, subunit 5 -like & 2.00 & $\begin{array}{c}3.48(2) \\
100 \%\end{array}$ & $\begin{array}{c}7.03(4) \\
100 \%\end{array}$ \\
\hline $\begin{array}{l}2810405 \\
\text { K02Rik }\end{array}$ & 27754130 & hypothetical protein LOC66469 & 2.00 & $\begin{array}{c}3.48(2) \\
100 \%\end{array}$ & $\begin{array}{c}7.03(5) \\
100 \%\end{array}$ \\
\hline Dctn1 & 118601017 & dynactin 1 & 2.00 & $\begin{array}{c}3.48(2) \\
100 \%\end{array}$ & $\begin{array}{c}7.03(6) \\
100 \%\end{array}$ \\
\hline Arl6ip5 & 14149750 & ADP-ribosylation factor-like 6 interacting protein 5 & 10.00 & $\begin{array}{c}0.00(0) \\
13 \%\end{array}$ & $\begin{array}{c}6.15(2) \\
100 \%\end{array}$ \\
\hline Uqcr10 & 37574048 & ubiquinol-cytochrome $\mathrm{c}$ reductase complex protein & 10.00 & $\begin{array}{c}1.00(0) \\
0 \%\end{array}$ & $\begin{array}{c}6.15(3) \\
100 \%\end{array}$ \\
\hline
\end{tabular}




\begin{tabular}{|c|c|c|c|c|c|}
\hline $\begin{array}{l}\text { Gene } \\
\text { Symbol }\end{array}$ & $\begin{array}{c}\text { gi } \\
\text { number }\end{array}$ & Protein Name & FC & $\begin{array}{l}\text { Unsorted } \\
\text { SC (\#pept) } \\
\text { pID\% }\end{array}$ & $\begin{array}{c}\text { FASS } \\
\text { SC (\#pept) } \\
\text { pID\% }\end{array}$ \\
\hline Gm11275 & 149263932 & histone $\mathrm{H} 4$ & 10.00 & $\begin{array}{c}1.00(0) \\
0 \%\end{array}$ & $\begin{array}{c}6.15(3) \\
100 \%\end{array}$ \\
\hline Gng12 & 13384618 & guanine nucleotide binding protein (G protein), gamma 12 & 10.00 & $\begin{array}{c}1.00(0) \\
0 \%\end{array}$ & $\begin{array}{c}6.15(4) \\
100 \%\end{array}$ \\
\hline Ap3b2 & 52317148 & adaptor-related protein complex 3 , beta 2 subunit & 5.00 & $\begin{array}{c}1.16(1) \\
99 \%\end{array}$ & $\begin{array}{c}6.15(2) \\
100 \%\end{array}$ \\
\hline Rufy3 & 21312036 & RUN and FYVE domain containing 3 & 5.00 & $\begin{array}{c}1.16(1) \\
50 \%\end{array}$ & $\begin{array}{c}6.15(3) \\
100 \%\end{array}$ \\
\hline Nudc & 6754910 & nuclear distribution gene $\mathrm{C}$ homolog & 2.50 & $\begin{array}{c}2.32(1) \\
50 \%\end{array}$ & $\begin{array}{c}6.15(3) \\
100 \%\end{array}$ \\
\hline Csnk2b & 7106277 & casein kinase 2, beta polypeptide & 2.50 & $\begin{array}{c}2.32(1) \\
50 \%\end{array}$ & $\begin{array}{c}6.15(3) \\
100 \%\end{array}$ \\
\hline Smap1 & 28077013 & stromal membrane-associated protein 1 & 2.50 & $\begin{array}{c}2.32(1) \\
100 \%\end{array}$ & $\begin{array}{c}6.15(3) \\
100 \%\end{array}$ \\
\hline Dnaja2 & 9789937 & DnaJ subfamily A member 2 & 2.50 & $\begin{array}{c}2.32(2) \\
100 \%\end{array}$ & $\begin{array}{c}6.15(5) \\
100 \%\end{array}$ \\
\hline $\begin{array}{l}9430016 \\
\text { H08Rik }\end{array}$ & 145587094 & hypothetical protein LOC68115 & 10.00 & $\begin{array}{c}0.00(0) \\
0 \%\end{array}$ & $\begin{array}{c}5.27(2) \\
100 \%\end{array}$ \\
\hline Stx6 & 10946800 & syntaxin 6 & 10.00 & $\begin{array}{c}1.00(0) \\
0 \%\end{array}$ & $\begin{array}{c}5.27(4) \\
100 \%\end{array}$ \\
\hline Grb2 & 6680083 & growth factor receptor bound protein 2 & 5.00 & $\begin{array}{c}1.16(1) \\
50 \%\end{array}$ & $\begin{array}{c}5.27(3) \\
100 \%\end{array}$ \\
\hline Atp6v1d & 12963799 & ATPase, $\mathrm{H}+$ transporting, $\mathrm{V} 1$ subunit $\mathrm{D}$ & 5.00 & $\begin{array}{c}1.16(1) \\
100 \%\end{array}$ & $\begin{array}{c}5.27(4) \\
100 \%\end{array}$ \\
\hline Tpm1 & 256000796 & tropomyosin 1, alpha isoform 10 & 5.00 & $\begin{array}{c}1.16(1) \\
98 \%\end{array}$ & $\begin{array}{c}5.27(5) \\
100 \%\end{array}$ \\
\hline $\begin{array}{l}\text { D230025 } \\
\text { D16Rik }\end{array}$ & 170932498 & $\operatorname{lin}-10$ & 2.50 & $\begin{array}{c}2.32(1) \\
50 \%\end{array}$ & $\begin{array}{c}5.27(2) \\
100 \%\end{array}$ \\
\hline Twf2 & 6755224 & twinfilin-like protein & 2.50 & $\begin{array}{c}2.32(1) \\
50 \%\end{array}$ & $\begin{array}{c}5.27(3) \\
100 \%\end{array}$ \\
\hline Cox7a2 & 31981830 & cytochrome $\mathrm{c}$ oxidase, subunit VIIa 2 & 10.00 & $\begin{array}{c}1.00(0) \\
0 \%\end{array}$ & $\begin{array}{c}4.39(2) \\
100 \%\end{array}$ \\
\hline Atp5j & 7949005 & ATP synthase, $\mathrm{H}+$ transporting, mitochondrial F0 complex, & 10.00 & $\begin{array}{l}1.00(0) \\
0 \%\end{array}$ & $\begin{array}{c}4.39(3) \\
100 \%\end{array}$ \\
\hline Wasf3 & 21553113 & WAS protein family, member 3 & 10.00 & $\begin{array}{c}1.00(0) \\
0 \%\end{array}$ & $\begin{array}{c}4.39(3) \\
100 \%\end{array}$ \\
\hline F8a & 83921576 & factor 8-associated gene A & 10.00 & $\begin{array}{c}1.00(0) \\
0 \%\end{array}$ & $\begin{array}{c}4.39(3) \\
100 \%\end{array}$ \\
\hline Psmd14 & 145966883 & proteasome $26 \mathrm{~S}$ subunit, non-ATPase 14 & 10.00 & $\begin{array}{l}0.00(0) \\
7 \%\end{array}$ & $\begin{array}{c}4.39(4) \\
100 \%\end{array}$ \\
\hline St13 & 19526912 & suppression of tumorigenicity 13 & 3.33 & $\begin{array}{c}1.16(1) \\
50 \%\end{array}$ & $\begin{array}{c}4.39(4) \\
100 \%\end{array}$ \\
\hline Fxyd6 & 11612657 & FXYD domain-containing ion transport regulator 6 & 2.00 & $\begin{array}{l}2.32(1) \\
50 \%\end{array}$ & $\begin{array}{l}4.39(2) \\
100 \%\end{array}$ \\
\hline Nptxr & 36054013 & neuronal pentraxin receptor & 2.00 & $\begin{array}{l}2.32(1) \\
99 \%\end{array}$ & $\begin{array}{l}4.39(3) \\
100 \%\end{array}$ \\
\hline Lypla1 & 6678760 & lysophospholipase 1 & 2.00 & $\begin{array}{c}2.32(2) \\
100 \%\end{array}$ & $\begin{array}{c}4.39(4) \\
100 \%\end{array}$ \\
\hline Cab39 & 161086893 & calcium binding protein 39 & 2.00 & $\begin{array}{l}2.32(2) \\
100 \%\end{array}$ & $\begin{array}{c}4.39(4) \\
100 \%\end{array}$ \\
\hline Gng3 & 6754022 & $\begin{array}{c}\text { guanine nucleotide binding protein (G protein), gamma } 3 \\
\text { subunit }\end{array}$ & 10.00 & $\begin{array}{l}0.00(0) \\
0 \%\end{array}$ & $\begin{array}{c}3.51(2) \\
100 \%\end{array}$ \\
\hline Acyp1 & 13384810 & acylphosphatase 1 & 10.00 & $\begin{array}{l}1.00(0) \\
0 \%\end{array}$ & $\begin{array}{l}3.51(2) \\
100 \%\end{array}$ \\
\hline Erp29 & 19526463 & endoplasmic reticulum protein ERp29 & 10.00 & $\begin{array}{c}1.00(0) \\
0 \%\end{array}$ & $\begin{array}{c}3.51(2) \\
100 \%\end{array}$ \\
\hline Sdpr & 20270267 & serum deprivation response & 10.00 & $\begin{array}{c}0.00(0) \\
0 \%\end{array}$ & $\begin{array}{c}3.51(2) \\
100 \%\end{array}$ \\
\hline Myl12b & 21728376 & myosin, light chain $12 \mathrm{~B}$, regulatory & 10.00 & $\begin{array}{c}1.00(0) \\
0 \%\end{array}$ & $\begin{array}{c}3.51(2) \\
100 \%\end{array}$ \\
\hline $\begin{array}{l}\text { LOC1000 } \\
47590\end{array}$ & 149249181 & PREDICTED: hypothetical protein & 10.00 & $\begin{array}{c}0.00(0) \\
0 \%\end{array}$ & $\begin{array}{c}3.51(2) \\
100 \%\end{array}$ \\
\hline Kalrn & 256017137 & kalirin, RhoGEF kinase & 10.00 & $\begin{array}{c}0.00(0) \\
0 \%\end{array}$ & $\begin{array}{c}3.51(2) \\
100 \%\end{array}$ \\
\hline Uqcrh & 21539599 & ubiquinol-cytochrome $\mathrm{c}$ reductase hinge protein & 10.00 & $\begin{array}{c}0.00(0) \\
99 \%\end{array}$ & $\begin{array}{c}3.51(2) \\
100 \%\end{array}$ \\
\hline Ndrg4 & 21704212 & N-myc downstream regulated gene 4 & 10.00 & $\begin{array}{c}0.00(0) \\
0 \%\end{array}$ & $\begin{array}{c}3.51(3) \\
100 \%\end{array}$ \\
\hline $\begin{array}{l}\text { Tmem } \\
126 a\end{array}$ & 13384870 & transmembrane protein $126 \mathrm{~A}$ & 3.33 & $\begin{array}{c}1.16(1) \\
50 \%\end{array}$ & $\begin{array}{c}3.51(2) \\
100 \%\end{array}$ \\
\hline
\end{tabular}




\begin{tabular}{|c|c|c|c|c|c|}
\hline $\begin{array}{l}\text { Gene } \\
\text { Symbol }\end{array}$ & $\begin{array}{c}\text { gi } \\
\text { number }\end{array}$ & Protein Name & FC & $\begin{array}{l}\text { Unsorted } \\
\text { SC (\#pept) } \\
\text { pID\% }\end{array}$ & $\begin{array}{c}\text { FASS } \\
\text { SC (\#pept) } \\
\text { pID\% }\end{array}$ \\
\hline Sgta & 21313588 & $\begin{array}{l}\text { small glutamine-rich tetratricopeptide repeat (TPR) containing } \\
\text { protein }\end{array}$ & 3.33 & $\begin{array}{c}1.16(1) \\
50 \%\end{array}$ & $\begin{array}{c}3.51(2) \\
100 \%\end{array}$ \\
\hline Psmc6 & 27754103 & proteasome $26 \mathrm{~S}$ ATPase subunit 6 & 3.33 & $\begin{array}{l}1.16(1) \\
50 \%\end{array}$ & $\begin{array}{l}3.51(2) \\
100 \%\end{array}$ \\
\hline Scamp3 & 118601011 & secretory carrier membrane protein 3 & 3.33 & $\begin{array}{l}1.16(1) \\
50 \%\end{array}$ & $\begin{array}{l}3.51(2) \\
100 \%\end{array}$ \\
\hline Cyb5a & 13385268 & cytochrome b-5 & 3.33 & $\begin{array}{c}1.16(1) \\
98 \%\end{array}$ & $\begin{array}{c}3.51(2) \\
100 \%\end{array}$ \\
\hline Sugt1 & 23956176 & SGT1, suppressor of G2 allele of SKP1 & 3.33 & $\begin{array}{c}1.16(1) \\
50 \%\end{array}$ & $\begin{array}{c}3.51(3) \\
100 \%\end{array}$ \\
\hline Kif1a & 160333877 & kinesin family member $1 \mathrm{~A}$ isoform $\mathrm{b}$ & 3.33 & $\begin{array}{c}1.16(1) \\
98 \%\end{array}$ & $\begin{array}{c}3.51(3) \\
100 \%\end{array}$ \\
\hline Lypla2 & 7242156 & Iysophospholipase 2 & 3.33 & $\begin{array}{l}1.16(1) \\
100 \%\end{array}$ & $\begin{array}{c}3.51(3) \\
100 \%\end{array}$ \\
\hline Stxbp5 & 158749547 & syntaxin binding protein 5 (tomosyn) & 10.00 & $\begin{array}{l}0.00(0) \\
0 \%\end{array}$ & $\begin{array}{l}2.64(2) \\
100 \%\end{array}$ \\
\hline Plcxd3 & 169234801 & $\begin{array}{c}\text { phosphatidylinositol-specific phospholipase C, X domain } \\
\text { containing } 3\end{array}$ & 10.00 & $\begin{array}{l}1.00(0) \\
0 \%\end{array}$ & $\begin{array}{l}2.64(2) \\
100 \%\end{array}$ \\
\hline PIs3 & 21704120 & plastin 3 & 10.00 & $\begin{array}{l}0.00(0) \\
0 \%\end{array}$ & $\begin{array}{l}2.64(3) \\
100 \%\end{array}$ \\
\hline Vamp7 & 33468929 & vesicle-associated membrane protein 7 & 10.00 & $\begin{array}{c}0.00(0) \\
0 \%\end{array}$ & $\begin{array}{c}2.64(3) \\
100 \%\end{array}$ \\
\hline Kiaa0513 & 255069800 & hypothetical protein LOC234797 isoform a & 10.00 & $\begin{array}{c}1.00(0) \\
0 \%\end{array}$ & $\begin{array}{c}2.64(3) \\
100 \%\end{array}$ \\
\hline Sgip1 & 21536264 & SH3-domain GRB2-like (endophilin) interacting protein 1 & 2.50 & $\begin{array}{c}1.16(1) \\
50 \%\end{array}$ & $\begin{array}{c}2.64(2) \\
100 \%\end{array}$ \\
\hline Rnf141 & 84781808 & ring finger protein 141 & 2.50 & $\begin{array}{c}1.16(1) \\
50 \%\end{array}$ & $\begin{array}{c}2.64(2) \\
100 \%\end{array}$ \\
\hline Lysmd2 & 130490961 & LysM, putative peptidoglycan-binding, domain containing 2 & 2.50 & $\begin{array}{c}1.16(1) \\
50 \%\end{array}$ & $\begin{array}{c}2.64(2) \\
100 \%\end{array}$ \\
\hline Gm88 & 162287223 & hypothetical protein LOC224813 & 2.50 & $\begin{array}{c}1.16(1) \\
50 \%\end{array}$ & $\begin{array}{c}2.64(2) \\
100 \%\end{array}$ \\
\hline Iqsec1 & 197304784 & IQ motif and Sec7 domain 1 isoform b & 2.50 & $\begin{array}{c}1.16(1) \\
50 \%\end{array}$ & $\begin{array}{c}2.64(2) \\
100 \%\end{array}$ \\
\hline Eif5a & 262359885 & eukaryotic translation initiation factor $5 \mathrm{~A}$ & 2.50 & $\begin{array}{c}1.16(1) \\
50 \%\end{array}$ & $\begin{array}{c}2.64(2) \\
100 \%\end{array}$ \\
\hline Cpe & 22203763 & carboxypeptidase E & 2.50 & $\begin{array}{c}1.16(1) \\
98 \%\end{array}$ & $\begin{array}{c}2.64(3) \\
100 \%\end{array}$ \\
\hline Stmn3 & 6677873 & stathmin-like 3 & 10.00 & $\begin{array}{c}0.00(0) \\
0 \%\end{array}$ & $\begin{array}{c}1.76(2) \\
100 \%\end{array}$ \\
\hline Psmd13 & 6755210 & proteasome $26 \mathrm{~S}$ non-ATPase subunit 13 & 10.00 & $\begin{array}{c}1.00(0) \\
0 \%\end{array}$ & $\begin{array}{c}1.76(2) \\
100 \%\end{array}$ \\
\hline Clvs2 & 30425184 & clavesin 2 & 10.00 & $\begin{array}{c}1.00(0) \\
0 \%\end{array}$ & $\begin{array}{c}1.76(2) \\
100 \%\end{array}$ \\
\hline Cadm1 & 46575940 & cell adhesion molecule 1 isoform $b$ & 10.00 & $\begin{array}{c}0.00(0) \\
0 \%\end{array}$ & $\begin{array}{c}1.76(2) \\
100 \%\end{array}$ \\
\hline Tmem163 & 58037291 & transmembrane protein 163 (SV31) & 10.00 & $\begin{array}{c}1.00(0) \\
0 \%\end{array}$ & $\begin{array}{c}1.76(2) \\
100 \%\end{array}$ \\
\hline Lphn3 & 58037543 & latrophilin 3 & 10.00 & $\begin{array}{c}0.00(0) \\
0 \%\end{array}$ & $\begin{array}{c}1.76(2) \\
100 \%\end{array}$ \\
\hline Camk1d & 79750129 & calcium/calmodulin-dependent protein kinase 1D & 10.00 & $\begin{array}{c}0.00(0) \\
0 \%\end{array}$ & $\begin{array}{c}1.76(2) \\
100 \%\end{array}$ \\
\hline Rimbp2 & 124487059 & RIMS binding protein 2 & 10.00 & $\begin{array}{c}1.00(0) \\
0 \%\end{array}$ & $\begin{array}{c}1.76(2) \\
100 \%\end{array}$ \\
\hline $\begin{array}{l}\text { LOC1000 } \\
43022\end{array}$ & 149251337 & Adhesion regulating molecule 1 isoform 1 & 10.00 & $\begin{array}{c}1.00(0) \\
0 \%\end{array}$ & $\begin{array}{c}1.76(2) \\
100 \%\end{array}$ \\
\hline Gm10086 & 149254156 & PREDICTED: hypothetical protein & 10.00 & $\begin{array}{c}0.00(0) \\
0 \%\end{array}$ & $\begin{array}{c}1.76(2) \\
100 \%\end{array}$ \\
\hline Vps26a & 164518904 & vacuolar protein sorting 26 isoform b & 10.00 & $\begin{array}{c}1.00(0) \\
0 \%\end{array}$ & $\begin{array}{c}1.76(2) \\
100 \%\end{array}$ \\
\hline Rab23 & 228480287 & RAB23, member RAS oncogene family & 10.00 & $\begin{array}{c}0.00(0) \\
0 \%\end{array}$ & $\begin{array}{c}1.76(2) \\
100 \%\end{array}$ \\
\hline Flnc & 124487139 & filamin C, gamma & 10.00 & $\begin{array}{c}0.00(0) \\
15 \%\end{array}$ & $\begin{array}{c}1.76(2) \\
100 \%\end{array}$ \\
\hline
\end{tabular}




\subsubsection{A comparison with transcriptome data of astrocytes, oligodendrocytes and neurons reveals that FASS of VGLUT1 ${ }^{\text {VENUS }}$ synaptosomes are enriched in neuron-specific and depleted in glia-specific proteins.}

As outlined above, conventional synaptosome preparations are typically contaminated by many particles of glial origin. The quantification of the proteome data obtained here showed 163 proteins to be enriched and 343 proteins to be depleted in the VGLUT1 $1^{\text {VENUS }}$ synaptosomes. The depletion of GLT-1, IBA1 and PLP as analyzed by Western blotting, indicates that glial components are depleted in the VGLUT1 ${ }^{\text {VENUS }}$ synaptosome sample. A study reported by Cahoy et al. (2008) analyzed and compared the mRNA expression profiles of astrocytes, oligodendrocytes and neurons isolated from mouse brain (Cahoy et al., 2008). My prediction was that FASS of VGLUT1 ${ }^{\text {VENUS }}$ synaptosomes should lead to enrichment of proteins whose mRNA expression is specific to, or at least high in neurons. At the same time, the mRNAs corresponding to the depleted proteins ought to be predominantly expressed in glia or non-VGLUT1 expressing neurons. To test this hypothesis, I collaborated with Dr. Sven Peter Wichert to compare proteomic data obtained in the present study data to transcriptome data from astrocytes, oligodendrocytes and neurons (Figure 25). 
A

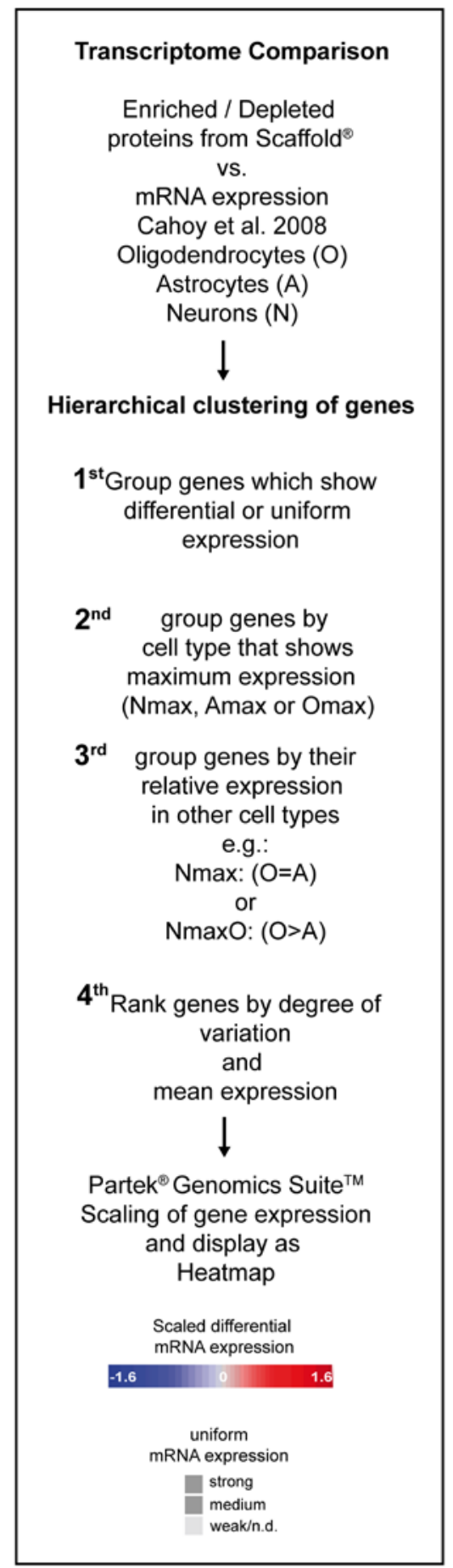

\section{B}

Enriched in proteomics Depleted in proteomics mRNA expression mRNA expression

Oligo Astro Neuro

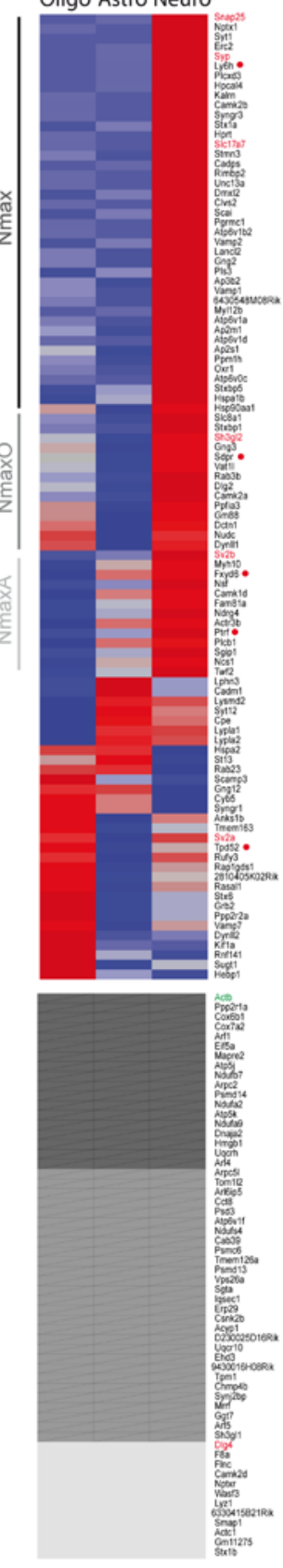

Oligo Astro Neuro

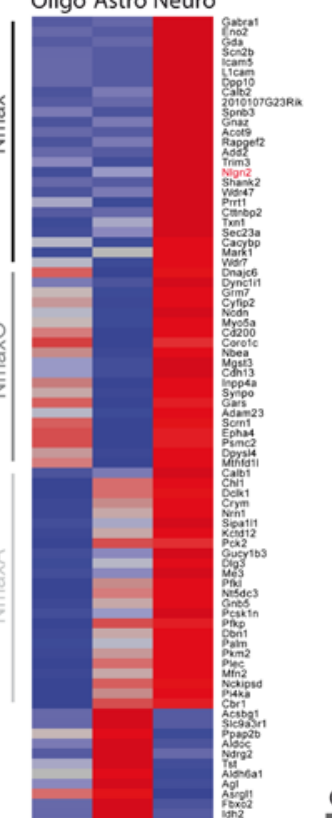

Oligo Astro Neuro

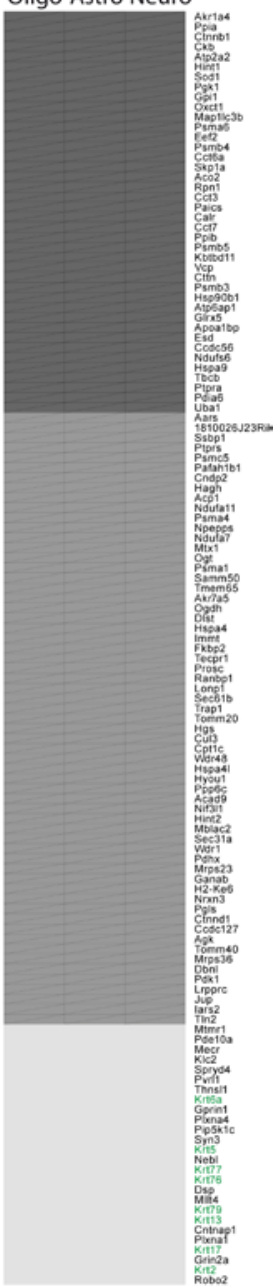

Figure 25: Clustering of proteins according to the corresponding mRNA expression in oligodendrocytes, astrocytes and neurons 
Following the quantification of the proteome data using spectral counting, proteins that were 2 -fold or more enriched in the sorted VGLUT1 ${ }^{\text {VENUS }}$ synaptosomes were grouped in the enriched group (163 proteins), while proteins depleted by a factor of 2 or more were grouped in the depleted group (343 proteins). For 156 of 163 proteins in the enriched group and for 334 of 343 proteins in the depleted group, cell type specific mRNA expression data from a study by Cahoy et al 2008 were available (Cahoy et al., 2008). For the proteins in each group the corresponding mRNA expression levels in oligodendrocytes, astrocytes and neurons were compared. Next the proteins were clustered according to their pattern of expression in theses cell types and the expression differences displayed as a heatmap. (A) Flow chart indicating each step of the transcriptome comparison and hierarchical clustering of proteins according to their corresponding mRNA expression. (B) Heatmap showing the differential gene expression of proteins in the enriched group and the depleted group. Clusters with maximal expression in neurons are indicated by labels left of the heatmap (see Table 3 for quantification). Note that the enriched group contains a high proportion of genes, that are selectively expressed in neurons and only few glial genes. In contrast, the depleted group contains only a low fraction of neuronal specific genes, among which are for example the genes of Neuroligin2 (NLGN2), GABA-receptor A1 (Gabra1) and Calretinin (Calb2), which are inhibitory markers. Gene symbols corresponding to proteins, that were also included in Western-blot analysis of FASS are colored in red. Gene symbols of proteins chosen as candidates for further studies are marked by a red dot. To illustrate differences in the genes that are not differentially expressed these were ranked according to their average mRNA expression. Notably, the strongly but evenly expressed group contains for example actin, that is known to be rich at synapses. In the depleted group, proteins that are weakly or not expressed include for example keratins, which are usual contaminants in proteomic experiments.

\section{Table 3: Summary of clustering of genes into different groups}

Summary of clustering of the enriched and depleted proteins according to their corresponding mRNA expression in neurons, astrocytes and oligodendrocytes as determined by Cahoy et al 2008 (see Figure 25).

Genes with differential expression

\begin{tabular}{lcccc} 
Group & $\begin{array}{c}\text { Enriched } \\
(\mathbf{9 8 / 1 5 6 )}\end{array}$ & Enriched (\%) & $\begin{array}{c}\text { Depleted } \\
(\mathbf{1 9 0 / 3 3 4 )}\end{array}$ & Depleted (\%) \\
\hline \hline Nmax & 41 & 41.84 & 24 & 12.63 \\
\hline NmaxO & 14 & 14.29 & 11 & 5.79 \\
\hline NmaxA & 13 & 13.27 & 23 & 12.11 \\
\hline Amax & 2 & 2.04 & 44 & 23.16 \\
\hline AmaxN & 5 & 5.10 & 28 & 14.74 \\
\hline AmaxO & 3 & 3.06 & 10 & 5.26 \\
\hline OmaxA & 3 & 3.06 & 7 & 3.68 \\
\hline OmaxN & 12 & 12.24 & 19 & 10.00 \\
\hline Omax & 5 & 5.10 & 24 & 12.63 \\
\hline differential & 98 & 100 & 190 & 100 \\
\hline \hline
\end{tabular}

Genes with uniform expression

\begin{tabular}{lcccc} 
Group & $\begin{array}{c}\text { Enriched } \\
(\mathbf{5 8 / 1 5 6 )}\end{array}$ & Enriched (\%) & $\begin{array}{c}\text { Depleted } \\
(\mathbf{1 4 4 / 3 3 4 )}\end{array}$ & Depleted (\%) \\
\hline \hline strong & 18 & 31.03 & 39 & 27.08 \\
\hline medium & 28 & 48.28 & 60 & 41.67 \\
\hline weak & 12 & 20.69 & 45 & 31.25 \\
\hline uniform & 58 & 100 & 144 & 100 \\
\hline \hline
\end{tabular}




\subsubsection{Clustering according to cell type specific gene expression data provides a global validation for the FASS procedure}

Following the quantification of our proteome data using spectral counting, proteins which were 2-fold or more enriched in the sorted VGLUT1 ${ }^{\text {VENUS }}$ synaptosomes were grouped in the enriched group (163 proteins), while proteins depleted by factor of 2 or more were grouped in the depleted group (343 proteins). For 156 of 163 proteins in the enriched group and for 334 of 343 proteins in the depleted group, cell type specific mRNA expression data from a study by Cahoy et al 2008 was available (Cahoy et al., 2008). For the proteins in each group the corresponding mRNA expression levels in oligodendrocytes, astrocytes and neurons were compared. Next the proteins were clustered according to their pattern of expression in theses cell types and the expression differences displayed as a heatmap. The flow chart in Figure 25A describes the algorithm used here. First, it was determined if genes are differentially expressed between cell types. If genes were not differentially expressed they were divided into three groups according to the level of expression (strong, medium or weak). The genes with differential expression were clustered according to the pattern of expression. It was first determined which cell types shows the maximal expression and then genes were grouped according to the relation of the expression in the remaining two cell types. Finally the relative expression of the genes was color coded from red (higher) over white to blue (lower). In this way heatmaps of differential expression in astrocytes, oligodendrocytes and neurons were generated (Figure 25). Table 3 summarizes the distribution of genes into the separate clusters.

There is a clear difference in the relative contribution of the different groups in the enriched and the depleted group. $69.4 \%$ of genes differentially expressed in the enriched group show maximal expression in neurons (Nmax, NmaxO, NmaxA) of which $60 \%$ (41.84 $\%$ total) show a relatively high expression only in neurons (Nmax). $10.2 \%$ of genes show maximal expression in astrocytes (Amax,AmaxO,AmaxN) $50 \%$ of which (5.1\% total) also show relatively high expression in neurons (AmaxN). $20.4 \%$ of genes show maximal expression in oligodendrocytes (Omax,OmaxA,OmaxN) of which $60 \%$ (12.24 \% total) also show relatively high expression in neurons (OmaxN).

In contrast, in the depleted group $30.53 \%$ of genes show maximal expression in neurons of which $41.3 \%$ (12.63\% total) are grouped in Nmax. At the same time, the depleted group contains a large fraction of genes with maximal expression in astrocytes $43.16 \%$ of which only $34 \%$ are grouped in AmaxN. The genes with maximal expression in oligodendrocytes contribute $26.31 \%$ of total. The OmaxN group contributes $38 \%$ of these (10\% total).

To summarize, the enriched group contains a much larger fraction of genes with specific or relative high expression in neurons, while the depleted group contains a much 
larger fraction of glia specific genes. In the enriched group the genes with maximal expression in neurons contain many known synaptic proteins like for example: SNAP25, Synaptophysin (Syp), Syntaxin1 (Stx1a), VGLUT1 (Slc17a7), CAPS1 (Cadps), Munc13-1 (Unc13a), EndophilinA1 (Sh3gl2), SV2B to name only a few. Notably, SV2B is expressed specifically in neurons, while SV2B is grouped in the OmaxN group, indicating a broader expression of SV2B. Notably in the enriched group the cluster of genes that is uniformly, but strongly expressed contains actin (Actb), which is known to be rich at synapses. Furthermore the depleted, but neuron specific genes include for example genes of Neuroligin2 (NLGN2), GABA-receptor A1 (Gabra1) and Calretinin (Calb2), which are known markers of inhibitory neurons. In the depleted group, proteins that are weakly or not expressed include for example keratins, which are usual contaminants in proteomic experiments.

\subsubsection{Five candidate proteins were chosen on the basis of enrichment in spectral counting, transcriptome comparison and literature research}

As outlined above, the quantitative comparison of VGLUT1 ${ }^{\text {VENUS }}$ synaptosomes isolated by FASS with gradient purified synaptosomes allowed the identification of 163 proteins, that were enriched by a factor of two or more in the sorted VGLUT1 ${ }^{\text {VENUS }}$ synaptosomes. Among these proteins were many with known synaptic functions, and the enrichment of several proteins was confirmed by Western blotting. However, apart from proteins with well-known functions at the synapse, there were also many proteins enriched in VGLUT1 $^{\text {VENUS }}$ synaptosomes for which no specific synaptic localization of function has been described to date. I therefore selected several proteins for further analysis in order to investigate their potential localization to VGLUT1 synapses. To add further confidence in my selection of protein candidates, I used the cell-type specific gene expression data of Cahoy et al. 2008 to select only those of the 163 enriched proteins, that showed a high neurons differential gene expression in neurons (see Nmax,NmaxO,NmaxA in Figure 25). From this subset I selected 4 protein candidates based on literature analysis and the availability of antibodies. The selected proteins were, FXYD6, Ly6H, Cavin1 and Cavin2. A fifth candidate protein I choose is TPD52. TPD52 was found to be enriched in VGLUT1 ${ }^{\text {VENUS }}$ synaptosomes, but its gene is also expressed in glial cell types. However, MAL2, a protein known to interact with TPD52, was recently identified as a specific component of VGLUT1 containing synaptic vesicles (Grønborg et al., 2010).

To study these candidate proteins further, I obtained antisera against TPD52, Ly6H, Cavin1 and Cavin2 (Abcam). Additionally, antibodies directed against mTPD52 and FXYD6 were provided by collaborating groups (Jennifer A. Byrne and Kathi Geering, respectively). Since the antibodies against Cavin1 and Cavin2 were not working reliably in initial test experiments, I focused my analysis on FXYD6, TPD52 and Ly6H (Figure 26). By applying a 
variety of approaches, I investigated if the chosen proteins are indeed localized to VGLUT1 synapses. For this purpose I prepared brain-region specific brain homogenates and subcellular fractionations, which were then analyzed by Western blotting. In addition, I performed Western blots or immunocytochemistry analysis on VGLUT1 ${ }^{\text {VENUS }}$ synaptosomes isolated by FASS. Finally $\mathrm{I}$ also studied immunofluorescence staining of cultured hippocampal neurons and sections of mouse brain. In this way, I provided first evidence indicating that FXYD6, TPD52 and Ly6H are associated with VGLUT1 synapses. (The immunofluorescence and immunogold-EM studies on FXYD6 were performed in collaboration with Mattia Aime, Véronique Bernard and Etienne Herzog at the department of Physiopathology of central nervous system diseases (INSERM UMR952) in Paris)

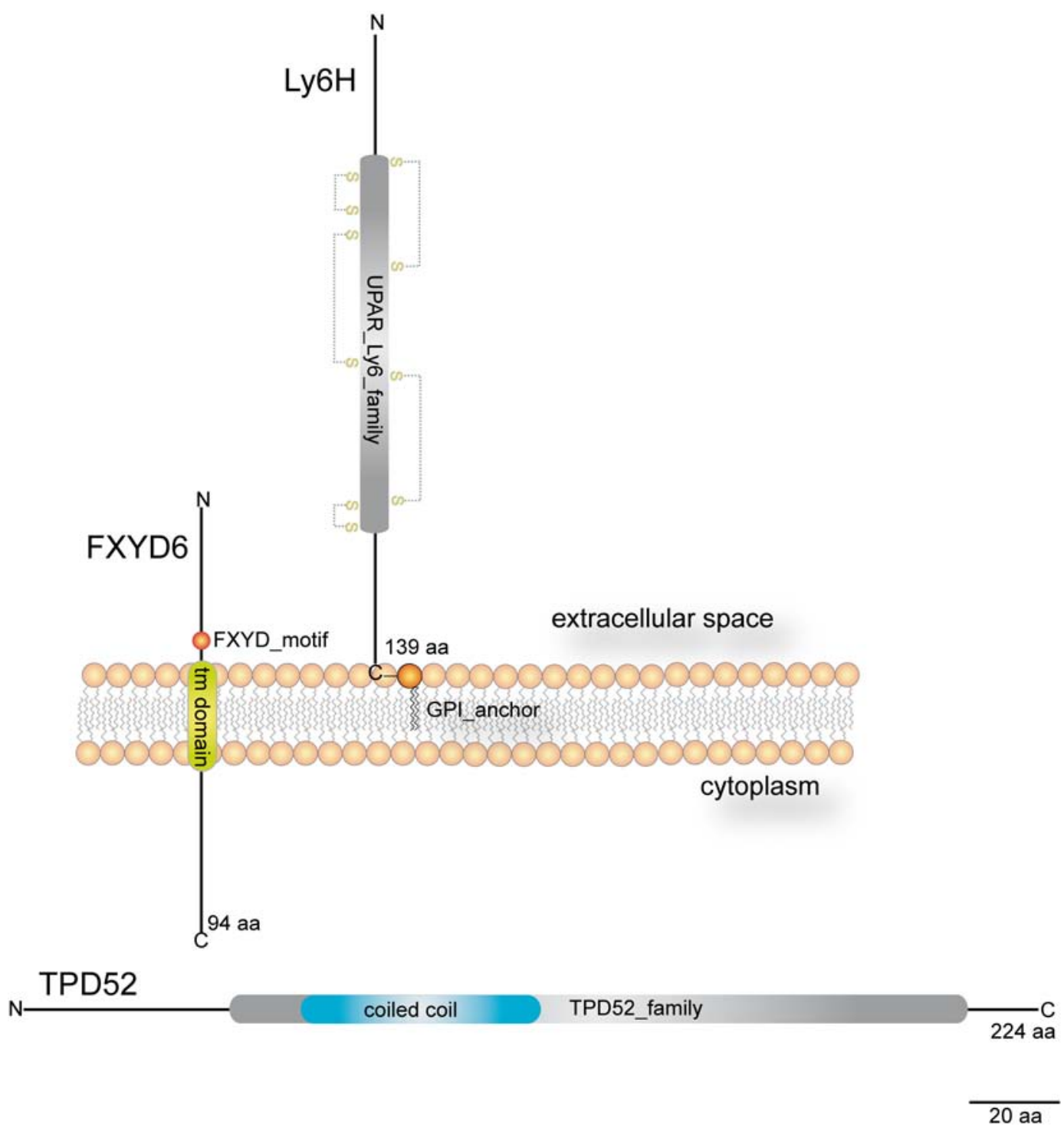

\section{Figure 26: Protein Candidates schematic representation}

Proteins are schematically drawn to scale and their domain structure is indicated. Tumor protein D52 (TPD52) is believed to be a cytosolic protein of 224 amino acids (aa). It contains a coiled coil domain and is the founding member of the TPD52 protein family. FXYD6 is a type-I transmembrane protein of 94 aa, the FXYD signature is comprised of the transmembrane domain and the FXYD-motif, which is extracellular. $\mathrm{Ly} 6 \mathrm{H}$, a protein of 139 
aa, is believed to be a secreted GPI anchored protein. Ly6H contains the Ly6-UPAR domain, which is characterized by a defined spacing and pattern of disulfide bonds.

\subsection{FXYD6}

FXYD6 was hardly detectable in the unsorted sample (normalized spectral count of 2.32, based on $1 \mathrm{HQ}$ peptide), while it was detected robustly in the sorted sample (normalized spectral count 4.39, based on $2 \mathrm{HQ}$ peptides) (Table 2). The spectral counts for this protein quantification were low, but the neuron specific expression revealed by the transcriptome analysis added further confidence in the selection of this protein for further analysis. Additionally, previous studies showed that FXYD6 expression is highly specific to the brain and that the protein can be localized to the membrane of neuronal somata and dendrites in the cortex, hippocampus and cerebellum (Kadowaki et al., 2004; Yamaguchi et al., 2001). A localization to synapses has not been reported so far. Therefore we investigated FXYD6 protein expression and localization in the mouse brain.

\subsubsection{FXYD6 is expressed throughout the brain and enriches in synaptic subcellular fractions}

FXYD6 was consistently enriched in sorted VGLUT1 ${ }^{\text {VENUS }}$ synaptosomes after FASS. The enrichment ratio of FXYD6 was 0.97, meaning that it was enriched to a similar extent as VGLUT1 $^{\text {VENUS }}$ itself (Figure $27 \mathrm{~A}$ ). Subcellular fractionation experiments revealed that FXYD6 is strongly enriched in the synaptic plasma membrane fraction (LP1B) (Figure 27 B). In addition, the protein was slightly enriched in the somato/dendritic endomembrane fraction (P3), as well as in the crude synaptic vesicle fraction (LP2). FXYD6 was detected in the soluble fractions S3 and LS2. FXYD6 was detected in all brain-region specific tissue homogenates analyzed (Figure $27 \mathrm{C}$ ). The strongest expression was seen in the forebrain, including cortex, caude-putamen and hippocampus. 
A

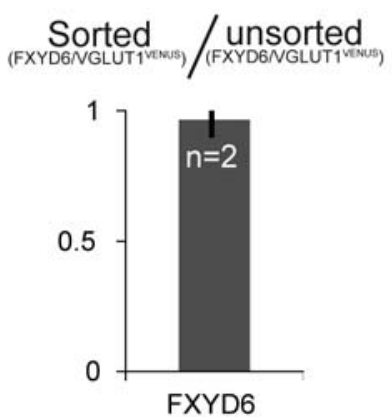

Synaptosomes FASS

$\begin{array}{llll}0.25 & 0.5 & 1.0 & \sim 0.5\end{array}$

B

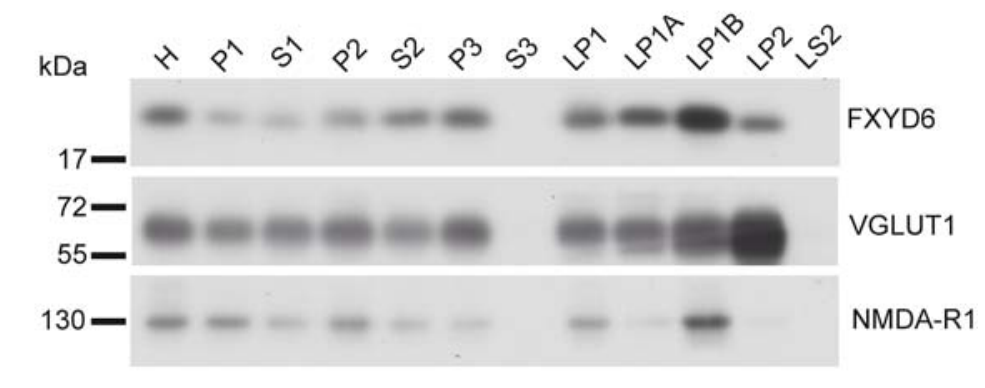

C

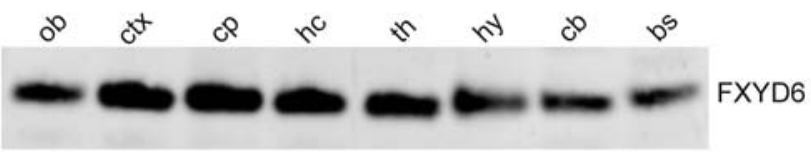

Figure 27: FXYD6 in FASS, subcellular fractions and different brain regions

Analysis of FXYD6 protein expression by Western blotting. (A) FXYD6 was analyzed for coenrichment with VGLUT1 in FASS (see Figure 19 for comparison) (B) Subcellular fractionation experiment analyzed for distribution of FXYD6, VGLUT1 and NMDA-R1. H: homogenate, P1: nuclear pellet, S1: Supernatant1, P2: crude synaptosomes, S2: Supernatant 2, P3: microsomal fraction, S3: somatic soluble fraction, LP1: lysed P2- Pellet1, LP1A:myelin rich - light membrane fraction, LP1B: synaptic plasma membrane fraction, LP2: crude synaptic vesicles, LS2: soluble synaptic fraction. (C) Western blot detection of FXYD6 in brain region specific tissue homogenates. Ob: olfactory bulb, ctx: cerebral cortex, cp: caude-putamen, hc: hippocampus, th: thalamus, hy: hypothalamus, cb: cerebellum, bs: brainstem. (brain region Western blot by M. Aime and Etienne Herzog).

\subsubsection{FXYD6 is neuron specific, localizes to the soma, dendrites and synapses hippocampal neurons}

In cultured hippocampal neurons prepared from VGLUT1 ${ }^{\text {VENUS }}$ knock-in mice, FXYD6 displayed a somato/dendritic expression pattern. In addition to the somato/dendritic localization, FXYD6 was frequently detected as intense punctate staining which was partially colocalized with or in direct apposition to VGLUT1 ${ }^{\text {VENUS }}$ puncta (Figure $28 \mathrm{~A}$ ). In hippocampal neurons prepared from wild type animals, FXYD6 showed a similar expression pattern. I next performed triple-immunofluorescence stainings for FXYD6, VGLUT1 (pre-synapse) and PSD95 (postsynaptic density). Even though a large proportion of FXYD6 was localized to the somata and dendrites, intense FXYD6 synaptic puncta were partially colocalized with VGLUT1, but not with PSD95 (see arrowheads). Careful examination of the images showed that in addition to the presynaptic FXYD6 there was also a weaker signal for FXYD6 in the postsynaptic areas. A co-staining with MAP2, which marks the microtubules in the centre of dendrites, showed that the dendritic FXYD6 is mostly localized to the periphery (plasma membrane) of the dendrite. The hippocampus neuron cultures also contain a small fraction of VGLUT2 expressing neurons and synapses. The punctate FXYD6 staining was also partially co-localized with VGLUT2 puncta (Figure 28 B). FXYD6 was not or very weakly detected in hippocampal astrocytes expressing GFAP (Figure $28 \mathrm{~B}$ ). 
A

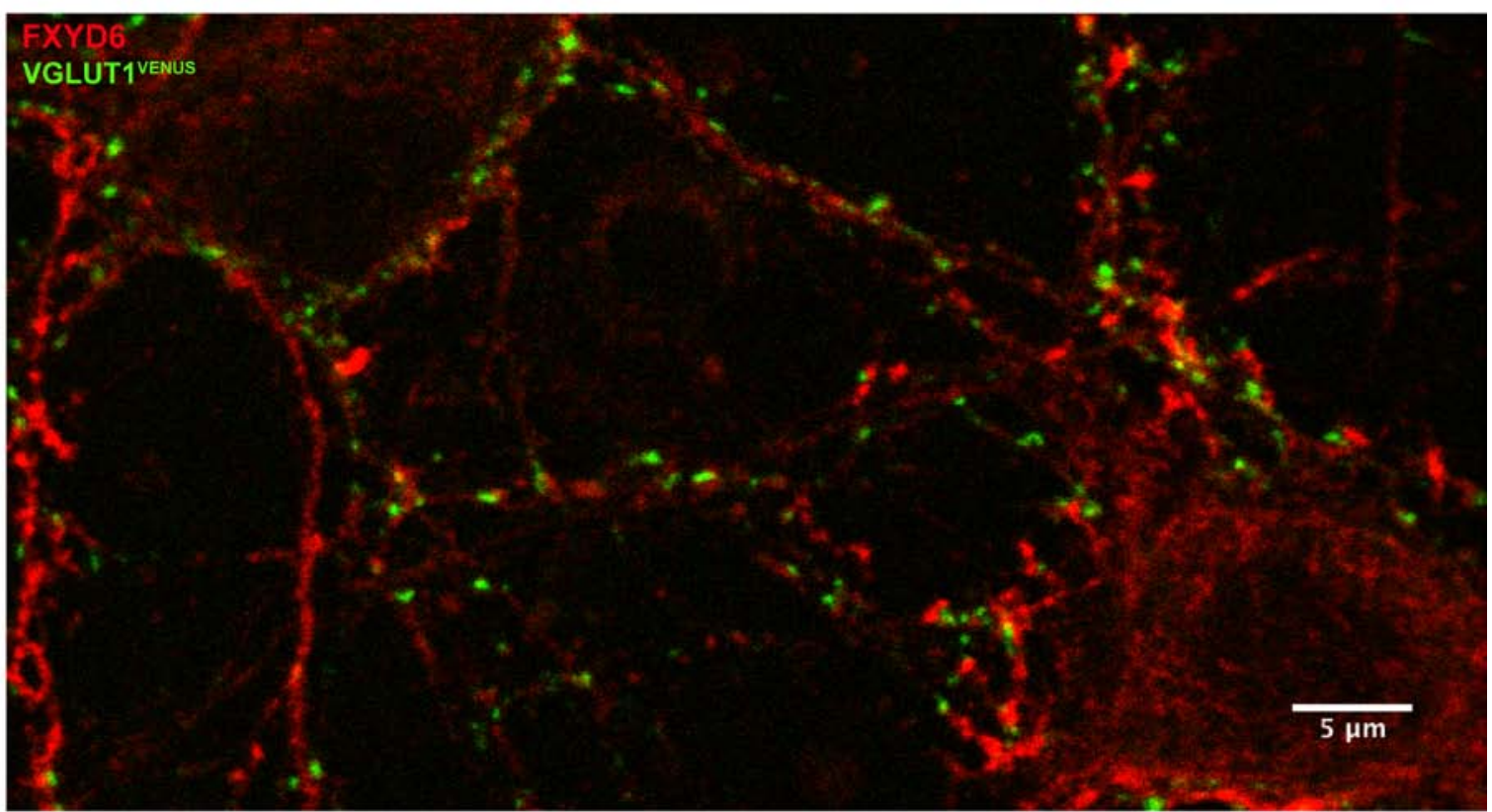

B
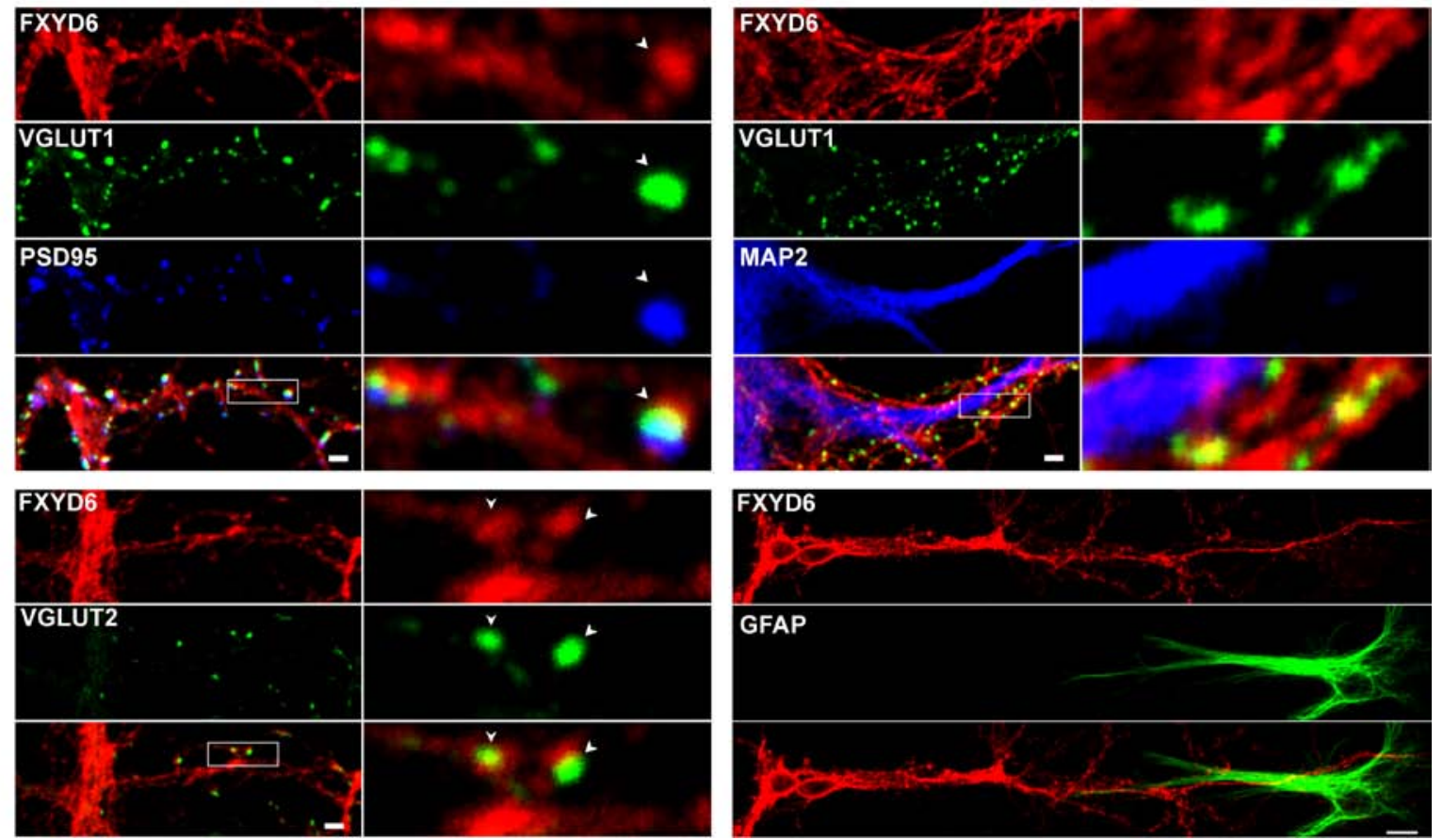

Figure 28: FXYD6 in primary hippocampal neuron cultures

Immunofluorescence colocalization in primary hippocampal neuron cultures. (A) FXYD6 immunofluorescence in cultures of VGLUT1 ${ }^{\text {VENUS }}$ knock-in mice. The endogenous VGLUT1 ${ }^{\mathrm{VENUS}}$ fluorescence is shown in green and FXYD6 immunofluorescence in red. Note the close apposition of VGLUT1 ${ }^{\text {VENUS }}$ and FXYD6 puncta. (B) Costaining for FXYD6 (red) and several marker proteins in wild type neurons. Triple-labeling with VGLUT1 and PSD-95 indicates the relative position of FXYD6 to pre/post-synaptic elements and co-expression with VGLUT1. Triple labeling with VGLUT1 and MAP2 indicates relative position of FXYD6 to the presynapse and the dendritic centre. Costaining with VGLUT2 or GFAP analyzes expression in VGLUT2 expressing neurons/synapses and astrocytes respectively. Scale bar, $10 \mu \mathrm{m},(2 \mu \mathrm{m}$ in enlarged regions) (images M. Aime and E. Herzog) 


\subsubsection{FXYD6 localizes to the soma, dendrites and synapses of neurons in situ}

In the mouse cerebral cortex, FXYD6 displayed an intense fibrous staining pattern (Figure $29 \mathrm{~A}$ ). In addition to the fiber staining, there was also a punctate staining of lower intensity in synapse rich regions. In co-stainings with VGLUT1 and FXYD6, a partial colocalization or close apposition of FXYD6 puncta with VGLUT1 puncta was observed (see enlarged images in Figure $29 \mathrm{~A}$ ). The peripheral staining in the dendrites that was observed in hippocampal cultures was also observed in co-stainings of FXYD6, MAP2 and VGLUT1 in the hippocampus CA3 region (Figure 29 B). Co-staining of the inhibitory synapse specific markers GAD65/67 with FXYD6 showed that varicosities positive for GAD65/67 were largely devoid of FXYD6 signal. Overall the two markers displayed a segregated pattern in this region. On the other hand, FXYD6 signals were present in the periphery of cell bodies of inhibitory neurons in the CA1 region (Figure 29 B). Pre-embedding, silver-enhanced immunogold labeling of FXYD6 for electron microscopy showed a frequent labeling close to the plasma membrane of boutons and surrounding axonal membrane in CA3 (Figure 29 C). Only occasionally an accumulation of signals in the vicinity of vesicle clusters within these boutons was observed. 
A

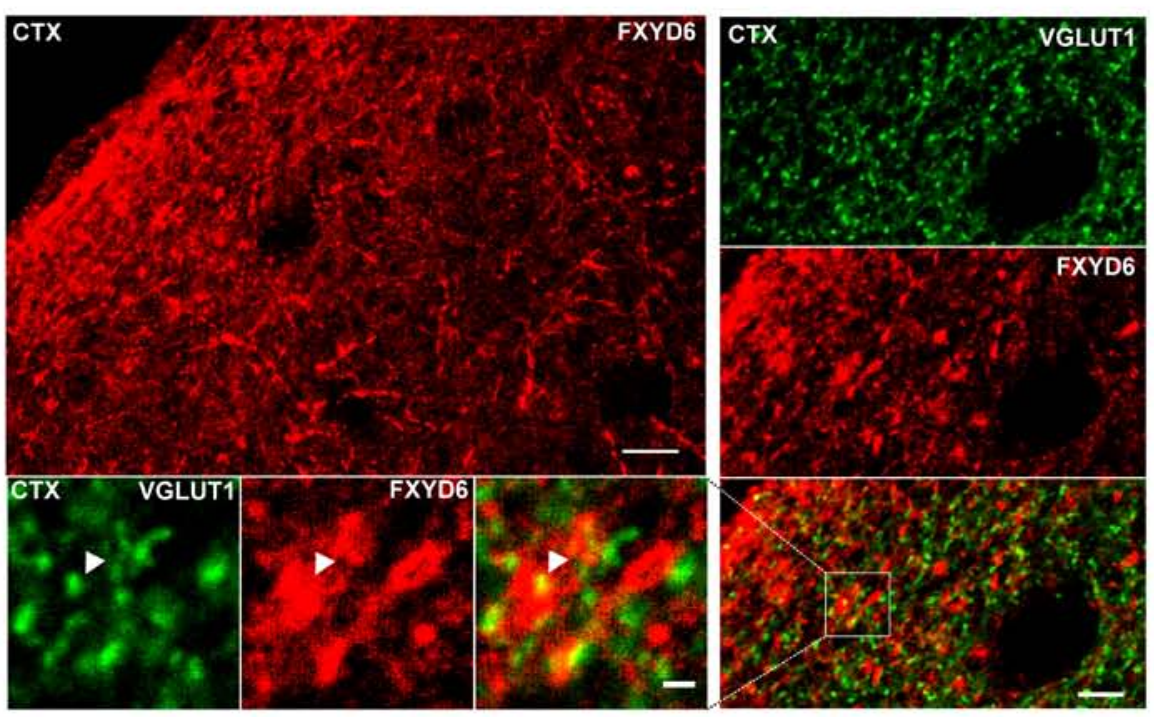

B

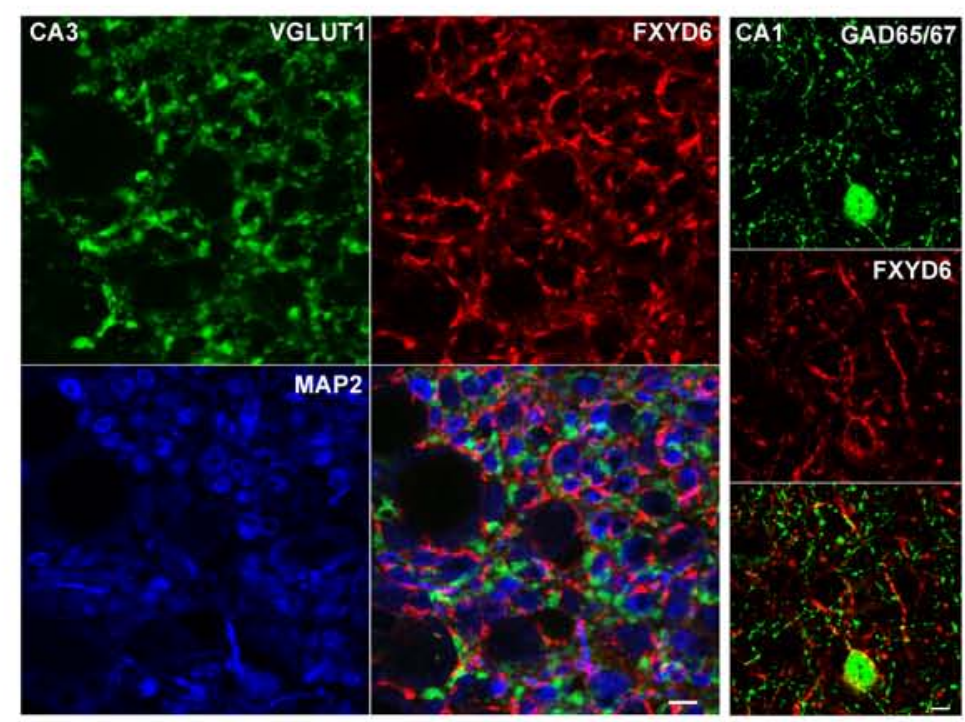

C

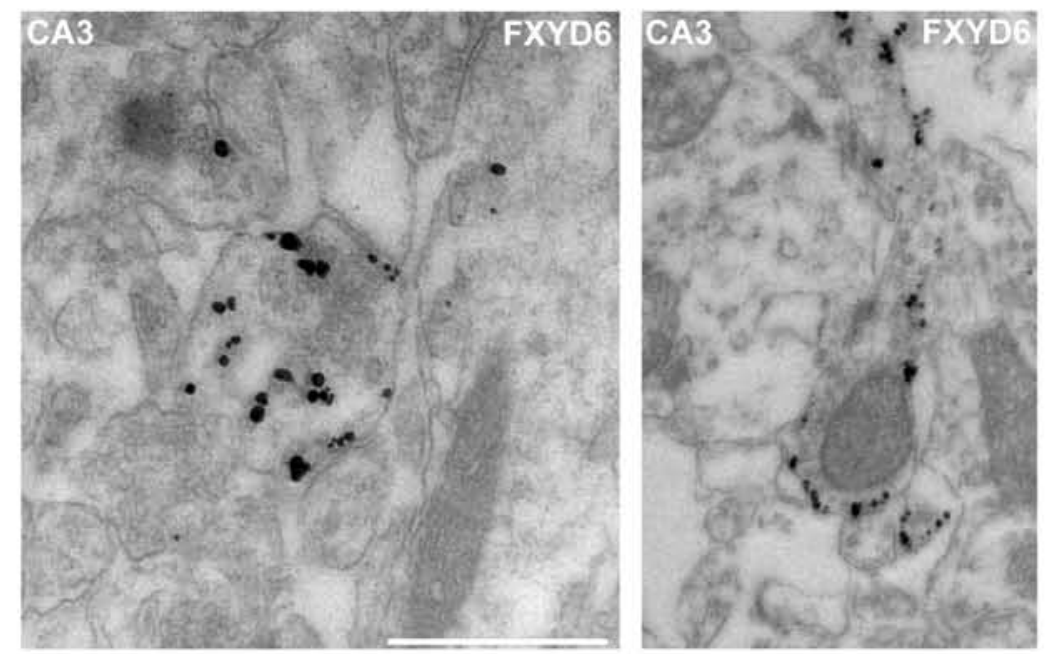




\section{Figure 29: FXYD6 in sections}

Immunostainings of FXYD6 in sections of the mouse brain. (A) Labeling of FXYD6 and VGLUT1 in the cerebral cortex, note the frequent close apposition of VGLUT1 and FXYD6 as well as partial colocalization (arrowhead) Scale bars, $10 \mu \mathrm{m}, 5 \mu \mathrm{m}$ and $1 \mu \mathrm{m}$ (B) Tripleimmunostaining in the hippocampus CA3 region for VGLUT1, MAP2 and FXYD6 and doubleimmunostaining for FXYD6 and GAD65/67 in hippocampal CA1.scale bar, $5 \mu \mathrm{m}$ (C) Postembedding, silver-enhanced immunogold labeling of FXYD6 in hippocampal CA3. Scale bar is $1 \mu \mathrm{m}$ for the left, and $2 \mu \mathrm{m}$ for the right image (Images provided by M. Aime, E. Herzog and V. Bernard)

\section{$3.9 \quad$ TPD52}

The comparative analysis of unsorted synaptosomes with VGLUT1 ${ }^{\text {VENUS }}$ synaptosomes isolated by FASS indicated a 3.33-fold enrichment for TPD52 in the VGLUT1 ${ }^{\text {VENUS }}$ synaptosomes as analyzed by spectral counting. In the unsorted sample TPD52 had a normalized spectral count of 3.42 based on 2 HQ-peptides (see Table 2). In the sorted VGLUT1 ${ }^{\text {VENUS }}$ synaptosomes TPD52 had a spectral count of 10.54 , based on 7 HQ-peptides. Additionally, TPD52 is known to interact with MAL2, a protein that was recently shown to be a specific component of VGLUT1 containing synaptic vesicles (Grønborg et al., 2010), and TPD52 family proteins have mainly been associated with vesicle trafficking and secretory processes (for review see (Boutros et al., 2004)). Taken, together this information leads to the interesting possibility that TPD52 might localize to VGLUT1 synapses and function in synaptic vesicle trafficking. To test this hypothesis I investigated the protein expression and localization of TPD52.

\subsubsection{TPD52 is expressed in many brain regions and localizes to VGLUT1 pre- and postsynapses}

In Western blots of brain-region specific tissue homogenates TPD52 showed the strongest expression in the olfactory bulb, but was also detected robustly in cortex, caudeputamen, hippocampus, brainstem and spinal cord (Figure 30). The midbrain and cerebellum showed only weak TPD52 expression. Upon subcellular fractionation of forebrain tissue, TPD52 was enriched in the soluble synaptic fraction (LS2). In addition, the protein was also detectable in the somato-dendritic microsome and soluble fractions (P3 and S3). There was no detectable signal for TPD52 in the crude synaptic vesicle fraction. Interestingly, in the myelin rich LP1A fraction, a protein-band of higher molecular weight is also revealed by the TPD52 antibody. 
A

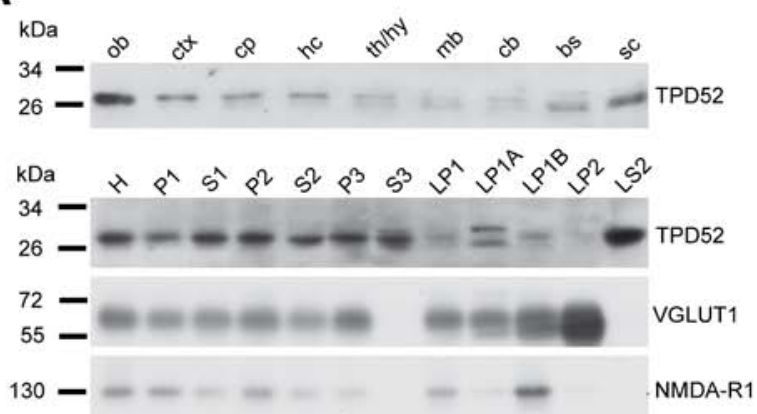

C
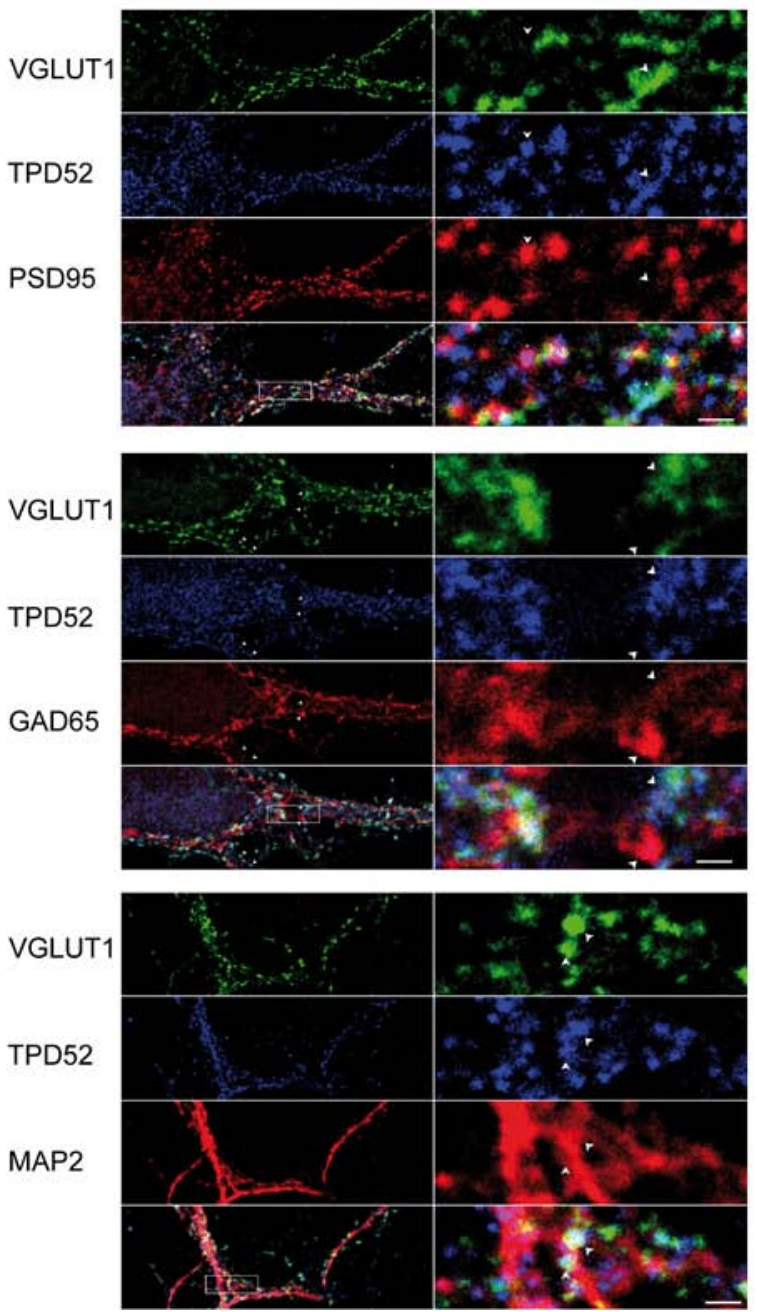

B

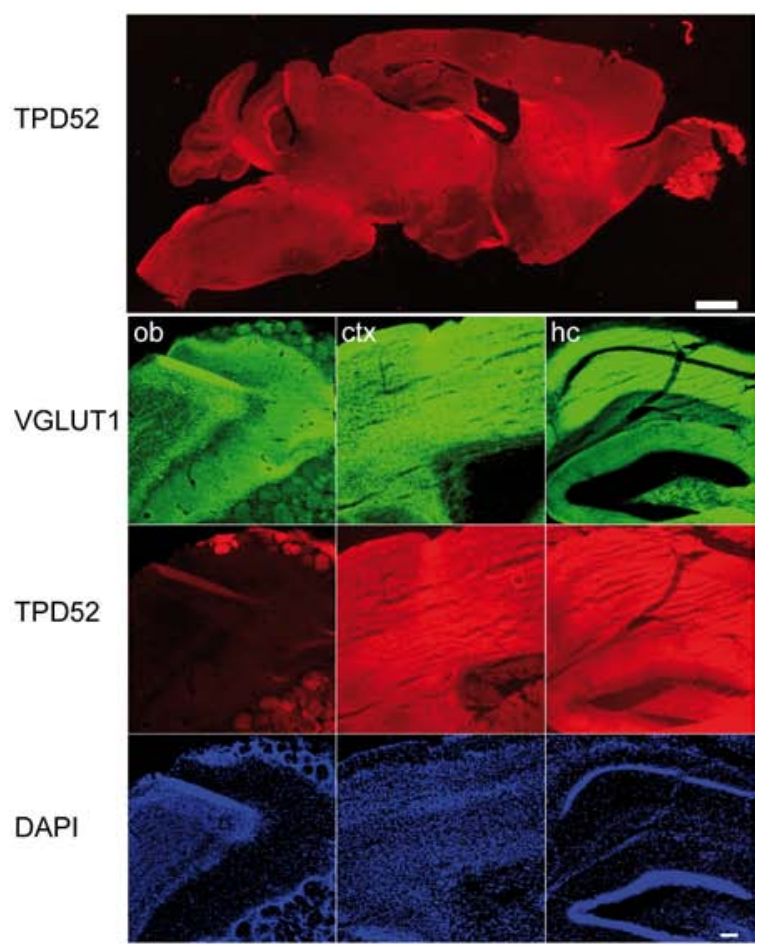

D

GFP

TPD52

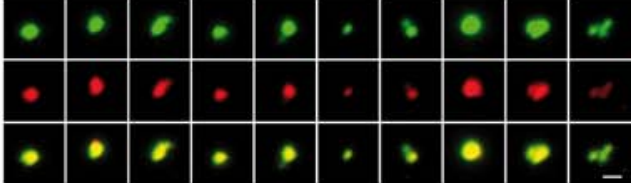

quantification of

VGLUT1 1ENUS/TPD52 synaptosomes

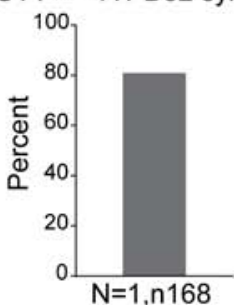

\section{Figure 30: TPD52 localization at VGLUT1 synapses}

Analysis of TPD52 protein expression and localization in different subcellular fractionations, brain sections, primary hippocampal neurons and VGLUT1 ${ }^{\text {VENUS }}$ synaptosomes after FASS. (A) (upper part) Western blot analysis of TPD52 expression in brain region specific tissue homogenates. Ob: olfactory bulb, ctx: cerebral cortex, cp: caude-putamen, hc: hippocampus, th/hy: thalamus, hypothalamus, cb: cerebellum, bs: brainstem, sc: spinal cord. (lower part) Subcellular fractionation experiment analyzed for distribution of TPD52, VGLUT1 and NMDAR1. H: homogenate, P1: nuclear pellet, S1: Supernatant1, P2: crude synaptosomes, S2: Supernatant 2, P3: microsomal fraction, S3: somatic soluble fraction, LP1: lysed P2- Pellet1, LP1A: myelin rich - light membrane fraction, LP1B: synaptic plasma membrane fraction, LP2: crude synaptic vesicles, LS2: soluble synaptic fraction. (B) Immunofluorescence staining for TPD52 and VGLUT1 in sections of the mouse brain. (top) Overview showing the overall staining pattern for TPD52. (bottom) higher magnification images showing the 
staining for TPD52, VGLUT1 and DAPI in the olfactory bulb (ob), cerebral cortex (ctx) and hippocampus (hc). The exposure time of images of TPD52 immunofluorescence in the olfactory bulb was lower than in the other two brain regions in order not to saturate the signal. Scale bar, $2 \mu \mathrm{m}$ in higher magnifications (C) Triple immunofluorescence stainings for TPD52 (blue), VGLUT1 (green) and either PSD95, GAD65 or MAP2 (all in red) in cultured primary hippocampal neurons (DIV22). (D) Immunofluorescence staining for VGLUT1 ${ }^{\text {VENUS }}$ (GFP antibody) and TPD52 in VGLUT1 ${ }^{\text {VENUS }}$ positive synaptosomes after FASS. The number of double positive particles was quantified.

In cultured hippocampal neurons, immunofluorescence staining for TPD52 shows a punctate somatic staining in addition to a punctate staining in the dendritic regions. TPD52 was colocalized with both VGLUT1 and PSD95 in many instances. In confocal microscope images of triple-stainings for TPD52, VGLUT1 and GAD65, TPD52 was either colocalized with VGLUT1 or with VGLUT1 and GAD65, but never with GAD65 alone. Co-staining for MAP2 showed that the TPD52 puncta are usually localized in the vicinity, but not in the center of the dendrites. In a quantitative analysis of epifluorescence images immunofluorescence stainings of sorted VGLUT1 ${ }^{\text {VENUS }}$ synaptosomes, 80 percent of VGLUT1 $^{\text {VENUS }}$ positive synaptosomes were also positive for TPD52 (Figure 30 D).

\section{$3.10 \mathrm{Ly} 6 \mathrm{H}$}

Ly6H is a member of the u-PAR-Ly6 protein family (Ly6 family), which consists of several small proteins (12-20kDa) with a conserved sequence of 8-10 cysteines that are characterized by a specific pattern of disulfide bonds (Ploug and Ellis, 1994; Ploug et al., 1993) (Figure 26). Proteins of the Ly6 family can be intracellular or secreted and tethered to the cell surface via a glycosylphophatidylinositol (GPI)-anchor. Ly6H mRNA and protein expression was found in several brain regions, but $\mathrm{Ly} 6 \mathrm{H}$ is not expressed in the cerebellum (Apostolopoulos et al., 1999; Dessaud et al., 2006; Horie et al., 1998).

My comparative analysis of unsorted synaptosomes and VGLUT1 ${ }^{\text {VENUS }}$ synaptosomes isolated by FASS indicated a 5-fold enrichment of $\mathrm{Ly} 6 \mathrm{H}$ in the sorted VGLUT1 ${ }^{\text {VENUS }}$ synaptosomes. In the unsorted synaptosomes the normalized spectral count of Ly6H was 3.48 (2 HQ peptides), while in the sorted sample the spectral count was 20.21 (5 HQ peptides) (see Table 2). Furthermore, Ly6H is preferentially expressed in neurons as indicated by the transcriptome data of Cahoy et al. (2008) (Figure 25). Together this information indicates that Ly6H localizes to VGLUT1 synapses. I therefore investigated the localization of Ly6H to VGLUT1 synapses by various methods. 


\subsubsection{Ly6H at VGLUT1 synapses}

In Western blots of brain-region specific tissue homogenates the $\mathrm{Ly} 6 \mathrm{H}$ antibody recognized several protein-species at different apparent molecular weights. Notably, a protein of apparent molecular weight of $20 \mathrm{kDa}$ is recognized in the tissue homogenates of many brain regions including cortex, hippocampus, caude putamen and thalamus/hypothalamus, but not in cerebellum. The theoretical molecular weight of $\mathrm{Ly} 6 \mathrm{H}$ is $15 \mathrm{kDa}$ and Ly6H is very likely GPI-anchored (Apostolopoulos et al., 1999; Horie et al., 1998). Additionally, these studies showed that Ly6H is not or only very weakly expressed in cerebellum. Therefore we concluded that the protein recognized by the $\mathrm{Ly} 6 \mathrm{H}$ antibody at an apparent molecular weight of $20 \mathrm{kDa}$ is $\mathrm{Ly} 6 \mathrm{H}$. Interestingly, upon subcellular fractionation of forebrain tissue Ly6H was strongly enriched in synaptic plasma membrane fractions (LP1B) and to a lesser extend in crude synaptic vesicles. Immunofluorescence staining of brain sections with the same antibody as used in Western blotting showed staining for $\mathrm{Ly} 6 \mathrm{H}$ in synapse rich layers of several brain regions including cortex, caude putamen and hippocampus. No staining was detected in the granular cell layer or the molecular layer of the cerebellum. Western blot analysis of cultured primary cerebellar neurons confirmed that Ly6 $\mathrm{H}$ was not detected in these cells. Interestingly, in Western blots of cultured primary hippocampal neurons, Ly6H was detected with much less background from other proteins. In cultured primary hippocampal neurons Ly6H immunofluorescence staining displayed a punctate somato dendritic staining pattern. This punctate staining of Ly6H was frequently in close apposition to the stainings of VGLUT1 pre- and postsynaptic elements as indicated by costaining of Ly6H, VGLUT1 and ProSAP1 (Figure $31 \mathrm{C}$ ). Furthermore, quantification of immunofluorescence labeling of VGLUT1 ${ }^{\text {VENUS }}$ synaptosomes isolated by FASS in two independent experiments indicates that $60 \%$ of sorted VGLUT1 ${ }^{\text {VENUS }}$ synaptosomes are also positive for Ly6H. 
A

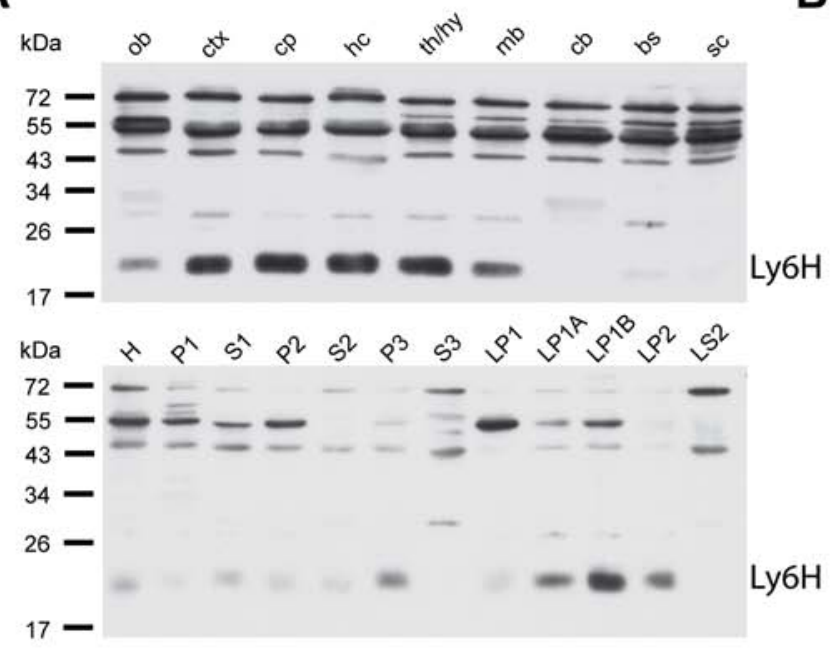

C

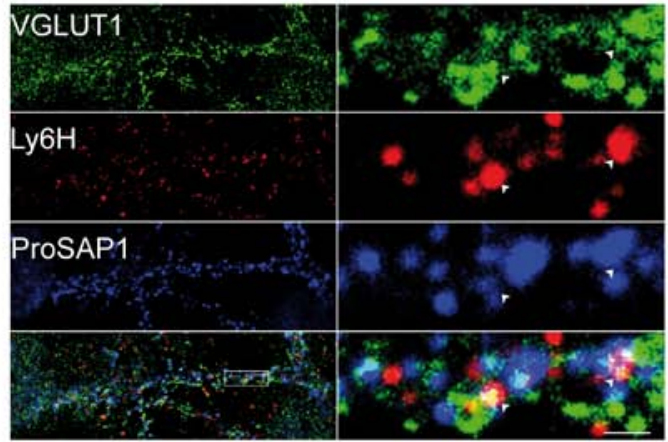

neuronal culture

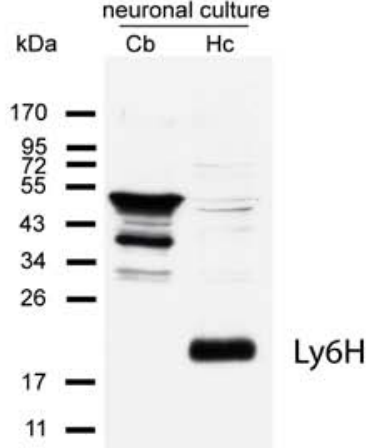

B
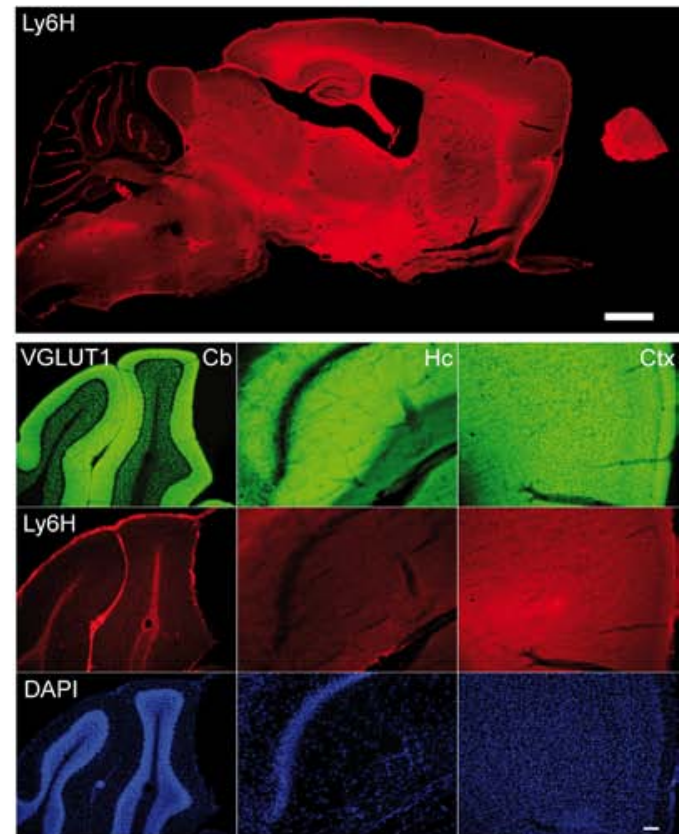

D
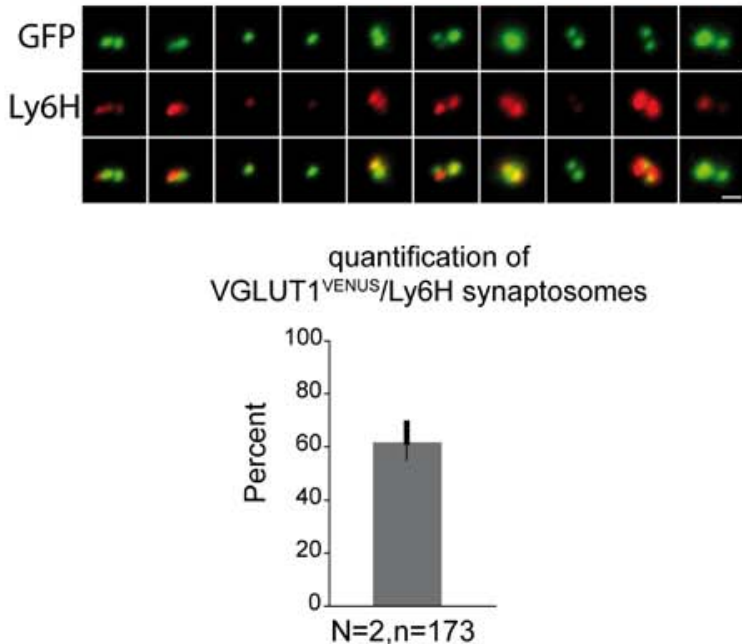

\section{Figure 31: Ly6H expression at VGLUT1 synapses}

Analysis of $\mathrm{Ly} 6 \mathrm{H}$ protein expression in different subcellular fractionations, brain sections, primary hippocampal neurons and VGLUT1 ${ }^{\text {VENUS }}$ synaptosomes after FASS. Note that previous studies demonstrated that $\mathrm{Ly} 6 \mathrm{H}$ is not expressed in the cerebellum. (A) (upper part) Western blot analysis of $\mathrm{Ly} 6 \mathrm{H}$ expression in brain region specific tissue homogenates. Note the absence of specific Ly6H signal in the cb. Ob: olfactory bulb, ctx: cerebral cortex, $\mathrm{cp}$ : caude-putamen, hc: hippocampus, th/hy: thalamus, hypothalamus, cb: cerebellum, bs: brainstem, sc: spinal cord. (lower part) Subcellular fractionation experiment analyzed for distribution of Ly6H, VGLUT1 and NMDA-R1. H: homogenate, P1: nuclear pellet, S1: Supernatant1, P2: crude synaptosomes, S2: Supernatant 2, P3: microsomal fraction, S3: somatic soluble fraction, LP1: Iysed P2- Pellet1, LP1A: myelin rich - light membrane fraction, LP1B: synaptic plasma membrane fraction, LP2: crude synaptic vesicles, LS2: soluble synaptic fraction. (B) Immunofluorescence staining of Ly6H and VGLUT1 in sections of the mouse brain. (top) overview showing the overall staining pattern for Ly6H. Note the difference in signal between the cerebellum and the forebrain. (bottom) Higher magnification images showing the staining for Ly6H, VGLUT1 and DAPI in the cerebellum (cb), cerebral 
cortex (ctx) and hippocampus (hc). (C) Triple immunofluorescence stainings of postsynaptic ProSAP1 (blue) with presynaptic VGLUT1 (green) and Ly6H (red) in primary cultured hippocampal neurons (DIV22). Scale: left $15 \mu \mathrm{m}$, right $2 \mu \mathrm{m}$. And Western blot of Ly6H in mixed cultures of the cerebellum and cultured hippocampal neurons. Note the difference in non-specific staining in the molecular weight range between 34 and $55 \mathrm{kDa}$ (D) Immunofluorescence staining of VGLUT1 $1^{\text {VENUS }}$ (GFP antibody) and Ly6H in VGLUT1 $1^{\text {VENUS }}$ positive synaptosomes after FASS. The number of double positive particles was quantified from two independent experiments. 


\section{Discussion}

In the present study, I have developed a new protocol for the purification of VGLUT1 specific synaptosomes by fluorescence activated synaptosome sorting (FASS) from VGLUT1 ${ }^{\text {VENUS }}$ knock-in mice. Using Western blotting, immunofluorescence microscopy and electron microscopy I validated this new protocol and demonstrated the superior purity of FASS-purified synaptosomes as compared to conventional synaptosome preparations. In addition, electron microscopy and immunofluorescence microscopy confirmed the structural integrity of synaptosomes after FASS. Using FASS, I addressed open questions on the cellular and subcellular localization of several synaptic proteins including Neuroligins and NMDA-receptor isoforms. Furthermore a proteome analysis provided an extensive catalogue of proteins contained in conventional synaptosomal preparations and in sorted VGLUT1 ${ }^{\text {VENUS }}$ synaptosomes. By means of spectral counting, I could identify a set of 163 proteins that are specifically enriched in this synaptosome subtype as compared to the total synaptosomal preparation. Several proteins of this set had not previously been detected at VGLUT1 synapses. For three of these proteins, namely FXYD6, TPD52 and Ly6H, I provide evidence for their VGLUT1-synaptic localization.

\subsection{Methodological developments allow the analysis of FASS samples by independent methods}

The characteristics of different cell types in flow cytometry, especially of blood cells, are known very well known. In fluorescence-activated-cell-sorting, the sorted cells can be reanalyzed for their purity by flow-cytometry and can be proliferated in cell culture to allow subsequent analyses by independent methods. In this study I used cytometry approaches within the context of a biochemical fractionation approach and therefore encounter several technological hurdles linked to the fact that synapses are an order of magnitude smaller than an average cell and that synaptosomes cannot be proliferated after purification.

Prior to the present study, the characteristics of synaptosomal preparations in flow cytometry had been studied only cursorily and methods for independent validation of measurements had been limited. Previously published studies employed flow cytometry to analyze the distribution and colocalization of different proteins within the synaptosome preparation by indirect immunofluorescence. Furthermore, the light-scattering distribution of synaptosomal particles was studied in relation to polystyrene microspheres of different diameter. These studies indicated that synaptosomes fall in a size range of $0.75-1.4 \mu \mathrm{m}$ (Gylys et al., 2004a; Wolf and Kapatos, 1989b). The identification of synaptosomes in these studies was based on the staining with vital dyes and the presence of neuronal or synaptic 
marker proteins, such as SNAP25. Wolf and collaborators also sorted a population of TH positive particles and analyzed the enrichment of TH using immunoblotting techniques (Wolf and Kapatos, 1989a; Wolf and Kapatos, 1989b). Although a 6-fold enrichment of TH was found upon immunoblotting, the enrichment or depletion of other markers was not examined. Gylys and colleagues have focused their work on analyzing, rather than sorting synaptosomes. In order to substantiate that the analyzed particles are in fact synaptosomes they also sorted synaptic particles and imaged them by electron microscopy (Fein et al., 2008). However, only negative staining of the particles, which were sorted directly onto an electron microscope grid, was performed. While this analysis revealed the presence of round membrane bounded particles of varying sizes around $0.5 \mu \mathrm{m}$, it did not allow to analyze the ultrastructure of the sorted particles. All previous studies of synaptosomes that were based on flow cytometry have reported extraordinarily high percentages of synaptic particles and surprisingly low contaminations by glial components in their synaptosomal preparations (see Introduction). Interestingly, none of the two groups reported the re-analysis of sorted samples by flow cytometry, although this could have provided a very quick and straightforward validation for their analyses. Taken together the identity of the particles that were analyzed and sorted by previous flow cytometric studies of synaptosomes is not known. Based on the findings in the present study it is unlikely that the particles analyzed and sorted in these studies were single synaptosomes and therefore also the purity of previous flow-cytometry based synaptosome isolations is questionable.

\subsubsection{Methods for the analysis of FASS samples}

In order to allow for a more thorough analysis of the synaptosomal particles analyzed and sorted in our FASS procedure, I have developed procedures that allow the analysis of sorted synaptosomal particles by Western blotting, immunofluorescence microscopy and electron microscopy. For the recovery of protein from highly diluted synaptosome suspensions for Western blotting, I obtained the best results by applying vacuum filtration onto polycarbonate filter membranes with a uniform pore size of $0.22 \mu \mathrm{m} .56 \%$ of the initial synaptosomal protein could be recovered from the filters using detergent containing solutions, but not with salt buffers, which precludes this method from recovering synaptosomes for functional analyses. However, an advantage of the use of the Isopore ${ }^{\mathrm{TM}}$ filters was that they allowed for fixation, embedding and sectioning of synaptosomes for electron microscopy.

It is possible that the synaptosomal preparation contains particles that are smaller than $0.22 \mu \mathrm{m}$. However, intact synaptosomes have been reported to have a diameter of at least $0.5 \mu \mathrm{m}$ and should therefore be retained by the Isopore ${ }^{\mathrm{TM}}$ filter. Accordingly the loss of $44 \%$ of total protein in re-concentration of unsorted synaptosomal preparation probably 
results from the combination of loss of particles through unspecific adsorption and the passage of smaller particles through the filter pores.

In order to further improve the sample recovery process for Western blotting and EM, a cooled, vacuum-filtration device, adapted to the FACSAria was developed. Using the developed online-filtration device, samples can be sorted and concentrated simultaneously, thereby reducing the total time of the procedure by several hours.

Following flow-sorting, the synaptosomes are in a highly diluted suspension. I demonstrated that using a centrifugation procedure enables the quantitative recovery of these synaptic particles onto gelatinized coverslips. This method therefore allowed me to study and quantify the colocalization of various markers in synaptic particles after FASS using immunofluorescence microscopy. In summary the methods developed in this study allow for the analysis of sorted synaptosomes at an unprecedented level of detail.

\subsection{FASS in FM-mode enriches intact, single VGLUT1 ${ }^{\text {VENUS }}$ synaptosomes}

Previously the light scattering of synaptosomes had been reported to be in between the signal of 0.75 and $1.5 \mu \mathrm{m}$ polystyrene microspheres (Gylys et al., 2004a; Wolf and Kapatos, 1989b). My results indicate that particles in this size range are not single synaptosomes, but rather correspond to aggregates. These findings are in conflict with the previous studies by Wolf and colleagues and Gylys and colleagues, but are supported by that fact that in the present study: (i) VGLUT1 ${ }^{\text {VENUS }}$ positive particles isolated in the size range of 0.75-1.5 $\mu \mathrm{m}$ were also enriched in VIAAT and PLP as analyzed by Western blotting. (ii) Staining with FM4-64 revealed that a large number of particles in the synaptosomal preparation fall in a size range below $0.75 \mu \mathrm{m}$ and VGLUT1VENUS positive particles were also present among these particles. (iii) Western blotting, immunofluorescence microscopy and electron microscopy all show that sorted VGLUT1 ${ }^{\text {VENUS }}$ positive particles that fell into the size range below $0.75 \mu \mathrm{m}$ represent intact single synaptosomes.

\subsubsection{Evidence from flow cytometry}

During flow analysis and sorting, particles are exposed to large pressure differentials and shearing forces that occur when the fluid stream that carries the particles exits the pressurized fluidics system through a $70 \mu \mathrm{m}$ size nozzle. If particles are unaltered by the passage through the sort-instrument one expects that they will present the same apparent light-scattering characteristics as unsorted particles. In my FSC-triggered experiments I observed a reduction in the fraction of large and fluorescently positive particles. This can be 
considered as an indication for the breakdown of pre-existing aggregates during the sort process. Several weakly fluorescent or autofluorescent particles would result in an overall positive fluorescence of an aggregate, which if broken down, generates a number of smaller non-fluorescent particles. In addition, the presence of a single positive synaptosome will render an aggregate of otherwise negative elements fluorescently positive. In line with our observation, breakdown of these aggregates results in a systematic reduction of particle sizes, but not of the overall fluorescence intensity.

Alternatively the reduction in large particles could result from the disintegration of single fluorescent synaptosomes by the same shearing forces and pressure differentials. Indeed I experienced this phenomenon when testing high pressure/speed sorting on an alternative instrument (MofloXDP from BeckmanCoulter, Data not shown). As a result I could see a major drop in fluorescence intensities consistent with the loss of VGLUT1 ${ }^{\text {VENUS }}$ vesicles from the synaptosomes. Contrary to this, the fluorescence intensity of isolated VGLUT1 ${ }^{\text {VENUS }}$ positive particles is preserved in our FSC-triggered experiments.

Finally, FM4-64 staining revealed a large pool of membrane containing particles in a smaller size range among which a population of VGLUT1 ${ }^{\text {VENUS }}$ positive particles is detected. FASS can enrich these fluorescently positive particles as analyzed by flow-cytometry.

\subsubsection{Evidence from Western blotting and immunofluorescence microscopy}

After FASS in FSC-mode, which analyzes particles in the size range of 0.75 to $1.5 \mu \mathrm{m}$ polystyrene beads, the sorted VGLUT1 ${ }^{\text {VENUS }}$ positive particles were not only enriched in VGLUT1 $^{\text {VENUS }}$ but also in VIAAT (inhibitory synaptosomes) and PLP (myelin) as analyzed by Western blotting. FASS of in FM-mode isolated VGLUT1 ${ }^{\text {VENUS }}$ particles in a smaller size range and also enriched VGLUT1 ${ }^{\text {VENUS }}$ as assessed by Western blotting. In stark contrast to the isolation in FSC-Mode, FASS in FM-mode, depleted in VIAAT (inhibitory synaptosomes), VAChT (cholinergic synaptosomes), PLP (myelin), GLT1 (astrocytic membranes), IBA1 (microglial particles) and VGLUT2 (other excitatory synaptosomes). Furthermore, Western blot analysis of VGLUT1 ${ }^{\text {VENUS }}$ positive particles isolated by FASS in FM-mode, showed that they also contain proteins corresponding to all functional elements expected in VGLUT1 synaptosome, including: (i) several SV-proteins and SV-associated proteins (Synaptophysin, Synapsin, SV2B) (ii) proteins involved in endocytosis of VGLUT1 SVs (EndophilinA1) (iii) Components of the release machinery (SNAP23/25/47 and ComplexinlI) (iv) mitochondrial proteins (VDAC1, TOM20) (v) components of the post-synaptic membrane (NL1, NR1, NR2A GluR2).

Through this set of Western blot experiments I therefore established that VGLUT1 $^{\text {VENUS }}$ synaptosomes isolated by FASS in FM-mode allowed the specific enrichment 
of VGLUT1 $1^{\text {VENUS }}$ synaptosomal proteins, while depleting proteins of other synaptosomes and glial contaminants.

\subsubsection{Evidence from electron and immunofluorescence microscopy}

Beyond biochemical identification of protein markers we wished to assess the integrity and purity of synaptosomes isolated by FASS through photonic and electron microscopic methods. In a recent publication, Fein and coworkers, sorted synaptosomal particles in the size range of .75-1.5 $\mu \mathrm{m}$ beads in FSC-mode and processed them for EM by negative staining with uranyl acetate (Fein et al., 2008). The images shown do not reveal the ultrastructure of the isolated particles, but merely demonstrate the presence of round membrane bounded particles of varying sizes (no further quantification of the data was provided). An accepted view of the size of small CNS synaptic elements is of 0.5 to $2 \mu \mathrm{m}$ as a whole with the presynapse being bigger than the post-synaptic specialization.

It may seem confounding that I detected synaptosomes in a size range below 0.75 $\mu \mathrm{m}$ in the calibration using light scattering of polystyrene beads in flow-cytometry. However, it is known that plastic beads and cells (synaptosomes) differ significantly in their refractive indices and therefore scatter light differently(Hoffman, 2005). Therefore, plastic beads only provide relative size references and can not easily be used to determine particle size exactly (Hoffman, 2005). Consequently the actual size of particles isolated by FASS may be larger than $0.75 \mu \mathrm{m}$. In agreement with this, my immunofluorescence and electron microscopy analyses of sorted VGLUT1 ${ }^{\text {VENUS }}$ synaptosomes demonstrated that most VGLUT1 $1^{\text {VENUS }}$ synaptosomes are detected within a size range of 0.5 to $2 \mu \mathrm{m}$. These results indicate that the particles we isolated are in a size range that is expected for synaptosomes and that the calibration with polystyrene beads underestimated the size of synaptosomes.

Furthermore, we could show that $75 \%$ of VGLUT1 ${ }^{\text {VENUS }}$ particles isolated through FASS can be stained with antibodies against the postsynaptic marker PSD-95. As expected, VGLUT1 $^{\text {VENUS }}$ and PSD-95 were not strictly colocalized, but rather facing each other. This indicated that at least $75 \%$ percent of VGLUT1 ${ }^{\text {VENUS }}$ positive particles have a postsynaptic density attached and further validates that the isolated particles correspond to intact synaptosomes. It would theoretically be possible that some VGLUT1 ${ }^{\text {VENUS }}$ positive particles have postsynaptic densities attached that contain little or no PSD-95. Therefore, the fraction of VGLUT1 ${ }^{\text {VENUS }}$ synaptosomes with a PSD attached might even be higher than $75 \%$.

Additionally electron microscopy of VGLUT1 ${ }^{\text {VENUS }}$ FASS samples showed that they contain intact synaptosomes, which can be identified by their size, vesicular and mitochondrial content presynaptically and the presence of a post-synaptic density. A quantification of systematically taken EM images revealed that FASS enriches in particles with a synaptic profile, while depleting non-synaptic particles. 
To summarize, the results obtained by microscopy convincingly demonstrate the enrichment in single, intact VGLUT1 ${ }^{\text {VENUS }}$ synaptosomes of a size range compatible with the average synaptic sizes in the CNS.

\subsubsection{Implications for the interpretation of earlier studies}

The present results demonstrate the presence of single, intact VGLUT1 ${ }^{\text {VENUS }}$ positive synaptosomes in FASS purified samples in a size range that is smaller than the size range analyzed or sorted in all previous flow-cytometric studies of synaptosomal preparations. In my hands the isolation of particles in the previously reported size range for synaptosomes (0.75-1.5 $\mu \mathrm{m}$ polystyrene beads) leads to the accumulation of aggregates. I cannot exclude that differences in instrumentation, sample preparation and the species studied account for the observed differences. However, the logical conclusion from my data is that many of the experiments reported by Gylys and coworkers and Wolf and colleagues analyzed and sorted aggregates (Fein et al., 2008; Gylys et al., 2000; Gylys et al., 2004a; Gylys et al., 2007; Gylys et al., 2004b; Wolf et al., 1991a). Therefore some of the results obtained in these studies are probably not correct and should be to re-evaluated using the methods implemented in the present study.

\subsection{Differential subcellular localization of several synaptic protein isoforms analyzed by FASS}

The characterization of sorted VGLUT1 ${ }^{\text {VENUS }}$ synaptosomes by western blotting, immunofluorescence and electron microscopy demonstrates the integrity and improved purity of the FASS preparation as compared to gradient purified synaptosomes. We therefore used FASS to study the association of several proteins with VGLUT1 ${ }^{\text {VENUS }}$ synaptosomes versus the total synaptosome preparation. For many synaptic proteins several closely related isoforms exist which together constitute a family of synaptic proteins. These functionally similar proteins often show differential expression patterns and differential subcellular localizations within the brain. If FASS of VGLUT1 ${ }^{\text {VENUS }}$ synaptosomes indeed allows the isolation of highly pure VGLUT1 $1^{\text {VENUS }}$ synaptosomes, the differential distribution of isoforms of synaptic proteins should be reflected in their differential enrichment/depletion in the FASS purified material. That this is indeed the case is illustrated by my Western blot analysis of sorted VGLUT1 $1^{\text {VENUS }}$ synaptosomes which showed a differential enrichment in the VGLUT1 ${ }^{\text {VENUS }}$ synaptosomes for closely related isoforms of SNARE proteins, SV2 proteins and of Complexins.

SNARE proteins mediate the fusion of synaptic vesicles with the plasma membrane during $\mathrm{Ca}^{2+}$-regulated exocytosis ((Söllner et al., 1993) for review see (Jahn and Scheller, 
2006)). More specifically, synaptic vesicle fusion is generally mediated by Synaptobrevin-2, Syntaxin-1 and SNAP-25. A close homolog of SNAP-25, SNAP-23 is also expressed in the brain. Immunofluorescence studies indicated a preferential association of SNAP25 excitatory VGLUT1 and of SNAP23 with excitatory VGLUT2 synapses respectively (Bragina et al., 2007). My data confirm the preferential association of SNAP25 with VGLUT1 synaptic terminals. On the other hand, SNAP23 was also enriched in the FASS sample, albeit to a lesser extent than SNAP25. The present results indicate that a large fraction of SNAP23 protein in the synaptosomal preparation is associated with the VGLUT1 ${ }^{\mathrm{VENUS}}$ synaptosomes. In support of this, it was recently reported that SNAP23 is a specific component of excitatory postsynaptic membranes where it affects the vesicular trafficking and surface expression of NMDA-receptors (Suh et al., 2010).

To ensure precise timing and regulation of neurotransmitter release, SNARE mediated SV exocytosis is regulated by Complexins (for review see (Brose, 2008)). Complexins may function by first promoting SNARE complex assembly and then "clamping" the activated SNARE complexes until $\mathrm{Ca}^{2+}$-influx triggers exocytosis through the calcium sensing protein Synaptotagmin (for review see (Südhof and Rothman, 2009)). Vertebrates express four isoforms of Complexins and it was reported that Complexinll was preferentially localized to excitatory synapses, while Complexinl was more specific to inhibitory synapses (Eastwood and Harrison, 2005; Harrison and Eastwood, 1998; Takahashi et al., 1995; Yamada et al., 1999). Using FASS, I found that within the synaptosomal preparation Complexinll was preferentially associated with VGLUT1 VENUS synaptosomes, while Complexinl was less enriched in sorted VGLUT1 ${ }^{\text {VENUS }}$ synaptosomes. This finding provided further evidence for a differential distribution of Complexin isoforms at subtypes of synapses in the forebrain. Also, it further validates the FASS-method, as our results are in agreement with the previous data on the distribution of Complexinl and II in the brain. It should be noted that functional analyses of Complexinl and Complexinll deficient hippocampal neurons found that they function redundantly at both GABAergic and glutamatergic synapses (Reim et al., 2001). For this reason the relevance of the differential distribution of Complexin isoforms for synaptic physiology and pathology remains to be elucidated.

The proteins of the SV2 protein family is composed of three closely related proteins, $\mathrm{SV} 2 \mathrm{~A}, \mathrm{~B}$ and $\mathrm{C}$. These synaptic vesicle proteins have 12 transmembrane domains and are heavily glycosylated. The exact mechanism of SV2 action is not completely clear, but their importance in the regulation of synaptic transmission is highlighted by the fact that mice lacking SV2A or SV2A and SV2B die within 3 weeks postnatally due to heavy epileptic seizures (Crowder et al., 1999; Janz et al., 1999). SV2A and SV2B display differential expression patterns (Bajjalieh et al., 1994). While SV2A is more ubiquitously distributed, SV2B displays a more restricted excitatory expression pattern. These differences were 
recently supported in a study comparing immunoisolated VGLUT1 and VIAAT containing SVs (Grønborg et al., 2010). Using FASS, I found that SV2B is specifically co-enriched in VGLUT1 $1^{\text {VENUS }}$ synaptosomes, while SV2A is partially depleted from the VGLUT1 $1^{\text {VENUS }}$ synaptosomes. My observations are therefore consistent with the previously observed distributions of SV2A and SV2B.

FASS allowed detecting the differential subcellular localization of three pairs of closely related isoforms of synaptic proteins. The results obtained for the differential distribution of SNAP23 and SNAP25, Complexinl and Complexinll as well as SV2 and SV2B by analysis of FASS samples is in agreement with previous findings. These experiments therefore provide a strong validation of the strength of the FASS method to further purify VGLUT1 ${ }^{\text {VENUS }}$ synaptosomes from the conventional preparation. Importantly, these findings also mean that FASS can be used to characterize the differential subcellular localization of protein isoforms of previously unknown distribution.

\subsubsection{FASS provides evidence that Neuroligin3 and 4 are not present at VGLUT1 synapses}

In the rodent brain four Neuroligin isoforms (Neuroligin1-4) are expressed. Neuroligins are postsynaptic cell adhesion molecules that are important for proper synapse maturation and function (for review see (Südhof, 2008)). Using FASS, I have found that Neuroligin1 was co-enriched with VGLUT1 VENUS synaptosomes, while Neuroligin2 was strongly depleted from this sample. These findings are in accordance with several earlier studies that established the specificity of NL1 for excitatory synapses and NL2 for inhibitory synapses (for review (Lisé and El-Husseini, 2006; Südhof, 2008)). The differential enrichment in Neuroligin1 and the depletion of Neurologin2 in FASS samples once more validates the purity of the sorted VGLUT1 ${ }^{\text {VENUS }}$ synaptosomes and means that FASS can be used to test for the specific association of a given postsynaptic molecule with the PSD of VGLUT1 ${ }^{\text {VENUS }}$ synapses

So far the cellular and subcellular localization of Neuroligin3 and Neuroligin4 have not been characterized. Indeed, Neuroligins are involved in complex protein interactions at PSDs and are difficult to stain with antibodies in situ. Nevertheless, antibodies directed against Neuroligins perform well in Western blotting. Using FASS, I discovered that Neuroligin3 and Neuroligin4 are depleted from VGLUT1 ${ }^{\text {VENUS }}$ synaptosomes. Neuroligin3 and Neuroligin4 only showed a slight tendency to be less strongly depleted than Neuroligin2. These findings demonstrate that NL3 and NL4 are not associated with the majority of VGLUT1 ${ }^{\text {VENUS }}$ synapses, thus providing a first indication that these Neuroligin isoforms are present at other subtypes of synapses in the forebrain. These results are supported by preliminary immunofluorescence data from our laboratory. 


\subsubsection{NR2A but not NR2B, is enriched at VGLUT1 synapses}

The effect of a neurotransmitter depends on the function of its cognate receptor proteins in the postsynaptic membrane. The types and numbers of different neurotransmitter receptors at synapses is therefore a defining feature in the functional specialization of a given synapse.

In adult neurons, neither NR2A or NR2B can be considered exclusively synaptic or extrasynaptic, but pharmacological and electrophysiological data indicate that NR2B is preferentially localized to extrasynaptic sites, while NR2A is predominantly localized synaptically (Ivanov et al., 2006; Rosenmund et al., 1995; Thomas et al., 2006; Tovar and Westbrook, 1999) for review see (van Zundert et al., 2004). A preferential localization for NR2B to extrasynaptic sites was also found in immunocytochemical studies on dissociated cultures reporting up to 83-90 \% of NR2B at extrasynaptic sites (Groc et al., 2009; Groc et al., 2004; Groc et al., 2006). Nevertheless, at the level of PSD purifications both NR2A and NR2B are found in similar levels and at similar enrichment factors, and also several immunohistochemical studies did not find a preferential distribution of NR2B to synaptic or extrasynaptic sites (Al-Hallaq et al., 2001; Harris and Pettit, 2007; Lee et al., 2001a; Petralia et al., 2010). Interestingly, one study showed that there is a substantial overlap of extrasynaptic NMDA receptor staining with labeling for PSD95/93 and SAP102, indicating that these receptors can also be tightly anchored by scaffolding proteins. The extrasynaptic accumulations of NMDA receptors on dendrites usually corresponded to points of close contacts with other cells. These contacts included axon/dendrite contacts and glia/dendrite contacts. (Petralia et al., 2010).

In my Western blot analysis of FASS purified VGLUT1 ${ }^{\text {VENUS }}$ synaptosomes I found that within the synaptosomal preparation, NR2A is significantly more specific to VGLUT1 synapses than NR2B. These results indicate that within in the synaptosomal preparation a large fraction of NR2B protein is present in other structures than VGLUT1 synaptosomes, supporting the view that NR2B is preferentially localized to extrasynaptic sites. These data represent the first biochemical evidence for a difference in synaptic vs. extrasynaptic localization of the NR2A and NR2B subunits. Further experiments will be needed to characterize the distribution of NR2A and NR2B within the FASS purified synaptosomal preparation. In any case, the present data imply that the conventional, detergent based PSD purifications are not entirely selective for postsynapses and also include receptors clustered at extrasynaptic sites. FASS may therefore be useful in separating synaptic and extrasynaptic pools of receptors for biochemical analyses in physiology and disease. 


\subsection{FASS allows the analysis of a VGLUT1-specific synaptic proteome}

Proteomics has been applied extensively in neuroscience in order to study the protein composition of the brain. However, the analysis of whole tissue samples identifies mainly the most abundant proteins important for general brain functions. As outlined in the introduction and covered in many recent reviews on organelle and neuroproteomics, reducing the level of complexity of the sample under study is crucial for the identification of proteins that are specific to a given organelle but of low abundance (Tribl et al., 2006; Tribl et al., 2008). Therefore, methods of subcellular fractionation have been essential for identifying the specific composition of several substructures within different brain regions, such as synaptosomes, synaptic membranes and also synaptic vesicles ((Tribl et al., 2006; Tribl et al., 2008) and see Introduction 1.6).

As mass-spectrometric identification of proteins becomes increasingly sensitive, an ever-increasing number of proteins can be identified. A downside of this increased sensitivity is that it becomes difficult to separate interesting low abundance proteins from unspecific proteins originating from contaminations copurified in the given subcellular fraction. The quantitative, comparative analysis of two fractions is an excellent approach to allow the differentiation of specific and non-specific components.

\subsubsection{Quantitative analysis by spectral counting}

Analyzing the protein complement of gradient purified synaptosomes and of VGLUT1 $1^{\text {VENUS }}$ synaptosomes isolated by FASS, allowed for the first time a proteomic comparison of a subset of intact synaptosomes with the whole synaptosomal population of the forebrain. In total, I identified over 2000 proteins in the sorted VGLUT1 ${ }^{\text {VENUS }}$ synaptosomes. Using label-free quantification through spectral counting with stringent criteria on the quality of protein and peptide identification I quantified 1075 proteins, 434 of which showed a preference for the sorted VGLUT1 ${ }^{\text {VENUS }}$ synaptosomes and 163 of which were enriched in the VGLUT1 $1^{\text {VENUS }}$ synaptosomes by a factor of 2 or more. I thus identified a number of proteins that are specifically enriched in VGLUT1 synapses and separated them from proteins originating from glial contaminations, non-synaptic neuronal particles or proteins specific to other synaptic subtypes.

Spectral counting is regarded a semi-quantitative approach in mass spectrometric analyses. For 19 proteins quantified by spectral counting, I also obtained a quantification of their enrichment or depletion in sorted VGLUT1 ${ }^{\text {VENUS }}$ synaptosomes by Western blotting. The trends of enrichment or depletion were correctly reflected by the spectral counts for 18 of the 
tested 19 proteins. Thus spectral counting provided reliable information regarding the enrichment or depletion of proteins in VGLUT1 ${ }^{\text {VENUS }}$ synaptosomes after FASS.

Among the 163 proteins that were enriched in sorted VGLUT1 $1^{\text {VENUS }}$ by a factor of 2 or more were many well-known components of all functional parts of the synapse. These included:

(i) Signaling proteins such as CamKII $\alpha, \beta, \gamma, \delta$ and $\beta$-Catenin

(ii) Trafficking proteins such as Syntaxin-6, RABs, and ARF proteins

(iii) Proteins necessary for neurotransmitter loading including several subunits of the vesicular $\mathrm{H}^{+}$-ATPase and VGLUT1

(iv) Other SV proteins like Synaptophysin, SV2A, SV2B and SV31

(v) Active zone proteins such as Munc13-1, CASK and Liprin- $\alpha$

(vi) Proteins important in synaptic vesicle exocytosis such as Synaptobrevin-2 (VAMP2), Syntaxin-1, SNAP25, Synaptotagmin I and XII, Munc18-1, CAPS1 and Tomosyn

(vii) Proteins involved in endocytosis of SVs, such as NSF, EndophilinA1, Clathrin heavy chain, AP2 and AP3

(viii) Mitochondrial proteins such as $\mathrm{NADH}$ dehydrogenase and subunits of the $\mathrm{F}_{1} \mathrm{~F}_{0}$-complex

(ix) Proteins of the PSD such as PSD95, PSD93

Furthermore 343 proteins were depleted in the VGLUT1 ${ }^{\text {VENUS }}$ isolated by FASS by a factor of two or more according to the quantification by spectral counting. These included myelin proteins such as PLP and MBP, but also proteins known to be specific for inhibitory synapses such as $\mathrm{GABA}_{\mathrm{A}}$-receptor, Calretinin and Neuroligin2.

Together, these data provide a very strong validation of the purity of the VGLUT1 $1^{\text {VENUS }}$ synaptosomes isolated by FASS at a larger scale. Importantly these data also show that the enrichment of a protein in sorted VGLUT1 ${ }^{\text {VENUS }}$ synaptosomes as assessed by spectral counting is a reliable indicator for this protein to localize to VGLUT1 synapses. Therefore, many of the proteins that were enriched in sorted VGLUT1 ${ }^{\text {VENUS }}$ synaptosomes and that were previously not shown to localize to VGLUT1 synapses are probably also present at VGLUT1 synapses. To test this hypothesis we selected several proteins from the list of 163 enriched proteins and investigated their potential localization.

\subsubsection{Transcriptome comparison as a filter for candidate selection}

As mentioned above, the quantitative comparison of VGLUT1 ${ }^{\text {VENUS }}$ synaptosomes with gradient purified synaptosomes allowed the identification of 163 proteins, which were significantly enriched in VGLUT1 ${ }^{\text {VENUS }}$ synaptosomes. To further validate the specificity of my 
FASS protocol and analysis I complemented the proteomic data with cell-type specific gene expression data (Cahoy et al., 2008). The corresponding analysis demonstrated that proteins enriched in VGLUT1 ${ }^{\text {VENUS }}$ synaptosomes after FASS were largely neuron specific, while many of the proteins depleted from this the FASS sample showed a glia specific gene expression. Importantly several genes which were depleted from VGLUT1 ${ }^{\text {VENUS }}$ synaptosomes, but show a neuron specific gene expression, such as Calretinin and $G A B A_{A^{-}}$ receptor, are known to be specific components of GABAergic neurons and synapses.

Taken together the results of this transcriptome comparison corroborate the specificity of my analysis of FASS purified VGLUT1 ${ }^{\text {VENUS }}$ synaptosomes by spectral counting. Furthermore, I used the analysis of transcriptome data to help guide my selection of protein candidates. To this end I first selected only those of the 163 enriched proteins that also showed a differential and high neuronal gene expression (see Nmax, NmaxO, NmaxA in Figure 25). From this subset I selected four protein candidates based on literature analysis and the availability of antibodies. These proteins were FXYD6, Ly6H, Cavin1 and Cavin2. A fifth candidate protein I choose is TPD52. TPD52 was enriched 3.33-fold in sorted VGLUT1 ${ }^{\text {VENUS }}$ synaptosomes as assessed by spectral counting, but in the transcriptome data it also showed high gene expression in glial cell types. However, MAL2, a protein known to interact with TPD52, had recently been identified as a specific component of VGLUT1 containing synaptic vesicles (Grønborg et al., 2010). Using a variety of methods I now provide first evidence that three of the five selected candidates are probably localized to or associated with VGLUT1 synapses.

\section{$4.5 \quad F X Y D 6$}

The excitability of neurons, synaptic transmission and plasma membrane transport of neurotransmitters all depend on the maintenance of $\mathrm{Na}^{+}, \mathrm{K}^{+}, \mathrm{Cl}^{-}$or $\mathrm{Ca}^{2+}$ gradients across the neuronal plasma membrane. (R. Kandel et al., 2000). The gradients of $\mathrm{Na}^{+}$and $\mathrm{K}^{+}$across the cell membrane are maintained by the $\mathrm{Na}^{+} / \mathrm{K}^{+}$-ATPase (SKOU, 1957). Apart from restoring the $\mathrm{Na}^{+}$and $\mathrm{K}^{+}$gradients during and after an action potential the $\mathrm{Na}^{+} / \mathrm{K}^{+}$-ATPase also provides the electrochemical driving force for the maintenance of $\mathrm{Cl}^{-}$and $\mathrm{Ca}^{2+}$ gradients by secondary active transport mechanisms (R. Kandel et al., 2000).

The function of the $\mathrm{Na}^{+} / \mathrm{K}^{+}$-ATPase can be regulated by small membrane proteins of the FXYD protein family (for reviews see (Garty and Karlish, 2006; Geering, 2006)). Mammals express seven different isoforms of FXYD proteins (FXYD1-7), which can bind to and affect the $\mathrm{Na}^{+} / \mathrm{K}^{+}$-ATPase by changing its apparent affinity for $\mathrm{Na}^{+}, \mathrm{K}^{+}$or ATP and its catalytic activity. The $\mathrm{Na}^{+} / \mathrm{K}^{+}$-ATPase is composed of one of four alpha $(\alpha 1-4)$ and one of four beta subunits( $\beta 1-4)$. Different $\mathrm{Na}^{+} / \mathrm{K}^{+}$-ATPase isozymes are differentially affected by FXYD 
proteins, and thus the properties of $\mathrm{Na}^{+} / \mathrm{K}^{+}$-exchange depend on the combination of $\mathrm{Na}^{+} / \mathrm{K}^{+}$ATPase isozyme and FXYD protein isoform expressed in a given cell (Garty and Karlish, 2006; Geering, 2006). Due to their differential tissue-specific expression patterns, FXYD proteins have been proposed to fine-tune the function of the $\mathrm{Na}^{+} / \mathrm{K}^{+}$-ATPase to the specific needs of a given tissue.

In the brain FXYD6 and FXYD7 are the predominant isoforms. FXYD7 has been found in neurons and glia. In neurons it can colocalize with synaptophysin and it specifically binds to and affects $\alpha 1 \beta 1$-containing isozymes of the $\mathrm{Na}^{+} / \mathrm{K}^{+}$-ATPase isozymes (Béguin et al., 2002). Functionally, FXYD7 decreases the apparent affinity of the $\mathrm{Na}^{+} / \mathrm{K}^{+}$-ATPase for $\mathrm{K}^{+}$ and thus allows maximal $\mathrm{K}^{+}$-transport at high $\mathrm{K}^{+}$concentrations, e.g. during periods of high frequency stimulation.

FXYD6 expression is highly specific to the brain and FXYD6 can be localized to the membrane of neuronal somata and dendrites in cortex, hippocampus and cerebellum (Kadowaki et al., 2004; Yamaguchi et al., 2001). In addition, FXYD6 is expressed in the inner ear, where its expression levels during development correlate with establishment of the ion-homeostasis necessary for proper auditory signaling (Delprat et al., 2007a; Delprat et al., 2007b). FXYD6 decreases the apparent $\mathrm{K}_{1 / 2}\left(\mathrm{Na}^{+}\right)$of $\alpha 1 \beta 1 \mathrm{Na}^{+} / \mathrm{K}^{+}-\mathrm{ATP}$ ases and increases the $\mathrm{K}_{1 / 2}\left(\mathrm{Na}^{+}\right)$of $\alpha 1 \beta 2$.-isozymes. Thus FXYD6 allows the $\alpha 1 \beta 1$ isozyme to respond to increases in intracellular $\mathrm{Na}^{+}$concentrations with increased $\mathrm{Na}^{+} / \mathrm{K}^{+}$-exchange, even at high intracellular $\mathrm{Na}^{+}$concentrations. On the other hand regulation of the $\alpha 1 \beta 2$ isozyme by FXYD6 allows for maximal extrusion of $\mathrm{Na}^{+}$-ions even at low sodium concentrations (Delprat et al., 2007b). Interestingly, the $\alpha 1 \beta 1$-isozyme was found in dendrites of auditory neurons together with FXYD6. FXYD6 might therefore be important to maintain the excitability of these neurons even during phases of high synaptic activity (Delprat et al., 2007b).

The FASS purified VGLUT1 ${ }^{\text {VENUS }}$ synaptosomes contained both FXYD6 and FXYD7. While FXYD7 showed only moderate enrichment in VGLUT1 ${ }^{\text {VENUS }}$ synaptosomes according to spectral counting (enrichment 1.1-fold), FXYD6 was substantially enriched in VGLUT1 ${ }^{\text {VENUS }}$ synaptosomes by a factor of 2 (see Table 2). In fact, FXYD6 was hardly detected in the unsorted sample (normalized spectral count of 2.32, based on $1 \mathrm{HQ}$ peptide) but robustly detected in the sorted sample (normalized spectral count 4.39 , based on $2 \mathrm{HQ}$ peptides). In addition, the transcriptome data of Cahoy et al. (2008) indicate a preferentially neuronal expression of FXYD6. In light of these observations, I selected FXYD6 for further analysis regarding its localization to VGLUT1 synapses.

\subsubsection{FXYD6 at VGLUT1 synapses}

Complementing the previous findings on the localization of FXYD6 in neuronal cell bodies and dendrites, my proteomic analysis of VGLUT1 ${ }^{\text {VENUS }}$ synaptosomes isolated by 
FASS indicated that FXYD6 is present at VGLUT1 synapses. Analysis by Western blotting confirmed the enrichment of FXYD6 in the sorted VGLUT1 ${ }^{\text {VENUS }}$ synaptosomes, indicating that even quantifications based on relatively low spectral counts may correctly reflect the relative distribution of a protein between two samples (Figure 27A). Consistent with the enrichment in VGLUT1 ${ }^{\text {VENUS }}$ synaptosomes and with its potential effect on the $\mathrm{Na}^{+} / \mathrm{K}^{+}$ATPase at the neuronal plasma membrane FXYD6 was found to be enriched in synaptic plasma membrane fractions (LP1B) upon subcellular fractionation of forebrain tissue (Figure 27B). Furthermore, Western blot analyses of brain region specific tissue homogenates revealed that FXYD6 is expressed throughout the brain. This result is consistent with our observations in immunostainings of brain sections as well as the Western blot analyses performed in earlier studies (Kadowaki et al., 2004).

Using immunofluorescence staining of cultured neurons and brain sections, we confirmed earlier observations that FXYD6 is present on the plasma membrane of neuronal cell bodies and dendrites (Figure 28 and Figure 29). FXYD6 does not seem to be expressed in GFAP positive astrocytes cocultured with the neurons from the hippocampus (Figure 28B).

Using Immuno-EM we localized FXYD6 at the plasma membrane of axons, axon terminals, and to a lesser extend, also of synaptic vesicles (Figure 29). Importantly, we found FXYD6 to be colocalized with presynaptic VGLUT1 in cultured hippocampal neurons and in the hippocampus and cortex in situ. Father analyses revealed that FXYD6 is also present at VGLUT2 expressing synapses in cultured hippocampal neurons and that in the hippocampus it is not present at axonal varicosities of inhibitory neurons (Figure 29). Together these results provide evidence for the presence of FXYD6 at VGLUT1 synapses, which I originally inferred from our analysis of FASS purified VGLUT1 ${ }^{\text {VENUS }}$ synaptosomes. Additionally we could observe that while FXYD6 is strongly expressed in the VGLUT1 expressing granule cells of the cerebellum, the inhibitory Purkinje cells do not express FXYD6 (data not shown). These results, together with the segregation of GAD65/67 and FXYD6 stainings in the hippocampus, indicate a preferential localization of FXYD6 in excitatory neurons.

My results indicate that FXYD6 is localized to VGLUT1 synapses and is expressed preferentially in excitatory neurons. This substantiates the usefulness of my FASS protocol for the identification of novel components of VGLUT1 synapses.

Furthermore, the fact that FXYD6 was localized in presynaptic terminals and dendrites of neurons raises the possibility that FXYD6 functions in regulating the transport properties of $\mathrm{Na}^{+} / \mathrm{K}^{+}$-ATPases in subcellular compartments involved in excitatory neurotransmission. Future studies will have to address the potential role of FXYD6 on presynaptic and post-synaptic aspects of synaptic transmission.

Given that FXYD6 has a differential effect on the properties of different $\mathrm{Na}^{+} / \mathrm{K}^{+}$ATPase isozymes it will be interesting to correlate their respective cellular and subcellular 
localization. Furthermore, phosphorylation of FXYD isoforms can alter their effect on the $\mathrm{Na}^{+} / \mathrm{K}^{+}$-ATPase (Arystarkhova et al., 2002). In synaptic transmission, local signaling cascades might therefore allow for regional adaptations of the transport properties of the $\mathrm{Na}^{+} / \mathrm{K}^{+}$-ATPase through modification of FXYD proteins. Finally it is known that $\mathrm{Na}^{+} / \mathrm{K}^{+}$ATPase isozymes also display differential expression patterns within the brain(Moseley et al., 2003; Richards et al., 2007). Together, the differential distribution of FXYD proteins and $\mathrm{Na}^{+} / \mathrm{K}^{+}$-ATPase isozymes may allow for complex fine-tuning of the ion-homeostasis of neurons.

\subsection{TPD52}

The tumor protein D52 (also named CRHSP-28,CSPP28 or R10) was initially identified because of its overexpression in several human cancers and cancer cell lines (Byrne et al., 1996). Since then multiple lines of evidence implicate TPD52 in secretory processes:

(i) TPD52 functions in $\mathrm{Ca}^{2+}$-regulated exocytosis in secretory cells in the digestive system, namely the gastric chief cells and pancreatic acinar cells (Groblewski et al., 1996; Parente et al., 1996; Thomas et al., 2001). (ii) TPD52 is highly expressed in mature B-cells and plasma cells where it has been implicated in the regulation secretory processes involving the $\mathrm{Ca}^{2+}$-dependent binding of TPD52 to AnnexinVI (Tiacci et al., 2005). (iii) A recent study implicated TPD52 in the regulation of the trafficking of lysosomal proteins to the plasma membrane (Thomas et al., 2010). (iv) TPD53 (D53) a close homolog of TPD52 can bind to neuronal SNARE proteins in vitro and can be immunoprecipitated with synaptobrevin-2 from cell extracts (Proux-Gillardeaux et al., 2003) (v) All members of the TPD52 protein family can interact with MAL2 in yeast-two hybrid interaction assays, and MAL2 has recently been identified as a specific component of VGLUT1 containing synaptic vesicles (Grønborg et al., 2010).

TPD52 mRNA and protein are found in the brain and immunofluorescence data indicate that TPD52 is present in as yet unidentified granule rich cells in the hippocampus (Chew et al., 2008; Groblewski et al., 1999; Parente et al., 1996). Additional evidence for the expression of TPD52 was presented by functional correlation of microarray based gene expression data, which identified TPD52 as a gene, whose expression is highly correlated with that of genes that encode neuronal and synaptic proteins (Oldham et al., 2008). In my proteomic analysis of FASS samples, TPD52 was enriched 3.33-fold in sorted VGLUT1 ${ }^{\text {VENUS }}$ synaptosomes as assessed by spectral counting. Taking into account all the evidence mentioned above, it is a plausible hypothesis that TPD52 can localize to and function at VGLUT1 synapses. 


\subsubsection{TPD52 at VGLUT1 synapses}

Although multiple lines of evidence have implicated TPD52 in vesicle trafficking and secretion its cellular and subcellular expression in the brain have not been studied so far. Based on the quantitative comparative proteomic analysis of unsorted and sorted VGLUT1 ${ }^{\text {VENUS }}$ synaptosomes, I inferred that TPD52 localizes to VGLUT1 containing synapses in the forebrain. Using immunofluorescence staining, I found that TPD52 is present in most VGLUT1 $1^{\text {VENUS }}$ synaptosomes isolated by FASS (Figure 30D). Western blotting and immunofluorescence data showed that TPD52 is expressed in different brain regions, with highest levels in olfactory bulb and brainstem. Consistent with a synaptic localization, I found that TPD52 was strongly enriched in the fraction of soluble synaptic proteins (LS2) upon subcellular fractionation of forebrain tissue. Immunostaining of TPD52 In cultured hippocampal neurons revealed a punctate, somatodendritic and synaptic staining pattern. TPD52 was partially colocalized with VGLUT1 at presynapses and with PSD95 at postsynapses (Figure 30). Furthermore, analysis of double immunofluorescence stainings with the inhibitory marker GAD65 showed that TPD52 almost absent from inhibitory synapses of cultured hippocampal neurons (Figure 30C). Together these results provide firm evidence for the synaptic localization of TPD52, thus confirming the original inference based on proteomic analysis of VGLUT1 ${ }^{\text {VENUS }}$ synaptosomes isolated by FASS.

In view of the present data and considering the fact that TPD52 has been implicated in calcium dependent vesicle trafficking and binds to the SV protein MAL2, it is likely that TPD52 is involved in SV trafficking or other membrane trafficking steps in the pre- and postsynapse. This could be tested by analyzing protein-protein interactions of TPD52, or by knock-down of TPD52 together with live-imaging of synaptic vesicle trafficking and electrophysiological measurements in cultured hippocampal neurons

\section{$4.7 \quad$ Ly6H}

Outside the brain, Ly6-family proteins have been implicated in different cellular processes in a variety of tissues, including, T-cell activation, protection from complement system components, regulation of cell-adhesion at paranode-like septate junctions and regulation of nicotinic acetylcholine receptors (for review see (Holford et al., 2009)). Within the brain, members of the Ly6 family, such as Lynx1, Lynx2 and Lypd6 affect the function of nicotinic acetylcholine receptors (Darvas et al., 2009; Miwa et al., 1999; Miwa et al., 2006; Tekinay et al., 2009). Ly6H on the other had no effect on nicotinic acetylcholine receptors in the same experiments (Tekinay et al., 2009). Ly6H mRNA expression levels were highest in cerebral cortex, amygdala, hippocampus and subthalamic nucleus, while no Ly6H 
expression was detected in cerebellum, spinal cord, corpus callosum and thalamus (Apostolopoulos et al., 1999; Horie et al., 1998). Using in-situ hybridization, (Dessaud et al., 2006) showed Ly6H mRNA expression in the ventral neural tube of developing embryos and also in the adult hippocampus and cortex.

My analysis proteomic analysis of sorted VGLUT1 $1^{\text {VENUS }}$ synaptosomes isolated by FASS indicated that Ly6H was enriched in VGLUT1 ${ }^{\text {VENUS }}$ synaptosomes. In addition, transcriptome data of Cahoy et al. (2008) indicated that Ly6H is specifically expressed in neurons. Therefore, I investigated the localization of Ly6H to VGLUT1 synapses by various techniques.

The Ly6H antibody that was available in this study recognized several bands of different apparent molecular weight in Western blots of brain tissue homogenates. According to previous studies $\mathrm{Ly} 6 \mathrm{H}$ is not expressed in cerebellum. In agreement with these findings a protein recognized at an apparent molecular weight of $20 \mathrm{kDa}$ was detected in Ly6H Western blots of different brain region tissue homogenates, but not in cerebellum. Interestingly this protein was strongly enriched in synaptic plasma membranes upon subcellular fraction of forebrain tissue. Furthermore, the Ly6H antibody stained several synapse rich regions of the brain in situ but not the cerebellum. Notably, the immunofluorescence staining of Ly6H in brain sections also detects very strong signals in the corpus callosum and in the meninges. Due to the lack of specificity of the antibody in Western blotting of brain tissue homogenates, Ly6H immunoreactivity in brain sections may not be specific as well. Therefore one cannot draw reliable conclusions from these stainings.

In contrast to brain tissue homogenates, lysates of cultured primary hippocampal neurons contained only one main protein band that was detected by the Ly6 $\mathrm{H}$ antibody and not present in cultured cerebellar neurons. Therefore, the immunofluorescence signal for Ly6H in cultured primary hippocampal neurons may be more specific than the stainings in brain sections. In cultured primary hippocampal neurons Ly6H staining was occasionally observed in close apposition to VGLUT1 synapses. Additionally, $60 \%$ of sorted VGLUT1 $^{\text {VENUS }}$ positive synaptosomes were also positive for Ly6H. Together, with the enrichment of Ly6H protein in synaptic plasma membrane fractions these results hint at a possible localization of Ly6H to VGLUT1 synapses. However, the results obtained so far are confounded by the lack of specificity of the Ly6H antibody and more specific antibodies for Ly6H will be necessary to validate the localization of Ly6H to VGLUT1 synapses.

\subsection{Potential improvements and future applications of FASS}

In the present study, I show that subpopulations of synaptosomes can be isolated for subsequent biochemical analysis using FASS when VGLUT1 ${ }^{\text {VENUS }}$ mice are used as a 
source of fluorescent synaptosomes. This novel protocol provides an important methodological advance with many possible applications in neuroscience research. In addition this new methodological approach allowed me to address several biological questions and to identify new synaptic proteins. Further improvements of various aspects of the method as well as expected technological advancements will further enhance the usefulness of the FASS methodology and its applications.

\subsubsection{Potential for improvements of the current FASS-method}

Freshly prepared synaptosomes can be considered as live particles, whose quality deteriorates with longer durations of experiments. The subcellular fractionation prior to FASS requires roughly $3 \mathrm{~h}$ in the current protocol, and from one brain one can obtain mg quantities of synaptosomal protein. However, the following sorting of VGLUT1 ${ }^{\text {VENUS }}$ synaptosomes only yields roughly $0.8-1 \mu \mathrm{g}$ of synaptosomal protein with sort times of 6-8 $\mathrm{h}$. I observed that during longer sorts the sample quality decreased. Thus, throughput of the cell-sorter is a major factor that limits the amounts of sorted synaptosomes that can be isolated during a single FASS experiment.

Since in the flow-cytometric analysis the non-fluorescent and the fluorescent particles are not completely separated, improvements in the sensitivity of the fluorescence detection system could increase the fraction of VGLUT1 ${ }^{\text {VENUS }}$ positive synaptosomes available for FASS. The maximum analysis rate of the instrument during sorting is relatively fixed. Therefore, increasing the fraction of positive particles will increase the frequency by which particles can be sorted and will thus reduce the total time of the procedure. Potential technical modifications that would allow for a more sensitive fluorescence detection would be the optimization of fluorescence filter sets, an excitation optimized for VENUS at $514 \mathrm{~nm}$ and as well as the selection of the optimal PMT (photo-multiplier-tube) used for detection of different wavelengths.

Furthermore, recent experiments performed by Etienne Herzog on a different sort platform in Paris suggest that the establishment of custom procedures for laser alignment and drop-delay during instrument set-up may contribute to increase the yield during microparticle analysis and sorting.

An additional way to improve the protein yield of protein per experiment would be to optimize the sample recovery after FASS. Filtration on $0.22 \mu \mathrm{m}$ polycarbonate filters recovers $56 \%$ of the initial protein. By using different methods, such as adsorption to a column carrying a high affinity antibody or a lectin with affinity to synaptosomes or alternative methods of protein precipitation could be explored in order to achieve higher recovery rates.

The preparation of synaptosomes using sucrose as a gradient material has been shown to yield optimal results regarding the purify of the synaptosomal preparation 
(Whittaker, 1993). However, the use of non-viscous gradient materials such as Percoll $®$ might significantly reduce the time required for sample preparation and would thus reduce the overall time required for the procedure (Dunkley et al., 1986).

Another point for improvement of FASS would be to increase the purity of the sorted VGLUT1 ${ }^{\text {VENUS }}$ synaptosomes to a level of more than $99 \%$, as is commonly achieved in the isolation of cells using FACS. The two limiting factors in this respect are the sensitivity and resolution of the light-scattering detection systems on the FACSAria and the sensitivity of fluorescence detection. Improvement of the resolution and sensitivity of the light-scattering detection would allow for proper doublet-discrimination using pulse-geometry by plotting FSC-H vs. FSC-A and SSC-H vs. SSC-A and could thus help to avoid the sorting of coinciding particles. Currently the FACSAria does not have any resolution in the size range of particles that I was isolating. Therefore attempts of introducing doublet discrimination into our sort strategy did not have any effect on sort purity (data not shown). A different sort platform that has recently been developed implements detection of microparticles with more sophisticated light-scatter detection systems and has been reported to resolve particles as small as $0.1 \mu \mathrm{m}$ (http://www.bdbiosciences.com/instruments/influx/index.jsp, 29-09-2010). This would be very helpful for the optimization of the FASS protocol.

\subsubsection{Potential applications of FASS}

In the present study, I developed a method to purify VGLUT1 ${ }^{\text {VENUS }}$ synaptosomes by FASS, which yields synaptosomes of a purity that substantially exceeds the purity of conventionally prepared synaptosomes. Furthermore, the selective isolation of VGLUT1 ${ }^{\text {VENUS }}$ containing particles allowed for the specific enrichment of VGLUT1 synapses over other types of synapses and extrasynaptic neuronal contaminations present in starting synaptosomal preparation. I also demonstrated that FASS samples can be analyzed by Western blotting and proteomic techniques to investigate differential protein expression between samples.

As outlined in the introduction, major brain disorders such as Alzheimer's disease or Huntington's disease affect protein expression or localization at excitatory synapses. Therefore, FASS of VGLUT1 $1^{\text {VENUS }}$ synaptosomes might be a valuable tool in the investigation of VGLUT1-synapse specific alterations in protein expression or localization in the context of mouse models of these brain disorders.

Furthermore, specific presynaptic marker proteins for other neurotransmitter systems could be labeled in a similar way as VGLUT1 $1^{\text {VENUS }}$ and thus allow the isolation of synaptosomes of a variety of neurotransmitter-phenotypes using FASS. Comparative 
proteomic analyses of different synaptosome subpopulations isolated by FASS would allow to screen for neurotransmitter-system specific differences in synaptic protein composition.

One can envision that in the future FASS samples can also be used for functional assays. Since VGLUT1 $1^{\text {VENUS }}$ synaptosomes isolated by FASS are largely devoid of contaminations by glial membranes, FASS may eventually allow to address unresolved scientific questions such as the plasma membrane glutamate uptake at presynapses. Direct uptake of glutamate by presynapses has been suggested since many years (for review see (Danbolt, 2001)), but the studies on the presynaptic uptake of glutamate have always been blurred by the massive expression of GLT1 and GLAST on astrocytic processes. However significant improvements of sample yield will be necessary to allow such experiments.

In a longer-term perspective, one of the great potentials of FASS lies in the ability of flow cytometry to perform multiparametric phenotyping of each analyzed particle separately. The separation of synapses into neurotransmitter phenotypes may therefore only represent the beginning. In the future, a combination of spectrally separable fluorescently labeled genetic markers, with surface labeling of subtype specific proteins such as neurotransmitterreceptor subunits may allow for the definition and isolation of very specific synaptosome subpopulations using FASS. 


\section{$5 \quad$ Appendix}

\section{Appendix 1: Excursion into the presentation of flow-cytometry data}

AREA, WIDTH AND HEIGHT:

When a particle is analyzed by flow cytometry it passes through the laser beam and is continuously measured. Therefore the signal for each individual particle can be described by three basic parameters: The height $(H)$ measures the maximum intensity of the signal. The width $(\mathrm{W})$ measures the time over which the signal was recorded and the area $(A)$ measures the integrated signal over the time it was recorded (see below).

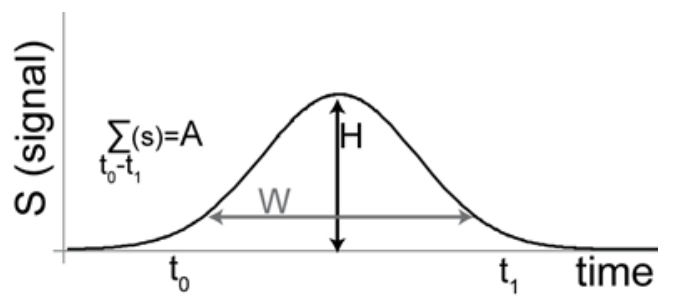

FSC:

FSC refers to "forward scatter" and measures scattering of laser light at low (forward) angles. Even though this is a simplification, for practical purposes the FSC signal is often referred to as the size of a particle.

SSC:

SSC refers to "side scatter" and measures scattering of laser light at a $90^{\circ}$-angle. The SSC signal is related to the internal complexity of a particle. For example, a cell that contains a lot of internal membranes, such as a granulocyte, scatters more light at a $90^{\circ}$-angle than a comparable particle of less internal complexity.

\section{FLUORESCENCE:}

Different fluorescence signals can be measured and are described by the same parameters of area, width and height as illustrated above. 


\section{CONTOUR PLOTS:}

In this work I represent the flow-cytometry data in two parameter contour plots (see Figure $10 \mathrm{~A}$ and $\mathrm{D}$ for example). The advantage of using contour plots is that they provide information about the distribution of the data in addition to the signal intensity which is indicated on the $x$ - and $y$ - axes. In the presented work, contour lines represent levels of linear-density, which indicate the proportion of data in different signal ranges. Additionally the different contour levels are colorcoded. (See below for a schematic illustration). The spacing of the contour lines was set to $15 \%$. The outermost contour represents data with a density of $7.5 \%$ of the maximal data density, while the innermost contour represents data at 82.5 $\%$ of the maximal data density. The outliers, meaning events that did not fall within one of the contour levels, are represented as dots.

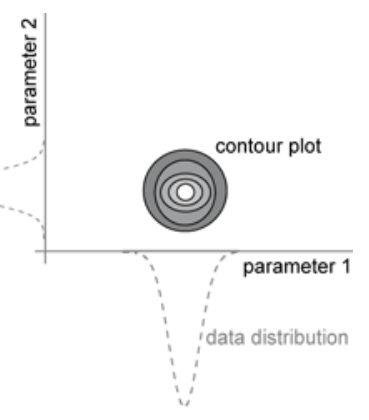

GATES:

Gates are used in flow-cytometry and flow-sorting to select data/events of certain signal ranges for further analyses or sorting. In the presented work I have assigned comprehensible names for different gates used in analyses and sorting. These gate names, such as "small singles" gate are indicated in the figures and highlighted throughout the text by the use quotation marks. 


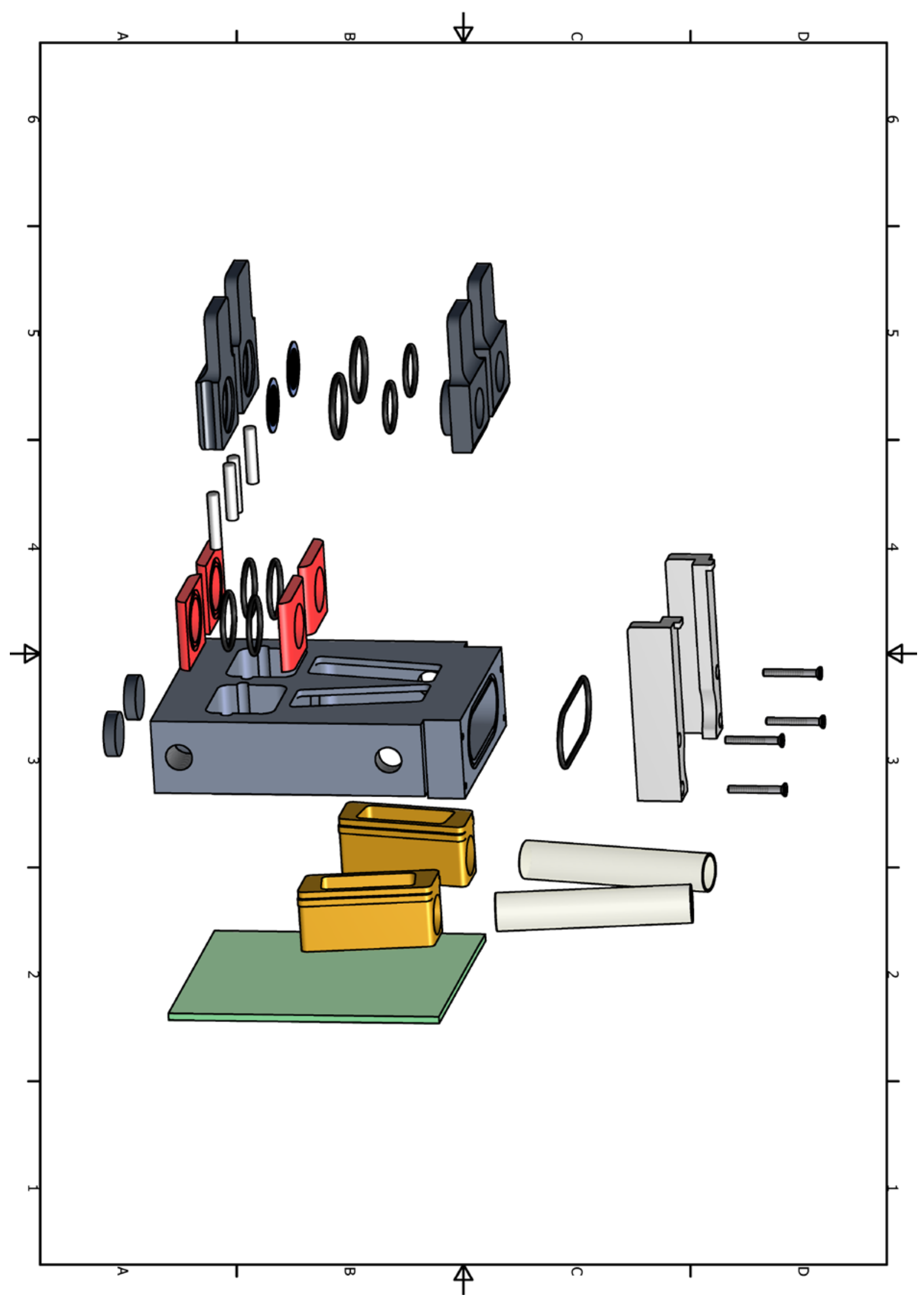

Appendix 2: Water-cooled online-filtration device for FASS 


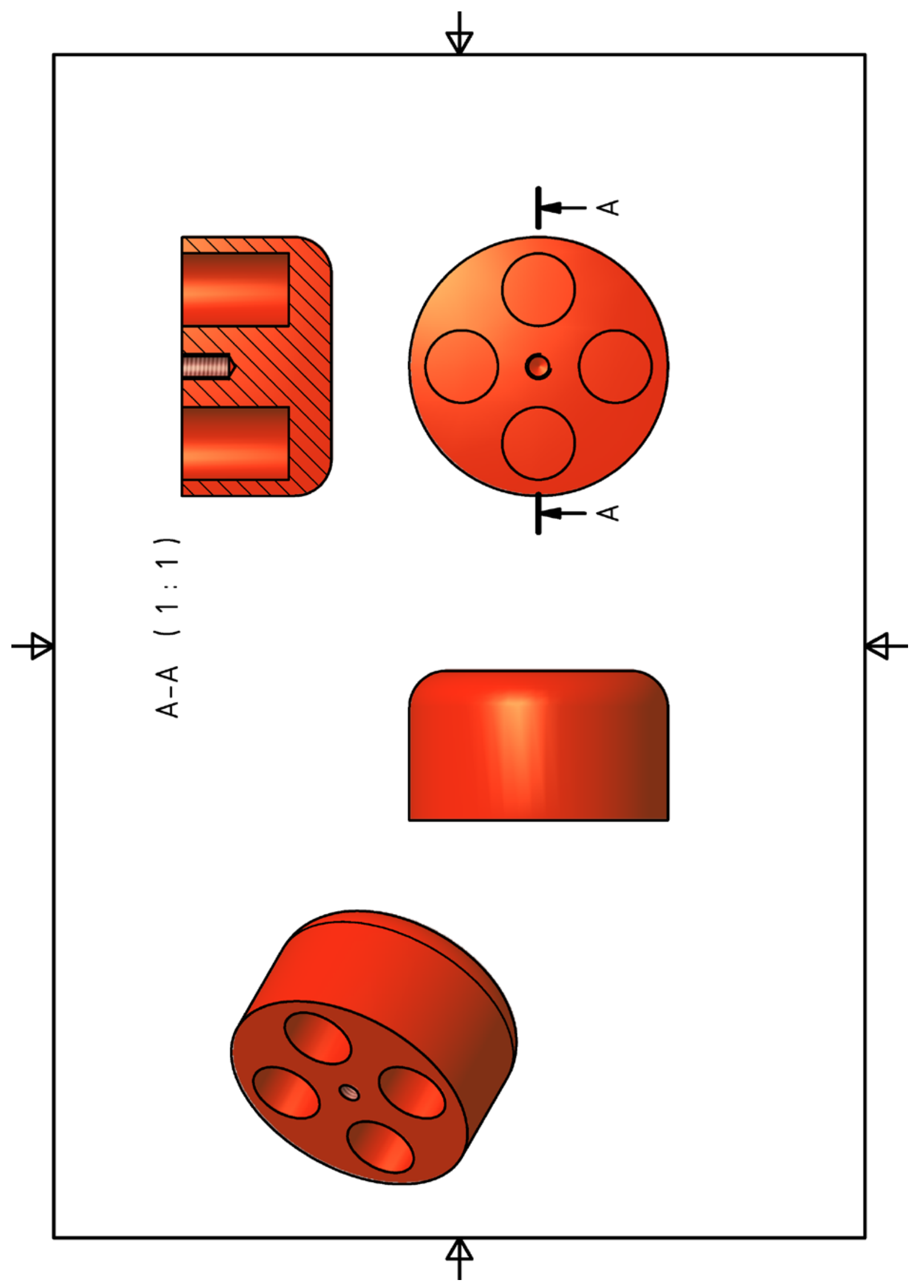

Appendix 3: Centrifuge adaptors for pelleting of synaptosomes onto coverslips after FASS 


\section{Bibliography}

Abul-Husn, N.S., and L.A. Devi. 2006. Neuroproteomics of the synapse and drug addiction. J Pharmacol Exp Ther. 318:461-8.

Agrawal, S., A. Kumar, V. Srivastava, and B.N. Mishra. 2003. Cloning, expression, activity and folding studies of serine hydroxymethyltransferase: a target enzyme for cancer chemotherapy. $J$ Mol Microbiol Biotechnol. 6:67-75.

Al-Hallaq, R.A., R.P. Yasuda, and B.B. Wolfe. 2001. Enrichment of N-methyl-D-aspartate NR1 splice variants and synaptic proteins in rat postsynaptic densities. J Neurochem. 77:110-9.

Al-Shahrour, F., J. Carbonell, P. Minguez, S. Goetz, A. Conesa, J. Tárraga, I. Medina, E. Alloza, D. Montaner, and J. Dopazo. 2008. Babelomics: advanced functional profiling of transcriptomics, proteomics and genomics experiments. Nucleic Acids Res. 36:W341-6.

Aoki, C., C. Venkatesan, C.G. Go, R. Forman, and H. Kurose. 1998. Cellular and subcellular sites for noradrenergic action in the monkey dorsolateral prefrontal cortex as revealed by the immunocytochemical localization of noradrenergic receptors and axons. Cereb Cortex. 8:269-77.

Apostolopoulos, J., L.J. Chisholm, and M.S. Sandrin. 1999. Identification of mouse Ly6H and its expression in normal tissue. Immunogenetics. 49:987-90.

Appaji Rao, N., M. Ambili, V.R. Jala, H.S. Subramanya, and H.S. Savithri. 2003. Structure-function relationship in serine hydroxymethyltransferase. Biochim Biophys Acta. 1647:24-9.

Arendt, T. 2009. Synaptic degeneration in Alzheimer's disease. Acta Neuropathol. 118:167-79.

Arystarkhova, E., C. Donnet, N.K. Asinovski, and K.J. Sweadner. 2002. Differential regulation of renal Na,K-ATPase by splice variants of the gamma subunit. J Biol Chem. 277:10162-72.

Association, A.P. 1994. Diagnostic and Statistical Manual of Mental Disorders. American Psychiatric Publishing Inc. Washington.

Bai, F., and F.A. Witzmann. 2007. Synaptosome proteomics. Subcell Biochem. 43:77-98.

Bajjalieh, S.M., G.D. Frantz, J.M. Weimann, S.K. McConnell, and R.H. Scheller. 1994. Differential expression of synaptic vesicle protein 2 (SV2) isoforms. J Neurosci. 14:5223-35.

Bak, L.K., A. Schousboe, and H.S. Waagepetersen. 2006. The glutamate/GABA-glutamine cycle: aspects of transport, neurotransmitter homeostasis and ammonia transfer. Journal of Neurochemistry. 98:641-53.

Barañano, D.E., C.D. Ferris, and S.H. Snyder. 2001. Atypical neural messengers. Trends Neurosci. 24:99-106.

Bayés, A., and S.G.N. Grant. 2009. Neuroproteomics: understanding the molecular organization and complexity of the brain. Nat Rev Neurosci. 10:635-46.

Béguin, P., G. Crambert, F. Monnet-Tschudi, M. Uldry, J.-D. Horisberger, H. Garty, and K. Geering. 2002. FXYD7 is a brain-specific regulator of Na,K-ATPase alpha 1-beta isozymes. EMBO J. 21:3264-73.

Bellocchio, E.E., H. Hu, A. Pohorille, J. Chan, V.M. Pickel, and R.H. Edwards. 1998. The localization of the brain-specific inorganic phosphate transporter suggests a specific presynaptic role in glutamatergic transmission. J Neurosci. 18:8648-59.

Bellocchio, E.E., R.J. Reimer, R.T. Fremeau, and R.H. Edwards. 2000. Uptake of glutamate into synaptic vesicles by an inorganic phosphate transporter. Science. 289:957-60.

Betz, H., J. Gomeza, W. Armsen, P. Scholze, and V. Eulenburg. 2006. Glycine transporters: essential regulators of synaptic transmission. Biochem Soc Trans. 34:55-8.

Blum, H., H. Beier, and H. Gross. 1987. Improved silver staining of plant proteins, RNA and DNA in polyacrylamide gels. Electrophoresis. 8:93-99.

Böck, G., P. Steinlein, and L.A. Huber. 1997. Cell biologists sort things out: Analysis and purification of intracellular organelles by flow cytometry. Trends Cell Biol. 7:499-503.

Bonvento, G., E.T. MacKenzie, J. Seylaz, and P. Lacombe. 1994. The cerebrovascular role of the ascending serotonergic system: new vistas. J Auton Nerv Syst. 49 Suppl:S37-42.

Boulland, J.-L., T. Qureshi, R.P. Seal, A. Rafiki, V. Gundersen, L.H. Bergersen, R.T. Fremeau, R.H. Edwards, J. Storm-Mathisen, and F.A. Chaudhry. 2004. Expression of the vesicular glutamate transporters during development indicates the widespread corelease of multiple neurotransmitters. J Comp Neurol. 480:264-80.

Boutros, R., S. Fanayan, M. Shehata, and J.A. Byrne. 2004. The tumor protein D52 family: many pieces, many puzzles. Biochem Biophys Res Commun. 325:1115-21. 
Bradford, H.F., M. Docherty, J.Y. Wu, C.D. Cash, M. Ehret, M. Maitre, and T.H. Joh. 1989. The immunolysis, isolation, and properties of subpopulations of mammalian brain synaptosomes. Neurochem Res. 14:301-10.

Bradford, M.M. 1976. A rapid and sensitive method for the quantitation of microgram quantities of protein utilizing the principle of protein-dye binding. Anal Biochem. 72:248-54.

Bragina, L., C. Candiracci, P. Barbaresi, S. Giovedì, F. Benfenati, and F. Conti. 2007. Heterogeneity of glutamatergic and GABAergic release machinery in cerebral cortex. Neuroscience. 146:1829-40.

Brodal, P. 2010. The Central Nervous System: Struc

.591.

Brose, N. 1999. Synaptic cell adhesion proteins and synaptogenesis in the mammalian central nervous system. Naturwissenschaften. 86:516-24.

Brose, N. 2008. For better or for worse: complexins regulate SNARE function and vesicle fusion. Traffic. 9:1403-13.

Brose, N., V. O'Connor, and P. Skehel. 2010. Synaptopathy: dysfunction of synaptic function? Biochem Soc Trans. 38:443-4.

Bu, D.F., M.G. Erlander, B.C. Hitz, N.J. Tillakaratne, D.L. Kaufman, C.B. Wagner-McPherson, G.A. Evans, and A.J. Tobin. 1992. Two human glutamate decarboxylases, 65-kDa GAD and $67-\mathrm{kDa}$ GAD, are each encoded by a single gene. Proceedings of the National Academy of Sciences of the United States of America. 89:2115-9.

Buddhala, C., C.-C. Hsu, and J.-Y. Wu. 2009. A novel mechanism for GABA synthesis and packaging into synaptic vesicles. Neurochem Int. 55:9-12.

Bylund, D.B., R.A. Bond, D.C. Eikenburg, J.P. Hieble, R. Hills, K.P. Minneman, and S. Parra. 2010. IUPHAR_DATABASE_Adrenoceptors_INTRODUCTION. IUPHAR database:1-7.

Byrne, J.A., M.G. Mattei, and P. Basset. 1996. Definition of the tumor protein D52 (TPD52) gene family through cloning of D52 homologues in human (hD53) and mouse (mD52). Genomics. 35:523-32.

Cahoy, J.D., B. Emery, A. Kaushal, L.C. Foo, J.L. Zamanian, K.S. Christopherson, Y. Xing, J.L. Lubischer, P.A. Krieg, S.A. Krupenko, W.J. Thompson, and B.A. Barres. 2008. A transcriptome database for astrocytes, neurons, and oligodendrocytes: a new resource for understanding brain development and function. J Neurosci. 28:264-78.

Cai, Q., and Z.-H. Sheng. 2009. Mitochondrial transport and docking in axons. Exp Neurol. 218:25767.

Chew, C.S., X. Chen, H. Zhang, E.A. Berg, and H. Zhang. 2008. Calcium/calmodulin-dependent phosphorylation of tumor protein D52 on serine residue 136 may be mediated by CAMK2delta6. Am J Physiol Gastrointest Liver Physiol. 295:G1159-72.

Christgau, S., H.J. Aanstoot, H. Schierbeck, K. Begley, S. Tullin, K. Hejnaes, and S. Baekkeskov. 1992. Membrane anchoring of the autoantigen GAD65 to microvesicles in pancreatic beta-cells by palmitoylation in the NH2-terminal domain. The Journal of Cell Biology. 118:309-20.

CLEUGH, J., J.H. GADDUM, A.A. MITCHELL, M.W. SMITH, and V.P. Whittaker. 1964. SUBSTANCE P IN BRAIN EXTRACTS. The Journal of Physiology. 170:69-85.

Colonnier, M. 1968. Synaptic patterns on different cell types in the different laminae of the cat visual cortex. An electron microscope study. Brain Res. 9:268-87.

Cotman, C., H. Herschman, and D. Taylor. 1971. Subcellular fractionation of cultured glial cells. J Neurobiol. 2:169-80.

Coughenour, H.D., R.S. Spaulding, and C.M. Thompson. 2004. The synaptic vesicle proteome: a comparative study in membrane protein identification. Proteomics. 4:3141-55.

Cowan, C.M., and L.A. Raymond. 2006. Selective neuronal degeneration in Huntington's disease. Curr Top Dev Biol. 75:25-71.

Crowder, K.M., J.M. Gunther, T.A. Jones, B.D. Hale, H.Z. Zhang, M.R. Peterson, R.H. Scheller, C. Chavkin, and S.M. Bajjalieh. 1999. Abnormal neurotransmission in mice lacking synaptic vesicle protein 2A (SV2A). Proc Natl Acad Sci USA. 96:15268-73.

Cubelos, B., C. Giménez, and F. Zafra. 2005a. Localization of the GLYT1 glycine transporter at glutamatergic synapses in the rat brain. Cereb Cortex. 15:448-59.

Cubelos, B., I.M. González-González, C. Giménez, and F. Zafra. 2005b. The scaffolding protein PSD95 interacts with the glycine transporter GLYT1 and impairs its internalization. J Neurochem. 95:1047-58.

Danbolt, N.C. 2001. Glutamate uptake. Prog Neurobiol. 65:1-105.

Darvas, M., M. Morsch, I. Racz, S. Ahmadi, D. Swandulla, and A. Zimmer. 2009. Modulation of the $\mathrm{Ca} 2+$ conductance of nicotinic acetylcholine receptors by Lypd6. Eur Neuropsychopharmacol. 19:670-81. 
De Camilli, P., R. Cameron, and P. Greengard. 1983. Synapsin I (protein I), a nerve terminal-specific phosphoprotein. I. Its general distribution in synapses of the central and peripheral nervous system demonstrated by immunofluorescence in frozen and plastic sections. $J$ Cell Biol. 96:1337-54.

De Gois, S., E. Jeanclos, M. Morris, S. Grewal, H. Varoqui, and J.D. Erickson. 2006. Identification of endophilins 1 and 3 as selective binding partners for VGLUT1 and their co-localization in neocortical glutamatergic synapses: implications for vesicular glutamate transporter trafficking and excitatory vesicle formation. Cell Mol Neurobiol. 26:679-93.

De Pinto, V., A. Messina, D.J.R. Lane, and A. Lawen. 2010. Voltage-dependent anion-selective channel (VDAC) in the plasma membrane. FEBS Letters.

Delprat, B., J.-L. Puel, and K. Geering. 2007a. Dynamic expression of FXYD6 in the inner ear suggests a role of the protein in endolymph homeostasis and neuronal activity. Dev Dyn. 236:2534-40.

Delprat, B., D. Schaer, S. Roy, J. Wang, J.-L. Puel, and K. Geering. 2007b. FXYD6 is a novel regulator of $\mathrm{Na}, \mathrm{K}-\mathrm{ATP}$ ase expressed in the inner ear. J Biol Chem. 282:7450-6.

Dessaud, E., D. Salaün, O. Gayet, M. Chabbert, and O. deLapeyrière. 2006. Identification of lynx2, a novel member of the ly-6/neurotoxin superfamily, expressed in neuronal subpopulations during mouse development. Mol Cell Neurosci. 31:232-42.

Dirkx, R., A. Thomas, L. Li, A. Lernmark, R.S. Sherwin, P. De Camilli, and M. Solimena. 1995. Targeting of the 67-kDa isoform of glutamic acid decarboxylase to intracellular organelles is mediated by its interaction with the $\mathrm{NH} 2$-terminal region of the 65-kDa isoform of glutamic acid decarboxylase. J Biol Chem. 270:2241-6.

Dobbertin, A., A. Hrabovska, K. Dembele, S. Camp, P. Taylor, E. Krejci, and V. Bernard. 2009. Targeting of acetylcholinesterase in neurons in vivo: a dual processing function for the proline-rich membrane anchor subunit and the attachment domain on the catalytic subunit. J Neurosci. 29:4519-30.

Docherty, M., H.F. Bradford, B. Anderton, and J.Y. Wu. 1983. Specific lysis of GABAergic synaptosomes by an antiserum to glutamate decarboxylase. FEBS Lett. 152:57-61.

Docherty, M., H.F. Bradford, C.D. Cash, M. Ehret, M. Maitre, and T.H. Joh. 1991. Isolation of monoaminergic synaptosomes from rat brain by immunomagnetophoresis. $\mathrm{J}$ Neurochem. 56:1569-80.

Docherty, M., H.F. Bradford, and J.Y. Wu. 1987. The preparation of highly purified GABAergic and cholinergic synaptosomes from mammalian brain. Neurosci Lett. 81:232-8.

Docherty, M., H.F. Bradford, J.Y. Wu, T.H. Joh, and D.J. Reis. 1985. Evidence for specific immunolysis of nerve terminals using antisera against choline acetyltransferase, glutamate decarboxylase and tyrosine hydroxylase. Brain Res. 339:105-13.

Dodd, P.R., J.A. Hardy, A.E. Oakley, J.A. Edwardson, E.K. Perry, and J.P. Delaunoy. 1981. A rapid method for preparing synaptosomes: comparison, with alternative procedures. Brain Res. 226:107-18.

Dunkley, P.R., P.E. Jarvie, J.W. Heath, G.J. Kidd, and J.A. Rostas. 1986. A rapid method for isolation of synaptosomes on Percoll gradients. Brain Res. 372:115-29.

E. Celis, J. 1998. Cell Biology: A Laboratory Handbook - Page 102.674.

Eastwood, S.L., and P.J. Harrison. 2005. Decreased expression of vesicular glutamate transporter 1 and complexin II mRNAs in schizophrenia: further evidence for a synaptic pathology affecting glutamate neurons. Schizophr Res. 73:159-72.

Eiden, L.E., M.K.-H. Schäfer, E. Weihe, and B. Schütz. 2004. The vesicular amine transporter family (SLC18): amine/proton antiporters required for vesicular accumulation and regulated exocytotic secretion of monoamines and acetylcholine. Pflugers Arch. 447:636-40.

Eisenbarth, G.S., F.S. Walsh, and M. Nirenberg. 1979. Monoclonal antibody to a plasma membrane antigen of neurons. Proc Natl Acad Sci USA. 76:4913-7.

Evergren, E., F. Benfenati, and O. Shupliakov. 2007. The synapsin cycle: a view from the synaptic endocytic zone. J Neurosci Res. 85:2648-56.

Fan, M.M.Y., H.B. Fernandes, L.Y.J. Zhang, M.R. Hayden, and L.A. Raymond. 2007. Altered NMDA receptor trafficking in a yeast artificial chromosome transgenic mouse model of Huntington's disease. J Neurosci. 27:3768-79.

Farrant, M., and K. Kaila. 2007. The cellular, molecular and ionic basis of GABA(A) receptor signalling. Prog Brain Res. 160:59-87.

Fein, J.A., S. Sokolow, C.A. Miller, H.V. Vinters, F. Yang, G.M. Cole, and K.H. Gylys. 2008. Colocalization of amyloid beta and tau pathology in Alzheimer's disease synaptosomes. Am J Pathol. 172:1683-92.

Fernández-Chacón, R., and T.C. Südhof. 1999. Genetics of synaptic vesicle function: toward the complete functional anatomy of an organelle. Annu Rev Physiol. 61:753-76. 
Fremeau, R.T., J. Burman, T. Qureshi, C.H. Tran, J. Proctor, J. Johnson, H. Zhang, D. Sulzer, D.R. Copenhagen, J. Storm-Mathisen, R.J. Reimer, F.A. Chaudhry, and R.H. Edwards. 2002. The identification of vesicular glutamate transporter 3 suggests novel modes of signaling by glutamate. Proc Natl Acad Sci U S A. 99:14488-93.

Fremeau, R.T., K. Kam, T. Qureshi, J. Johnson, D.R. Copenhagen, J. Storm-Mathisen, F.A. Chaudhry, R.A. Nicoll, and R.H. Edwards. 2004a. Vesicular glutamate transporters 1 and 2 target to functionally distinct synaptic release sites. Science. 304:1815-9.

Fremeau, R.T., M.D. Troyer, I. Pahner, G.O. Nygaard, C.H. Tran, R.J. Reimer, E.E. Bellocchio, D. Fortin, J. Storm-Mathisen, and R.H. Edwards. 2001. The expression of vesicular glutamate transporters defines two classes of excitatory synapse. Neuron. 31:247-60.

Fremeau, R.T., S. Voglmaier, R.P. Seal, and R.H. Edwards. 2004b. VGLUTs define subsets of excitatory neurons and suggest novel roles for glutamate. Trends Neurosci. 27:98-103.

Gaffield, M.A., and W.J. Betz. 2006. Imaging synaptic vesicle exocytosis and endocytosis with FM dyes. Nature protocols. 1:2916-21.

Garty, H., and S.J.D. Karlish. 2006. Role of FXYD proteins in ion transport. Annu Rev Physiol. 68:43159.

Geering, K. 2006. FXYD proteins: new regulators of Na-K-ATPase. AJP - Renal Physiology. 290:F241-50.

Giepmans, B.N.G., S.R. Adams, M.H. Ellisman, and R.Y. Tsien. 2006. The fluorescent toolbox for assessing protein location and function. Science. 312:217-24.

Giros, B., M. Jaber, S.R. Jones, R.M. Wightman, and M.G. Caron. 1996. Hyperlocomotion and indifference to cocaine and amphetamine in mice lacking the dopamine transporter. Nature. 379:606-12.

Gomeza, J., S. Hülsmann, K. Ohno, V. Eulenburg, K. Szöke, D. Richter, and H. Betz. 2003. Inactivation of the glycine transporter 1 gene discloses vital role of glial glycine uptake in glycinergic inhibition. Neuron. 40:785-96.

Gras, C., B. Amilhon, E.M. Lepicard, O. Poirel, J. Vinatier, M. Herbin, S. Dumas, E.T. Tzavara, M.R. Wade, G.G. Nomikos, N. Hanoun, F. Saurini, M.-L. Kemel, B. Gasnier, B. Giros, and S. El Mestikawy. 2008. The vesicular glutamate transporter VGLUT3 synergizes striatal acetylcholine tone. Nat Neurosci. 11:292-300.

Gras, C., E. Herzog, G.C. Bellenchi, V. Bernard, P. Ravassard, M. Pohl, B. Gasnier, B. Giros, and S. El Mestikawy. 2002. A third vesicular glutamate transporter expressed by cholinergic and serotoninergic neurons. J Neurosci. 22:5442-51.

Gray, E.G. 1959. Electron microscopy of synaptic contacts on dendrite spines of the cerebral cortex. Nature. 183:1592-3.

Greer, J.M., and M.B. Lees. 2002. Myelin proteolipid protein--the first 50 years. Int J Biochem Cell Biol. 34:211-5.

Groblewski, G.E., M.J. Wishart, M. Yoshida, and J.A. Williams. 1996. Purification and identification of a $28-\mathrm{kDa}$ calcium-regulated heat-stable protein. A novel secretagogue-regulated phosphoprotein in exocrine pancreas. J Biol Chem. 271:31502-7.

Groblewski, G.E., M. Yoshida, H. Yao, J.A. Williams, and S.A. Ernst. 1999. Immunolocalization of CRHSP28 in exocrine digestive glands and gastrointestinal tissues of the rat. $A m \mathrm{~J}$ Physiol. 276:G219-26.

Groc, L., L. Bard, and D. Choquet. 2009. Surface trafficking of N-methyl-D-aspartate receptors: physiological and pathological perspectives. Neuroscience. 158:4-18.

Groc, L., M. Heine, L. Cognet, K. Brickley, F.A. Stephenson, B. Lounis, and D. Choquet. 2004. Differential activity-dependent regulation of the lateral mobilities of AMPA and NMDA receptors. Nat Neurosci. 7:695-6.

Groc, L., M. Heine, S.L. Cousins, F.A. Stephenson, B. Lounis, L. Cognet, and D. Choquet. 2006. NMDA receptor surface mobility depends on NR2A-2B subunits. Proc Natl Acad Sci USA. 103:18769-74.

Grønborg, M., N.J. Pavlos, I. Brunk, J.J.E. Chua, A. Münster-Wandowski, D. Riedel, G. Ahnert-Hilger, $\mathrm{H}$. Urlaub, and R. Jahn. 2010. Quantitative comparison of glutamatergic and GABAergic synaptic vesicles unveils selectivity for few proteins including MAL2, a novel synaptic vesicle protein. J Neurosci. 30:2-12.

Group, T.H.s.D.C.R. 1993. A novel gene containing a trinucleotide repeat that is expanded and unstable on Huntington's disease chromosomes. The Huntington's Disease Collaborative Research Group. Cell. 72:971-83.

Gundersen, V., N.C. Danbolt, O.P. Ottersen, and J. Storm-Mathisen. 1993. Demonstration of glutamate/aspartate uptake activity in nerve endings by use of antibodies recognizing exogenous D-aspartate. Neuroscience. 57:97-111. 
Gylys, K.H., J.A. Fein, and G.M. Cole. 2000. Quantitative characterization of crude synaptosomal fraction (P-2) components by flow cytometry. J Neurosci Res. 61:186-92.

Gylys, K.H., J.A. Fein, A.M. Tan, and G.M. Cole. 2003. Apolipoprotein E enhances uptake of soluble but not aggregated amyloid-beta protein into synaptic terminals. Journal of Neurochemistry. 84:1442-51.

Gylys, K.H., J.A. Fein, F. Yang, and G.M. Cole. 2004a. Enrichment of presynaptic and postsynaptic markers by size-based gating analysis of synaptosome preparations from rat and human cortex. Cytometry A. 60:90-6.

Gylys, K.H., J.A. Fein, F. Yang, C.A. Miller, and G.M. Cole. 2007. Increased cholesterol in Abetapositive nerve terminals from Alzheimer's disease cortex. Neurobiol Aging. 28:8-17.

Gylys, K.H., J.A. Fein, F. Yang, D.J. Wiley, C.A. Miller, and G.M. Cole. 2004b. Synaptic changes in Alzheimer's disease: increased amyloid-beta and gliosis in surviving terminals is accompanied by decreased PSD-95 fluorescence. Am J Pathol. 165:1809-17.

Harris, A.Z., and D.L. Pettit. 2007. Extrasynaptic and synaptic NMDA receptors form stable and uniform pools in rat hippocampal slices. The Journal of Physiology. 584:509-19.

Harrison, P.J., and S.L. Eastwood. 1998. Preferential involvement of excitatory neurons in medial temporal lobe in schizophrenia. Lancet. 352:1669-73.

Hebb, C., and V. Whittaker. 1958. Intracellular distributions of acetylcholine and choline acetylase. The Journal of Physiology. 142:187-196.

Henn, F.A., D.J. Anderson, and D.G. Rustad. 1976. Glial contamination of synaptosomal fractions. Brain Res. 101:341-4.

Hernandes, M.S., and L.R.P. Troncone. 2009. Glycine as a neurotransmitter in the forebrain: a short review. J Neural Transm. 116:1551-60.

Herrera, G., L. Diaz, A. Martinez-Romero, A. Gomes, E. Villamón, R.C. Callaghan, and J.-E. O'Connor. 2007. Cytomics: A multiparametric, dynamic approach to cell research. Toxicol In Vitro. 21:176-82.

Hertz, L., Y. Chen, M.E. Gibbs, P. Zang, and L. Peng. 2004. Astrocytic adrenoceptors: a major drug target in neurological and psychiatric disorders? Curr Drug Targets CNS Neurol Disord. 3:239-67.

Herzog, E. 2003. Caracterisation des transporteurs vesiculaires du glutamate et diversite des systemes glutamatergiques dans le cerveau de rat. PhD Thesis:1-127.

Herzog, E., G.C. Bellenchi, C. Gras, V. Bernard, P. Ravassard, C. Bedet, B. Gasnier, B. Giros, and S. El Mestikawy. 2001. The existence of a second vesicular glutamate transporter specifies subpopulations of glutamatergic neurons. J Neurosci. 21:RC181.

Herzog, E., J. Gilchrist, C. Gras, A. Muzerelle, P. Ravassard, B. Giros, P. Gaspar, and S. El Mestikawy. 2004a. Localization of VGLUT3, the vesicular glutamate transporter type 3, in the rat brain. Neuroscience. 123:983-1002.

Herzog, E., M. Landry, E. Buhler, R. Bouali-Benazzouz, C. Legay, C.E. Henderson, F. Nagy, P. Dreyfus, B. Giros, and S. El Mestikawy. 2004b. Expression of vesicular glutamate transporters, VGLUT1 and VGLUT2, in cholinergic spinal motoneurons. Eur J Neurosci. 20:1752-60.

Hoffman, R.A. 2005. Standardization, calibration, and control in flow cytometry. Curr Protoc Cytom. Chapter 1:Unit 1.3.

Holford, M., S. Auer, M. Laqua, and I. Ibañez-Tallon. 2009. Manipulating neuronal circuits with endogenous and recombinant cell-surface tethered modulators. Front Mol Neurosci. 2:21.

Holmes, A., J.E. Lachowicz, and D.R. Sibley. 2004. Phenotypic analysis of dopamine receptor knockout mice; recent insights into the functional specificity of dopamine receptor subtypes. Neuropharmacology. 47:1117-34.

Holt, M., F. Varoqueaux, K. Wiederhold, S. Takamori, H. Urlaub, D. Fasshauer, and R. Jahn. 2006. Identification of SNAP-47, a novel Qbc-SNARE with ubiquitous expression. J Biol Chem. 281:17076-83.

Horie, M., K. Okutomi, Y. Taniguchi, Y. Ohbuchi, M. Suzuki, and E. Takahashi. 1998. Isolation and characterization of a new member of the human Ly6 gene family (LY6H). Genomics. 53:365-8.

Huttner, W.B., W. Schiebler, P. Greengard, and P. De Camilli. 1983. Synapsin I (protein I), a nerve terminal-specific phosphoprotein. III. Its association with synaptic vesicles studied in a highly purified synaptic vesicle preparation. J Cell Biol. 96:1374-88.

Icard-Liepkalns, C., S. Berrard, N. Faucon Biguet, B. Lebourdelles, P. Ravassard, J.J. Robert, and J. Mallet. 1993. Tyrosine hydroxylase regulation in neurotransmission and neuroplasticity. $J$ Physiol Paris. 87:153-7. 
Imai, Y., I. Ibata, D. Ito, K. Ohsawa, and S. Kohsaka. 1996. A novel gene iba1 in the major histocompatibility complex class III region encoding an EF hand protein expressed in a monocytic lineage. Biochem Biophys Res Commun. 224:855-62.

Israël, M., and V.P. Whittaker. 1965. The isolation of mossy fibre endings from the granular layer of the cerebellar cortex. Experientia. 21:325-6.

Ivanov, A., C. Pellegrino, S. Rama, I. Dumalska, Y. Salyha, Y. Ben-Ari, and I. Medina. 2006. Opposing role of synaptic and extrasynaptic NMDA receptors in regulation of the extracellular signalregulated kinases (ERK) activity in cultured rat hippocampal neurons. The Journal of Physiology. 572:789-98.

Jahn, R., and R.H. Scheller. 2006. SNAREs--engines for membrane fusion. Nat Rev Mol Cell Biol. 7:631-43.

Jamain, S., H. Quach, C. Betancur, M. Råstam, C. Colineaux, I.C. Gillberg, H. Soderstrom, B. Giros, M. Leboyer, C. Gillberg, T. Bourgeron, and P.A.R.I.S. Study. 2003. Mutations of the Xlinked genes encoding neuroligins NLGN3 and NLGN4 are associated with autism. Nat Genet. 34:27-9.

Jamain, S., K. Radyushkin, K. Hammerschmidt, S. Granon, S. Boretius, F. Varoqueaux, N. Ramanantsoa, J. Gallego, A. Ronnenberg, D. Winter, J. Frahm, J. Fischer, T. Bourgeron, $\mathrm{H}$. Ehrenreich, and N. Brose. 2008. Reduced social interaction and ultrasonic communication in a mouse model of monogenic heritable autism. Proc Natl Acad Sci USA. 105:1710-5.

Janz, R., Y. Goda, M. Geppert, M. Missler, and T.C. Südhof. 1999. SV2A and SV2B function as redundant $\mathrm{Ca} 2+$ regulators in neurotransmitter release. Neuron. 24:1003-16.

Ji, B., Z. Zhang, M. Zhang, H. Zhu, K. Zhou, J. Yang, Y. Li, L. Sun, G. Feng, Y. Wang, L. He, and C. Wan. 2009. Differential expression profiling of the synaptosome proteome in a rat model of antipsychotic resistance. Brain Res. 1295:170-8.

Jin, H., H. Wu, G. Osterhaus, J. Wei, K. Davis, D. Sha, E. Floor, C.-C. Hsu, R.D. Kopke, and J.-Y. Wu. 2003. Demonstration of functional coupling between gamma -aminobutyric acid (GABA) synthesis and vesicular GABA transport into synaptic vesicles. Proceedings of the National Academy of Sciences of the United States of America. 100:4293-8.

Kadowaki, K., K. Sugimoto, F. Yamaguchi, T. Song, Y. Watanabe, K. Singh, and M. Tokuda. 2004. Phosphohippolin expression in the rat central nervous system. Brain Res Mol Brain Res. 125:105-12.

Kaneko, T., F. Fujiyama, and H. Hioki. 2002. Immunohistochemical localization of candidates for vesicular glutamate transporters in the rat brain. J Comp Neurol. 444:39-62.

Kanner, B.I., and S. Schuldiner. 1987. Mechanism of transport and storage of neurotransmitters. CRC Crit Rev Biochem. 22:1-38.

Kashani, Lepicard, Poirel, Videau, David, Fallet-Bianco, Simon, Delacourte, Giros, Epelbaum, Betancur, and E. Mestikawy. 2007. Loss of VGLUT1 and VGLUT2 in the prefrontal cortex is correlated with cognitive decline in Alzheimer disease. Neurobiol Aging.

Laemmli, U.K. 1970. Cleavage of structural proteins during the assembly of the head of bacteriophage T4. Nature. 227:680-5.

Lakso, M., J.G. Pichel, J.R. Gorman, B. Sauer, Y. Okamoto, E. Lee, F.W. Alt, and H. Westphal. 1996. Efficient in vivo manipulation of mouse genomic sequences at the zygote stage. Proc Natl Acad Sci USA. 93:5860-5.

Lan, C.T., C.Y. Wen, C.K. Tan, E.A. Ling, and J.Y. Shieh. 1995. Ultrastructural identification of cholinergic neurons in the external cuneate nucleus of the gerbil: acetylcholinesterase histochemistry and choline acetyltransferase immunocytochemistry. J Neurocytol. 24:83852.

Lee, S.H., J.G. Valtschanoff, V.N. Kharazia, R. Weinberg, and M. Sheng. 2001a. Biochemical and morphological characterization of an intracellular membrane compartment containing AMPA receptors. Neuropharmacology. 41:680-92.

Lee, V.M., M. Goedert, and J.Q. Trojanowski. 2001b. Neurodegenerative tauopathies. Annu Rev Neurosci. 24:1121-59.

Legendre, P. 2001. The glycinergic inhibitory synapse. Cell Mol Life Sci. 58:760-93.

Lehre, K.P., and N.C. Danbolt. 1998. The number of glutamate transporter subtype molecules at glutamatergic synapses: chemical and stereological quantification in young adult rat brain. J Neurosci. 18:8751-7.

Lisé, M.-F., and A. El-Husseini. 2006. The neuroligin and neurexin families: from structure to function at the synapse. Cell Mol Life Sci. 63:1833-49.

Liu, H., R.G. Sadygov, and J.R. Yates. 2004. A model for random sampling and estimation of relative protein abundance in shotgun proteomics. Anal Chem. 76:4193-201. 
Lotharius, J., and P. Brundin. 2002. Pathogenesis of Parkinson's disease: dopamine, vesicles and alpha-synuclein. Nat Rev Neurosci. 3:932-42.

Lundgren, D.H., S.-I. Hwang, L. Wu, and D.K. Han. 2010. Role of spectral counting in quantitative proteomics. Expert Rev Proteomics. 7:39-53.

Luo, S., N.B. Wehr, and R.L. Levine. 2006. Quantitation of protein on gels and blots by infrared fluorescence of Coomassie blue and Fast Green. Anal Biochem. 350:233-8.

Martin, D.L., and K. Rimvall. 1993. Regulation of gamma-aminobutyric acid synthesis in the brain. Journal of Neurochemistry. 60:395-407.

Martínez-Murillo, R., R. Villalba, M.I. Montero-Caballero, and J. Rodrigo. 1989a. Cholinergic somata and terminals in the rat substantia nigra: an immunocytochemical study with optical and electron microscopic techniques. J Comp Neurol. 281:397-415.

Martínez-Murillo, R., R.M. Villalba, and J. Rodrigo. 1989b. Electron microscopic localization of cholinergic terminals in the rat substantia nigra: an immunocytochemical study. Neurosci Lett. 96:121-6.

Masson, J., C. Sagné, M. Hamon, and S. El Mestikawy. 1999. Neurotransmitter transporters in the central nervous system. Pharmacol Rev. 51:439-64.

Maycox, P.R., J.W. Hell, and R. Jahn. 1990. Amino acid neurotransmission: spotlight on synaptic vesicles. Trends Neurosci. 13:83-7.

McIntire, S.L., R.J. Reimer, K. Schuske, R.H. Edwards, and E.M. Jorgensen. 1997. Identification and characterization of the vesicular GABA transporter. Nature. 389:870-6.

McKenna, M.C. 2007. The glutamate-glutamine cycle is not stoichiometric: fates of glutamate in brain. J Neurosci Res. 85:3347-58.

Milnerwood, A.J., C.M. Gladding, M.A. Pouladi, A.M. Kaufman, R.M. Hines, J.D. Boyd, R.W.Y. Ko, O.C. Vasuta, R.K. Graham, M.R. Hayden, T.H. Murphy, and L.A. Raymond. 2010. Early increase in extrasynaptic NMDA receptor signaling and expression contributes to phenotype onset in Huntington's disease mice. Neuron. 65:178-90.

Miwa, J.M., I. Ibanez-Tallon, G.W. Crabtree, R. Sánchez, A. Sali, L.W. Role, and N. Heintz. 1999. lynx1, an endogenous toxin-like modulator of nicotinic acetylcholine receptors in the mammalian CNS. Neuron. 23:105-14.

Miwa, J.M., T.R. Stevens, S.L. King, B.J. Caldarone, I. Ibanez-Tallon, C. Xiao, R.M. Fitzsimonds, C. Pavlides, H.A. Lester, M.R. Picciotto, and N. Heintz. 2006. The prototoxin lynx1 acts on nicotinic acetylcholine receptors to balance neuronal activity and survival in vivo. Neuron. 51:587-600.

Moechars, D., M.C. Weston, S. Leo, Z. Callaerts-Vegh, I. Goris, G. Daneels, A. Buist, M. Cik, P. van der Spek, S. Kass, T. Meert, R. D'Hooge, C. Rosenmund, and R.M. Hampson. 2006. Vesicular glutamate transporter VGLUT2 expression levels control quantal size and neuropathic pain. J Neurosci. 26:12055-66.

Morciano, M., J. Burré, C. Corvey, M. Karas, H. Zimmermann, and W. Volknandt. 2005. Immunoisolation of two synaptic vesicle pools from synaptosomes: a proteomics analysis. J Neurochem. 95:1732-45.

Mortz, E., T. Krogh, H. Vorum, and A. Görg. 2001. Improved silver staining protocols for high sensitivity protein identification using matrix-assisted laser desorption/ionization-time of flight analysis. Proteomics. 1:1359-1363.

Moseley, A.E., S.P. Lieske, R.K. Wetzel, P.F. James, S. He, D.A. Shelly, R.J. Paul, G.P. Boivin, D.P. Witte, J.M. Ramirez, K.J. Sweadner, and J.B. Lingrel. 2003. The Na,K-ATPase alpha 2 isoform is expressed in neurons, and its absence disrupts neuronal activity in newborn mice. J Biol Chem. 278:5317-24.

Mueller, L.N., M.-Y. Brusniak, D.R. Mani, and R. Aebersold. 2008. An assessment of software solutions for the analysis of mass spectrometry based quantitative proteomics data. $J$ Proteome Res. 7:51-61.

Mullis, K., F. Faloona, S. Scharf, R. Saiki, G. Horn, and H. Erlich. 1986. Specific enzymatic amplification of DNA in vitro: the polymerase chain reaction. Cold Spring Harb Symp Quant Biol. 51 Pt 1:263-73.

Nachmansohn, D., and A. Machado. 1943. THE FORMATION OF ACETYLCHOLINE. A NEW ENZYME: "CHOLINE ACETYLASE". J Neurophysiol. 6:397-403.

Nesvizhskii, A.I., A. Keller, E. Kolker, and R. Aebersold. 2003. A statistical model for identifying proteins by tandem mass spectrometry. Anal Chem. 75:4646-58.

Neuhoff, V., N. Arold, D. Taube, and W. Ehrhardt. 1988. Improved staining of proteins in polyacrylamide gels including isoelectric focusing gels with clear background at nanogram sensitivity using Coomassie Brilliant Blue G-250 and R-250. Electrophoresis. 9:255-262. 
Neuhoff, V., R. Stamm, and H. Eibl. 1985. Clear background and highly sensitive protein staining with Coomassie Blue dyes in polyacrylamide gels: A systematic analysis. Electrophoresis. 6:427-448.

Niswender, C.M., and P.J. Conn. 2010. Metabotropic glutamate receptors: physiology, pharmacology, and disease. Annu Rev Pharmacol Toxicol. 50:295-322.

Norde, W. 1992. The behavior of proteins at interfaces, with special attention to the role of the structure stability of the protein molecule. Clinical Materials. 11:85-91.

Oda, Y. 1999. Choline acetyltransferase: the structure, distribution and pathologic changes in the central nervous system. Pathol Int. 49:921-37.

Okabe, S. 2007. Molecular anatomy of the postsynaptic density. Mol Cell Neurosci. 34:503-18.

Okamoto, S.-i., M.A. Pouladi, M. Talantova, D. Yao, P. Xia, D.E. Ehrnhoefer, R. Zaidi, A. Clemente, M. Kaul, R.K. Graham, D. Zhang, H.-S. Vincent Chen, G. Tong, M.R. Hayden, and S.A. Lipton. 2009. Balance between synaptic versus extrasynaptic NMDA receptor activity influences inclusions and neurotoxicity of mutant huntingtin. Nat Med. 15:1407-13.

Oldham, M.C., G. Konopka, K. Iwamoto, P. Langfelder, T. Kato, S. Horvath, and D.H. Geschwind. 2008. Functional organization of the transcriptome in human brain. Nat Neurosci. 11:1271-82.

Ottersen, O.P., F.A. Chaudhry, N.C. Danbolt, J.H. Laake, E.A. Nagelhus, J. Storm-Mathisen, and R. Torp. 1997. Molecular organization of cerebellar glutamate synapses. Prog Brain Res. 114:97-107.

Parameshwaran, K., M. Dhanasekaran, and V. Suppiramaniam. 2008. Amyloid beta peptides and glutamatergic synaptic dysregulation. Exp Neurol. 210:7-13.

Parente, J.A., J.R. Goldenring, A.C. Petropoulos, U. Hellman, and C.S. Chew. 1996. Purification, cloning, and expression of a novel, endogenous, calcium-sensitive, 28-kDa phosphoprotein. J Biol Chem. 271:20096-101.

Patterson, S.D., and R.H. Aebersold. 2003. Proteomics: the first decade and beyond. Nat Genet. 33 Suppl:311-23.

Petralia, R.S., Y.X. Wang, F. Hua, Z. Yi, A. Zhou, L. Ge, F.A. Stephenson, and R.J. Wenthold. 2010. Organization of NMDA receptors at extrasynaptic locations. Neuroscience. 167:68-87.

Pines, G., N.C. Danbolt, M. Bjørås, Y. Zhang, A. Bendahan, L. Eide, H. Koepsell, J. Storm-Mathisen, E. Seeberg, and B.I. Kanner. 1992. Cloning and expression of a rat brain L-glutamate transporter. Nature. 360:464-7.

Ploug, M., and V. Ellis. 1994. Structure-function relationships in the receptor for urokinase-type plasminogen activator. Comparison to other members of the Ly- 6 family and snake venom alpha-neurotoxins. FEBS Lett. 349:163-8.

Ploug, M., M. Kjalke, E. Rønne, U. Weidle, G. Høyer-Hansen, and K. Danø. 1993. Localization of the disulfide bonds in the $\mathrm{NH}$ 2-terminal domain of the cellular receptor for human urokinasetype plasminogen activator. A domain structure belonging to a novel superfamily of glycolipid-anchored membrane proteins. J Biol Chem. 268:17539-46.

Proux-Gillardeaux, V., T. Galli, I. Callebaut, A. Mikhailik, G. Calothy, and M. Marx. 2003. D53 is a novel endosomal SNARE-binding protein that enhances interaction of syntaxin 1 with the synaptobrevin 2 complex in vitro. Biochem J. 370:213-21.

R. Kandel, E., J. H. Schwartz, and T. M. Jessell. 2000. Principles of Neural Science.1414.

Radyushkin, K., K. Hammerschmidt, S. Boretius, F. Varoqueaux, A. El-Kordi, A. Ronnenberg, D. Winter, J. Frahm, J. Fischer, N. Brose, and H. Ehrenreich. 2009. Neuroligin-3-deficient mice: model of a monogenic heritable form of autism with an olfactory deficit. Genes Brain Behav. 8:416-25.

Reim, K., M. Mansour, F. Varoqueaux, H.T. McMahon, T.C. Südhof, N. Brose, and C. Rosenmund. 2001. Complexins regulate a late step in Ca2+-dependent neurotransmitter release. Cell. 104:71-81.

Richards, K.S., K. Bommert, G. Szabo, and R. Miles. 2007. Differential expression of Na+/K+-ATPase alpha-subunits in mouse hippocampal interneurones and pyramidal cells. J Physiol (Lond). 585:491-505.

Rizzoli, S.O., and W.J. Betz. 2005. Synaptic vesicle pools. Nat Rev Neurosci. 6:57-69.

Rosenmund, C., A. Feltz, and G.L. Westbrook. 1995. Synaptic NMDA receptor channels have a low open probability. J Neurosci. 15:2788-95.

Rothstein, J.D., M. Dykes-Hoberg, C.A. Pardo, L.A. Bristol, L. Jin, R.W. Kuncl, Y. Kanai, M.A. Hediger, Y. Wang, J.P. Schielke, and D.F. Welty. 1996. Knockout of glutamate transporters reveals a major role for astroglial transport in excitotoxicity and clearance of glutamate. Neuron. 16:675-86. 
Sagné, C., S. El Mestikawy, M.F. Isambert, M. Hamon, J.P. Henry, B. Giros, and B. Gasnier. 1997. Cloning of a functional vesicular GABA and glycine transporter by screening of genome databases. FEBS Lett. 417:177-83.

Sambrook, J., E. Fritsch, and T. Maniatis. 1989. Molecular cloning: a laboratory manual.

Sasaki, Y.F., T. Rothe, L.S. Premkumar, S. Das, J. Cui, M.V. Talantova, H.-K. Wong, X. Gong, S.F. Chan, D. Zhang, N. Nakanishi, N.J. Sucher, and S.A. Lipton. 2002. Characterization and comparison of the NR3A subunit of the NMDA receptor in recombinant systems and primary cortical neurons. J Neurophysiol. 87:2052-63.

Schäfer, M.K.-H., H. Varoqui, N. Defamie, E. Weihe, and J.D. Erickson. 2002. Molecular cloning and functional identification of mouse vesicular glutamate transporter 3 and its expression in subsets of novel excitatory neurons. J Biol Chem. 277:50734-48.

Schenck, S., S.M. Wojcik, N. Brose, and S. Takamori. 2009. A chloride conductance in VGLUT1 underlies maximal glutamate loading into synaptic vesicles. Nat Neurosci. 12:156-62.

Schrimpf, S.P., V. Meskenaite, E. Brunner, D. Rutishauser, P. Walther, J. Eng, R. Aebersold, and P. Sonderegger. 2005. Proteomic analysis of synaptosomes using isotope-coded affinity tags and mass spectrometry. Proteomics. 5:2531-41.

Searle, B.C., M. Turner, and A.I. Nesvizhskii. 2008. Improving sensitivity by probabilistically combining results from multiple MS/MS search methodologies. J Proteome Res. 7:245-53.

Sheikh, S.N., and D.L. Martin. 1996. Heteromers of glutamate decarboxylase isoforms occur in rat cerebellum. Journal of Neurochemistry. 66:2082-90.

Shepherd, G.M., and K.M. Harris. 1998. Three-dimensional structure and composition of CA3-->CA1 axons in rat hippocampal slices: implications for presynaptic connectivity and compartmentalization. J Neurosci. 18:8300-10.

Shoshan-Barmatz, V., V. De Pinto, M. Zweckstetter, Z. Raviv, N. Keinan, and N. Arbel. 2010. VDAC, a multi-functional mitochondrial protein regulating cell life and death. Mol Aspects Med. 31:227-85.

Singer, P., D. Boison, H. Möhler, J. Feldon, and B.K. Yee. 2009. Deletion of glycine transporter 1 (GlyT1) in forebrain neurons facilitates reversal learning: enhanced cognitive adaptability? Behav Neurosci. 123:1012-27.

Singh, O.V., M. Yaster, J.-T. Xu, Y. Guan, X. Guan, A.M. Dharmarajan, S.N. Raja, P.L. Zeitlin, and Y.X. Tao. 2009. Proteome of synaptosome-associated proteins in spinal cord dorsal horn after peripheral nerve injury. Proteomics. 9:1241-53.

SKOU, J.C. 1957. The influence of some cations on an adenosine triphosphatase from peripheral nerves. Biochim Biophys Acta. 23:394-401.

Smith, A.D. 2006. Prevention of dementia: a role for B vitamins? Nutr Health. 18:225-6.

Smith, L.K., and P.T. Carroll. 1993. Membrane-bound choline-O-acetyltransferase in rat hippocampal tissue is anchored by glycosyl-phosphatidylinositol. Brain Res. 605:155-63.

Sobolevsky, A.I., M.P. Rosconi, and E. Gouaux. 2009. X-ray structure, symmetry and mechanism of an AMPA-subtype glutamate receptor. Nature. 462:745-56.

Söllner, T., S.W. Whiteheart, M. Brunner, H. Erdjument-Bromage, S. Geromanos, P. Tempst, and J.E. Rothman. 1993. SNAP receptors implicated in vesicle targeting and fusion. Nature. 362:318-24.

Song, J.Y., K. Ichtchenko, T.C. Südhof, and N. Brose. 1999. Neuroligin 1 is a postsynaptic celladhesion molecule of excitatory synapses. Proc Natl Acad Sci USA. 96:1100-5.

Staley, J.K., R.T. Malison, and R.B. Innis. 1998. Imaging of the serotonergic system: interactions of neuroanatomical and functional abnormalities of depression. Biol Psychiatry. 44:534-49.

Steiner, J.A., A.M.D. Carneiro, and R.D. Blakely. 2008. Going with the flow: trafficking-dependent and -independent regulation of serotonin transport. Traffic. 9:1393-402.

Sudhof, T.C. 2004. The synaptic vesicle cycle. Annu Rev Neurosci. 27:509-47.

Südhof, T.C. 2008. Neuroligins and neurexins link synaptic function to cognitive disease. Nature. 455:903-11.

Südhof, T.C., and J.E. Rothman. 2009. Membrane fusion: grappling with SNARE and SM proteins. Science. 323:474-7.

Suelter, C.H., and M. DeLuca. 1983. How to prevent losses of protein by adsorption to glass and plastic. Anal Biochem. 135:112-9.

Suh, Y.H., A. Terashima, R.S. Petralia, R.J. Wenthold, J.T.R. Isaac, K.W. Roche, and P.A. Roche. 2010. A neuronal role for SNAP-23 in postsynaptic glutamate receptor trafficking. Nat Neurosci. 13:338-43.

Tabuchi, K., J. Blundell, M.R. Etherton, R.E. Hammer, X. Liu, C.M. Powell, and T.C. Südhof. 2007. A neuroligin-3 mutation implicated in autism increases inhibitory synaptic transmission in mice. Science. 318:71-6. 
Takahashi, S., H. Yamamoto, Z. Matsuda, M. Ogawa, K. Yagyu, T. Taniguchi, T. Miyata, H. Kaba, T. Higuchi, and F. Okutani. 1995. Identification of two highly homologous presynaptic proteins distinctly localized at the dendritic and somatic synapses. FEBS Lett. 368:455-60.

Takamori, S. 2006. VGLUTs: 'exciting' times for glutamatergic research? Neurosci Res. 55:343-51.

Takamori, S., P. Malherbe, C. Broger, and R. Jahn. 2002. Molecular cloning and functional characterization of human vesicular glutamate transporter 3. EMBO Rep. 3:798-803.

Takamori, S., J.S. Rhee, C. Rosenmund, and R. Jahn. 2000. Identification of a vesicular glutamate transporter that defines a glutamatergic phenotype in neurons. Nature. 407:189-94.

Takamori, S., J.S. Rhee, C. Rosenmund, and R. Jahn. 2001. Identification of differentiation-associated brain-specific phosphate transporter as a second vesicular glutamate transporter (VGLUT2). J Neurosci. 21:RC182.

Takayasu, Y., M. lino, Y. Takatsuru, K. Tanaka, and S. Ozawa. 2009. Functions of glutamate transporters in cerebellar Purkinje cell synapses. Acta Physiol (Oxf). 197:1-12.

Tekinay, A.B., Y. Nong, J.M. Miwa, I. Lieberam, I. Ibanez-Tallon, P. Greengard, and N. Heintz. 2009. A role for LYNX2 in anxiety-related behavior. Proc Natl Acad Sci USA. 106:4477-82.

Thomas, C., A. Miller, and G. Westbrook. 2006. Synaptic and Extrasynaptic NMDA Receptor NR2 Subunits in Cultured Hippocampal Neurons. Journal of Neurophysiology. 95:1727.

Thomas, D.D., W.B. Taft, K.M. Kaspar, and G.E. Groblewski. 2001. CRHSP-28 regulates Ca(2+)stimulated secretion in permeabilized acinar cells. J Biol Chem. 276:28866-72.

Thomas, D.D.H., C.L. Martin, N. Weng, J.A. Byrne, and G.E. Groblewski. 2010. Tumor protein D52 expression and Ca2+-dependent phosphorylation modulates lysosomal membrane protein trafficking to the plasma membrane. Am J Physiol, Cell Physiol. 298:C725-39.

Tiacci, E., P.-L. Orvietani, B. Bigerna, A. Pucciarini, G.L. Corthals, V. Pettirossi, M.P. Martelli, A. Liso, R. Benedetti, R. Pacini, N. Bolli, S. Pileri, K. Pulford, M. Gambacorta, A. Carbone, C. Pasquarello, A. Scherl, H. Robertson, M.T. Sciurpi, G. Alunni-Bistocchi, L. Binaglia, J.A. Byrne, and B. Falini. 2005. Tumor protein D52 (TPD52): a novel B-cell/plasma-cell molecule with unique expression pattern and $\mathrm{Ca}(2+)$-dependent association with annexin VI. Blood. 105:2812-20.

Tovar, K.R., and G.L. Westbrook. 1999. The incorporation of NMDA receptors with a distinct subunit composition at nascent hippocampal synapses in vitro. J Neurosci. 19:4180-8.

Towbin, H., T. Staehelin, and J. Gordon. 1979. Electrophoretic transfer of proteins from polyacrylamide gels to nitrocellulose sheets: procedure and some applications. Proc Natl Acad Sci U S A. 76:4350-4.

Traynelis, S.F., L.P. Wollmuth, C.J. McBain, F.S. Menniti, K.M. Vance, K.K. Ogden, K.B. Hansen, H. Yuan, S.J. Myers, R. Dingledine, and D. Sibley. 2010. Glutamate receptor ion channels: structure, regulation, and function. Pharmacol Rev. 62:405-96.

Tribl, F., K. Marcus, G. Bringmann, H.E. Meyer, M. Gerlach, and P. Riederer. 2006. Proteomics of the human brain: sub-proteomes might hold the key to handle brain complexity. Journal of neural transmission (Vienna, Austria : 1996). 113:1041-54.

Tribl, F., H.E. Meyer, and K. Marcus. 2008. Analysis of organelles within the nervous system: impact on brain and organelle functions. Expert review of proteomics. 5:333-51.

Tsien, R.Y. 2009. Constructing and exploiting the fluorescent protein paintbox (Nobel Lecture). Angew Chem Int Ed Engl. 48:5612-26.

Valtorta, F., M. Pennuto, D. Bonanomi, and F. Benfenati. 2004. Synaptophysin: leading actor or walkon role in synaptic vesicle exocytosis? Bioessays. 26:445-53.

van Zundert, B., A. Yoshii, and M. Constantine-Paton. 2004. Receptor compartmentalization and trafficking at glutamate synapses: a developmental proposal. Trends Neurosci. 27:428-37.

Varoqueaux, F., G. Aramuni, R.L. Rawson, R. Mohrmann, M. Missler, K. Gottmann, W. Zhang, T.C. Südhof, and N. Brose. 2006. Neuroligins determine synapse maturation and function. Neuron. 51:741-54.

Varoqueaux, F., S. Jamain, and N. Brose. 2004. Neuroligin 2 is exclusively localized to inhibitory synapses. Eur J Cell Biol. 83:449-56.

Varoqui, H., M.K.H. Schäfer, H. Zhu, E. Weihe, and J.D. Erickson. 2002. Identification of the differentiation-associated $\mathrm{Na}+\mathrm{PI}$ transporter as a novel vesicular glutamate transporter expressed in a distinct set of glutamatergic synapses. J Neurosci. 22:142-55.

Vida, T.A., and S.D. Emr. 1995. A new vital stain for visualizing vacuolar membrane dynamics and endocytosis in yeast. J Cell Biol. 128:779-92.

Vinatier, J., E. Herzog, M.-A. Plamont, S.M. Wojcik, A. Schmidt, N. Brose, L. Daviet, S. El Mestikawy, and B. Giros. 2006. Interaction between the vesicular glutamate transporter type 1 and endophilin A1, a protein essential for endocytosis. J Neurochem. 97:1111-25. 
Voglmaier, S.M., K. Kam, H. Yang, D.L. Fortin, Z. Hua, R.A. Nicoll, and R.H. Edwards. 2006. Distinct endocytic pathways control the rate and extent of synaptic vesicle protein recycling. Neuron. 51:71-84.

Voytas, D. 2000. Agarose Gel Electrophoresis. Current Protocolls in Molecular Biology. 51:2.5A12.5A.9.

Wallén-Mackenzie, A., H. Gezelius, M. Thoby-Brisson, A. Nygård, A. Enjin, F. Fujiyama, G. Fortin, and K. Kullander. 2006. Vesicular glutamate transporter 2 is required for central respiratory rhythm generation but not for locomotor central pattern generation. J Neurosci. 26:12294307.

Walther, D.J., and M. Bader. 2003. A unique central tryptophan hydroxylase isoform. Biochem Pharmacol. 66:1673-80.

Whittaker, V.P. 1959. The isolation and characterization of acetylcholine-containing particles from brain. Biochem J. 72:694-706.

Whittaker, V.P. 1993. Thirty years of synaptosome research. J Neurocytol. 22:735-42.

Witzmann, F.A., R.J. Arnold, F. Bai, P. Hrncirova, M.W. Kimpel, Y.S. Mechref, W.J. McBride, M.V. Novotny, N.M. Pedrick, H.N. Ringham, and J.R. Simon. 2005. A proteomic survey of rat cerebral cortical synaptosomes. Proteomics. 5:2177-201.

Wojcik, S.M., S. Katsurabayashi, I. Guillemin, E. Friauf, C. Rosenmund, N. Brose, and J.-S. Rhee. 2006. A shared vesicular carrier allows synaptic corelease of GABA and glycine. Neuron. 50:575-87.

Wojcik, S.M., J.S. Rhee, E. Herzog, A. Sigler, R. Jahn, S. Takamori, N. Brose, and C. Rosenmund. 2004. An essential role for vesicular glutamate transporter 1 (VGLUT1) in postnatal development and control of quantal size. Proc Natl Acad Sci U S A. 101:7158-63.

Wolf, M.E., J.G. Granneman, and G. Kapatos. 1991a. Characterization of the distribution of G alpha o in rat striatal synaptosomes and its colocalization with tyrosine hydroxylase. Synapse. 9:66-74.

Wolf, M.E., and G. Kapatos. 1989a. Flow cytometric analysis and isolation of permeabilized dopamine nerve terminals from rat striatum. J Neurosci. 9:106-14.

Wolf, M.E., and G. Kapatos. 1989b. Flow cytometric analysis of rat striatal nerve terminals. J Neurosci. 9:94-105.

Wolf, M.E., and G. Kapatos. 1989c. Stimulation of D2 dopamine receptors decreases intracellular calcium levels in rat anterior pituitary cells but not striatal synaptosomes: a flow cytometric study using indo-1. Synapse. 4:353-70.

Wolf, M.E., P.A. LeWitt, M.J. Bannon, L.J. Dragovic, and G. Kapatos. 1991b. Effect of aging on tyrosine hydroxylase protein content and the relative number of dopamine nerve terminals in human caudate. Journal of Neurochemistry. 56:1191-200.

Wolf, M.E., M.J. Zigmond, and G. Kapatos. 1989. Tyrosine hydroxylase content of residual striatal dopamine nerve terminals following 6-hydroxydopamine administration: a flow cytometric study. Journal of Neurochemistry. 53:879-85.

Wu, C.H., L.-S.L. Yeh, H. Huang, L. Arminski, J. Castro-Alvear, Y. Chen, Z. Hu, P. Kourtesis, R.S. Ledley, B.E. Suzek, C.R. Vinayaka, J. Zhang, and W.C. Barker. 2003. The Protein Information Resource. Nucleic Acids Res. 31:345-7.

Yamada, M., H. Saisu, T. Ishizuka, H. Takahashi, and T. Abe. 1999. Immunohistochemical distribution of the two isoforms of synaphin/complexin involved in neurotransmitter release: localization at the distinct central nervous system regions and synaptic types. Neuroscience. 93:7-18.

Yamaguchi, F., K. Yamaguchi, Y. Tai, K. Sugimoto, and M. Tokuda. 2001. Molecular cloning and characterization of a novel phospholemman-like protein from rat hippocampus. Brain Res Mol Brain Res. 86:189-92.

Yee, B.K., E. Balic, P. Singer, C. Schwerdel, T. Grampp, L. Gabernet, I. Knuesel, D. Benke, J. Feldon, H. Mohler, and D. Boison. 2006. Disruption of glycine transporter 1 restricted to forebrain neurons is associated with a procognitive and antipsychotic phenotypic profile. $\mathrm{J}$ Neurosci. 26:3169-81.

Zhu, W., J.W. Smith, and C.-M. Huang. 2010. Mass spectrometry-based label-free quantitative proteomics. J Biomed Biotechnol. 2010:840518. 